\title{
ANALYSIS, DESIGN, AND OPTIMIZATION OF STRUCTURES WITH INTEGRAL COMPLIANT MECHANISMS FOR MID-FREQUENCY RESPONSE
}

\author{
by \\ Ercan M. Dede \\ A dissertation submitted in partial fulfillment \\ of the requirements for the degree of \\ Doctor of Philosophy \\ (Mechanical Engineering) \\ in The University of Michigan \\ 2007
}

Doctoral Committee:

Professor Gregory M. Hulbert, Chair

Professor Noboru Kikuchi

Professor Sridhar Kota

Professor Anthony M. Waas 
"Polish the twofold spirit heart and mind, and sharpen the twofold gaze perception and sight.

When your spirit is not in the least clouded, when the clouds of bewilderment clear away, there is the true void."

- Miyamoto Musashi. The Book of Five Rings. ca. 1645. 
(c) Ercan M. Dede

All rights reserved

2007 
To my family. 


\section{ACKNOWLEDGEMENTS}

I would like to acknowledge the invaluable aid and guidance of my dissertation committee members in the completion of this work. A special thanks is owed to my advisor, Dr. Gregory M. Hulbert, for inspiring many of the creative concepts found throughout this research. Additionally, I would like to thank my colleagues Harish Narayanan and the rest of the Computational Mechanics Laboratory group, Brian Trease, Christine Vehar Jutte, and the remaining members of the Compliant Systems Design Laboratory. I would also like to acknowledge the assistance of Jia Li, Kevin King, Bobby Littrell, and Dr. Matt Castanier in advising me on my prototype testing. Furthermore, Steven Emanuel deserves particular thanks for providing his exceptional manufacturing expertise.

Wholeheartedly, I would like to thank my parents, Bonnie and Metin, and my sister, Suzan, for their lifetime support. My sincere appreciation also extends to all of my long-time friends: John Doris, Aaron Dressner, Steve Kolasa, Robbie Linkner, Todd Linkner, Parag Mody, Don Prior, David Shulman, and Benjamin Tatara. Many thanks are owed to my martial arts instructor, Karl W. Scott III, for his intellectual, physical, and spiritual guidance over the years.

Above all, Lily A. Guzmán deserves my deeply felt gratitude for providing me with tremendous encouragement and continued support. We have grown together over the past several years, and she has brought great happiness to my life. 
TABLE OF CONTENTS

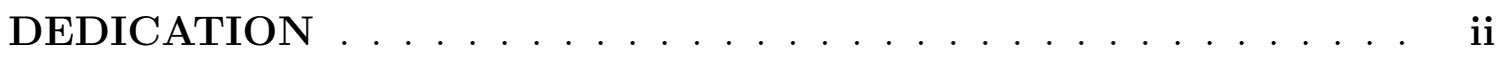

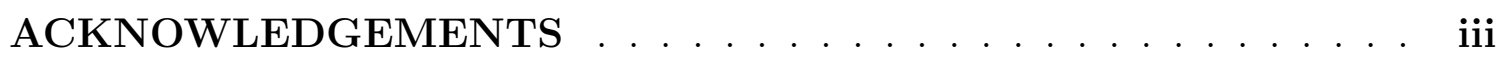

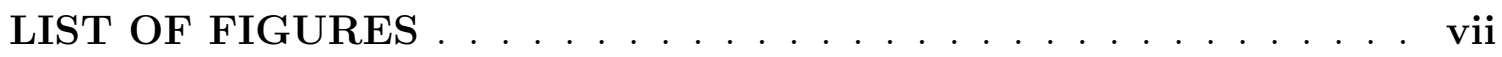

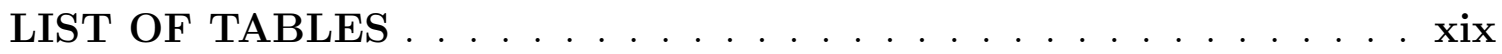

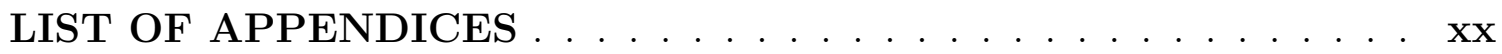

CHAPTER

1. Introduction $\ldots \ldots \ldots \ldots \ldots \ldots \ldots$

1.1 Motivation .................... . . 3

1.2 Background \& Literature Review . . . . . . . . . . . 6

1.2.1 Mid-Frequency Structural Dynamics . . . . . . . . . 7

1.2.2 Analysis of Truss / Lattice Structures . . . . . . . . 9

1.2 .3 Vibration Isolation . . . . . . . . . . . . . . . . . 12

1.2.4 Compliant Mechanism Design . . . . . . . . . . 14

1.2.5 Structural Optimization . . . . . . . . . . 17

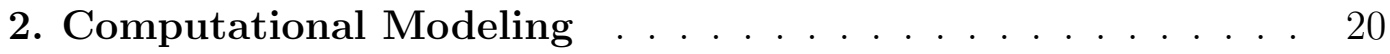

2.1 Conventional Finite Element Formulation: 2-D Rod \& Beam 21

2.2 Spectral Finite Element Formulation: 2-D Rod \& Beam . . . 28

2.3 Spectral Finite Element Formulation: 3-D Space Frame . . . 33

2.4 Verification Model 1: Semi-Infinite Cantilevered Beam . . . . 36

2.5 Verification Model 2: Fixed-Fixed Beam . . . . . . . . . . 40

3. Analysis \& Design . . . . . . . . . . . . . . . . . . 44

3.1 Benchmark Square Core Unit Cell \& Structure . . . . . . . . 45

3.1 .1 Analysis . . . . . . . . . . . . . 4 46

3.1.2 Computational Results . . . . . . . . . . . . . 48

3.2 Compliant Mechanism Unit Cell \& Structure . . . . . . . . 52 
3.2.1 Concept \& Definitions: Compliant Mechanism 1 . . 52

3.2.2 Analysis: Compliant Mechanism 1 . . . . . . . . 58

3.2.3 Computational Results: Compliant Mechanism 1 . . 58

3.2.4 Concept \& Definitions: Compliant Mechanism 2 . . 70

3.2.5 Computational Results: Compliant Mechanism 2 . . 73

3.3 Unit Cell Design using Alternative Materials . . . . . . . . . . 85

3.4 Parallel \& Series Connected Structures . . . . . . . . . . . . . 89

3.4.1 Parallel Connected Structures: Local Forcing . . . . 90

3.4.2 Series Connected Structures: Enhanced Attenuation

Zones ................. 93

3.4.3 Series Connected Structures: Multiple Attenuation

Zones .................. . 99

3.4.4 Series Connected Structures: Introduction to Multi-

Functional Structures . . . . . . . . . . . . . . 102

3.5 3-D Unit Cell \& Structure . . . . . . . . . . . . . . . . . . . 111

3.5.1 Concept \& Definitions . . . . . . . . . . . . . . 111

3.5.2 Analysis ................... . . 113

3.5.3 Computational Results . . . . . . . . . . 115

4. Genetic Algorithm Optimization . . . . . . . . . . . . . . 123

4.1 Size Optimization . . . . . . . . . . . . . . . 124

4.1.1 Analysis .................. . . . 124

4.1 .2 Results . . . . . . . . . . . . . . . 126

4.2 Topology Optimization . . . . . . . . . . . . . . . . . . . . . 129

4.2.1 Analysis . . . . . . . . . . . . . . . . . . . . 129

4.2.2 Results: Example 1 . . . . . . . . . . . . . . 132

4.2.3 Results: Example 2 . . . . . . . . . . . . . . . . 134

4.2.4 Results: Example 3 . . . . . . . . . . . . . 140

4.2 .5 Discussion . . . . . . . . . . . . . . 147

5. Verification \& Validation . . . . . . . . . . . . . . 150

5.1 Prototype Design . . . . . . . . . . . . . . . . . 151

5.1.1 Prototype Concept, Definitions, \& Spectral Element

Analysis . . . . . . . . . . . . . 151

5.1.2 Computational Results: Spectral Elements . . . . . 152

5.2 Prototype Manufacturing . . . . . . . . . . . . . . . 155

5.3 Prototype Testing . . . . . . . . . . . . . . . 156

5.4 Test Results . . . . . . . . . . . . . . . . . . . . . 161

5.5 Experimental Validation . . . . . . . . . . . 163

5.5.1 Analysis: Continuum Elements . . . . . . . . . . . . 164

5.5.2 Computational Results: Continuum Elements . . . . 164

5.6 Discussion ..................... 166

6. Methodology \& Applications . . . . . . . . . . . . . . 171 
6.1 Methodology Overview . . . . . . . . . . . . . . . . 172

6.2 Applications . . . . . . . . . . . . . . . . . . . . . . 174

6.2.1 Acoustic Isolation Panels . . . . . . . . . . . . . . . 174

6.2.2 Vibration Isolation Couplers . . . . . . . . . . . 182

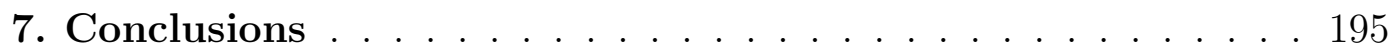

7.1 Future Work . . . . . . . . . . . . . . . . . . . . 195

7.2 Structures having Variable Gain Response . . . . . . . . . . 196

7.2.1 Unit Cell Bottom Layer Stiffness . . . . . . . . . . . 200

7.2 .2 Unit Cell Bottom Layer Mass . . . . . . . . . . . . 203

7.2 .3 Unit Cell Periodicity . . . . . . . . . . . . . . . . . 204

7.2 .4 Internal Actuators . . . . . . . . . . . . . . 208

7.3 Summary . . . . . . . . . . . . . . . . . . . 209

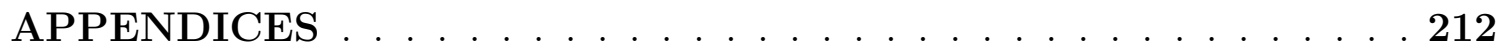

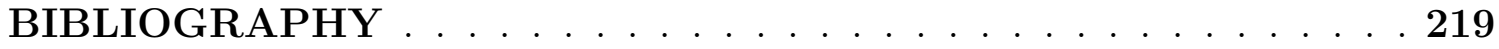




\section{LIST OF FIGURES}

\section{Figure}

1.1 International space station, from NASA [70] . . . . . . . . . 4

1.2 Estimated pressure spectrum for turbulent boundary layer excitation: Boeing model 727-200 aircraft (upper curve for Mach 0.85, lower curve for Mach 0.55), from Bhat \& Wilby [6] . . . . . . . . 5

1.3 Representation of aircraft fuselage structure, from Bhat \& Wilby [6] 6

$1.4 \quad 3-D$ truss made from an aluminum casting alloy, from Wallach \& Gibson $[102] \ldots \ldots \ldots$. . . . . . . . . . . . . . . . . 9

1.5 Cross-section of machine augmented composite, from Hawkins et al. [37] . . . . . . . . . . . . . . . . . . . . . . . . . . 13

1.6 Compliant structure MEM device, from Kota et al. [53] . . . . . . . 15

1.7 Optimized 2-D truss structure, from Keane $[50]$. . . . . . . . . . 18

1.8 Optimized 3-D truss structure, from Moshrefi-Torbati et al. [68] . . 18

2.1 Rod element and beam element nodal degrees of freedom and loads 21

2.2 Rod element and beam element lumped-mass approximation . . . . 23

$2.3 \quad 3-\mathrm{D}$ space frame element nodal degrees of freedom and loads . . . . 34

2.4 Schematic of semi-infinite beam modeled using conventional elements 37

2.5 Schematic of semi-infinite beam modeled using spectral elements . . 37

2.6 Impulse force: (a) time history and (b) FFT . . . . . . . . . . . 38

2.7 Transverse velocity response comparison for semi-infinite beam subject to an impulse load . . . . . . . . . . . . . . . . . . . . . 39 
$2.8 \quad$ Schematic of fixed-fixed beam . . . . . . . . . . . . . . . 41

2.9 Natural frequencies of fixed-fixed beam modeled using two spectral finite elements . . . . . . . . . . . . . . . . . . . . . . . 42

3.1 Square core unit cell (numbers that are circled denote elements, numbers that are not circled denote nodes) $\ldots \ldots \ldots$. . . . . . 46

$3.2 \quad$ Periodic square core structure $\ldots \ldots \ldots \ldots \ldots$

3.3 Top layer RMS velocity of square core structure: (a) unit cell and (b) structure . . . . . . . . . . . . . . . . . 5 50

3.4 Deformed shapes of square core unit cell: (a) $2600 \mathrm{~Hz}$, (b) $2660 \mathrm{~Hz}$, (c) $3700 \mathrm{~Hz}$, and (d) $4600 \mathrm{~Hz} \ldots \ldots \ldots \ldots$. . . . . . . 50

3.5 Deformed shapes of square core structure: (a) $90 \mathrm{~Hz}$, (b) $380 \mathrm{~Hz}$, and (c) $1500 \mathrm{~Hz}$ (dotted lines denote undeformed configuration, continuous lines denote deformed configuration) . . . . . . . . . . 51

3.6 Rigid link 4-bar mechanism diagram (numbers denote links, $(\cdot, \cdot)$ denote instant centers $) \ldots \ldots \ldots \ldots \ldots \ldots$

$3.7 \quad$ Compliant mechanism unit cell model . . . . . . . . . . . . . . 55

$3.8 \quad$ Periodic compliant mechanism structure . . . . . . . . . 55

3.9 Compliant mechanism unit cell model (numbers that are circled denote elements, numbers that are not circled denote nodes) $\ldots . \quad 56$

3.10 Compliant mechanism unit cell FRF . . . . . . . . . . . . . 60

3.11 Top layer RMS velocity comparison for unit cell . . . . . . . . . . 60

3.12 Top layer RMS velocity comparison for structure . . . . . . . . . . 61

3.13 Compliant mechanism unit cell deformed shape and energy distribution at $3500 \mathrm{~Hz}$ (dotted lines denote undeformed configuration, continuous lines denote deformed configuration) . . . . . . . . 63

3.14 Compliant mechanism unit cell deformed shape and energy distribution at $4500 \mathrm{~Hz}$ (dotted lines denote undeformed configuration, continuous lines denote deformed configuration) . . . . . . . . 63 
3.15 Compliant mechanism unit cell deformed shape and energy distribution at $5220 \mathrm{~Hz}$ (dotted lines denote undeformed configuration, continuous lines denote deformed configuration) . . . . . . . . . . . 64

3.16 Compliant mechanism unit cell deformed shape and energy distribution at $\sim 6000 \mathrm{~Hz}$ (dotted lines denote undeformed configuration, continuous lines denote deformed configuration) . . . . . . . . . . 64

3.17 Square core structure SPL at $1780 \mathrm{~Hz}$ - units: dB re 20E-6 Pa . . . 65

3.18 Compliant mechanism core structure SPL at $1780 \mathrm{~Hz}-$ units: dB re 20E-6 $\mathrm{Pa} \ldots \ldots \ldots . \ldots . \ldots 65$

3.19 Square core structure SPL at $3870 \mathrm{~Hz}$ - units: dB re 20E-6 Pa . . . 66

3.20 Compliant mechanism core structure SPL at $3870 \mathrm{~Hz}-$ units: dB re 20E-6 $\mathrm{Pa} \ldots \ldots \ldots 66$

3.21 Convergence of far-field SPL at $1700 \mathrm{~Hz}$ : (a) $m=10$, (b) $m=100$, and (c) $m=200$ - units: dB re 20E-6 Pa . . . . . . . . . . 68

3.22 Percent difference between far-field SPL distribution at $1700 \mathrm{~Hz}$ : (a) $m=100$ and $m=10$; (b) $m=200$ and $m=100 \ldots 68$

3.23 Spatial convergence of far-field SPL distribution at $3870 \mathrm{~Hz}$ : (a) 0.0125 m resolution; (b) 0.00625 m resolution - units: dB re 20E-6 Pa 69

3.24 Deformed shapes of compliant mechanism structure: (a) $40 \mathrm{~Hz}$, (b) $150 \mathrm{~Hz}$, and (c) $3000 \mathrm{~Hz}$ (dotted lines denote undeformed configuration, continuous lines denote deformed configuration) . . . . . . . . 69

3.25 Rigid link diagram of one half of rhombus mechanism (numbers de-

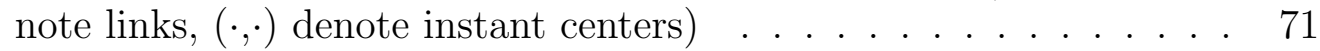

3.26 Rhombus compliant mechanism unit cell model . . . . . . . . . 71

3.27 Rhombus compliant mechanism unit cell model (numbers that are circled denote elements, numbers that are not circled denote nodes) $\quad 72$

3.28 Rhombus compliant mechanism unit cell FRF . . . . . . . . . . 73

3.29 Rhombus compliant mechanism unit cell deformed shape and energy distribution at $3000 \mathrm{~Hz}$ (dotted lines denote undeformed configuration, continuous lines denote deformed configuration) . . . . . . . . 75 
3.30 Rhombus compliant mechanism unit cell deformed shape and energy distribution at $4000 \mathrm{~Hz}$ (dotted lines denote undeformed configuration, continuous lines denote deformed configuration) . . . . . . . . 76

3.31 Rhombus compliant mechanism unit cell deformed shape and energy distribution at $5110 \mathrm{~Hz}$ (dotted lines denote undeformed configuration, continuous lines denote deformed configuration) . . . . . . . 76

3.32 Rhombus compliant mechanism unit cell deformed shape and energy distribution at $6620 \mathrm{~Hz}$ (dotted lines denote undeformed configuration, continuous lines denote deformed configuration) . . . . . . . 77

3.33 Rhombus compliant mechanism unit cell deformed shape and energy distribution at $7420 \mathrm{~Hz}$ (dotted lines denote undeformed configuration, continuous lines denote deformed configuration) . . . . . . . 77

3.34 Rhombus compliant mechanism unit cell deformed shape and energy distribution at $9240 \mathrm{~Hz}$ (dotted lines denote undeformed configuration, continuous lines denote deformed configuration) . . . . . . . 78

3.35 Rhombus compliant mechanism unit cell deformed shape and energy distribution at $9600 \mathrm{~Hz}$ (dotted lines denote undeformed configuration, continuous lines denote deformed configuration) . . . . . . . 78

3.36 Rhombus compliant mechanism unit cells with varying mechanical advantage: (a) $\mathrm{MA}=7$, (b) $\mathrm{MA}=5$, (c) $\mathrm{MA}=3$, (d) $\mathrm{MA}=2$, and (e) $\mathrm{MA}=1 \ldots \ldots \ldots \ldots$. . . . . . . . . . . . . . . . . 79

3.37 Top layer RMS velocity comparison for rhombus compliant mechanism unit cells with varying mechanical advantage . . . . . . . . . 80

3.38 Rhombus compliant mechanism $(\mathrm{MA}=7)$ unit cell FRF . . . . . 81

3.39 Rhombus compliant mechanism $(\mathrm{MA}=5)$ unit cell FRF . . . . . 81

3.40 Rhombus compliant mechanism $(\mathrm{MA}=3)$ unit cell FRF . . . . . . 82

3.41 Rhombus compliant mechanism $(\mathrm{MA}=2)$ unit cell FRF . . . . . 82

3.42 Top layer RMS velocity for rhombus compliant mechanism unit cell having $\mathrm{MA}=1 \ldots \ldots . \ldots . \ldots . \ldots . \ldots 83$ 
3.43 Rhombus compliant mechanism unit cell deformed shape and energy distribution at the first attenuation zone cutoff frequency (dotted lines denote undeformed configuration, continuous lines denote deformed configuration, line thickness denotes energy content): (a) $\mathrm{MA}=7$ at $5120 \mathrm{~Hz}$, (b) $\mathrm{MA}=5$ at $5550 \mathrm{~Hz}$, (c) MA = 3 at 5680 $\mathrm{Hz},(\mathrm{d}) \mathrm{MA}=2$ at $5870 \mathrm{~Hz}$, and (e) $\mathrm{MA}=1$ at $4160 \ldots . . . .884$

3.44 Elastic (i.e. storage) modulus, viscous (i.e. loss) modulus, and loss tangent of ABS as a function of temperature, from Sepe [89] . . . . 87

3.45 Schematic representation of relative unit cell sizing resulting from material selection (not shown to actual scale) . . . . . . . . . . . . 88

3.46 ABS compliant mechanism unit cell FRF . . . . . . . . . . . 89

3.47 Homogeneous periodic square core structure subjected to a localized distributed load . . . . . . . . . . . . . . . . . . 91

3.48 Heterogeneous structure comprised of square core unit cells connected in parallel with compliant mechanism units cells and subjected to a localized distributed load . . . . . . . . . . . . . . 91

3.49 Top layer RMS velocity of square core structure subjected to localized forcing versus a structure comprised of square core unit cells connected in parallel with compliant mechanism units cells and subjected to the same localized forcing . . . . . . . . . . . . .

3.50 Rhombus compliant mechanism unit cell model (the number denotes the top layer middle node) . . . . . . . . . . . . . . . . . . . 94

3.51 Rhombus compliant mechanism unit cell series structures (structures increase in the number of layers from (a) one unit cell to (t) 20 unit cells in series $)$. . . . . . . . . . . . . . . . . . . . . . 95

3.52 Top layer RMS velocity of rhombus compliant mechanism series structures . . . . . . . . . . . . . . . . 96

3.53 Top layer RMS velocity of rhombus compliant mechanism series structures from $0 \mathrm{~Hz}$ to $1500 \mathrm{~Hz}$. . . . . . . . . . . . . . 96

3.54 Top layer transverse and horizontal RMS velocity of rhombus compliant mechanism series structures . . . . . . . . . . . . . . 97 
3.55 Energy distribution in the top three rhombus compliant mechanism unit cells for the structure with 20 unit cells in series: (a) 4410 $\mathrm{Hz}$, (b) $4440 \mathrm{~Hz}$, and (c) $4480 \mathrm{~Hz}$ (dotted lines denote undeformed configuration, continuous lines denote deformed configuration) . . .

3.56 Two unit cells and their assembly into a series structure: (a) bottom unit cell, (b) top unit cell, and (c) series compliant mechanism structure (thicker lines denote members with a width of $2.0 \mathrm{~mm}$, thinner lines denote members with a width of $1.0 \mathrm{~mm})$. . . . . . . . . 100

3.57 Top layer RMS velocity of bottom unit cell, top unit cell, and series compliant mechanism structure . . . . . . . . . . . . . . . . 101

3.58 Two individual unit cells and their assembly into a series structure: (a) bottom unit cell, (b) top unit cell, and (c) double layer square core unit cell (thicker lines denote members with a width of $5.0 \mathrm{~mm}$, thinner lines denote members with a width of $2.5 \mathrm{~mm}$ ) . . . . . . . 104

3.59 Periodic structure comprising six double layer square core unit cells 104

3.60 Two individual unit cells and their assembly into a series structure: (a) bottom unit cell, (b) top unit cell, and (c) multi-functional unit cell (thicker lines denote members with a width of $5.0 \mathrm{~mm}$, thinner lines denote members with a width of $2.5 \mathrm{~mm}$ ) . . . . . . . . 105

3.61 Periodic structure comprising six multi-functional unit cells . . . . . 105

3.62 Top layer RMS velocity of bottom unit cell and double layer square core unit cell . . . . . . . . . . . . . . . . . . . . . 106

3.63 Top layer RMS velocity of double layer square core unit cell and structure . . . . . . . . . . . . . . . . . 107

3.64 Top layer RMS velocity of bottom unit cell, top unit cell, and multifunctional unit cell . . . . . . . . . . . . . . . . . . 109

3.65 Top layer RMS velocity of multi-functional unit cell and structure . 109

3.66 Top layer RMS velocity of double layer square core unit cell versus multi-functional unit cell . . . . . . . . . . . . . . . . . . . 110

3.67 Top layer RMS velocity of double layer square core structure versus multi-functional structure . . . . . . . . . . . . . . . . 110

3.68 3-D rhombus compliant mechanism unit cell model (numbers denote

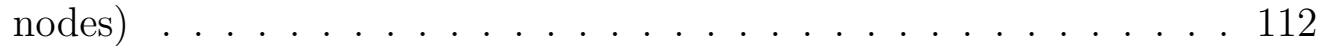


$3.693-\mathrm{D}$ periodic rhombus compliant mechanism lattice panel structure . 112

$3.703-\mathrm{D}$ plane wave incident to the bottom layer of the structure . . . . 114

3.713 -D rhombus compliant mechanism unit cell FRF . . . . . . . . 116

3.72 Top layer RMS velocity of 3-D lattice panel structure versus unit cell 117

3.73 Three-dimensional rhombus compliant mechanism unit cell deformed shape and energy distribution at: (a) $2500 \mathrm{~Hz}$, (b) $5310 \mathrm{~Hz}$, and (c) $9000 \mathrm{~Hz}$ (dotted lines denote undeformed configuration, continuous lines denote deformed configuration) . . . . . . . . . . . . . 119

$3.743-\mathrm{D}$ rhombus compliant mechanism lattice panel structure energy distribution - viewed edge on for visual clarity - at the following frequencies: (a) $2650 \mathrm{~Hz}$, (b) $2780 \mathrm{~Hz}$, (c) $3000 \mathrm{~Hz}$, (d) $3190 \mathrm{~Hz}$, (e) $3430 \mathrm{~Hz}$, (f) $3540 \mathrm{~Hz}$, (g) $3840 \mathrm{~Hz}$, and (h) $4490 \mathrm{~Hz}$. . . . . . . . 120

3.753 -D rhombus compliant mechanism lattice panel structure deformed shape and energy distribution at the following frequencies: (a) 2650 $\mathrm{Hz}$ and (b) $8040 \mathrm{~Hz}$ (dotted lines denote undeformed configuration, continuous lines denote deformed configuration) . . . . . . . . . 122

4.1 Compliant mechanism unit cell model (numbers that are circled denote elements) . . . . . . . . . . . . . . 125

4.2 Flowchart of computations for genetic algorithm size optimization . 126

4.3 Top layer RMS velocity of compliant mechanism unit cell . . . . . . 127

4.4 Initial compliant mechanism unit cell . . . . . . . . . . . . 128

4.5 Optimized compliant mechanism unit cell . . . . . . . . . . 128

4.6 Initial (pre-optimization) unit cell binary ground structure . . . . . 130

4.7 Flowchart of computations for genetic algorithm topology optimization131

4.8 Initial sizing of unit cell binary ground structure (thickest lines have a width of $5.0 \mathrm{~mm}$; intermediate thickness lines have a width of 2.5 $\mathrm{mm}$; thinnest lines have a width of $0.5 \mathrm{~mm}$ ) . . . . . . . . . 133

4.9 Top ten unit cell topologies from optimization run 1 (140 members iterated over 10 generations $)$. . . . . . . . . . . 135 
4.10 Top layer RMS velocity of top ten unit cell topologies from optimization run 1 (dashed lines denote initial response, continuous lines denote filtered response) . . . . . . . . . . . . . . 136

4.11 Average FV of 400 member population over 400 generations . . . . 137

4.12 Top ten unit cell topologies from optimization run 2 (400 members

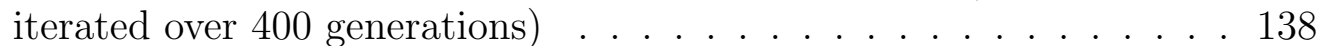

4.13 Top layer RMS velocity of top ten unit cell topologies from optimization run 2 (dashed lines denote initial response, continuous lines denote filtered response) . . . . . . . . . . . . . . . 139

4.14 Third best unit cell topology from optimization run 3 (140 members

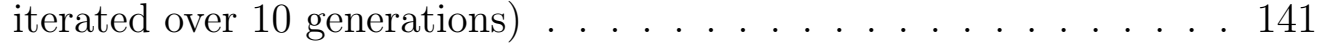

4.15 Reduced unit cell topology (thickest lines have a width of $5.0 \mathrm{~mm}$; intermediate thickness lines have a width of $2.5 \mathrm{~mm}$; thinnest lines have a width of $0.6 \mathrm{~mm})$. . . . . . . . . . . . . . . . 141

4.16 Top layer RMS velocity response of initial, filtered, and reduced unit

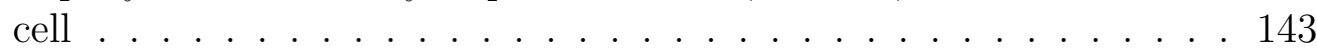

4.17 Reduced unit cell model (numbers denote nodes) . . . . . . . . . . 143

4.18 FRF of reduced unit cell topology . . . . . . . . . . . . . . . . 144

4.19 Reduced compliant mechanism unit cell deformed shape and energy distribution at $4000 \mathrm{~Hz}$ (dotted lines denote undeformed configuration, continuous lines denote deformed configuration) . . . . . . . . 145

4.20 Reduced compliant mechanism unit cell deformed shape and energy distribution at $6550 \mathrm{~Hz}$ (dotted lines denote undeformed configuration, continuous lines denote deformed configuration) . . . . . . . 145

4.21 Reduced compliant mechanism unit cell deformed shape and energy distribution at $9240 \mathrm{~Hz}$ (dotted lines denote undeformed configuration, continuous lines denote deformed configuration) . . . . . . . 146

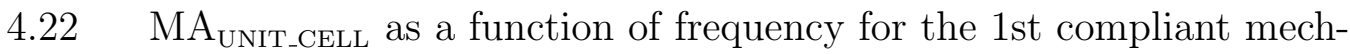
anism unit cell (Section 3.2.1), the 2nd (i.e. rhombus) compliant mechanism unit cell (Section 3.2.4), and the reduced unit cell . . . . 148

5.1 Rhombus compliant mechanism unit cell model . . . . . . . . . 151

5.2 Periodic rhombus compliant mechanism structure . . . . . . . . . 152 
5.3 Rhombus compliant mechanism unit cell FRF . . . . . . . . . 154

5.4 Top layer RMS velocity (computed using the response from 23 top layer nodes) for compliant mechanism structure . . . . . . . . 155

5.5 Solid model geometry of rhombus compliant mechanism structure . 156

5.6 Machining of compliant mechanism structure on 3-axis CNC mill . . 157

5.7 Machining of pocket geometry for compliant mechanism structure using 1/8-inch diameter end mill: (a) pocket geometry and (b) machine fixturing . . . . . . . . . . . . . . 157

$5.8 \quad$ Experimental test setup for compliant mechanism structure . . . . 158

5.9 Experimental test setup for square core (test control) structure . . 158

5.10 Experimental measurement system: (a) single point laser vibrometer (SPLV) and (b) oscilloscope . . . . . . . . . . . . . 159

5.11 Speaker calibration SPL spectrum at $1 \mathrm{~m}$ scaled to $0.64 \mathrm{~mm} \ldots 161$

5.12 Spectral finite element vs. experimental RMS transverse velocity (computed using the response from 2 top layer nodes) for square core structure . . . . . . . . . . . . . . . . . 162

5.13 Spectral finite element vs. experimental RMS transverse velocity (computed using the response from 2 top layer nodes) for compliant mechanism structure . . . . . . . . . . . . . . . . 162

5.14 Nastran vs. experimental RMS transverse velocity (computed using the response from 2 top layer nodes) for square core structure $\ldots 165$

5.15 Nastran vs. experimental RMS transverse velocity (computed using the response from 2 top layer nodes) for compliant mechanism structure 165

5.16 Global mode of compliant mechanism structure $-3963 \mathrm{~Hz} \quad \ldots . .166$

5.17 Percent error between Nastran and experimental RMS transverse velocity results for square core structure . . . . . . . . . 167

5.18 Percent error between Nastran and experimental RMS transverse velocity results for compliant mechanism structure . . . . . . . 167

5.19 Symmetric mode of compliant mechanism structure $-8739 \mathrm{~Hz}$. . 169 
5.20 Anti-symmetric mode of compliant mechanism structure $-8797 \mathrm{~Hz}$

5.21 Nastran vs. experimental RMS transverse velocity (computed using the response from 2 top layer nodes) for compliant mechanism structure. Region of interest is highlighted by the dashed oval. . . . 169

6.1 Estimated pressure spectrum for turbulent boundary layer excitation: Boeing model 727-200 aircraft (upper curve for Mach 0.85, lower curve for Mach 0.55), from Bhat \& Wilby [6] . . . . . . . . 175

6.2 Representation of aircraft fuselage structure, from Bhat \& Wilby [6] 175

6.3 Conceptual model of fuselage radiating element approximated by a two-dimensional beam . . . . . . . . . . . . . 176

6.4 Conceptual model of fuselage radiating element approximated by a two-dimensional truss-like structure with integral compliant mechanisms . . . . . . . . . . . . . . . . . . . 176

6.5 Estimated pressure spectrum for turbulent boundary layer excitation: (a) Boeing model 727-200 aircraft (upper curve for Mach 0.85, lower curve for Mach 0.55), from Bhat \& Wilby [6]; (b) reconstructed data . . . . . . . . . . . . . . . . . . . . 179

6.6 Top layer RMS velocity of bare fuselage structure, compliant mechanism structure, and compliant mechanism unit cell . . . . . . . . 180

6.7 Conceptual model of fuselage structure with integral compliant mechanisms . . . . . . . . . . . . . . . . . . . . . . 181

6.8 Conceptual model of vibration isolator coupling with an integral annular compliant mechanism structure . . . . . . . . . . . . . 184

6.9 Top layer RMS velocity of multi-material rhombus compliant mechanism unit cell . . . . . . . . . . . . . . . . . . 185

6.10 Finite element solid model of one quarter of the annular compliant mechanism structure (purple elements represent vulcanized rubber; green elements represent spring steel) $\ldots \ldots$. . . . . . . . 186

6.11 Boundary conditions for the finite element solid model of one quarter of the annular compliant mechanism structure . . . . . . . . 187 
6.12 Finite element solid model of one quarter of the annular compliant mechanism structure: (a) bottom layer loading and (b) nodal locations for top layer response . . . . . . . . . . . . . 188

6.13 Top layer normal velocity of the annular compliant mechanism structure quarter model at the monitored nodal locations . . . . . . . . . 190

6.14 Top layer RMS velocity of the annular compliant mechanism structure quarter model . . . . . . . . . . . . . . . . . 190

6.15 Displacement contours superimposed on the normal mode shapes of one quarter of the annular compliant mechanism structure: (a) 201 Hz, (b) $293 \mathrm{~Hz}$, (c) $396 \mathrm{~Hz}$, (d) $482 \mathrm{~Hz}$, (e) $699 \mathrm{~Hz}$, and (f) $990 \mathrm{~Hz}$. 192

6.16 Displacement contours superimposed on the normal mode shapes of the compliant mechanism core of one quarter of the annular compliant mechanism structure: (a) $396 \mathrm{~Hz}$ and (d) $482 \mathrm{~Hz}$. . . . . . . . 193

7.1 Normalized frequency as a function of a fraction of the normalized unit cell bottom layer stiffness, bottom layer mass, and periodicity . 199

7.2 Relationship between unit cell response and increased bottom layer stiffness . . . . . . . . . . . . . . . . . . 201

7.3 Conceptual model of a compliant mechanism unit cell having a composite bottom layer designed for variable gain response . . . . . . . 202

7.4 MR material behavior and composite beam structural dynamic response: (a) shear stress-shear strain relationship for MR materials, and (b) transverse vibration response for MR composite beam, from Yalcintas \& Dai [105] . . . . . . . . . . . . . . . . 202

7.5 Relationship between unit cell response and decreased bottom layer mass . . . . . . . . . . . . . . . . . . . 204

7.6 Graphical representation of the modification of unit cell periodicity 205

7.7 Relationship between unit cell response and decreased unit cell length (i.e. periodicity) . . . . . . . . . . . . . . 205

7.8 Cellular topologies enabled using new NiTi brazing method, from Grummon, Shaw, \& Foltz [32] . . . . . . . . . . . . . . 207

7.9 Mechanical response of a niobium-brazed open cell honeycomb structure built from NiTi strip material, from Grummon, Shaw, \& Foltz [32] 207 
7.10 Rhombus compliant mechanism unit cells with varying mechanical advantage: (a) $\mathrm{MA}=7$, (b) $\mathrm{MA}=5$, and (c) $\mathrm{MA}=1$ (asterisks denote locations of internal actuators) . . . . . . . . . . . 208

7.11 Top layer RMS velocity comparison for rhombus compliant mechanism unit cells with varying mechanical advantage due to internal shape change via actuators . . . . . . . . . . . . . 208

B.1 Schematic of acoustic excitation system . . . . . . . . . . 216

B.2 Assembled high pass filter (including dummy load) . . . . . . . 218 


\section{LIST OF TABLES}

\section{$\underline{\text { Table }}$}

$2.1 \quad$ Semi-infinite beam physical parameters . . . . . . . . . . . . 37

$2.2 \quad$ Fixed-fixed beam physical parameters . . . . . . . . . . . . . . 41

$2.3 \quad$ Natural frequencies of fixed-fixed beam . . . . . . . . . . . . . 43

4.1 Initial and optimized sizing of the compliant mechanism unit cell . . 128

B.1 Measurement ranges for velocity decoder OFV-2602, from Polytec [81]218 


\section{LIST OF APPENDICES}

\section{Appendix}

A. Stiffness Matrix Components for Spectral Beam Element _. . . . . 213

B. Acoustic Excitation \& SPLV Measurement System . . . . . . . . . 215

B.1 Acoustic Excitation System . . . . . . . . . . . . . . 215

B.2 SPLV Measurement System . . . . . . . . . . . . 218 


\section{CHAPTER 1}

\section{Introduction}

An analysis, design, and optimization methodology for structures that vibrate in the $1 \mathrm{kHz}$ to $10 \mathrm{kHz}$ frequency range has been developed. This methodology is the synthesis of several established research fields including structural dynamics, compliant mechanism design, finite element computational analysis, and structural optimization via an evolutionary algorithm.

Three primary contributions are outlined in this dissertation, the first of which is the unification of the computational methods required for analyzing and designing structures with integral compliant mechanisms. In Chapter 2 a review of the underlying finite element theory is given, and this review is followed by two specific numerical examples for method verification.

The next two chapters represent the second primary contribution of this work. The analysis and design of structures with integral compliant mechanisms is introduced in Chapter 3. Structural size and topology optimization using a genetic algorithm is then discussed in Chapter 4. Multiple spatial scales are examined including smaller "unit cell" structures that are integrated into larger "global" structures in order to attenuate vibro-acoustic response. Extensions to this methodology are discussed including: unit cell designs using alternative (i.e. nonmetallic) materials; parallel and serial connected structures for enhanced attenuation or multi-functional properties; and the analysis and design of three-dimensional (3-D) structures. Supplementing 
this work, size and topology optimization are brought forth to "tune" structural dynamic response to a desired frequency range, and to illuminate possible alternative unit cell topologies, respectively. Hence, the goal in these chapters is to establish the concepts, definitions, and analytical tools associated with designing structures with integral compliant mechanisms, and to illustrate the design process with numerical examples.

The third primary contribution of this research is set forth in Chapter 5 where the analysis, design, manufacturing, and vibro-acoustic testing of two prototype structures is explained. The first structure investigated has a traditional square core design prevalent throughout much of the prior literature, thus serving as a control for comparison. The second structure has a compliant mechanism core. In this chapter the experimentally measured dynamic response of each structure is quantitatively evaluated and compared with computational predictions.

In Chapter 6 an overview of the analysis, design, and optimization methodology is provided, synthesizing the contributions listed above. Two potential applications of this research are discussed including the manufacturing of practical vibro-acoustic isolation panels for aerospace vehicles, and the design of vibration isolation couplers for rotating shafts in automotive vehicles.

Chapter 7 concludes this dissertation with a discussion of future directions for this research. These directions include the development of adaptive-passive, active, and multi-material/fluid-filled structures, in addition to the development of possible biological sensors and structures. Lastly, the major accomplishments of this research are summarized. 


\subsection{Motivation}

Hearing loss prevention has been declared one of the 21 priority areas for research in the next century according to The National Institute for Occupational Safety and Health (NIOSH) [72]. It has been reported by NIOSH that roughly 30 million Americans are exposed to noise induced hearing loss (NIHL) hazards in their work environment. Furthermore, Kosko [52] reports that studies have shown that noise can cause stress that results in cases of long-term memory impairment, loss of ability to read, and sleep deprivation. Looking to the source of noise in our world, we see that engineered structures are seldom subjected to purely static environments since we live in a dynamic world where objects are in motion. This dynamic environment generates mechanical vibrations that at increased frequencies create noise, which not only has an adverse effect on human performance, but also diminishes perceived product quality as discussed by Frampton \& Clark [26].

Traditional analysis of vibrations has focused on lower frequency response since these modes produce the largest displacement. More recently, the middle to high frequency region has gained attention in conjunction with fatigue, sound transmission, and lightweight vehicle response. Spring-mass absorbers, visco-elastic coatings, and active control solutions have proven effective in vibration attenuation across these frequencies. Nonetheless, these solutions can lead to other problems like increased system complexity or weight since they are commonly an addition once a problem has been identified. Moreover, modal analysis in the mid-frequency range presents a challenge due to the lack of a straightforward computational strategy. Thus, the motivation behind this research is to develop a comprehensive methodology that couples an efficient computational approach to a novel design paradigm for the reduction of 


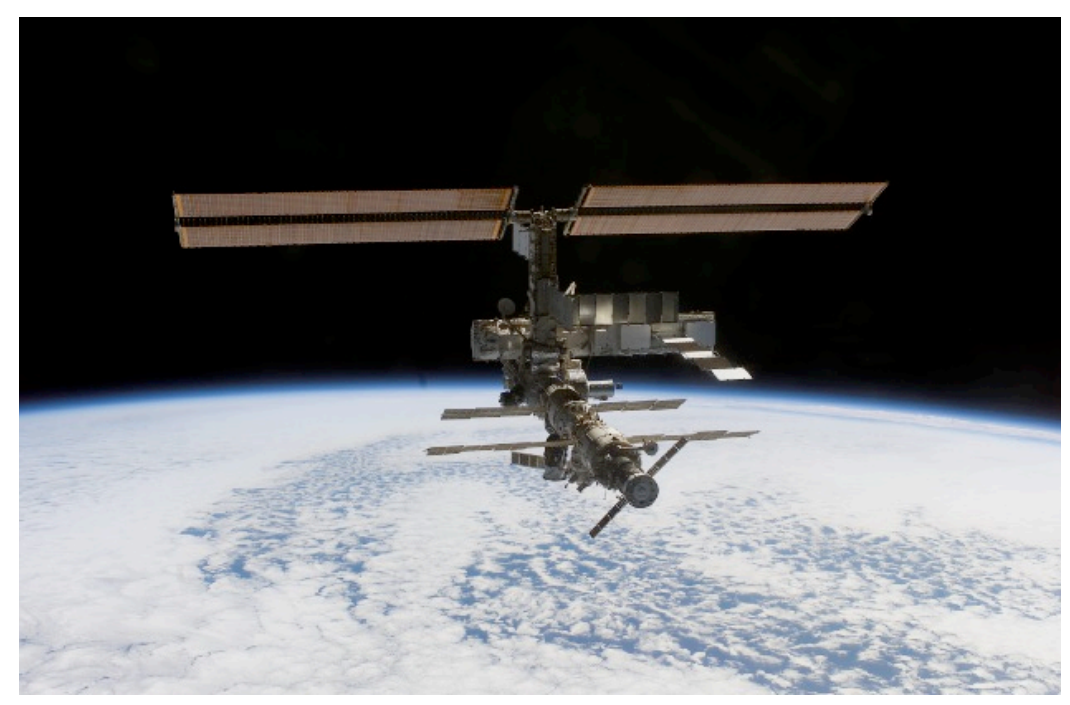

Figure 1.1: International space station, from NASA [70]

mid-frequency structural-borne noise.

Fields of application for this research are accordingly broad and include mechanical, aerospace, naval, and civil engineering, or any application in which structural response generates or transmits noise. One example of a current need for such a methodology can be found on the International Space Station (ISS) shown in Figure 1.1. The American Association for the Advancement of Science [1] and Oberg [74] have reported that many of the valves, fans, and pumps that are mounted to the ISS excite resonant modes within the structure. As a result, a considerable amount of structural-borne noise is generated upwards of $70 \mathrm{~dB}$ within the workspace for the astronauts. The Military Audiology Association [63] has studied this problem in depth since there is a concern that astronauts are not able to obtain "auditory rest." In fact, long-term space flight has been shown to produce 30 to $50 \mathrm{~dB}$ of NIHL due to exposure to noise in the $4 \mathrm{kHz}$ to $6 \mathrm{kHz}$ range. Absorbent padding has served as the "band-aid" solution thus far, but for future space flight a more robust approach that is an integral part of the design process is appropriate.

Further research associated with the phenomenon of turbulent wall pressure fluc- 


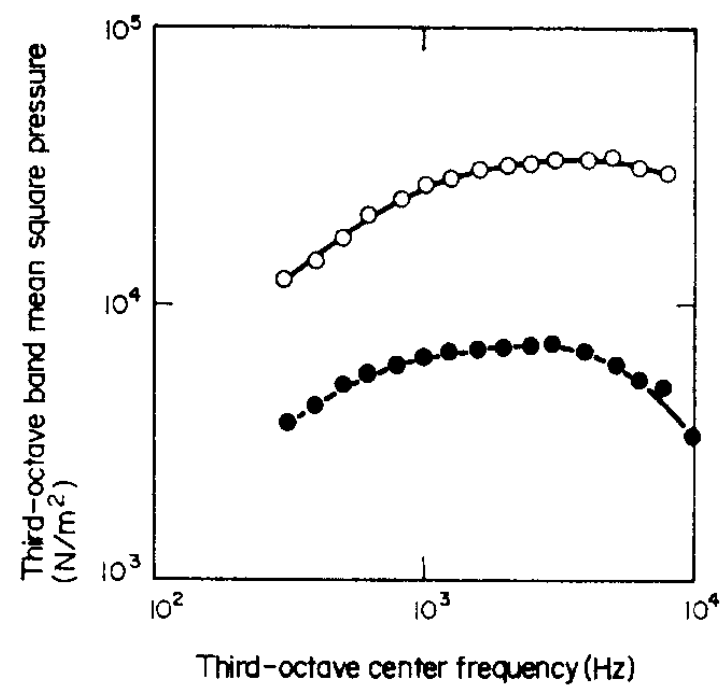

Figure 1.2: Estimated pressure spectrum for turbulent boundary layer excitation: Boeing model 727-200 aircraft (upper curve for Mach 0.85, lower curve for Mach 0.55), from Bhat \& Wilby [6]

tuations has also provided various potential applications for this research as outlined in the comprehensive review by Bull [12]. It is known that surface-pressure fluctuations give rise to several engineering problems: 1) the vibrational generation of acoustic radiation into space, such as a vehicle cabin; 2) the generation of external acoustic radiation from piping systems by vibration of the pipe walls excited through turbulent flow; 3) the transmission of pressure fluctuations through jackets of towed underwater sonar detection arrays; and 4) the generation of acoustic radiation into the flow as a result of vibration of a flexible boundary surface excited by a fluctuating pressure field.

Specific to item 1, commercial aircraft design has revealed that a common source of noise in aircraft cabins is turbulent boundary layer flow causing pressure fluctuations on, and excitation of, the fuselage exterior. Bhat \& Wilby $[5,6]$ note that the frequency spectrum of excitation at common cruise speeds generally extends upwards of $10 \mathrm{kHz}$ as shown in Figure 1.2. Traditional approaches to this problem include 


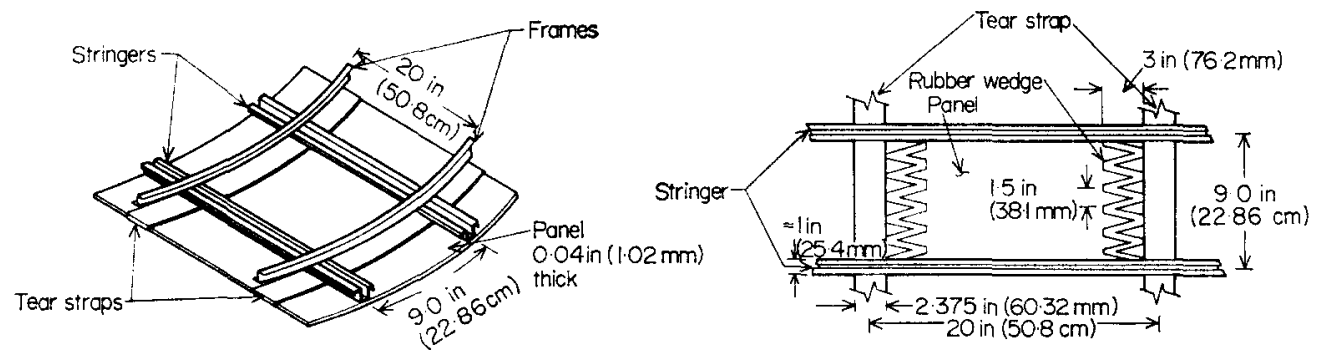

Figure 1.3: Representation of aircraft fuselage structure, from Bhat \& Wilby [6]

the application of damping tape or rubber wedges to the inside skin of the aircraft fuselage to reduce vibro-acoustic response as illustrated in Figure 1.3. More recent approaches include active control to either cancel structural vibrations directly, or to cancel acoustic pressure fluctuations within the cabin, per Gardonio [29].

While these solutions are effective for this specific engineering problem, a more comprehensive approach to the design of structures that minimizes overall system complexity is warranted. Specifically, the research presented in this dissertation is aimed at demonstrating that addressing vibro-acoustic noise challenges during the design stage of a structure can lead to a reduction in transmitted noise, thus allowing for the structure to fulfill multi-functional roles. In other words, the structure not only meets strength requirements, but also facilitates a quieter operating environment without the addition of complicated electronics or excessive weight. This translates into increased customer satisfaction and reduced acoustic signatures, both of which are desirable from an engineering standpoint.

\subsection{Background \& Literature Review}

With regard to engineered structures, the broad topics of computational mechanics and dynamics, compliant mechanism design, and structural optimization are synthesized in this research. From these broad topics there are five specific areas 
of research that are drawn upon to assess the current state of the arts: computational methods for mid-frequency structural dynamic response, truss/lattice vibration analysis, passive versus active vibration isolation, static and dynamic analysis of compliant mechanisms, and structural optimization of trusses using evolutionary algorithms. The subsequent sections provide a literature review of the pertinent research within each of these fields.

\subsubsection{Mid-Frequency Structural Dynamics}

A thorough understanding of existing computational approaches is a prerequisite to the development of a rigorous methodology for the design of structures in the midfrequency range. Since any computational analysis is only as good as its accuracy, a robust analytical strategy must be formed. Furthermore, a complete understanding

of the fundamental dynamics at hand must be sought prior to the introduction of such an approach. To this end, the dynamic analysis of mechanical systems can be divided in to three general frequency regimes including low, middle, and high as outlined by Vlahopoulos \& Zhao [100].

In the low-frequency regime all members of a structure are short relative to the wavelength of vibration. Conventional finite element analysis (FEA) has been shown to be an acceptable computational technique in such applications since modal density is low and natural frequency uncertainty is relatively small, as discussed by Huebner \& Thornton [41], Shabana [90], and Yang [106].

In the high-frequency regime all members of a structure are long relative to the wavelength of vibration. Conventional FEA is incapable of capturing higher order modes and wave phenomena within the system unless the number of elements is increased prohibitively. To reduce computational time and uncertainty, statistical energy analysis (SEA) and energy finite element analysis (EFEA) methods were de- 
veloped by Lyon [62] and Bouthier \& Bernhard [10], respectively. These methods allow for proper characterization of structural dynamics for high-frequency applications and they work well when all members of the structure have high modal density.

The mid-frequency regime is then considered when some members of the structure are long and the rest are short relative to the wavelength of vibration. In this case, FEA is still unreasonable in terms of computational cost due to discretization of long members, while both SEA and EFEA contain assumptions that are relevant only when every component of a structure has high modal density. Due to the short members of the structure, these energy methods cannot properly predict resonant effects. Accordingly, hybrid finite element methods merging FEA and EFEA have been proposed by Vlahopoulos \& Zhao [100]. While this computational strategy produces acceptable results, it is also complex for simple truss-like structures since it requires the solution of two analyses (i.e. EFEA for long members and FEA for short members) in order to fully characterize the dynamics.

Covered comprehensively in the work of Doyle $[17,18]$ in 1989, the spectral finite element method differs from the conventional FEA method in that wave propagation effects are included in the formulation of a finite element. Consequently, there are two major simplifications that arise. First, element subdivisions of structural members for proper mass and stiffness distribution are no longer required, thus reducing the general size of the problem. Continuous single elements between structural discontinuities can be utilized. Second, due to the ability to accurately and efficiently model wave propagation, this computational approach allows for straightforward modal analysis in the mid-frequency range. Thus, the spectral finite element method is exploited throughout the work presented herein and serves as a practical tool for mid-frequency structural dynamic analysis. 


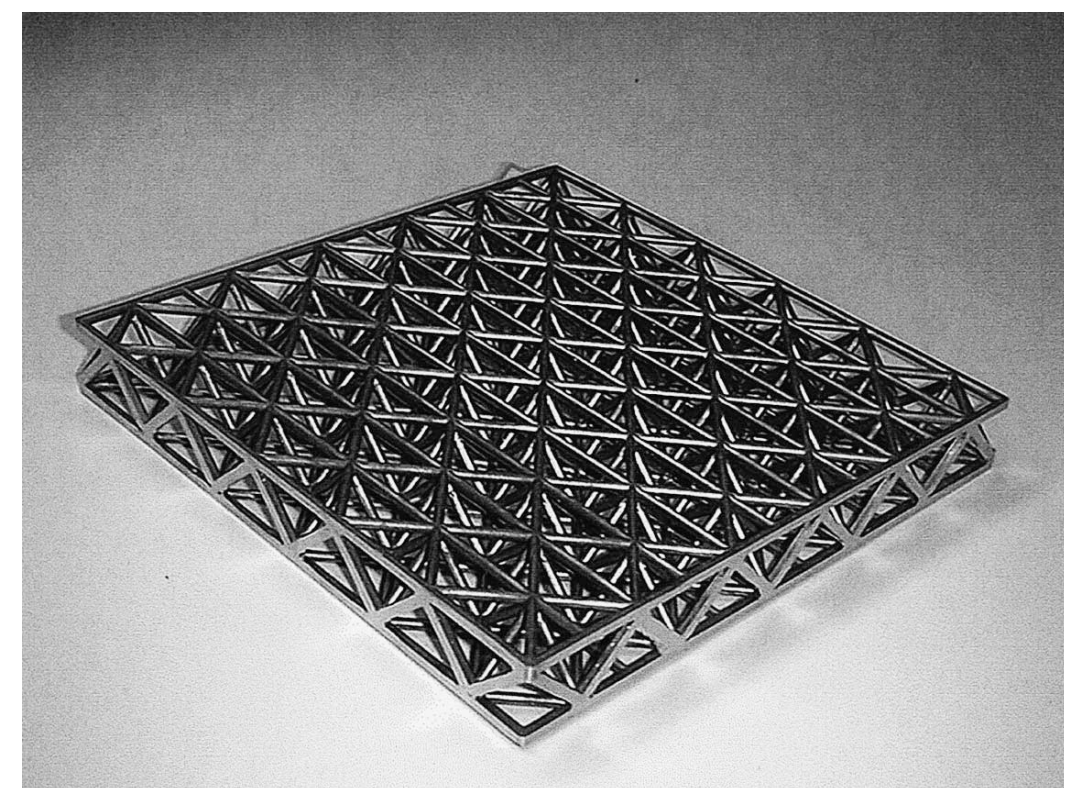

Figure 1.4: 3-D truss made from an aluminum casting alloy, from Wallach \& Gibson $[102]$

\subsubsection{Analysis of Truss / Lattice Structures}

The ability to control either the static or dynamic response of a structure has generated great interest within the structural design community. Particularly, truss structures, sometimes referred to as lattice or cellular structures, have been proven across engineering disciplines to provide a light, strong chassis having multi-functional characteristics that can be utilized in support of more complex machines and systems.

From a static analysis perspective, advances in the design of truss structures are still being made as demonstrated by the recent work of Wang [103] who focused on a unit cell analysis approach for functionally graded structures. Other examples include the work by Bezazi et al. [4] who examined the static properties of a new reentrant honeycomb composite, and Chiras et al. [14] who optimized the structural performance of truss panels with a tetragonal topology. Prototypes of novel designs, as exemplified by the structure in Figure 1.4, are also being manufactured in order to experimentally characterize static response. Various manufacturing processes, such 
as investment casting, are being explored as discussed by Wallach \& Gibson [102] and Wadley [101]. Despite this ever growing body of work, the research field pertaining to the static response of truss structures is, for the most part, well established.

From a dynamic analysis perspective, Brillouin [11], Langley [56], Mead \& Parthan [67], and Noor [73] are but a few researchers over the last century that have studied the basic principles of wave propagation in periodic lattices. Structures with "band gap" characteristics have been identified and become the subject of a great deal of investigation as evidenced by the work of Fish et al. [25], Halkjaer et al. [33], Hussein et al. [43], Martinsson \& Movchan [65], Ruzzene et al. [85], and Sigmund \& Jensen [92]. Such structures have frequency bands in which wave propagation is either hindered (i.e. "stop bands") or unhindered (i.e. "pass bands").

Specific to larger periodic truss beams, Signorelli \& von Flotow [93] used a transfer matrix analysis to establish that these structures exhibit complex mechanical filtering properties when subjected to vibration. Bondaryk [9] also showed, via a direct global stiffness matrix approach, that larger truss structures with varying strut lengths tend to a low-pass frequency characteristic. Furthermore, El-Raheb \& Wagner [20] proposed a transfer matrix method for the evaluation of the vibro-acoustic response of periodic sandwich truss panels. El-Raheb [19] then went on to use this transfer matrix method to examine the frequency response of curved sandwich truss panels having either fluid-filled cavities or visco-elastic constrained layer damping.

In has been shown in much of this prior work that periodic lattices or truss structures can often be analyzed at both "local" and "global" scales. The local level analysis can often predict the global response leading to a reduction in computational effort. This reduction in computational effort then allows for greater freedom in the exploration of alternative designs, the execution of optimization routines, or the 
sizing of prototype structures.

The research presented in this dissertation moves the dynamic analysis of periodic truss structures in a new direction. It is based on similar research performed by Ruzzene [84] who also exploited the aforementioned local-global approach in studying the vibrations of and sound radiation from sandwich beams with periodic truss core. Ruzzene investigated two structures composed of a traditional "square" core topology and a "reentrant" or negative Poisson's ratio core topology, respectively. Thus, Ruzzene's work serves as a seminal framework for this research in three ways. First, Ruzzene demonstrated that truss beams with reentrant core radiated less sound. The deformed shapes of the reentrant core suggested topologies similar to compliant four-bar mechanisms. Second, the spectral finite element method proved to be an efficient technique in the analysis of structures having multiple spatial scales and complicated internal geometry. This is in contrast to the use of the conventional finite element method where many more elements are necessary to properly capture dynamic (i.e. wave propagation) behavior. Third, the spectral or frequency based method proved to be an appropriate means for interfacing between acoustic and structural analyses since sound radiation in an acoustic medium is often analyzed using a Fourier transform process as explained by Junger \& Feit [49] and Stuart [95].

Accordingly, the research presented in this dissertation begins by examining the vibro-acoustic response of simplified two-dimensional (2-D) truss beams with periodic integral compliant mechanisms. A local-global computational approach is similarly employed. This work then leads into the eventual analysis and design of 2-D multilayered lattices, and 3-D structures, having integral compliant mechanisms. 


\subsubsection{Vibration Isolation}

The selection of a vibration isolation technique is dependent upon the nature of the vibratory problem. In most cases the analyst must identify one of three goals: 1) reduction of response at a specific frequency, 2) reduction of response across a broadband frequency range of interest, or 3) precisely controlling response to achieve a desired output. Passive damping techniques are often applied to the first and second goals with relative success. The third goal typically requires active damping techniques as greater precision is necessary. Thus, such goals ultimately dictate the manner in which damping systems are employed and the extent to which they must be utilized.

A fundamental feature of passive damping is that it does not add to the kinetic energy of a structure, but rather passive damping dissipates kinetic energy in response to the structure's motion, per Housner et al. [39]. This form of damping has the benefit of reduced complexity since a power source is not necessary. Additionally, safety is less of a concern since stable systems will remain so. As Faulkner [24], Johnson [47], and Nashif et al. [71] have noted, the passive damping of structures is traditionally based on one of four technologies including visco-elastic materials, viscous fluids, magnetics, or passive piezoelectrics. More recently, alternative ideas have been developed as illustrated by the uniaxial spring-mass vibration isolators proposed by Yilmaz \& Kikuchi [107, 108], which can be designed for low-pass filter, band-stop, or broad-band vibration isolation applications. Regardless, in terms of conventional methods, visco-elastic damping layer treatments are still most common while viscous fluids, magnetics, and piezoelectrics are typically seen in strut embodiments. Furthermore, the implementation of conventional damping techniques varies, however, they share the two disadvantages of added weight and temperature sensi- 


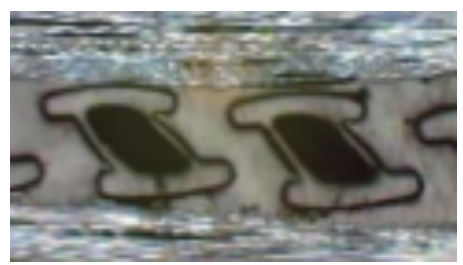

Figure 1.5: Cross-section of machine augmented composite, from Hawkins et al. [37] tivity. Moreover, passive damping is often added as an afterthought to a structure once specific resonance or broadband response problems have been identified. This fact is illustrated by the ISS example given in Section 1.1.

In contrast to passive damping techniques, active damping systems typically use feedback or feedforward control to minimize mechanical vibration through automatic modification of structural response within the control system bandwidth as discussed by Preumont [82] and Fuller et al. [28]. An active system can be lighter and less expensive than a passive one, and in some cases they can achieve performance that would otherwise be impossible (e.g. disturbance correction in earth based telescopes). Despite these advantages, active systems tend to increase complexity since they require power and have the potential to destabilize the system if the control scheme is not properly implemented, per Housner et al. [39].

Moving from a structural viewpoint to a material level perspective, the literature is abundant in the field concerned with designing materials for novel vibration reduction characteristics. Controlling passive vibratory response via compliant, mechanism-like topologies is possible, as established by the negative Poisson's ratio foam developed by Lakes [55] whose dynamic properties were also analyzed by Scarpa [88]. Additionally, an embedded machine patent pertaining to material design is held by Hawkins [36]. Figure 1.5 shows a material developed by Hawkins in which there are numerous embedded 4-bar machines. This work has recently been 
expanded upon by McCutcheon et al. [66] and Tang et al. [97] to demonstrate the potential for achieving novel dynamic functions. However, a unified analysis, design, and optimization methodology using multi-scale compliance for vibro-acoustic dynamic applications is not yet established, and is thus the objective of the research presented in this dissertation.

\subsubsection{Compliant Mechanism Design}

Traditional mechanisms have rigid links with discrete joints while compliant mechanisms have flexible links and joints that achieve the same function of traditional mechanisms through material elasticity. A significant amount of work has been done in the static analysis and synthesis of compliant mechanisms, as demonstrated by Ananthasuresh [2], Frecker [27], Howell [40], Kim [51], Larsen [57], Lobontiu [59], and Saggere [86].

Only lately are the dynamic characteristics of compliant mechanisms being explored more fully by researchers like Yu et al. [109]. In their work they analyzed compliant mechanism dynamic behavior using the pseudo-rigid body model with the goal of determining natural frequencies. Other research in this developing field has focused on the merging of actuators with compliant mechanisms. For example, Tantanawat, Li, \& Kota [98] have shown how a compliant mechanism stroke amplifier can be applied to active vibration isolation systems. Shown in Figure 1.6 is the compliant stroke amplifier that serves as the foundation of their research. This compliant mechanism was based upon a patent for a micro-electro-mechanical (MEM) system originally developed by Hetrick \& Kota [38] and Kota et al. [53].

The work presented here further builds on the study of this stroke amplifier topology. This compliant mechanism was initially selected since a considerable amount of analysis has been performed on it and its general behavior is well characterized. 


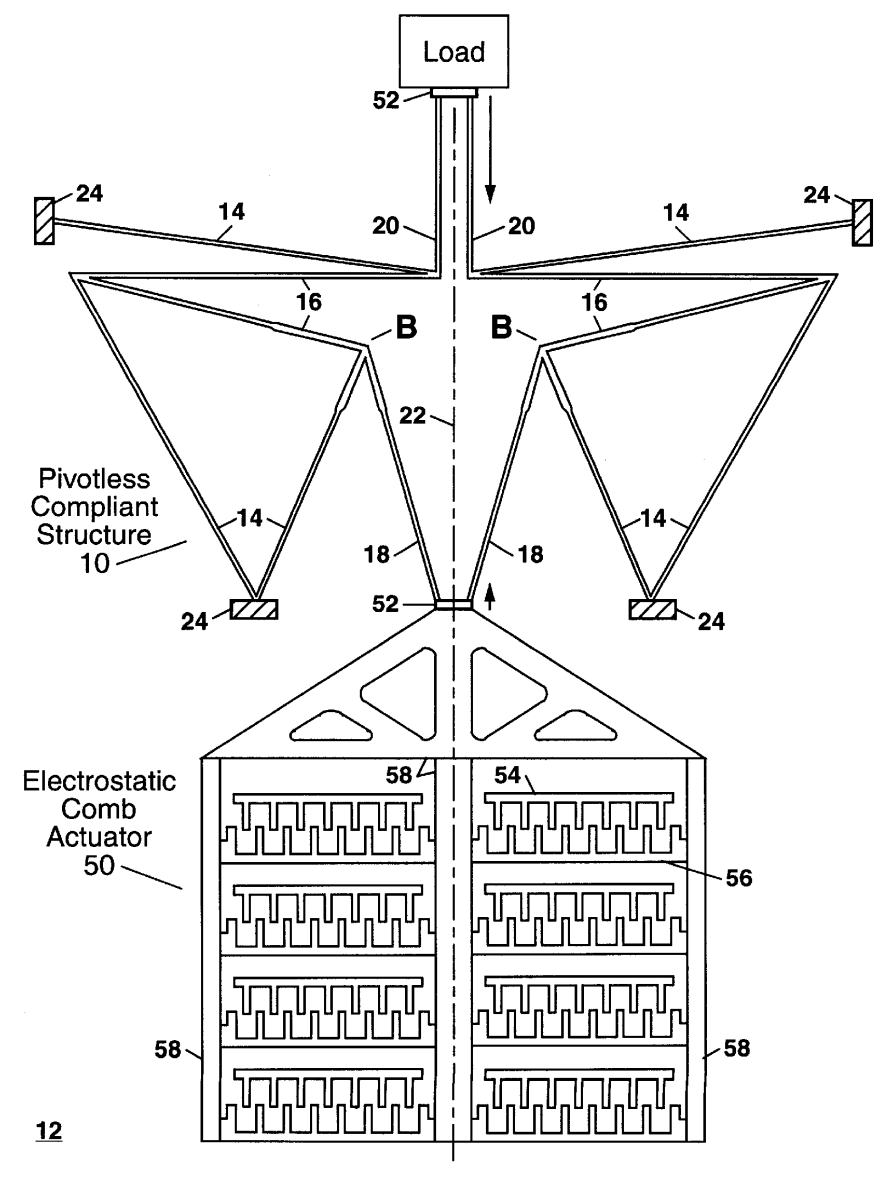

Figure 1.6: Compliant structure MEM device, from Kota et al. [53]

This fact makes it a suitable benchmark. Additionally, while the active control applications mentioned above incorporated system level actuators for the generation mechanism motion, the following work utilizes the notion of vibro-acoustically induced response. Thus, vibration isolation is accomplished passively, via a number of periodic integral compliant mechanisms within a structure, rather than actively. Serving as independent verification of the results achieved using this mechanism, another separate compliant mechanism core topology is also synthesized as an initial step in this research.

Based on this effort, two hypotheses are proposed and investigated with regard to synthesizing unit cell compliant mechanism core topologies. The first hypothesis 
consists of using amplification principles as a guide in the selection of a mechanism topology and shape. The principle of mechanical advantage is utilized; and, as a simplification, power conservation (i.e. the use of rigid links) is assumed. However, since the mechanism is ultimately compliant, power is not conserved between the input and output sides of the structure as suggested by Salamon \& Midha [87]. Thus, using the results from the spectral finite element analysis, the total energy distribution in the structure is visualized along with the deformed shape of the structure in order to provide a complete understanding of energy flow, isolation, and dissipation.

The second hypothesis is based on utilizing the local unit cell length scale coupled with its frequency response function (FRF), as described by Ewins [23], to control response attenuation zones. A straightforward wavenumber-frequency analysis is developed to understand these unit cell length scale effects. In conjunction, the compliant mechanism FRF amplitude and phase information is computed using the results from the spectral finite element analysis in order to gain substantial insight into the behavior of global structures.

Therefore, while the current state of the arts shows that much work has been done to understand standalone static and dynamic compliant mechanism performance, this research takes a different step forward by utilizing multiple small scale compliant mechanisms within larger scale structures for novel dynamic functionality. The aforementioned principle of mechanical advantage, deformed shape and total energy mapping, unit cell wavenumber analysis, and FRF analysis represent an innovative assemblage of tools used in forming an in-depth understanding of the fundamental structural dynamics at work. 


\subsubsection{Structural Optimization}

In this final section of Chapter 1, structural optimization is introduced as a rich field that complements the four research areas discussed above. Specifically, optimization using an evolutionary (e.g. genetic) algorithm is investigated. As Goldberg [30] outlines, the efficiency of a genetic algorithm comes from the fact that while randomized, it uses historical information based on design fitness to search new spaces. A genetic algorithm is capable of handling noisy data in a global search. While calculus-based methods are generally limited to searching for local maxima and minima, a genetic algorithm can search larger design spaces for an optimal solution. Thus, genetic algorithms have a notable advantage in optimizing truss-like structures that have a large number of non-linear, inter-related variables that produce a broadband frequency response with many resonant peaks. These are the primary reasons why a genetic algorithm was chosen over a calculus-based method.

Many researchers have applied genetic algorithms to topology optimization. Chapman, Saitou, \& Jakiela [13] examined the optimization of a cantilevered beam as represented by a finely discretized continuum. Parsons \& Canfield [77] explored the genetic algorithm optimization of truss structures for compliant mechanism applications, and they validated the approach on familiar examples like a compliant gripper. These researchers also verified that genetic algorithms work for shape optimization by allowing nodes within a compliant mechanism structure to wander once a final topology had been selected. In this way, even better performance of the compliant mechanism is achievable. Shape optimization has further been examined in relationship to truss structures with unusual geometries as investigated by Keane [50] and Moshrefi-Torbati et al. [68]. Two optimized truss structures developed by Keane and Moshrefi-Torbati et al., that have enhanced vibratory characteristics, are shown in 


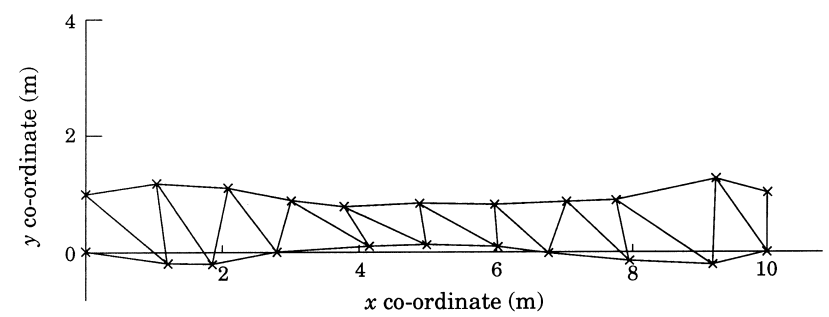

Figure 1.7: Optimized 2-D truss structure, from Keane [50]

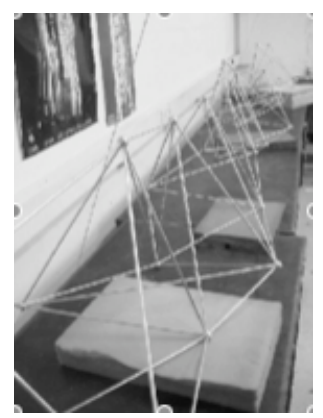

Figure 1.8: Optimized 3-D truss structure, from Moshrefi-Torbati et al. [68]

Figures 1.7 and 1.8. The majority of this earlier research suggests that the best first approximations to optimized structures can be obtained in a straightforward manner using already established genetic algorithm methods.

Given this, the hypotheses and tools proposed in the Section 1.2.4 are used as a guide in selecting two a priori mechanism topologies and shapes. From there, the final size optimization of a compliant mechanism design is examined with the goal of "tuning" specific performance criteria. This entails coupling the spectral finite element analysis discussed in Section 1.2.2 with a basic selection, crossover, and mutation algorithm patterned after the work by Satyadas and Krishnakumar in Winter [104]. This preliminary study does not exhaust all possibilities with regard to the implementation of a genetic algorithm for optimization, but rather it exemplifies the power of such an approach.

In subsequent steps, the topology optimization of a simple unit cell binary ground structure truss network for vibro-acoustic response is investigated. This study pro- 
vides additional insight into possible compliant mechanism unit cell designs. The strategy used in selecting final topologies is similar that used by Maddisetty \& Frecker [64] where structural elements are allowed to range in thickness from a predetermined upper limit to a lower limit approaching zero. Elements that lay in the optimization domain of the structure and are close to zero thickness are then assumed to contribute minimally to the performance of the unit cell allowing their removal from the structure. The final optimized topologies are then revealed.

Observe that this strategy is not as comprehensive as the load path representation method proposed by Lu \& Kota [61]. The elimination of elements to form final topologies based on a predetermined minimum element thickness does not fully avoid binary ground structure issues associated with floating structures, ungrounded designs, and disconnected inputs. Regardless of these drawbacks, the approach taken here illustrates that additional unit cell topologies are realizable and opens the door to further research implementing more sophisticated genetic algorithm routines. 


\section{CHAPTER 2}

\section{Computational Modeling}

In support of the goals set forth in Chapter 1, a computational code was developed for the implementation of the spectral finite element method. A brief review of the conventional formulation of a 2-D plane frame finite element is given in Section 2.1 prior to outlining the theory behind the spectral finite element method in Section 2.2. A 2-D analysis domain serves as a basic building block for more complicated problems and has been the proving ground for numerous compliant mechanism analyses. However, this research is not limited to two dimensions and the spectral finite element computational code is extended to handle 3-D space frame elements in Section 2.3.

After the introduction of the spectral finite element method, an assessment of the accuracy of the computational code is made using a simple wave propagation problem consisting of a $2-\mathrm{D}$ semi-infinite cantilevered beam subject to an impulse. This problem was proposed by Doyle \& Farris [18] and their solution serves as a benchmark in Section 2.4. In Section 2.5 the code is then further verified using known solutions for the natural frequencies of free vibration for a fixed-fixed beam given by Blevins [8]. 


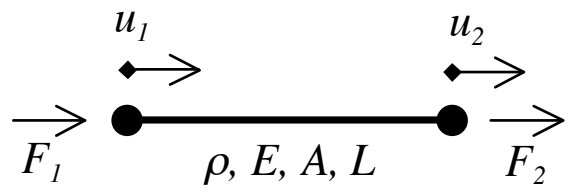

Rod (2 DOF Total)

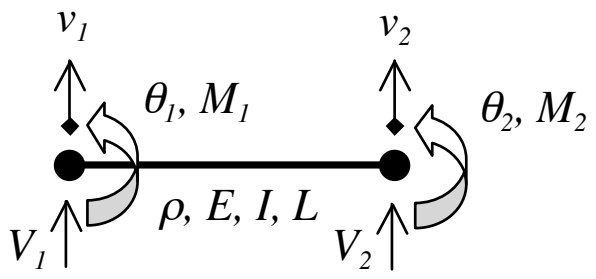

Beam (4 DOF Total)

Figure 2.1: Rod element and beam element nodal degrees of freedom and loads

\subsection{Conventional Finite Element Formulation: 2-D Rod \& Beam}

A conventional linear frame finite element combines the degrees of freedom of both a rod element and a beam element as outlined by Yang [106]. In Figure 2.1, a rod element is shown to have a single axial degree of freedom at each node while a beam element is shown to have both transverse and rotational degrees of freedom at each node.

The element material density, Young's modulus, cross-sectional area, length, and area moment of inertia are denoted by $\rho, E, A, L$, and $I$, respectively. The rod element axial loads and displacements are specified respectively by $F_{i}$ and $u_{i}$, while the beam transverse loads, moments, transverse displacements, and rotations are given respectively by $V_{i}, M_{i}, v_{i}$, and $\theta_{i}$, where $i$ denotes the node number of interest.

An energy formulation of the rod element stiffness matrix is given below. For the rod element, the axial displacement at any location, $x$, along the length of the element is given in terms of the nodal displacements as

$$
u(x)=\left(1-\frac{x}{L}\right) u_{1}+\left(\frac{x}{L}\right) u_{2}
$$

where the coefficients in front of the nodal displacements are the nodal shape func- 
tions, $N_{1}$ and $N_{2}$, which can be written explicitly as

$$
N_{1}=1-\frac{x}{L} \quad ; \quad N_{2}=\frac{x}{L}
$$

The axial strain, $\varepsilon_{x}$, and stress, $\sigma_{x}$, of the element are then expressed in the following form

$$
\varepsilon_{x}=\frac{\partial u}{\partial x} \quad ; \quad \sigma_{x}=E \varepsilon_{x}
$$

and assuming a constant element cross-section, the strain energy, $U$, of an element, $e$, follows from the definition

$$
U_{e}=\frac{1}{2} \int_{0}^{L} \int_{A} \sigma_{x} \varepsilon_{x} d A d x=\frac{1}{2} \int_{0}^{L} E A\left(\frac{d u}{d x}\right)^{2} d x
$$

Using Eqn (2.1), Eqn (2.4) can be evaluated and written in matrix form as

$$
U_{e}=\frac{1}{2}\left[\begin{array}{ll}
u_{1} & u_{2}
\end{array}\right] \frac{E A}{L}\left[\begin{array}{cc}
1 & -1 \\
-1 & 1
\end{array}\right]\left[\begin{array}{l}
u_{1} \\
u_{2}
\end{array}\right]=\frac{1}{2} \mathbf{d}_{\mathrm{r}}^{\mathrm{T}} \mathbf{K}_{\mathrm{r}} \mathbf{d}_{\mathrm{r}}
$$

where $\mathbf{K}_{\mathrm{r}}$ is the rod element stiffness matrix and $\mathbf{d}_{\mathrm{r}}$ is the rod element nodal displacement vector.

The work, $W$, done by the applied force to the element can also be defined in terms of the rod element nodal force vector, $\mathbf{F}_{\mathrm{r}}$, as

$$
W_{e}=\mathbf{d}_{\mathrm{r}}^{\mathrm{T}} \mathbf{F}_{\mathrm{r}}
$$

Combining Eqn (2.5) with Eqn (2.6), the total potential energy of the element is formed using the expression

$$
\Pi_{e}=U_{e}-W_{e}=\frac{1}{2} \mathbf{d}_{\mathrm{r}}^{\mathrm{T}} \mathbf{K}_{\mathrm{r}} \mathbf{d}_{\mathrm{r}}-\mathbf{d}_{\mathrm{r}}^{\mathrm{T}} \mathbf{F}_{\mathrm{r}}
$$

The principle of minimum potential energy is then stated for the rod element in equation form as

$$
\frac{\partial \prod_{e}}{\partial \mathbf{d}_{\mathrm{r}}}=0
$$


Rod

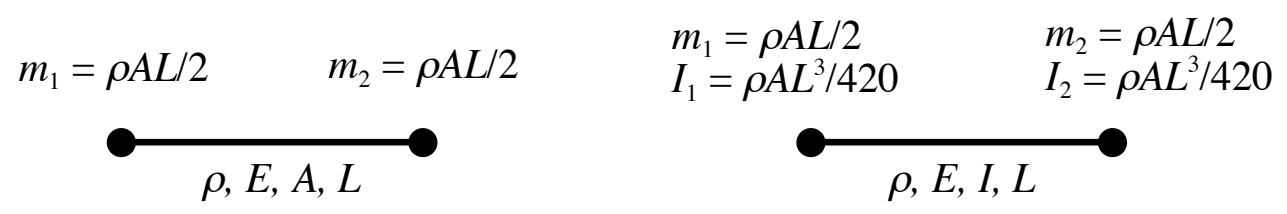

Figure 2.2: Rod element and beam element lumped-mass approximation

Thus, Eqn (2.8) yields the rod element nodal force vector in terms of the rod element stiffness matrix and nodal displacement vector

$$
\begin{gathered}
{\left[\begin{array}{c}
F_{1} \\
F_{2}
\end{array}\right]=\frac{E A}{L}\left[\begin{array}{cc}
1 & -1 \\
-1 & 1
\end{array}\right]\left[\begin{array}{l}
u_{1} \\
u_{2}
\end{array}\right]} \\
\mathbf{F}_{\mathrm{r}}=\mathbf{K}_{\mathrm{r}} \mathbf{d}_{\mathrm{r}}
\end{gathered}
$$

In the development of a conventional finite element, it is often assumed that the mass of the element acts as inertia forces lumped at the nodes instead of being distributed over the length of the element. This is known as the lumped mass method. This method is derived from the consistent mass method, which is based on a kinetic energy formulation that does not have a simple interpretation of masses at nodes. Both methods are shown to produce results that are roughly equivalent in terms of accuracy, although the lumped mass matrix has the computational advantage of being diagonal. Consequently, a lumped mass approximation is reasonable and used here in the development of mass matrices for both the rod element and beam element. In Figure 2.2 the lumped mass is given by $m_{i}$ and the mass moment of inertia is given by $I_{i}$ at each node of interest. 
Thus, using Newton's second law of motion, the nodal inertia forces, $F_{i}{ }^{\prime}$, on the rod element are written in terms of the nodal acceleration, $\ddot{u}_{i}$, as follows

$$
\begin{aligned}
& F_{1}^{\prime}=m_{1} \frac{\partial^{2} u_{1}}{\partial t^{2}}=\frac{\rho A L}{2} \ddot{u}_{1} \\
& F_{2}^{\prime}=m_{2} \frac{\partial^{2} u_{2}}{\partial t^{2}}=\frac{\rho A L}{2} \ddot{u}_{2}
\end{aligned}
$$

Eqns (2.11) and (2.12) can be written to express the rod element inertia force vector, $\mathbf{F}_{\mathrm{r}}{ }^{\prime}$, in matrix form as a function of the rod element mass matrix, $\mathbf{M}_{\mathrm{r}}$, and nodal acceleration vector, $\ddot{\mathbf{d}}_{\mathrm{r}}$, as

$$
\begin{gathered}
{\left[\begin{array}{c}
F_{1}^{\prime} \\
F_{2}^{\prime}
\end{array}\right]=\frac{\rho A L}{2}\left[\begin{array}{cc}
1 & 0 \\
0 & 1
\end{array}\right]\left[\begin{array}{c}
\ddot{u}_{1} \\
\ddot{u}_{2}
\end{array}\right]} \\
\mathbf{F}_{\mathrm{r}}{ }^{\prime}=\mathbf{M}_{\mathrm{r}} \ddot{\mathbf{d}}_{\mathrm{r}}
\end{gathered}
$$

Combining Eqn (2.10) with Eqn (2.14) yields the homogeneous system of differential equations for the rod element.

$$
0=\mathbf{F}_{\mathrm{r}}{ }^{\prime}+\mathbf{F}_{\mathrm{r}}=\mathbf{M}_{\mathrm{r}} \ddot{\mathbf{d}}_{\mathrm{r}}+\mathbf{K}_{\mathrm{r}} \mathbf{d}_{\mathrm{r}}
$$

For forced motion, the non-homogeneous system of differential equations for dynamic applications of the rod element is then expressed in terms of the externally applied axial force vector, $\mathbf{F}_{\text {ext_r }}$, and Eqn (2.15) as

$$
\mathbf{F}_{\text {ext_r }}=\mathbf{M}_{\mathrm{r}} \ddot{\mathbf{d}}_{\mathrm{r}}+\mathbf{K}_{\mathrm{r}} \mathbf{d}_{\mathrm{r}}
$$

A similar procedure to the one just taken for the rod element can also be followed to arrive at the commonly known beam element stiffness matrix, $\mathbf{K}_{\mathrm{b}}$, and lumped 
mass matrix, $\mathbf{M}_{\mathrm{b}}$. The details of the derivation are omitted for the sake of brevity.

$$
\begin{gathered}
\mathbf{K}_{\mathrm{b}}=\frac{E I}{L^{3}}\left[\begin{array}{cccc}
12 & 6 L & -12 & 6 L \\
6 L & 4 L^{2} & -6 L & 2 L^{2} \\
-12 & -6 L & 12 & -6 L \\
6 L & 2 L^{2} & -6 L & 4 L^{2}
\end{array}\right] \\
\mathbf{M}_{\mathrm{b}}=\frac{\rho A L}{420}\left[\begin{array}{cccc}
210 & 0 & 0 & 0 \\
0 & L^{2} & 0 & 0 \\
0 & 0 & 210 & 0 \\
0 & 0 & 0 & L^{2}
\end{array}\right]
\end{gathered}
$$

These matrices allow for the non-homogeneous system of differential equations for dynamic applications of the beam element to be written as

$$
\mathbf{F}_{\text {ext_b }}=\mathbf{F}_{\mathrm{b}}{ }^{\prime}+\mathbf{F}_{\mathrm{b}}=\mathrm{M}_{\mathrm{b}} \ddot{\mathbf{d}}_{\mathrm{b}}+\mathbf{K}_{\mathrm{b}} \mathbf{d}_{\mathrm{b}}
$$

where $\mathbf{F}_{\text {ext_b }}$ is the externally applied transverse force and moment vector; $\mathbf{F}_{\mathrm{b}}{ }^{\prime}$ is the nodal inertia force vector; $\mathbf{F}_{\mathrm{b}}$ is the nodal force vector; $\ddot{\mathbf{d}}_{\mathrm{b}}$ is the nodal acceleration vector; and $\mathbf{d}_{\mathrm{b}}$ is the nodal displacement vector for the beam element.

A general frame member subject to both axial and bending loads can now be formulated by assembling the rod element stiffness and mass matrices with the beam element stiffness and mass matrices, respectively. The resulting $6 \times 6$ frame element stiffness matrix, $\mathbf{K}_{\mathrm{f}}$, and the $6 \times 6$ frame element mass matrix, $\mathbf{M}_{\mathrm{f}}$, reflect the 
respective load-deflection relation and inertia properties of a general frame member.

$$
\mathbf{K}_{\mathrm{f}}=\left[\begin{array}{cccccc}
\frac{E A}{L} & 0 & 0 & -\frac{E A}{L} & 0 & 0 \\
0 & \frac{12 E I}{L^{3}} & \frac{6 E I}{L^{2}} & 0 & -\frac{12 E I}{L^{3}} & \frac{6 E I}{L^{2}} \\
0 & \frac{6 E I}{L^{2}} & \frac{4 E I}{L} & 0 & -\frac{6 E I}{L^{2}} & \frac{2 E I}{L} \\
-\frac{E A}{L} & 0 & 0 & \frac{E A}{L} & 0 & 0 \\
0 & -\frac{12 E I}{L^{3}} & -\frac{6 E I}{L^{2}} & 0 & \frac{12 E I}{L^{3}} & -\frac{6 E I}{L^{2}} \\
0 & \frac{6 E I}{L^{2}} & \frac{2 E I}{L} & 0 & -\frac{6 E I}{L^{2}} & \frac{4 E I}{L}
\end{array}\right]
$$

Accordingly, the inertia force vector, $\mathbf{F}_{\mathrm{f}}{ }^{\prime}$, nodal force vector, $\mathbf{F}_{\mathrm{f}}$, acceleration vector, $\ddot{\mathbf{d}}_{\mathrm{f}}$, and displacement vector, $\mathbf{d}_{\mathrm{f}}$, for a frame element are

$$
\mathbf{F}_{\mathrm{f}}^{\prime}=\left[\begin{array}{c}
F_{1}^{\prime} \\
V_{1}^{\prime} \\
M_{1}^{\prime} \\
F_{2}^{\prime} \\
V_{2}^{\prime} \\
M_{2}^{\prime}
\end{array}\right] ; \quad \mathbf{F}_{\mathrm{f}}=\left[\begin{array}{c}
F_{1} \\
V_{1} \\
M_{1} \\
F_{2} \\
V_{2} \\
M_{2}
\end{array}\right] ; \quad \ddot{\mathbf{d}}_{\mathrm{f}}=\left[\begin{array}{c}
\ddot{u}_{1} \\
\ddot{v}_{1} \\
\ddot{\theta}_{1} \\
\ddot{u}_{2} \\
\ddot{v}_{2} \\
\ddot{\theta}_{2}
\end{array}\right] ; \quad \mathbf{d}_{\mathrm{f}}=\left[\begin{array}{c}
u_{1} \\
v_{1} \\
\theta_{1} \\
u_{2} \\
v_{2} \\
\theta_{2}
\end{array}\right]
$$

In the analysis of a structure composed of many frame elements the remaining computational step is to assemble elements in the traditional manner where the 
element matrices in the local coordinate system are transformed to a global coordinate system by a standard transformation (i.e. rotation) matrix. The components from individual frame element stiffness and mass matrices that correspond to specific global degrees of freedom are assembled into the global stiffness and mass matrices, $\mathbf{K}_{\mathrm{G}}$ and $\mathbf{M}_{\mathrm{G}}$, respectively, per the usual numerical algorithms outlined in references such as Hughes [42]. The application of boundary conditions then follows to reduce the total number of global degrees of freedom.

Following this, the reduced system of differential equations representing the forced motion response of a structure modeled with frame members is obtained

$$
\mathbf{F}_{\text {ext_G }}=\mathbf{F}_{\mathrm{G}}{ }^{\prime}+\mathbf{F}_{\mathrm{G}}=\mathbf{M}_{\mathrm{G}} \ddot{\mathbf{d}}_{\mathrm{G}}+\mathbf{K}_{\mathrm{G}} \mathbf{d}_{\mathrm{G}}
$$

where $\mathbf{F}_{\text {ext_G }}$ is the externally applied nodal force vector; $\mathbf{F}_{\mathrm{G}}{ }^{\prime}$ is the nodal inertia force vector; $\mathbf{F}_{\mathrm{G}}$ is the nodal force vector; $\ddot{\mathbf{d}}_{\mathrm{G}}$ is the nodal acceleration vector; and $\mathbf{d}_{\mathrm{G}}$ is the nodal displacement vector for the global structure.

Two restrictions are evident for structures modeled with conventional finite elements. First, for problems solved in the frequency domain (e.g. modal analysis), a prohibitively large number of elements must be used in order to accurately represent the mass and stiffness distribution of each structural member. This becomes especially important in wave propagation problems where the element discretization often must be fine enough to capture the numerous bending modes of a structure at higher frequencies. More elements lead to a higher-order characteristic equation that must be solved in the eigenvalue analysis to determine the larger number of resonances. This requires greater computational effort. A second restriction arises when solving dynamic problems in the time domain. Specifically, a step-by-step numerical integration scheme, such as the Newmark method outlined in Hughes [42], is typically implemented. However, in order to capture wave propagation phenomena 
at high frequencies, the time step over which the system of equations is repeatedly solved must be sufficiently small. This again leads to excessive computational times for large systems. Thus, such limitations have made conventional FEA of structural dynamic response in the middle to high frequency range computationally expensive.

\subsection{Spectral Finite Element Formulation: 2-D Rod \& Beam}

In the development of a spectral rod element the mass is no longer considered lumped at the ends of the element, but rather distributed evenly along the length. This treatment of the mass distribution allows for the propagation of longitudinal waves through the rod element acting as a waveguide. It can be established, as shown by Doyle [17], that the space-time solution for the longitudinal displacement of a point on a rod is represented by

$$
u(x, t)=\sum_{n} \hat{u}_{n}\left(x, \omega_{n}\right) \mathrm{e}^{\mathrm{i} \omega_{n} t}
$$

Eqn (2.24) represents the general solution for the time harmonic variation of any variable, where $\omega$ is the frequency and Eqn (2.24) is summed over the $n$, or number of frequencies of interest. In this equation, the ${ }^{\wedge}$ nomenclature is established to represent a spectral quantity. Thus, the spectral displacements for the rod element are represented by $\hat{u}_{n}$, which have the form

$$
\hat{u}_{n}=A \mathrm{e}^{-\mathrm{i} k_{n} x}+B \mathrm{e}^{-\mathrm{i} k_{n}(L-x)} \quad ; \quad k_{n}=\omega_{n} \sqrt{\frac{\rho}{E}}
$$

Comparing Eqn (2.25) with Eqn (2.1) reveals this to be the frequency dependent rod element shape function. Observe that the wavenumber, $k_{n}$, not only depends on frequency, but also the material through which longitudinal waves are traveling. In the equations that follow, the subscript $n$ is dropped, however still implied. 
The coefficients $A$ and $B$ can now be solved for in terms of the nodal displacements by applying the known boundary conditions

$$
\hat{u}\left(x=x_{1}, \omega\right)=\hat{u}_{1}
$$

$$
\hat{u}\left(x=x_{2}, \omega\right)=\hat{u}_{2}
$$

$$
\text { where, } \quad x_{1}=0 \quad ; \quad x_{2}=L
$$

Consequently,

$$
\left[\begin{array}{l}
A \\
B
\end{array}\right]=\frac{1}{1-\mathrm{e}^{-\mathrm{i} 2 k L}}\left[\begin{array}{cc}
1 & -\mathrm{e}^{-\mathrm{i} k L} \\
-\mathrm{e}^{-\mathrm{i} k L} & 1
\end{array}\right]\left[\begin{array}{c}
\hat{u}_{1} \\
\hat{u}_{2}
\end{array}\right]
$$

The nodal forces for a rod element are then found in terms of the element stiffness matrix as follows

$$
\hat{F}_{i}=\left.\mathrm{n}_{i} E A \frac{d \hat{u}}{d x}\right|_{x=x_{i}} \quad \text { where, } \quad \mathrm{n}_{i}=\left\{\begin{array}{r}
-1 \text { for } i=1 \\
1 \text { for } i=2
\end{array}\right.
$$

Resulting in

$$
\begin{aligned}
& \hat{F}_{1}=-\left.E A \frac{d \hat{u}}{d x}\right|_{x=x_{1}} \\
& \hat{F}_{2}=\left.E A \frac{d \hat{u}}{d x}\right|_{x=x_{2}}
\end{aligned}
$$

Or, in matrix form

$$
\left[\begin{array}{c}
\hat{F}_{1} \\
\hat{F}_{2}
\end{array}\right]=E A \frac{\mathrm{i} k}{1-\mathrm{e}^{-\mathrm{i} 2 k L}}\left[\begin{array}{cc}
1+\mathrm{e}^{-\mathrm{i} 2 k L} & -2 \mathrm{e}^{-\mathrm{i} k L} \\
-2 \mathrm{e}^{-\mathrm{i} k L} & 1+\mathrm{e}^{-\mathrm{i} 2 k L}
\end{array}\right]\left[\begin{array}{c}
\hat{u}_{1} \\
\hat{u}_{2}
\end{array}\right]
$$

$$
\hat{\mathbf{F}}_{\mathrm{r}}=\hat{\mathbf{K}}_{\mathrm{r}} \hat{\mathbf{d}}_{\mathrm{r}}
$$


The development of the beam spectral element follows similarly that of the rod element. Bending degrees of freedom, however, allow for the transmission of flexural or transverse wave propagation through the element as opposed to the longitudinal waves addressed by the rod formulation. Hence, the space-time solution for the transverse displacement of a point on a beam element is as follows

$$
v(x, t)=\sum_{n} \hat{v}_{n}\left(x, \omega_{n}\right) \mathrm{e}^{\mathrm{i} \omega_{n} t}
$$

The frequency dependent spectral displacements and wavenumber for the beam element can now be written as

$$
\hat{v}_{n}=C \mathrm{e}^{-\mathrm{i} k_{n} x}+D \mathrm{e}^{-k_{n} x}+F \mathrm{e}^{-\mathrm{i} k_{n}(L-x)}+G \mathrm{e}^{-k_{n}(L-x)} \quad ; \quad k_{n}=\sqrt{\omega_{n}}\left[\frac{\rho A}{E I}\right]^{1 / 4}
$$

Based on this displacement function, the rotation at each end of the beam can be found by taking the derivative of Eqn (2.36) with respect to $x$.

$$
\hat{\phi}=\frac{d \hat{v}}{d x}
$$

Applying the known boundary conditions on the element allows for the solution of the coefficients $C, D, F$, and $G$.

$$
\begin{gathered}
\hat{v}\left(x=x_{1}, \omega\right)=\hat{v}_{1} \\
\hat{\phi}\left(x=x_{1}, \omega\right)=\hat{\phi}_{1} \\
\hat{v}\left(x=x_{2}, \omega\right)=\hat{v}_{2} \\
\hat{\phi}\left(x=x_{2}, \omega\right)=\hat{\phi}_{2}
\end{gathered}
$$


Consequently,

$$
\left[\begin{array}{c}
C \\
D \\
F \\
G
\end{array}\right]=\hat{\mathbf{Q}}\left[\begin{array}{c}
\hat{v}_{1} \\
\hat{\phi}_{1} \\
\hat{v}_{2} \\
\hat{\phi}_{2}
\end{array}\right]
$$

The matrix $\hat{\mathbf{Q}}$ is not given here for the sake of brevity, but easily obtained using symbolical computation software. The beam nodal loads are then found, in terms of the spectral stiffness matrix, by substituting the coefficients $C, D, F$, and $G$ back into Eqn (2.36) and differentiating the resulting displacement function to form the moment and shear force at each node

$$
\begin{gathered}
\hat{V}_{i}=\left.\mathrm{n}_{i} E I \frac{d^{3} \hat{v}}{d x^{3}}\right|_{x=x_{i}} \\
\hat{M}_{i}=-\left.\mathrm{n}_{i} E I \frac{d^{2} \hat{v}}{d x^{2}}\right|_{x=x_{i}}
\end{gathered}
$$

Resulting in

$$
\begin{gathered}
\hat{V}_{1}=\left.E I \frac{d^{3} \hat{v}}{d x^{3}}\right|_{x=x_{1}} \\
\hat{M}_{1}=-\left.E I \frac{d^{2} \hat{v}}{d x^{2}}\right|_{x=x_{1}} \\
\hat{V}_{2}=-\left.E I \frac{d^{3} \hat{v}}{d x^{3}}\right|_{x=x_{2}} \\
\hat{M}_{2}=\left.E I \frac{d^{2} \hat{v}}{d x^{2}}\right|_{x=x_{2}}
\end{gathered}
$$

Or, in matrix form

$$
\hat{\mathbf{F}}_{\mathrm{b}}=\hat{\mathbf{K}}_{\mathrm{b}} \hat{\mathbf{d}}_{\mathrm{b}}
$$


Further details regarding the derivation of the spectral stiffness matrix for the beam element can be found in Appendix A.

There is a boundary condition, which is unique to wave propagation problems when solved using the spectral method, where a finite element member extends to infinity. Such elements are designated as "throw-off" elements by Doyle $[17,18]$ since energy is guided out of the system via these elements. One cannot simply allow the element length to become extremely large since the original spectral stiffness matrix formulation accounts for incoming (i.e. reflected) waves. Rather, one must use a spectral stiffness matrix form for the rod element and beam element where only outgoing waves are addressed.

For the rod throw-off element

$$
\hat{F}_{1}=E A[\mathrm{i} k] \hat{u}_{1}
$$

and for the beam throw-off element

$$
\left[\begin{array}{c}
\hat{V}_{1} \\
\hat{M}_{1}
\end{array}\right]=E I\left[\begin{array}{cc}
(\mathrm{i}-1) k^{3} & \mathrm{i} k^{2} \\
\mathrm{i} k^{2} & (\mathrm{i}+1) k
\end{array}\right]\left[\begin{array}{c}
\hat{v}_{1} \\
\hat{\phi}_{1}
\end{array}\right]
$$

Observe that the form of both of these equations corresponds to right-handed throwoff elements (i.e. elements that extend to infinity to the right), and that it is possible to formulate left-handed elements as well.

At this point, the spectral frame finite element is formulated in a manner similar to that of the conventional frame finite element. The $2 \times 2$ matrix, $\hat{\mathbf{K}}_{\mathrm{r}}$, of the spectral rod element and $4 \times 4$ matrix, $\hat{\mathbf{K}}_{\mathrm{b}}$, of the spectral beam element can be combined to describe a 2-D spectral frame element with three (i.e. longitudinal, transverse, and rotational) degrees of freedom per node. The resulting dynamic stiffness matrix, $\hat{\mathbf{K}}_{\mathrm{f}}$, is $6 \times 6$. The primary advantage of developing the spectral frame element in 
this manner is that all of the tools from the conventional formulation, with regard to assembling elements to form a global structure, can also be applied here.

There are, however, two main differences with the conventional formulation. First, the step-by-step time integration problem from the conventional formulation now becomes a step-by-step frequency problem. Instead of solving a differential equation in time, a pseudo-static equation in frequency must be solved. Second, fewer elements can be used since wave propagation is treated more precisely in this formulation. Thus, the analyst does not have to be as concerned with mesh discretization. In fact, the power of this method is that it is very accurate at high frequencies, as explained by Doyle [17].

In contrast, the primary disadvantage of this method is that the stiffness matrix must be formed and inverted at each frequency, which adds cost to computational efficiency. Post processing can also take more time since fewer nodal displacements are calculated in general. Thus, plotting deformed shapes in detail can be more involved since it requires inputting the known nodal displacements into Eqn (2.25) and Eqn (2.36) and subsequently calculating the desired displacements at an intermediate point of interest along the length of the element.

\subsection{Spectral Finite Element Formulation: 3-D Space Frame}

The 2-D plane frame element formulation presented in Section 2.2 is now readily extended to a 3-D space frame formulation by adding the necessary torsional and out-of-plane bending degrees of freedom. Small displacement theory dictates that all of the axial, bending, and torsional effects can be assumed to be uncoupled. Thus, by combining the equations that treat these effects separately, the 3 -D element formulation is obtained. 


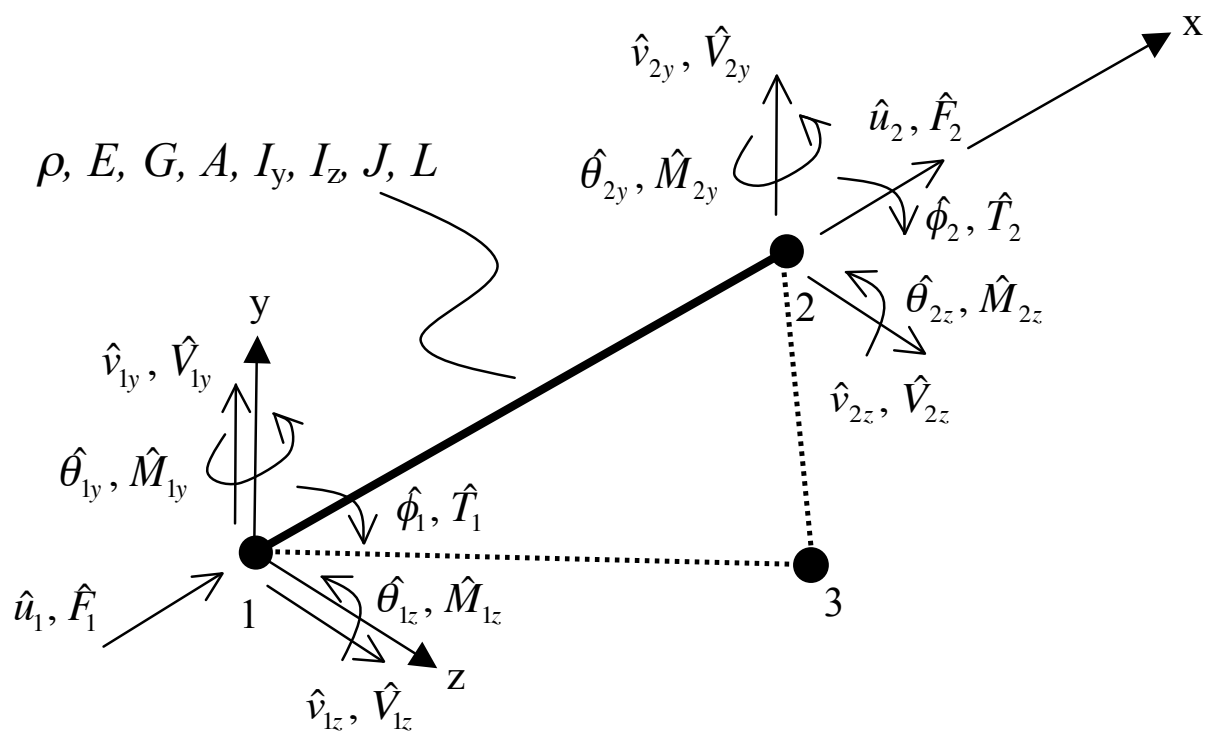

Figure 2.3: 3-D space frame element nodal degrees of freedom and loads

Shown in Figure 2.3 is a space frame element having six degrees of freedom per node. The axial displacement, transverse displacement along the local $y$-axis, transverse displacement along the local $z$-axis, torsional rotation about the local $x$-axis, bending rotation about the local $y$-axis, and bending rotation about the local $z$-axis are given by $\hat{u}_{i}, \hat{v}_{i y}, \hat{v}_{i z}, \hat{\phi}_{i}, \hat{\theta}_{i y}$, and $\hat{\theta}_{i z}$, respectively. The corresponding axial force, transverse force along the local $y$-axis, transverse force along the local $z$-axis, torque about the local $x$-axis, moment about the local $y$-axis, and moment about the local $z$-axis are respectively denoted by $\hat{F}_{i}, \hat{V}_{i y}, \hat{V}_{i z}, \hat{T}_{i}, \hat{M}_{i y}$, and $\hat{M}_{i z}$.

For the space frame element, the three-node method is used to establish all three local element coordinates, per Bhatti [7]. To start, it is assumed that the local $x$-axis vector is defined as pointing in a direction from the first node to the second node of the element. Next, by specifying an orientation vector directed from node 1 to 3, the local $z-x$ plane is arbitrarily defined as shown in Figure 2.3. The local $y$-axis is then found by taking the cross product of the orientation vector with the local 
$x$-axis. Lastly, the local $z$-axis is found by taking the cross product the local $x$-axis with the local $y$-axis. This is a standard convention that serves to correctly orient the space frame element.

The added stiffness terms due to the torsional degrees of freedom are found using a similar derivation to that for the spectral rod element. The equation of motion in matrix form is similar to Eqn (2.33) and can be shown to have the form

$$
\begin{gathered}
{\left[\begin{array}{c}
\hat{T}_{1} \\
\hat{T}_{2}
\end{array}\right]=G J \frac{\mathrm{i} k}{1-\mathrm{e}^{-\mathrm{i} 2 k L}}\left[\begin{array}{cc}
1+\mathrm{e}^{-\mathrm{i} 2 k L} & -2 \mathrm{e}^{-\mathrm{i} k L} \\
-2 \mathrm{e}^{-\mathrm{i} k L} & 1+\mathrm{e}^{-\mathrm{i} 2 k L}
\end{array}\right]\left[\begin{array}{c}
\hat{\phi}_{1} \\
\hat{\phi}_{2}
\end{array}\right]} \\
\hat{\mathbf{F}}_{\mathrm{t}}=\hat{\mathbf{K}}_{\mathrm{t}} \hat{\mathbf{d}}_{\mathrm{t}}
\end{gathered}
$$

where $\hat{\mathbf{F}}_{\mathrm{t}}$ is the nodal torque vector, $\hat{\mathbf{K}}_{\mathrm{t}}$ is the spectral stiffness matrix for the torsional rod element, and $\hat{\mathbf{d}}_{\mathrm{t}}$ is the nodal rotation vector. In Figure 2.3 and Eqn (2.52), the shear modulus and torsional stiffness constant of the element are given by $G$ and $J$, respectively.

The added stiffness terms for the out-of-plane bending degrees of freedom are the same as those derived previously in Eqn (2.49) of Section 2.2 except there is now the distinction of rotation in $z-x$ plane occurring in the opposite sense as rotation in the $x-y$ plane. This necessitates a sign change in the appropriate rotation terms of the spectral stiffness matrix in Eqn (2.49). Additionally, there are also two area moment of inertia terms, $I_{y}$ and $I_{z}$, as illustrated in Figure 2.3. The $I_{y}$ area moment of inertia is associated with bending about the local $y$-axis, and $I_{z}$ is associated with bending about the local z-axis.

The increased number of element degrees of freedom results in a larger $12 \times 12$ spectral stiffness matrix for the 3-D space frame element. Despite this, the assembly of multiple elements to form a global structure follows the procedures discussed in 
Section 2.2 with the more involved step of using the three-node method to calculate the direction cosines, and thus, the necessary local-to-global transformation matrix. Post-assembly, solving the system of equations proceeds as described in Section 2.2.

\subsection{Verification Model 1: Semi-Infinite Cantilevered Beam}

The first computational model presented is that of a planar semi-infinite cantilevered beam subject to a transverse impulse load. The purpose of this analysis is to verify the adequacy of the spectral finite element computational code and to compare the results with those from a conventional finite element analysis as done by Doyle \& Farris [18]. Hence, this model serves as a benchmark for the verification of the spectral finite element code. The software program MATLAB was used in generating the code, running the analysis, and visualizing the results. The text by Kwon \& Bang [54] served as a reference in providing an elementary architecture upon which the code was written.

The semi-infinite beam is 85 inches in length, has fixed-free boundary conditions, and is impacted transversely by an impulse load 80 inches from the fixed end. The beam was analyzed using two separate finite element models as shown in Figures 2.4 and 2.5. Table 2.1 gives the physical parameters of the beam. The first model consists of 170 conventional elements each having a length of 0.5 inch. This model has 171 nodes with 513 total degrees of freedom (i.e. 3 degrees of freedom per node). The second model consists of two spectral elements (the first 80 inches in length and the second 5 inches in length) with three nodes and nine degrees of freedom total. The 80 inch element in the spectral model is treated as a throw-off element since it effectively extends to infinity and no wave reflections occur within the timeframe of interest. 


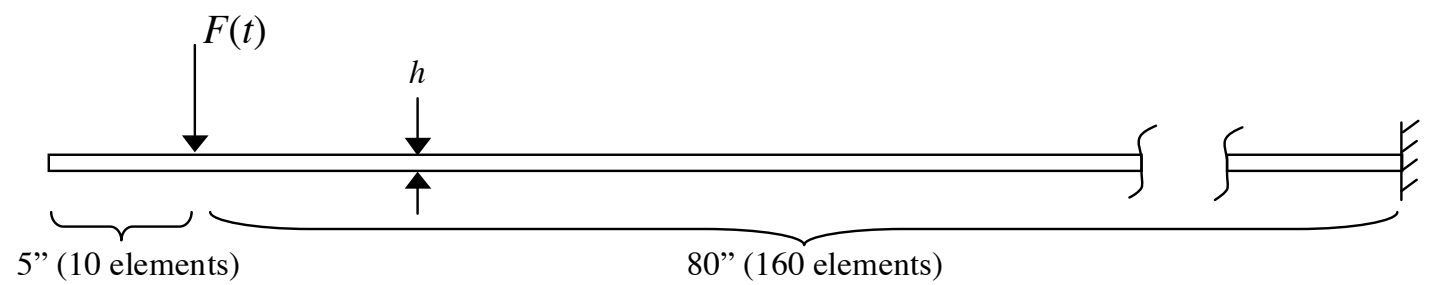

Figure 2.4: Schematic of semi-infinite beam modeled using conventional elements

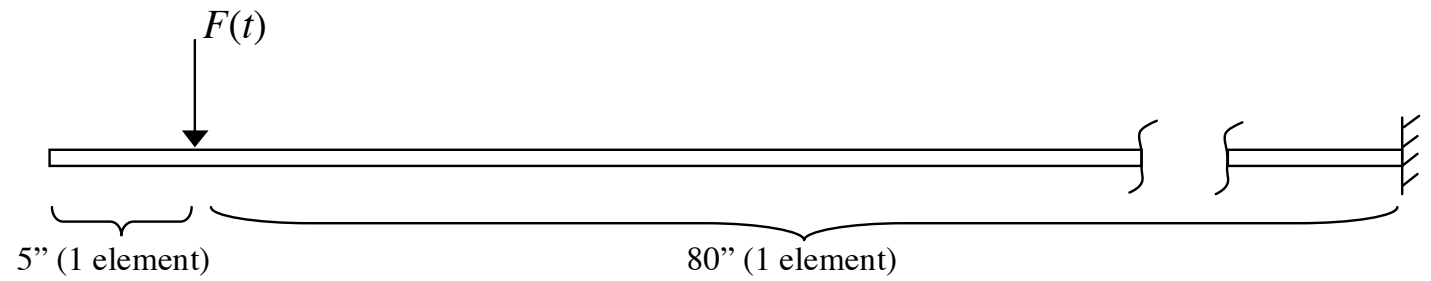

Figure 2.5: Schematic of semi-infinite beam modeled using spectral elements

Table 2.1: Semi-infinite beam physical parameters

\begin{tabular}{cc}
\hline Beam Physical Parameters & Value \\
\hline Beam Width $(h)$ & 0.25 inch \\
Out-of-Plane Beam Depth $(b)$ & 1.00 inch \\
Young's Modulus $(E)$ & $10.6 \times 10^{6} \mathrm{psi}$ \\
Material Density $(\rho)$ & $2.74 \times 10^{4} \mathrm{lb} / \mathrm{in}^{3}$
\end{tabular}




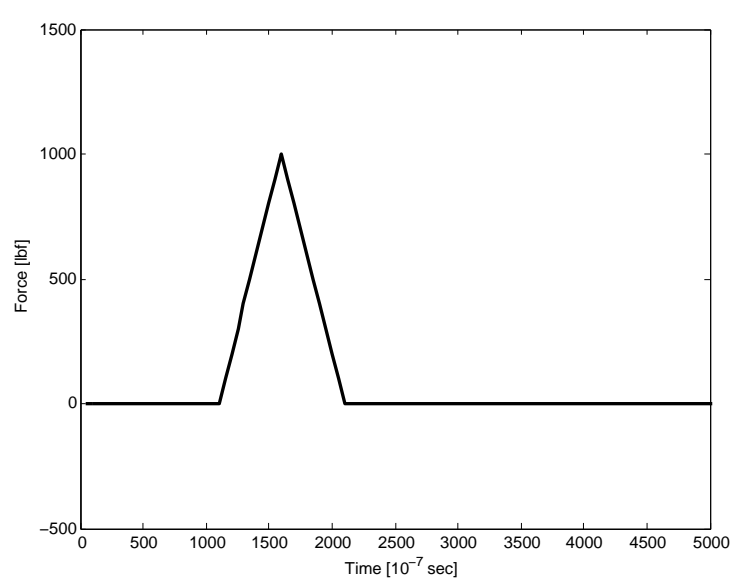

(a) Time history

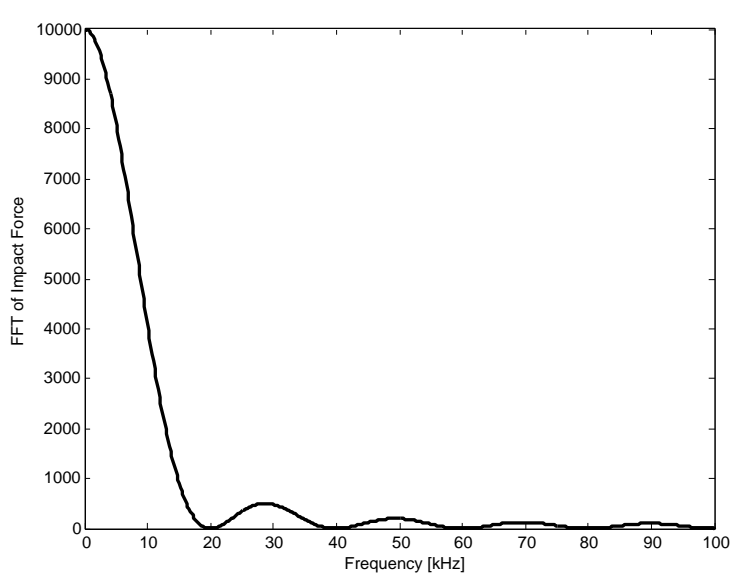

(b) FFT

Figure 2.6: Impulse force: (a) time history and (b) FFT

In analyzing the spectral model, the frequency components of the impulse load were determined by using the Fast Fourier Transfer (FFT) function in MATLAB. A sampling rate of $N=1024$ was used with $\Delta T=5 \mu$ sec. These parameters correspond to a Nyquist (i.e. the $(N / 2+1)^{\text {th }}$ value) frequency $f_{n}=1 /(2 \Delta T)=100 \mathrm{kHz}$. The significance of the Nyquist frequency is that, in order for the FFT to be accurate, the highest noteworthy frequency in the signal must be less than the Nyquist frequency. The time history of the impulse force and the FFT of this time signal are given in Figures 2.6 (a) and (b), respectively. Observe that the frequency components of the pulse are contained well below the Nyquist frequency.

As discussed in Section 2.2, the solution for the spectral element model proceeds by solving the spectral system of equations pseudo-statically over the frequencies of interest. The procedure begins by taking the FFT of the impulse time history to form the spectral forcing vector for the global system at discrete frequencies (reference Figure 2.6). In this numerical example, the frequency steps are $\Delta f=f_{n} /(N / 2)$, or 195.3 Hz. At each frequency step, the spectral stiffness matrix for each element is 


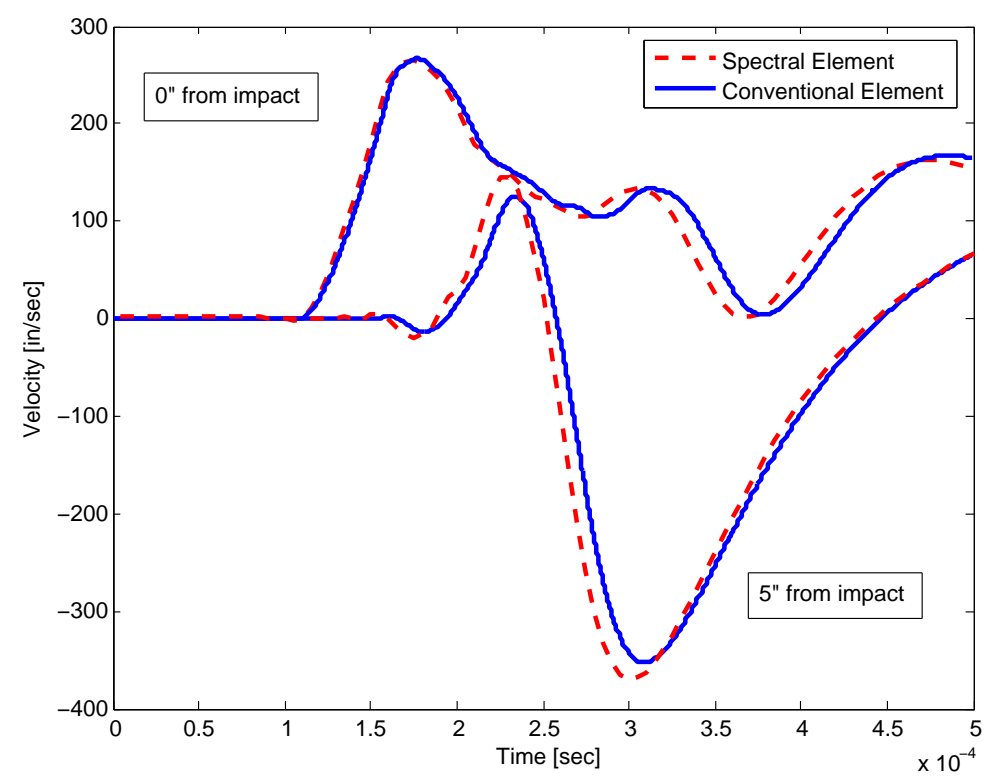

Figure 2.7: Transverse velocity response comparison for semi-infinite beam subject to an impulse load

calculated using the appropriate frequency value, and the global spectral stiffness matrix is assembled in the conventional manner. Next, the displacement boundary conditions are applied to condense both the global force vector and stiffness matrix. Following this, the stiffness matrix is inverted and the spectral displacements are solved for. The spectral displacements are then stored to disk at each frequency step. Once the frequency loop is finished, the time response is reconstructed by taking the inverse FFT of the spectral displacements.

In analyzing the conventional element model, the Newmark method a-form, as outlined in Hughes [42], was used for time integration of the equation of motion. Specifically, the central difference method (i.e. $\beta=0, \gamma=0.5$ ) was used with a time step of $\Delta t=1 \times 10^{-7}$ seconds for a total time of $500 \mu$ sec. Additionally, a lumped mass matrix was used.

In Figure 2.7 a comparison is given of the transverse velocity response of the 
cantilevered beam computed using the conventional finite element method versus the spectral finite element method. The transverse velocity is shown as a function of time at two locations along the beam: 1) the impact site and 2) five inches from the impact site at the free end. The transverse velocity of the beam is a good metric of performance since it best illustrates both the wave propagation phenomenon and the wave reflection phenomenon. Evident in this figure is the remarkable degree of agreement between the results computed using the conventional and spectral element methods. These results also closely match the results from the original work of Doyle $\&$ Farris [18], thus verifying the performance of the computational code. The power of this method is made clear in the reduction of the size of the computational problem from 170 elements to just two. Specifically, for the spectral element solution of this problem, processor time on a standard laptop computer (i.e. $1.33 \mathrm{GHz}$ processor with 768 MB SDRAM memory) running MATLAB is reduced to less than 2 seconds compared with approximately 290 seconds for the conventional element solution.

\subsection{Verification Model 2: Fixed-Fixed Beam}

In this section, a second numerical study is presented for further verification of the capability of the spectral finite element computational code. The transverse and longitudinal natural frequencies of free vibration for a fixed-fixed beam are investigated. Appropriately, this study highlights the ability of the method to predict the propagation of multiple wave types. The effect of mesh density is also examined by modeling the same beam twice using a two spectral element discretization in the first case and a 20 spectral element discretization in the second case. Finally, the results obtained from both computational analyses are compared with the analytical results calculated using the formulas given by Blevins [8]. 
$E, A, I, \rho, L$

Figure 2.8: Schematic of fixed-fixed beam

Table 2.2: Fixed-fixed beam physical parameters

\begin{tabular}{c}
\hline Beam Physical Parameter \\
\hline Beam Width $(h)$ \\
Out-of-Plane Beam Depth $(b)$ \\
Young's Modulus $(E)$ \\
Material Density $(\rho)$ \\
Length $(L)$
\end{tabular}

Value
$0.0011 \mathrm{~m}$
$0.0127 \mathrm{~m}$
$7.1 \times 10^{10} \mathrm{~N} / \mathrm{m}^{2}$
$2700 \mathrm{~kg} / \mathrm{m}^{3}$
$0.4 \mathrm{~m}$

Consider the fixed-fixed beam shown in Figure 2.8. The physical parameters of the beam are given in Table 2.2. Computationally, the resonant frequencies of free vibration for the structure can be found by monitoring the determinant of the global spectral stiffness matrix. Specifically, the determinant of this matrix should approach zero at resonance. Therefore, by computing $\log \left(1 / \operatorname{det}\left[\hat{\mathbf{K}}_{\mathrm{G}}\right]\right)$ over a frequency range of interest, the natural frequencies of the structure can be determined.

The analytical solution for the natural frequencies of a fixed-fixed beam are given by Eqn (2.54) and Eqn (2.55) for bending and axial modes, respectively (see Case \#7 on p. 108 and Case \#3 on p. 184 in Blevins [8]).

$$
\begin{gathered}
f_{q_{\text {_bend }}}=\frac{\lambda_{q}^{2}}{2 \pi L^{2}}\left(\frac{E I}{\rho A}\right)^{1 / 2} \text { where, } \quad \lambda_{q}=(2 q+1) \frac{\pi}{2} \quad ; \text { for } \quad q=1,2,3, \ldots \\
f_{q_{\text {-axial }}}=\frac{\lambda_{q}}{2 \pi L}\left(\frac{E}{\rho}\right)^{1 / 2} \quad \text { where, } \quad \lambda_{q}=q \pi \quad ; \text { for } \quad q=1,2,3, \ldots
\end{gathered}
$$

The finite element analysis was carried out over a frequency range of $0 \mathrm{~Hz}$ to $10 \mathrm{kHz}$ in $1 \mathrm{~Hz}$ frequency steps. Shown in Figure 2.9 are the computed values of $\log \left(1 / \operatorname{det}\left[\hat{\mathbf{K}}_{\mathrm{G}}\right]\right)$ for the two element model over a truncated frequency range of 0 $\mathrm{Hz}$ to $1 \mathrm{kHz}$. The smaller frequency range is used in this figure for visual clarity. 


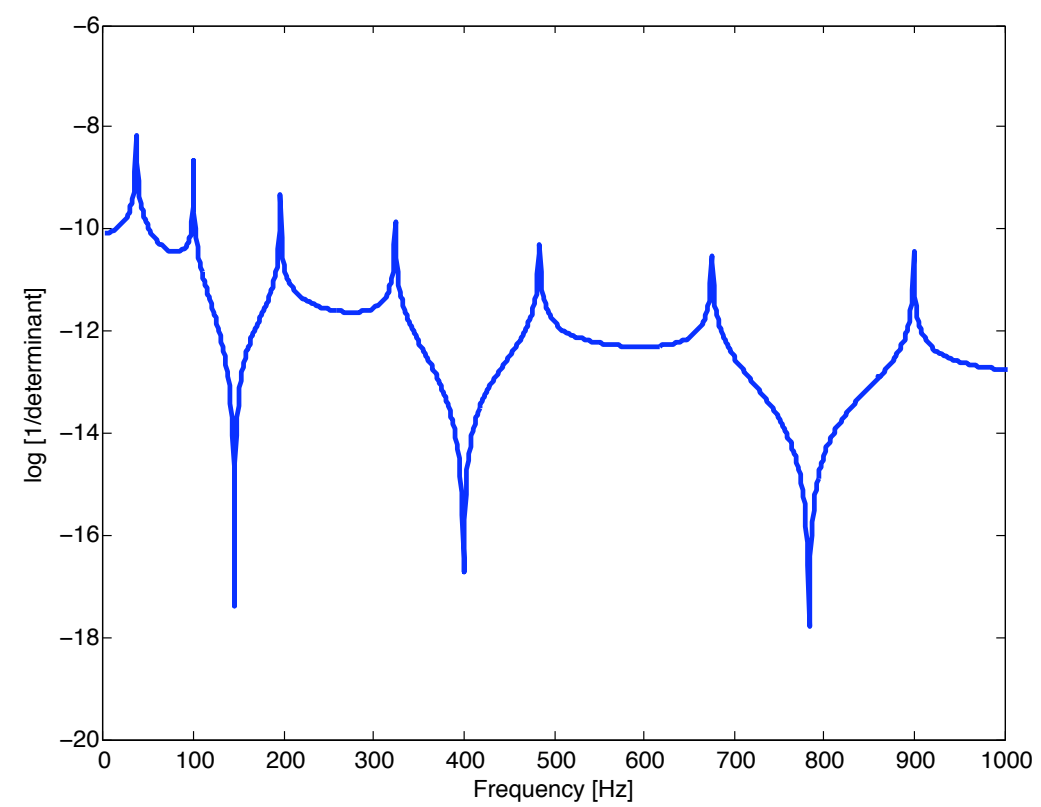

Figure 2.9: Natural frequencies of fixed-fixed beam modeled using two spectral finite elements

The seven peaks visible in Figure 2.9 represent the first seven resonances for the fixed-fixed beam. Thus, using this visualization approach, the natural frequencies were determined for both the two element and 20 element model.

All results are presented over the entire $10 \mathrm{kHz}$ frequency range in the third column of Table 2.3. The analytical values for the natural frequencies of the structure, calculated using Eqns (2.54) and (2.55), are also given in the second column of Table 2.3. Surprisingly, the two element and 20 element computational models predict the exact same natural frequencies, and each computed value is well within $1 \%$ of the corresponding analytical value over the entire frequency range of interest. Moreover, as Table 2.3 shows, the first axial mode is evident at $6410 \mathrm{~Hz}$ in both finite element models. This result underscores how the spectral finite element method is capable of capturing the propagation of different, in this case longitudinal and transverse, waves in a structure. Also, observe that increasing the number of elements from 
two to 20 does not alter the accuracy of the computational results. As previously discussed, this is an artifact of the spectral element formulation, which correctly accounts for wave propagation effects through the assumed element shape function, thus eliminating the need for excessive element discretization.

Table 2.3: Natural frequencies of fixed-fixed beam

\begin{tabular}{cccc}
\hline $\begin{array}{c}\text { Mode number } \\
\text { and type }\end{array}$ & $\begin{array}{c}\text { Natural frequency } \\
\text { calculated using formulas } \\
\text { from Blevins [8] }\end{array}$ & $\begin{array}{c}\text { Natural frequency } \\
\text { computed using both } \\
\text { 2 \& 20 element SFEA }\end{array}$ & $\begin{array}{c}\text { Percent } \\
\text { error }\end{array}$ \\
\hline 1 - Bending & $36.2 \mathrm{~Hz}$ & $36 \mathrm{~Hz}$ & $5.5 \mathrm{E}-1 \%$ \\
2 - Bending & $99.9 \mathrm{~Hz}$ & $100 \mathrm{~Hz}$ & $1.0 \mathrm{E}-1 \%$ \\
3 - Bending & $195.8 \mathrm{~Hz}$ & $196 \mathrm{~Hz}$ & $1.0 \mathrm{E}-1 \%$ \\
4 - Bending & $323.7 \mathrm{~Hz}$ & $324 \mathrm{~Hz}$ & $9.3 \mathrm{E}-2 \%$ \\
5 - Bending & $483.6 \mathrm{~Hz}$ & $484 \mathrm{~Hz}$ & $8.3 \mathrm{E}-2 \%$ \\
6 - Bending & $675.4 \mathrm{~Hz}$ & $675 \mathrm{~Hz}$ & $5.9 \mathrm{E}-2 \%$ \\
7 - Bending & $899.2 \mathrm{~Hz}$ & $899 \mathrm{~Hz}$ & $2.2 \mathrm{E}-2 \%$ \\
8 - Bending & $1155.0 \mathrm{~Hz}$ & $0.0 \%$ \\
9 - Bending & $1442.8 \mathrm{~Hz}$ & $1155 \mathrm{~Hz}$ & $1.4 \mathrm{E}-2 \%$ \\
10 - Bending & $1762.5 \mathrm{~Hz}$ & $1443 \mathrm{~Hz}$ & $2.8 \mathrm{E}-2 \%$ \\
11 - Bending & $2114.2 \mathrm{~Hz}$ & $1762 \mathrm{~Hz}$ & $9.5 \mathrm{E}-3 \%$ \\
12 - Bending & $2497.9 \mathrm{~Hz}$ & $2114 \mathrm{~Hz}$ & $4.0 \mathrm{E}-3 \%$ \\
13 - Bending & $2913.5 \mathrm{~Hz}$ & $2498 \mathrm{~Hz}$ & $1.7 \mathrm{E}-2 \%$ \\
14 - Bending & $3361.1 \mathrm{~Hz}$ & $2914 \mathrm{~Hz}$ & $3.0 \mathrm{E}-3 \%$ \\
15 - Bending & $3840.7 \mathrm{~Hz}$ & $3361 \mathrm{~Hz}$ & $7.8 \mathrm{E}-3 \%$ \\
16 - Bending & $4352.3 \mathrm{~Hz}$ & $3841 \mathrm{~Hz}$ & $6.9 \mathrm{E}-3 \%$ \\
17 - Bending & $4895.8 \mathrm{~Hz}$ & $4352 \mathrm{~Hz}$ & $4.1 \mathrm{E}-3 \%$ \\
18 - Bending & $5471.3 \mathrm{~Hz}$ & $4896 \mathrm{~Hz}$ & $5.5 \mathrm{E}-3 \%$ \\
19 - Bending & $6078.8 \mathrm{~Hz}$ & $5471 \mathrm{~Hz}$ & $3.3 \mathrm{E}-3 \%$ \\
20 - Axial & $6410.0 \mathrm{~Hz}$ & $6079 \mathrm{~Hz}$ & $0.0 \%$ \\
21 - Bending & $6718.3 \mathrm{~Hz}$ & $6410 \mathrm{~Hz}$ & $4.5 \mathrm{E}-3 \%$ \\
22 - Bending & $7389.7 \mathrm{~Hz}$ & $6718 \mathrm{~Hz}$ & $4.1 \mathrm{E}-3 \%$ \\
23 - Bending & $8093.1 \mathrm{~Hz}$ & $7390 \mathrm{~Hz}$ & $1.2 \mathrm{E}-3 \%$ \\
24 - Bending & $8828.5 \mathrm{~Hz}$ & $8093 \mathrm{~Hz}$ & $5.7 \mathrm{E}-3 \%$ \\
25 - Bending & $9595.8 \mathrm{~Hz}$ & $8828 \mathrm{~Hz}$ & $2.1 \mathrm{E}-3 \%$
\end{tabular}




\section{CHAPTER 3}

\section{Analysis \& Design}

In Chapter 2 the computational methods required for the analysis of 2-D and 3-D truss-like structures were presented. It is now possible to focus on the dynamic response analysis of structures having more complicated geometry. The analysis of a square core "unit cell" and a full structure is presented first in Section 3.1; this analysis serves as a benchmark for comparison throughout the remainder of this dissertation. The square core unit cell and structure are subject to an impinging pressure wave on one side, and the radiatory response into a fluid domain is examined on the other side. The structure's output side root mean square (RMS) normal velocity is the figure of merit since it is directly linked to energy of motion, pressure fluctuations, and hence, radiated sound in a fluid domain, per El-Raheb [19], ElRaheb \& Wagner [20], and Ruzzene [84]. The overarching objective of this model is to apply the spectral analysis methodology to a more complicated problem where the mid-frequency vibro-acoustic response of a structure is of interest.

The concepts and definitions associated with the analysis and design of a compliant mechanism unit cell and structure are introduced in Section 3.2. This section contains the seminal study of this research. Both the unit cell and structure are subject to an incident pressure wave on one side while the normal velocity response is computed on the other side. The numerical results from this analysis are compared with those obtained from the analysis of the square core unit cell and structure. A 
determination is made as to whether or not velocity attenuation is achieved across a prescribed frequency range. Following this, a second compliant mechanism topology is developed and analyzed in order to independently verify the concepts proposed via the first model.

These initial studies are built upon through further investigation into the design of structures made from alternative (i.e. nonmetallic) materials in Section 3.3. A moldable polymer structure is used to illustrate the fundamental relationship between the material a structure is built from and the unit cell sizing.

In Section 3.4 the dynamic behavior of parallel and serial connected structures is outlined. The standalone and additive composite effects of stacking and coupling compliant mechanism layers having separate response characteristics is addressed. The results in this section are directed towards answering the question of how to further minimize structural dynamic response. Additionally, this section provides an introduction to the design of larger multi-functional structures.

Lastly, in Section 3.5 the analysis and design of a 3-D compliant mechanism unit cell and lattice panel serves to demonstrate how the principles upon which 2-D structures are based can be extended to the design of structures having more realistic spatial geometry.

\subsection{Benchmark Square Core Unit Cell \& Structure}

The numerical study in this section applies the spectral finite element method to the analysis of a square core unit cell, Figure 3.1, and a structure consisting of an assemblage of 20 periodic square core unit cells, Figure 3.2. The structure is treated as a truss beam connected to a rigid baffle at both ends and subjected to a distributed load induced by an incident pressure wave. 


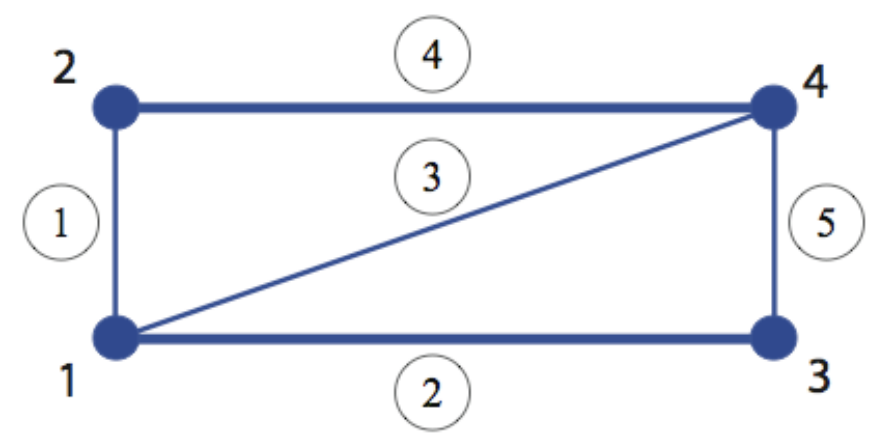

Figure 3.1: Square core unit cell (numbers that are circled denote elements, numbers that are not circled denote nodes)

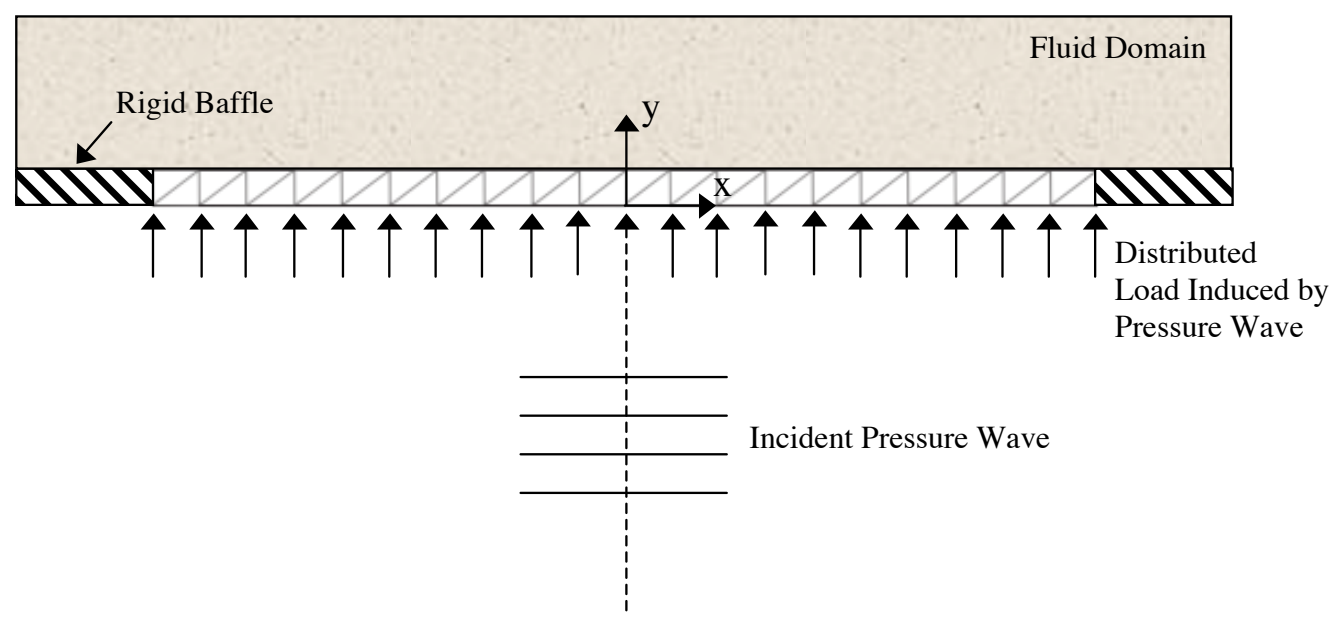

Figure 3.2: Periodic square core structure

\subsubsection{Analysis}

As shown in Figure 3.2, the bottom layer of the truss beam is excited by an incident pressure wave front. Prior to solving for the response of the unit cell or structure, the spectral load vector equivalent to the incident pressure for each bottom layer element must be formulated. In two dimensions, the standard form for the harmonic variation of pressure, $p_{\mathrm{i}}$, in time is

$$
p_{\mathrm{i}}(x, y, t)=p_{\mathrm{i}}(x, y, \omega) \mathrm{e}^{-\mathrm{i} \omega t}
$$


Assuming an incident pressure wave that impinges perpendicular to the bottom layer of the structure, the pressure in the frequency domain is written as

$$
p_{\mathrm{i}}(x, y, \omega)=p_{\text {in }}
$$

where $p_{\text {in }}$ is the amplitude of the normally incident pressure wave.

Using this description of the pressure, the spectral work done by the impinging wave on a bottom layer element of the structure can be defined at a specific frequency as

$$
\hat{W}_{e}(\omega)=\int_{0}^{L_{e}} p_{\text {in }} \hat{v}_{\text {bot }}(x, \omega) b d x
$$

where $b$ is the out-of-plane element depth, and $\hat{v}_{\text {bot }}$ is the transverse spectral displacement of the bottom layer element. Substituting the coefficients obtained from Eqn (2.42) into Eqn (2.36), the transverse spectral displacement is written in terms of the vector of spectral beam element nodal shape functions, $\hat{\mathbf{N}}_{\mathrm{b}}$, as

$$
\hat{v}_{\text {bot }}(x, \omega)=\hat{\mathbf{d}}_{\mathrm{b}}^{\mathrm{T}} \hat{\mathbf{N}}_{\mathrm{b}}(x, \omega)
$$

Combining Eqn (3.3) with Eqn (3.4), the spectral work takes the following form

$$
\hat{W}_{e}(\omega)=\hat{\mathbf{d}}_{\mathrm{b}}^{\mathrm{T}} \int_{0}^{L_{e}} p_{\mathrm{in}} \hat{\mathbf{N}}_{\mathrm{b}}(x, \omega) b d x
$$

Or,

$$
\hat{W}_{e}(\omega)=\hat{\mathbf{d}}_{\mathrm{b}}^{\mathrm{T}} \hat{\mathbf{F}}_{e}(\omega)
$$

where the spectral load vector, $\hat{\mathbf{F}}_{e}$, is

$$
\hat{\mathbf{F}}_{e}(\omega)=\int_{0}^{L_{e}} p_{\text {in }} \hat{\mathbf{N}}_{\mathrm{b}}(x, \omega) b d x
$$

Or, similarly

$$
\hat{\mathbf{F}}_{e}(\omega)=\int_{0}^{L_{e}} p_{\text {in }} \hat{\mathbf{w}}_{\mathrm{b}}(x, \omega) d x
$$


where $\hat{\mathbf{w}}_{\mathrm{b}}$ is the spectral weighting function for the beam element.

Symmetric boundary conditions, representative of those imposed on a unit cell when assembled into the full structure, are enforced in the unit cell analysis. Rotational degrees of freedom of the nodes on the left and right edges of the unit cell are constrained with all remaining degrees of freedom unconstrained. For the full structure, only the degrees of freedom of nodes interfacing with the rigid baffles are constrained. Unless otherwise stated, the forcing function and boundary conditions for the unit cell and structure are implemented in the exact manner described above for all of the remaining computational analyses in this dissertation.

Following El-Raheb \& Wagner [20], the structural dynamic response is computed in terms of the top layer RMS transverse velocity as

$$
\mathrm{V}_{\mathrm{RMS}}=20 \log _{10}\left[\frac{\omega}{\mathrm{V}_{\mathrm{ref}}}\left[\frac{1}{N} \sum_{j=1}^{N}\left|\hat{v}_{j}^{\text {top }}\right|^{2}\right]^{1 / 2}\right]
$$

where $\omega$ is the frequency of interest, $\mathrm{v}_{\text {ref }}$ is a reference velocity, $\hat{v}^{\text {top }}$ is the transverse spectral displacement of the $j^{\text {th }}$ node of interest, and $N$ is the number of top layer nodes. In the next section, the RMS normal velocity response of the square core unit cell and structure are given and compared with existing results from the research of El-Raheb [19], El-Raheb \& Wagner [20], and Ruzzene [84].

\subsubsection{Computational Results}

Each new unit cell and structure that is introduced in this dissertation is assumed to be made of aluminum material unless specified otherwise. This material is taken to have a Young's modulus, $E=7.1 \times 10^{10} \mathrm{~N} / \mathrm{m}^{2}$, and density, $\rho=2700 \mathrm{~kg} / \mathrm{m}^{3}$. As is typical of such structures, hysteretic material damping is introduced to account for inherent energy loss. A complex modulus, $E^{*}=E(1+\mathrm{i} \eta)$, is utilized, where the loss factor, $\eta=0.01$. This value is slightly high relative to typical loss factor values 
reported for aluminum by Lazan [58], but it is consistent with prior research by ElRaheb \& Wagner [20] and Ruzzene [84]. Nonetheless, the effect of using a slightly higher loss factor has been explored with the result being that resonant peaks are damped, whereas the fundamental frequency response of general interest is basically unchanged.

Unit cell dimensions of $0.1 \mathrm{~m}$ by $0.05 \mathrm{~m}$, and structure dimensions of $2 \mathrm{~m}$ by 0.05 $\mathrm{m}$, are used. The out-of-plane element depth, $b$, for all elements is set to unity. With noted exceptions, this is the standard out-of-plane depth for all two-dimensional structures described in this work. The width of the "core" elements (i.e. elements 1 , 3, and 5 in Figure 3.1) of the unit cell is $2.5 \mathrm{~mm}$ while the top and bottom facesheet thickness (i.e. elements 2 and 4 in Figure 3.1) is taken as $5.0 \mathrm{~mm}$.

In the analysis of the unit cell and structure the incident pressure field amplitude in Eqn (3.8) is $p_{\text {in }}=1 \mathrm{~N} / \mathrm{m}^{2}$. The RMS transverse velocity of the unit cell and structure is computed using a reference velocity, $\mathrm{v}_{\mathrm{ref}}=10^{-8} \mathrm{~m} / \mathrm{s}$, in Eqn (3.9). These values represent the standard convention for the incident pressure field amplitude and reference velocity used throughout this dissertation.

The frequency range of interest for this study is $0 \mathrm{~Hz}$ to $6000 \mathrm{~Hz}$, evaluated at 10 $\mathrm{Hz}$ frequency steps. The RMS normal velocity responses for the square core unit cell and the structure are given in Figures 3.3 (a) and (b), respectively. Four resonances of the unit cell are present at $1120 \mathrm{~Hz}, 2600 \mathrm{~Hz}, 3700 \mathrm{~Hz}$ and $5910 \mathrm{~Hz}$. Above $2 \mathrm{kHz}$ response is attenuated at three frequencies: 1) at $2660 \mathrm{~Hz}$ just after the resonance at $2600 \mathrm{~Hz}, 2)$ at $4600 \mathrm{~Hz}$ following the resonance at $3700 \mathrm{~Hz}$, and 3) at $5740 \mathrm{~Hz}$ just prior to the resonance at $5910 \mathrm{~Hz}$.

Observe that the unit cell response predicts the structure response, particularly at higher frequencies, where the deformation of the structure is dominated by lo- 


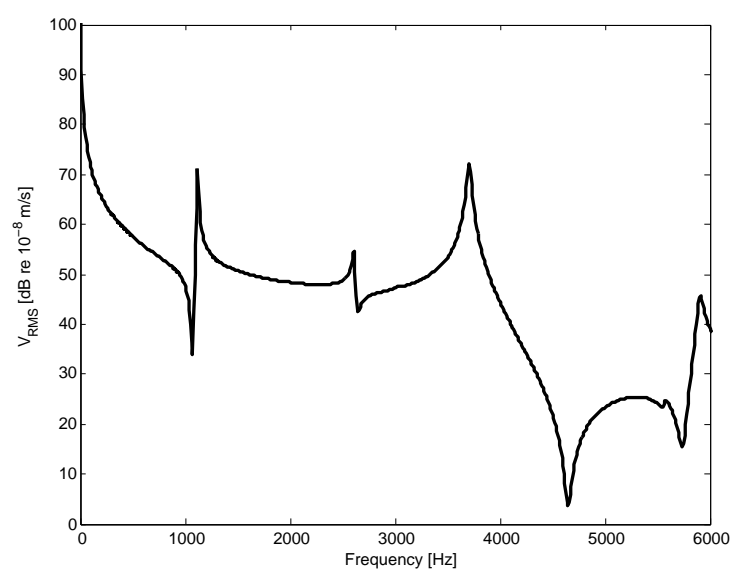

(a) Unit cell

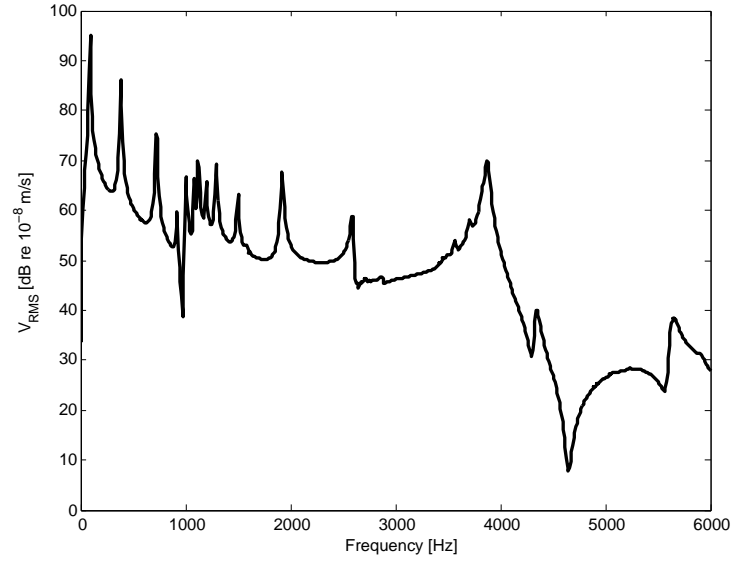

(b) Structure

Figure 3.3: Top layer RMS velocity of square core structure: (a) unit cell and (b) structure

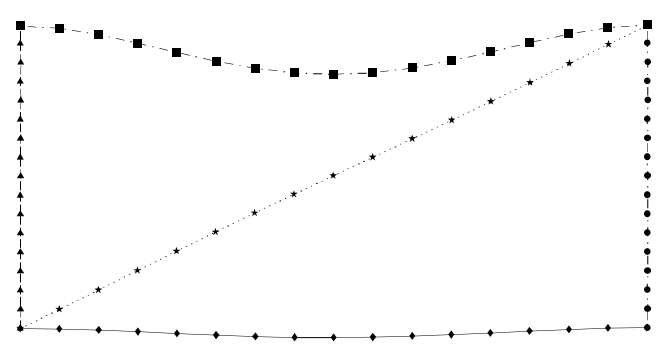

(a) $2600 \mathrm{~Hz}$

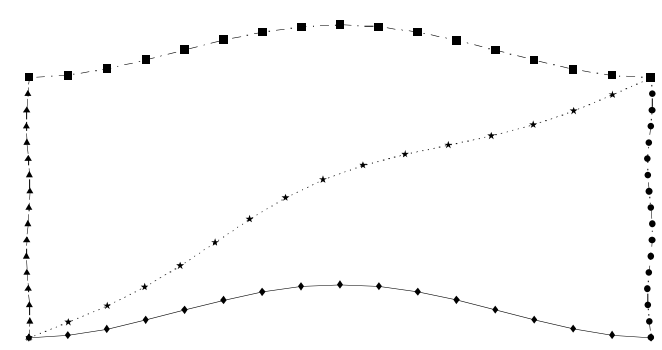

(c) $3700 \mathrm{~Hz}$

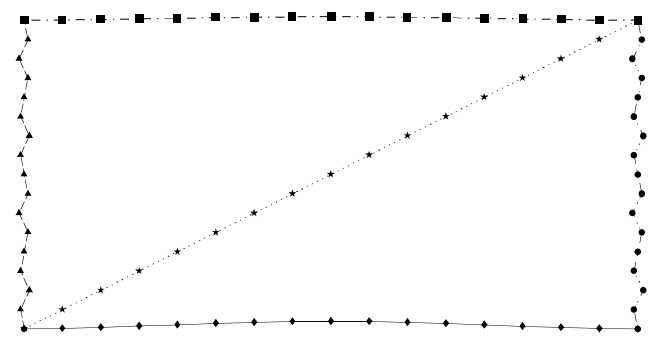

(b) $2660 \mathrm{~Hz}$

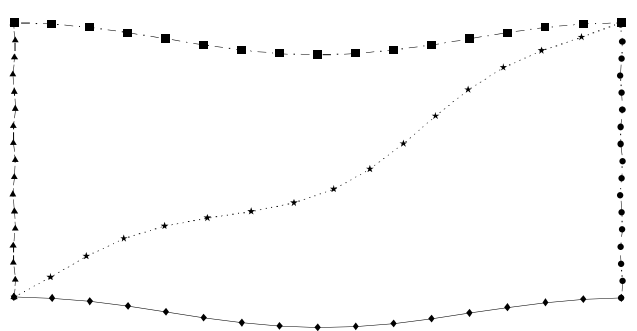

(d) $4600 \mathrm{~Hz}$

Figure 3.4: Deformed shapes of square core unit cell: (a) $2600 \mathrm{~Hz}$, (b) $2660 \mathrm{~Hz}$, (c) $3700 \mathrm{~Hz}$, and (d) $4600 \mathrm{~Hz}$ 


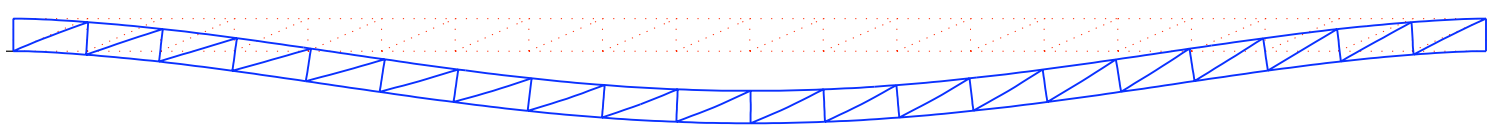

(a) $90 \mathrm{~Hz}$

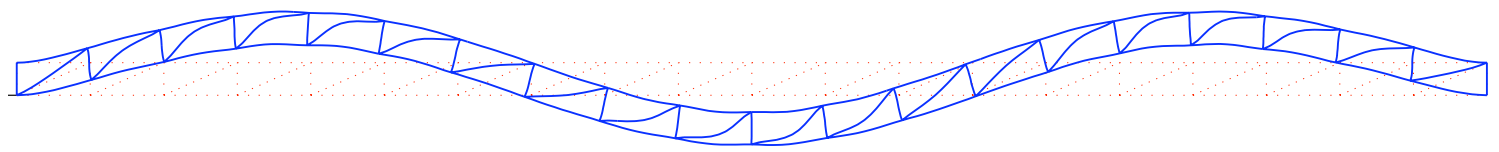

(b) $380 \mathrm{~Hz}$

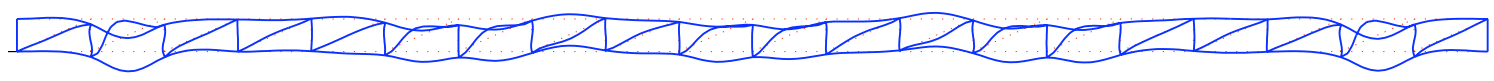

(c) $1500 \mathrm{~Hz}$

Figure 3.5: Deformed shapes of square core structure: (a) $90 \mathrm{~Hz}$, (b) $380 \mathrm{~Hz}$, and (c) $1500 \mathrm{~Hz}$ (dotted lines denote undeformed configuration, continuous lines denote deformed configuration)

cal deformations of the unit cell. The deformed shapes of the unit cell shown at various frequencies in Figure 3.4 illustrate this phenomenon. The peaks in RMS normal velocity response at $2600 \mathrm{~Hz}$ and $3700 \mathrm{~Hz}$ correspond to deflection of the top and bottom layers, with strong coupling between the two. On the other hand, the deformed shapes at $2660 \mathrm{~Hz}$ and $4600 \mathrm{~Hz}$ correspond to greater deflection of the core members. This suggests that at specific frequencies the vibratory energy either excites the core or bypasses it. This result is similar to Ruzzene's work [84], except for the deformed shape at $2660 \mathrm{~Hz}$. While Ruzzene computed deflections of the diagonal core member, the response from this analysis predicts deflections of the vertical core members instead. Despite this, the peak in response at $3700 \mathrm{~Hz}$ does match, demonstrating the largest deflection of the top and bottom members and 
corresponding to the greatest top layer RMS normal velocity response above $2 \mathrm{kHz}$.

The transition from a global level response to a local level response is further explained through the deformed shapes of the full structure given in Figure 3.5. The appearance of a greater number of waves propagating through the structure is visible at increased frequencies, which is consistent with the response described by El-Raheb [19] and El-Raheb \& Wagner [20].

The exploitation of the local level deformations of a structure at middle frequencies is the foundation for the concepts outlined in the remainder of this dissertation. Specifically, in the next section the concept of designing the unit cell core in order to prevent energy transmission is introduced.

\subsection{Compliant Mechanism Unit Cell \& Structure}

The results presented in Section 3.1.2 demonstrate the suitability of using a local level unit cell analysis in predicting the global response of periodic structures like the one shown in Figure 3.2. This section builds upon these results by establishing that compliant mechanisms can be used effectively at a local unit cell level to attenuate broadband vibro-acoustic response at a global level. Specifically, the unit cell can be thought of as a framework into which a compliant mechanism topology is placed in order to employ local level deformations and reduce the output normal velocity of a structure.

\subsubsection{Concept \& Definitions: Compliant Mechanism 1}

The notion of transmitted sound being related to the normal velocity of a radiating object suggests the use of amplification principles in reducing the output (i.e. top layer) normal velocity of a unit cell or structure. Specifically, the principle

of mechanical advantage is explored in the synthesis of an appropriate compliant 
mechanism topology.

Generally, a compliant mechanism topology with a large mechanical advantage is sought. Let $\mathbf{F}_{\text {in }}$ and $\mathbf{F}_{\text {out }}$ be the respective input and output force vectors; similarly $\mathbf{v}_{\text {in }}$ and $\mathbf{v}_{\text {out }}$ denote the velocity vectors of the points of force input and output, respectively. Then, assuming power conservation

$$
P_{\text {out }}=P_{\text {in }} \quad \Rightarrow \quad \mathbf{F}_{\text {out }}^{\mathrm{T}} \mathbf{v}_{\text {out }}=\mathbf{F}_{\text {in }}^{\mathrm{T}} \mathbf{v}_{\text {in }}
$$

where $P_{\text {out }}$ is the mechanism output power and $P_{\text {in }}$ is the mechanism input power. Assuming that the velocity and force vectors are parallel, mechanical advantage is then defined by Erdman et al. [22] as

$$
\mathrm{MA}=\frac{F_{\text {out }}}{F_{\text {in }}}
$$

where the scalar quantities, $F_{\text {out }}$ and $F_{\text {in }}$, denote the corresponding force vector magnitudes and MA is the mechanical advantage.

Eqn (3.11) provides the mechanical advantage for a rigid body mechanism in which power is conserved. However, as Salamon \& Midha note [87], for a compliant mechanism power is ultimately not conserved since, when loaded, elastic energy is stored due to member flexibility. They go on to show that the actual mechanical advantage is really some fraction of the rigid body value expressed as

$$
\mathrm{MA}=\frac{F_{\text {out }}}{F_{\text {in }}}\left(1-\frac{F_{\text {compliant }}}{F_{\text {in }}}\right)
$$

where $F_{\text {compliant }}$ is the part of the input force required to elastically deform the mechanism members.

Notwithstanding this more thorough treatment of mechanical advantage, from this point forward, $F_{\text {compliant }}$ is neglected. This is an assumption used to simplify the process of selecting a mechanism topology. Thus, Eqn (3.12) is reduced back to 


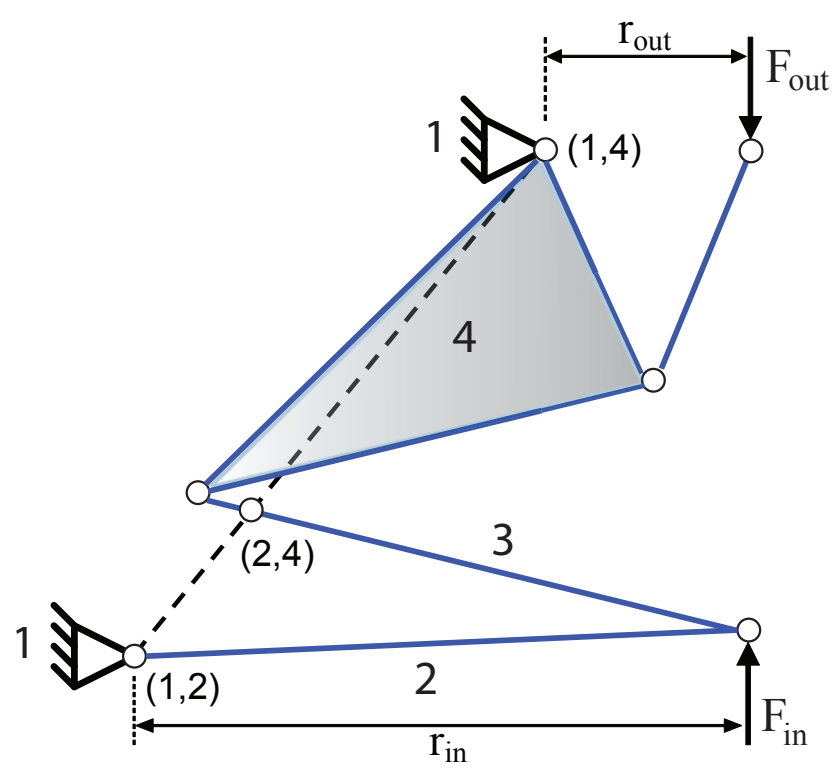

Figure 3.6: Rigid link 4-bar mechanism diagram (numbers denote links, $(\cdot, \cdot)$ denote instant centers)

Eqn (3.11), and using the magnitudes of the respective velocity vectors the MA is written as

$$
\mathrm{MA}=\frac{\mathrm{v}_{\mathrm{in}}}{\mathrm{v}_{\text {out }}}
$$

The significance of Eqn (3.13) is that for a given input speed, $\mathrm{v}_{\text {in }}$, the output speed, $\mathrm{v}_{\text {out }}$, can be reduced by seeking a mechanism topology with a large mechanical advantage.

Based on this conclusion, the compliant stroke amplifier developed by Hetrick \& Kota [38] and Kota et al. [53] (introduced in Section 1.2.4) was chosen as the first compliant mechanism topology for investigation. A prototypical rigid link version of this 4-bar mechanism is shown in Figure 3.6 with a compliant mechanism unit cell realization of the rigid link mechanism given in Figure 3.7. The equation for calculating the mechanical advantage, $\mathrm{MA}_{\mathrm{CM} 1}$, of this rigid link mechanism is

$$
\mathrm{MA}_{\mathrm{CM} 1}=\frac{\mathrm{v}_{\mathrm{in}}}{\mathrm{v}_{\mathrm{out}}}=\frac{r_{\mathrm{in}}}{r_{\mathrm{out}}} \frac{((1,4)-(2,4))}{((1,2)-(2,4))}
$$




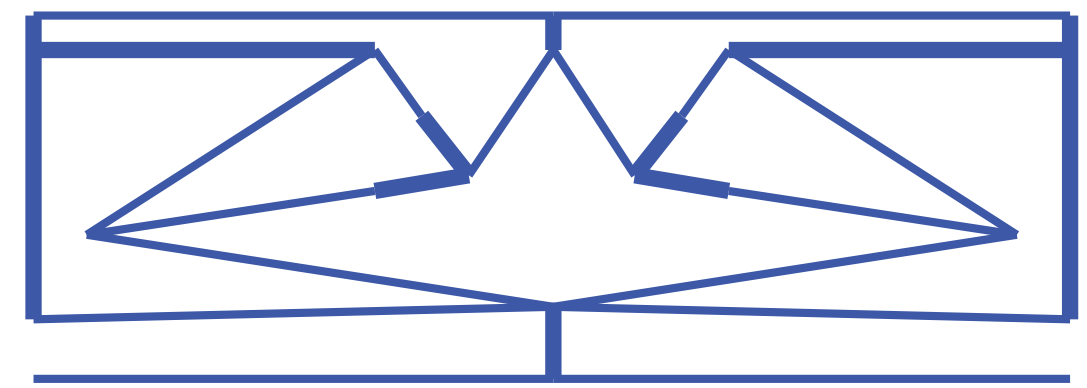

Figure 3.7: Compliant mechanism unit cell model

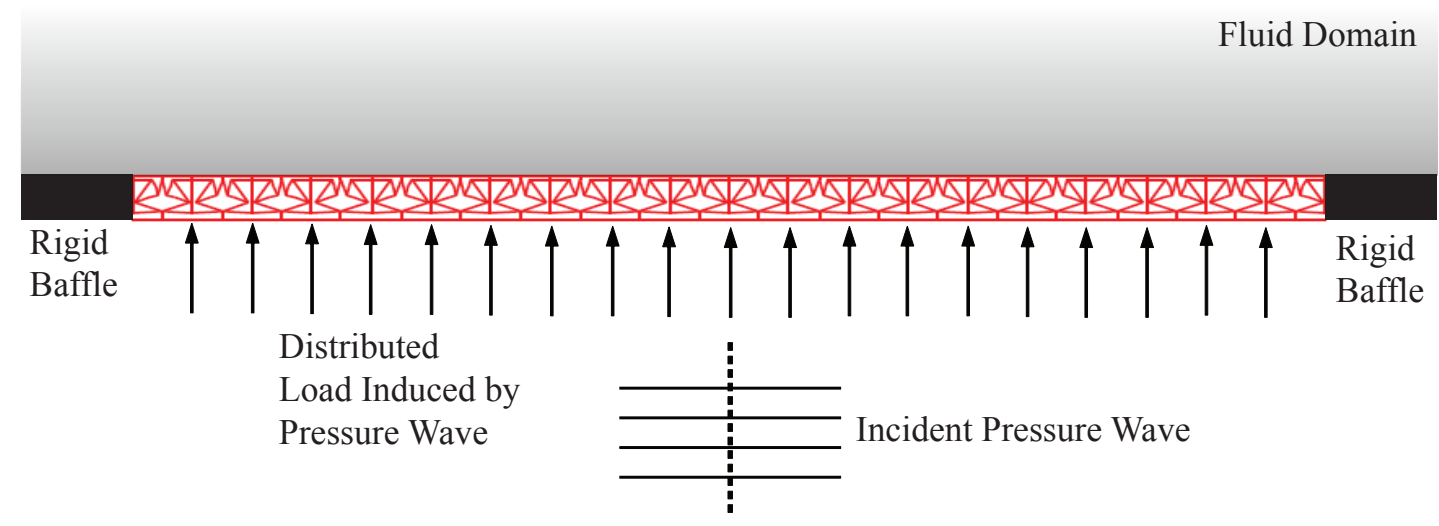

Figure 3.8: Periodic compliant mechanism structure

where $r_{\text {in }}$ is the mechanism input radius, $r_{\text {out }}$ is the mechanism output radius, $(\cdot, \cdot)$ represents an instant center between two links, and $((\cdot, \cdot)-(\cdot, \cdot))$ represents the distance between two respective instant centers, per Erdman et al. [22].

The conceptual model of a full compliant mechanism structure is shown in Figure 3.8, comprising an assembly of 20 unit cells. The structure is again fixed to a rigid baffle at both ends and is subjected to a distributed load induced by an incident pressure wave.

In the compliant mechanism unit cell model given in Figure 3.9, frame elements 

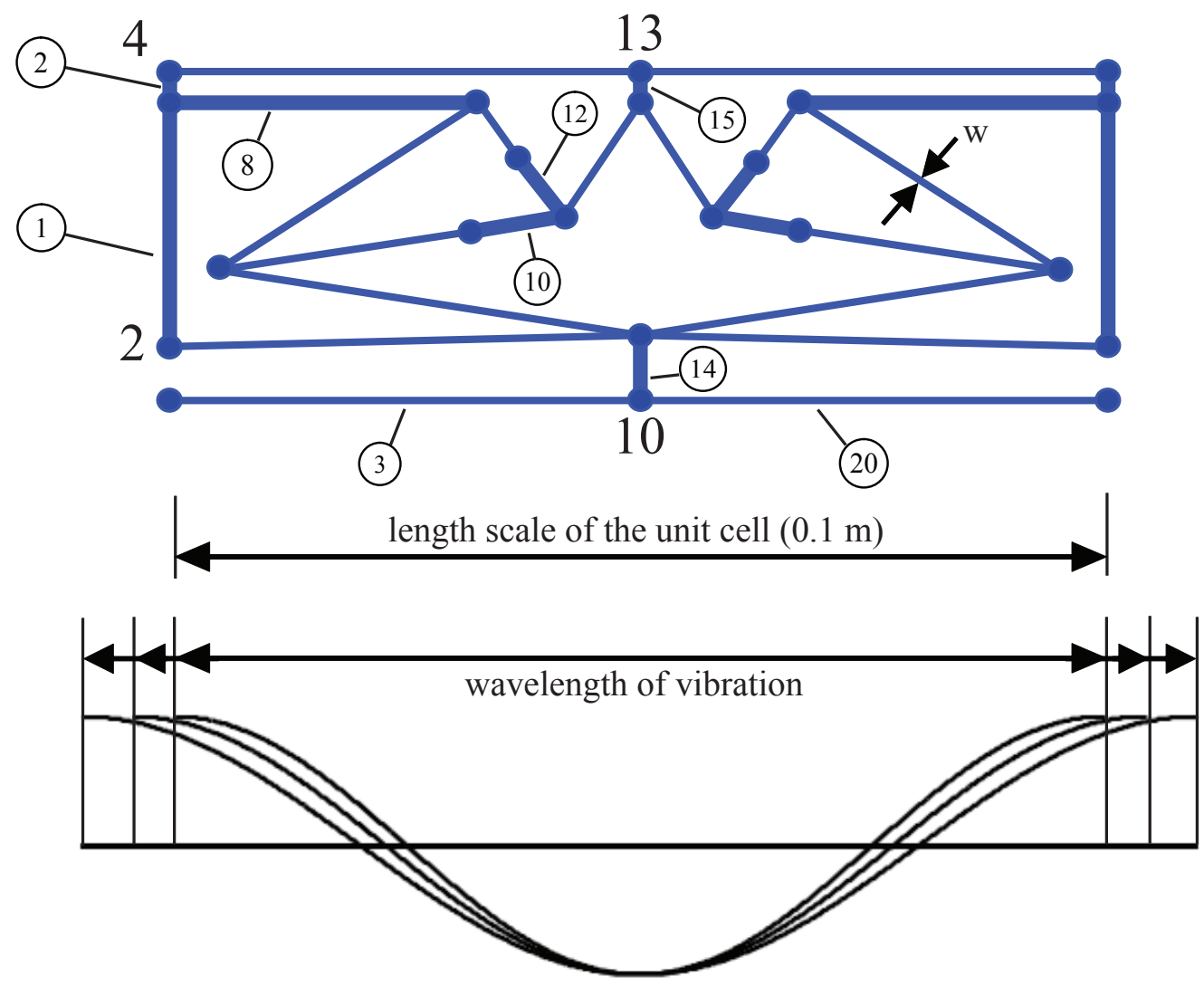

Figure 3.9: Compliant mechanism unit cell model (numbers that are circled denote elements, numbers that are not circled denote nodes)

$1,2,8,10,12,14,15$ and their symmetric counterparts are shown with thicker lines. This implies that these elements are modeled as stiffer beams than the rest of the unit cell although they still technically have compliance. The purpose of making elements 1, 2, and 8 stiffer is that these elements represent the "ground" for the mechanism. Elements 10 and 12 provide reinforcement for link 4 as shown in the rigid link mechanism diagram. Lastly, the input to the mechanism, element 14, and its output counterpart, element 15, are also modeled with stiffer beams since they are "transmission" members and minimal deflection is desired. The remaining elements in the unit cell are considered core members in which greater flexibility is sought.

In terms of element connectivity within the unit cell, the ground elements are 
connected to the output/top side of the structure. The mechanism input is then connected through a single element to the bottom layer allowing transverse waves generated by the incident pressure field to propagate through elements 3 and 20 . As the wavelength of vibration, $\lambda$, approaches the length scale of the unit cell (Figure 3.9), these transverse waves actuate the compliant mechanism evoking mechanical advantage and reducing the top layer normal velocity of the structure.

While the unit cell element connectivity affects the frequency at which response attenuation starts, the width, $w$ in Figure 3.9, of the core elements in the unit cell dictates the attenuation zone cutoff. The natural frequency of the mechanism is linked to this sizing, with increased stiffness leading to a greater cutoff frequency. The frequency response function (FRF) amplitude and phase of the mechanism output displacement relative to input displacement are defined to illustrate this relationship

$$
\text { Amplitude }=\left|\frac{\hat{v}_{13}-\hat{v}_{4}}{\hat{v}_{10}-\hat{v}_{2}}\right| \quad ; \quad \text { Phase }=\frac{180}{\pi}\left[\angle\left(\hat{v}_{13}-\hat{v}_{4}\right)-\angle\left(\hat{v}_{10}-\hat{v}_{2}\right)\right]
$$

where the output is taken as the transverse spectral displacement of node 13 relative to node 4 , while the input is taken as the transverse spectral displacement of node 10 relative to node 2 . Nodes 4 and 2 are selected since they are part of the ground of the mechanism.

Lastly, to predict the frequency at which attenuation begins a straightforward wavenumber analysis is used. The wavenumber for transverse waves in a beam element, from Eqn (2.36), and its relationship to wavelength are combined to yield a wavenumber-frequency equation

$$
k=\sqrt{\omega}\left[\frac{\rho A}{E I}\right]^{1 / 4} \quad \text { and, } \quad \lambda=\frac{2 \pi}{k} \quad \Rightarrow \quad \omega=\left(\frac{2 \pi}{\lambda}\right)^{2} \sqrt{\frac{E I}{\rho A}}
$$

where $\rho, E, A$, and $I$ are the physical parameters of the bottom layer elements. 


\subsubsection{Analysis: Compliant Mechanism 1}

While the top layer RMS normal velocity of the structure, found using Eqn (3.9), is related to the energy of motion, another measure of the reduction of sound radiated by the compliant mechanism structure is the far-field sound pressure level (SPL). A numerical approximation to the SPL for a baffled rectangular radiator was suggested by Ruzzene [84]. Specifically, the pressure level distribution in the acoustic fluid domain above the structure is evaluated numerically using the results from the spectral finite element analysis by way of the Fourier transform solution to the Helmholtz equation.

$$
p_{\mathrm{t}}(x, y, \omega) \cong \frac{-\mathrm{i} \rho_{f} \omega^{2}}{2 \pi} \sum_{-m}^{m} \hat{v}_{\mathrm{t}}\left(m \Delta \gamma_{x}, \omega\right) \frac{\mathrm{e}^{\mathrm{i} y} \sqrt{k^{2}-\left(m \Delta \gamma_{x}\right)^{2}}}{\sqrt{k^{2}-\left(m \Delta \gamma_{x}\right)^{2}}} \mathrm{e}^{\mathrm{i} m \Delta \gamma_{x} x} \Delta \gamma_{x}
$$

This equation is the numerical form of the integral representation of the pressure at a specific frequency, $\omega$, of interest. The integral is approximated as a summation over, $m$, number of finite, $\Delta \gamma_{x}$, steps; where, $\gamma_{x}$ is the usual transform parameter, per Junger and Feit [49]. In Eqn (3.17) $\rho_{f}$ is the density of the fluid and $k=\omega / c_{a}$ is the acoustic wavenumber, where $c_{a}$ is the speed of sound in the fluid. The form of

the numerically evaluated transverse displacement transform, $\hat{v}_{\mathrm{t}}\left(m \Delta \gamma_{x}, \omega\right)$, is given by Ruzzene [84].

In the next section, the RMS normal velocity response and the far-field SPL distribution of the compliant mechanism unit cell and structure are compared, respectively, with that of the square core unit cell and structure from Section 3.1.2.

\subsubsection{Computational Results: Compliant Mechanism 1}

Unit cell dimensions of $0.1 \mathrm{~m}$ by $0.05 \mathrm{~m}$, and structure dimensions of $2.0 \mathrm{~m}$ by 0.05 $\mathrm{m}$, are used. The sizing of the unit cell leads to a compliant mechanism mechanical advantage that is calculated using Eqn (3.14) as 8.3. The width of core elements 
for the compliant mechanism unit cell are taken as $2.5 \mathrm{~mm}$ while stiffer members (e.g. ground, transmission, etc.) are given a width of $5.0 \mathrm{~mm}$. These widths were chosen since they are comparable to those used in the square core structure, which had a core member thickness of $2.5 \mathrm{~mm}$ and facesheet thickness of $5.0 \mathrm{~mm}$. However, despite this comparable sizing, the total mass of the compliant mechanism unit cell is 1.61 times that of the square core unit cell due to a greater number of core members.

The frequency range of interest for this study is $0 \mathrm{~Hz}$ to $6000 \mathrm{~Hz}$, evaluated at $10 \mathrm{~Hz}$ frequency steps. The unit cell FRF is given in Figure 3.10 where the amplitude and phase are scaled for visual clarity by factors of 10 and $1 / 10$, respectively. Below $2 \mathrm{kHz}$ two prominent peaks in response are visible at $880 \mathrm{~Hz}$ and $1780 \mathrm{~Hz}$ representing resonances of the unit cell at frequencies below the attenuation zone starting frequency. Substituting the physical parameters of elements 3 and 20 into Eqn (3.16), along with $\lambda=0.1 \mathrm{~m}$ (i.e. the length of the unit cell), gives an attenuation starting frequency of $2320 \mathrm{~Hz}$, accurately predicting the response shown in Figure 3.10. Moreover, the FRF illustrates that within the attenuation zone the compliant mechanism exhibits non-resonant (i.e. small amplitude), out-of-phase "inversor" behavior. At the cutoff frequency of $5300 \mathrm{~Hz}$ resonance is characterized by a large amplitude and in-phase behavior.

The top layer RMS transverse velocity of the compliant mechanism unit cell and structure are graphed in comparison with the square core unit cell and structure, respectively in Figures 3.11 and 3.12. For the compliant mechanism structure, the resonances at $880 \mathrm{~Hz}$ and $1780 \mathrm{~Hz}$, visible in the unit cell response, are present as expected. Between $2320 \mathrm{~Hz}$ and $5300 \mathrm{~Hz}$ significant broadband attenuation is apparent, most notably at $3700 \mathrm{~Hz}$, where an average reduction of approximately 40 $\mathrm{dB}$ is visible relative to the square core structure. 


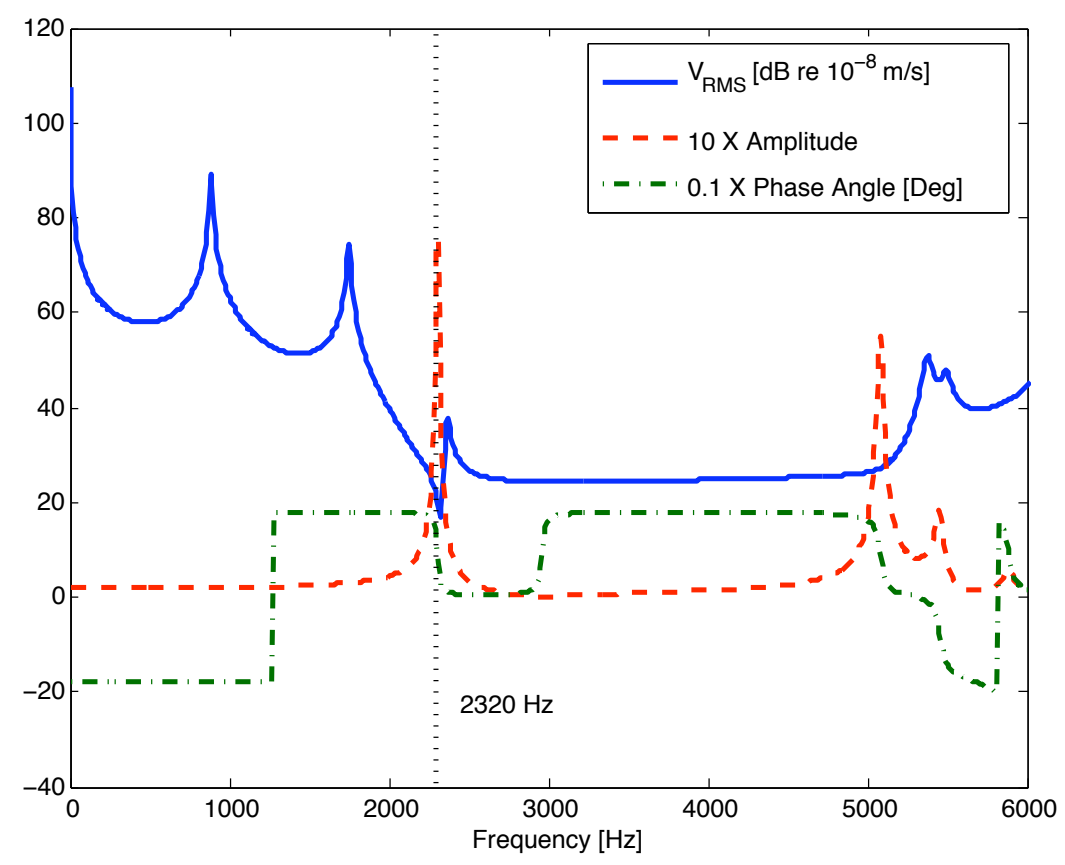

Figure 3.10: Compliant mechanism unit cell FRF

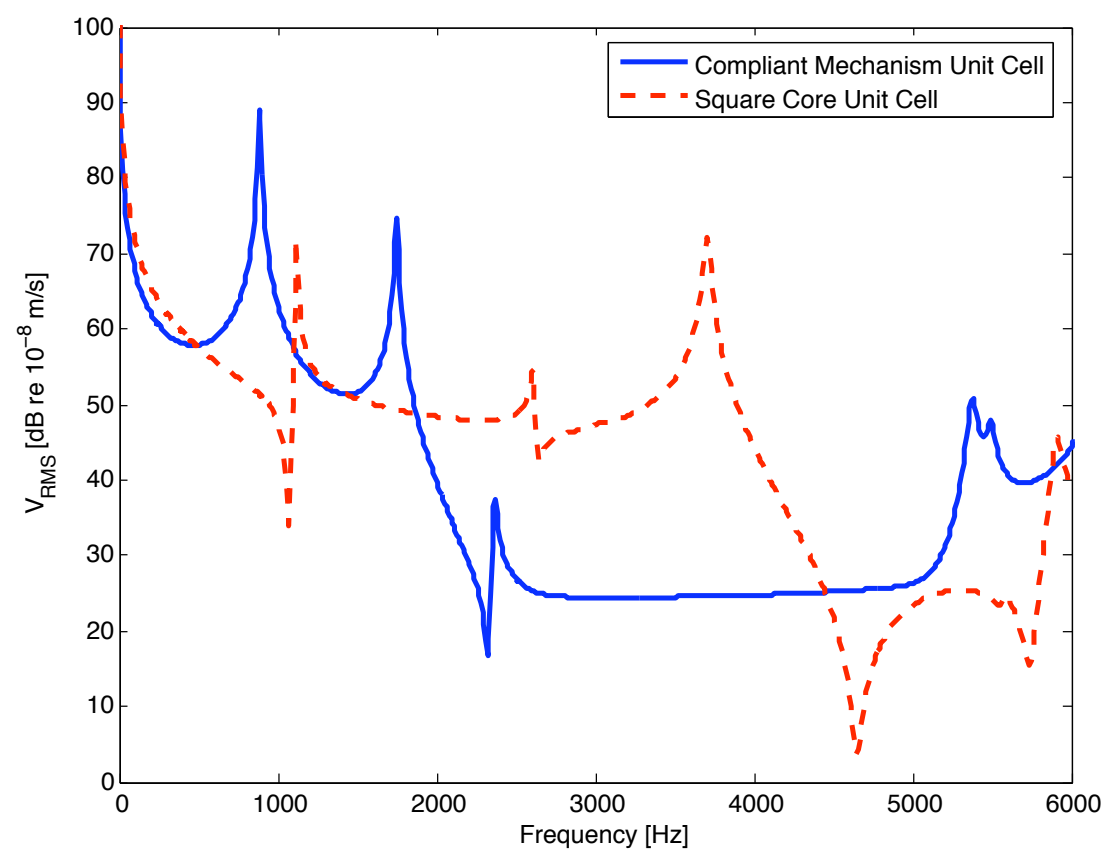

Figure 3.11: Top layer RMS velocity comparison for unit cell 


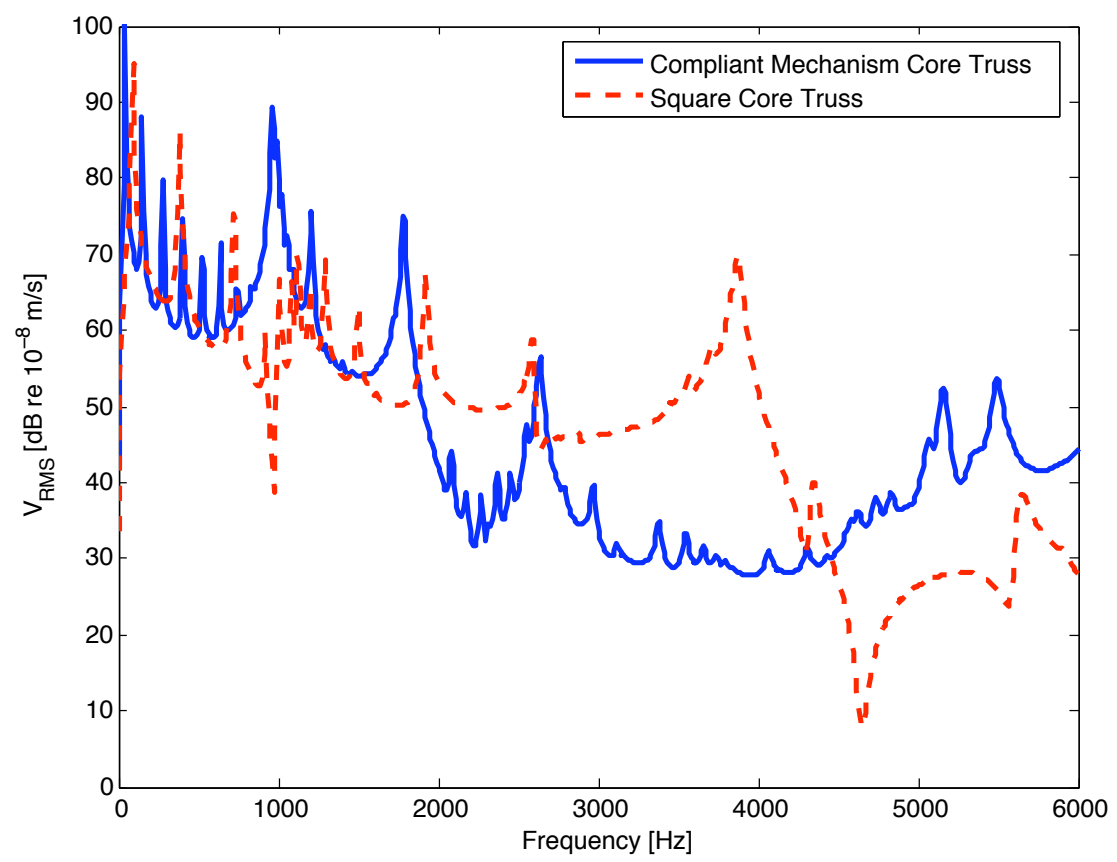

Figure 3.12: Top layer RMS velocity comparison for structure

Supplementing the FRF and RMS transverse velocity results, the deformed shapes of the unit cell can also be computed along with element energetics to gain additional knowledge of the behavior of the structure. The total energy, $E$, of each element is evaluated using the spectral stiffness matrix for a given frame element once the spectral nodal displacement vector for that element has been computed.

$$
E_{e}=\left|\frac{1}{2} \hat{\mathbf{d}}_{\mathrm{f}}^{\mathrm{T}} \hat{\mathbf{K}}_{\mathrm{f}} \hat{\mathbf{d}}_{\mathrm{f}}\right|
$$

The deformed shape and total energy at several frequencies provide insight into the compliant mechanism unit cell performance, Figures 3.13 through 3.16. Thicker lines denote greater energy content. Within the attenuation zone (Figures 3.13 and 3.14) energy isolation is evident, corresponding to the propagation of a transverse wave in the bottom layer and the mechanism acting as an "inversor." At the first mechanism resonance (Figure 3.15) in-phase behavior is observed. Outside the attenuation zone (Figure 3.16) energy flows into the core and top layer. 
In the calculation of the far-field SPL distribution, Eqn (3.17) is summed from $m=-100$ to $m=100$ and each structure is assumed to be in air with $\rho_{f}=1.2 \mathrm{~kg} / \mathrm{m}^{3}$ and $c_{a}=343 \mathrm{~m} / \mathrm{s}$. The far-field SPL is computed over a $4 \mathrm{~m}$ by $4 \mathrm{~m}$ grid having a $0.0125 \mathrm{~m}$ spatial resolution in the $x$ and $y$ directions. A comparison is made of the far-field SPL distribution in the fluid region above both structures at $1780 \mathrm{~Hz}$ and $3870 \mathrm{~Hz}$. Figures 3.17 and 3.18, respectively illustrate that the compliant mechanism structure indeed produces a higher SPL distribution at $1780 \mathrm{~Hz}$, as expected based on the RMS normal velocity response. Note that the structure top layer is located between $-1 \mathrm{~m}$ and $+1 \mathrm{~m}$ along the bottom horizontal axis of these figures, and that the SPL radiation patterns are dependent upon the unique deformation of each structure. Furthermore, a reduced SPL distribution of nearly $40 \mathrm{~dB}$ at $3870 \mathrm{~Hz}$, within the attenuation zone, is seen in Figures 3.19 and 3.20. These results represent a significant reduction in transmitted sound relative to conventional square core truss structures. 


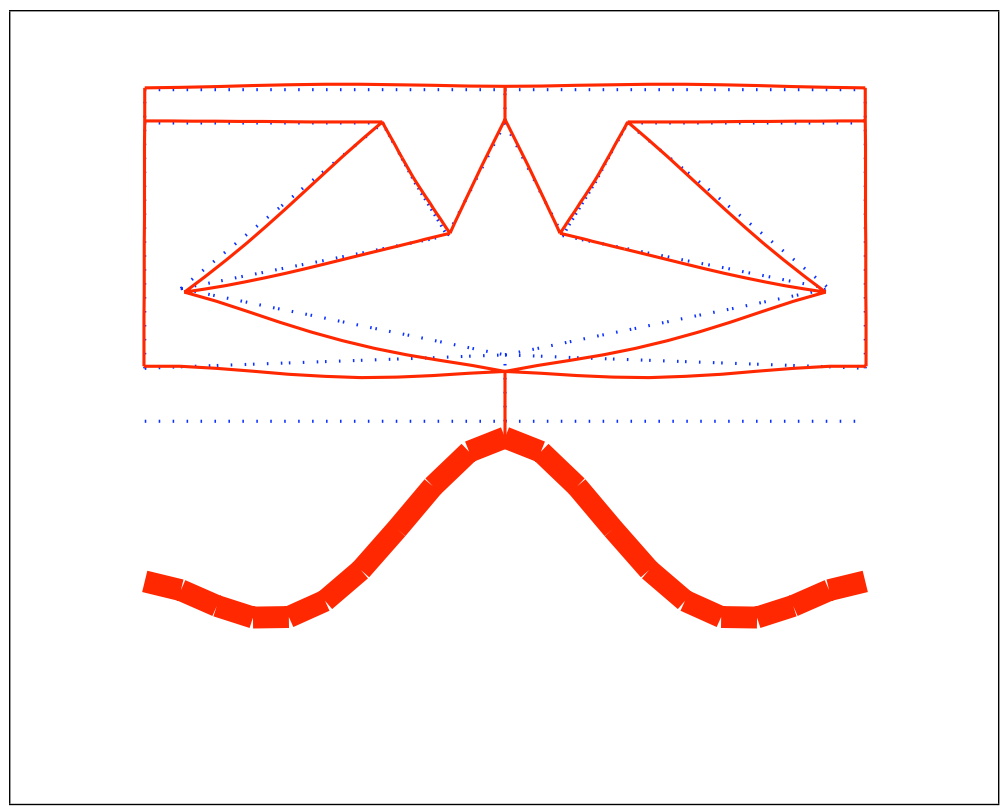

Figure 3.13: Compliant mechanism unit cell deformed shape and energy distribution at $3500 \mathrm{~Hz}$ (dotted lines denote undeformed configuration, continuous lines denote deformed configuration)

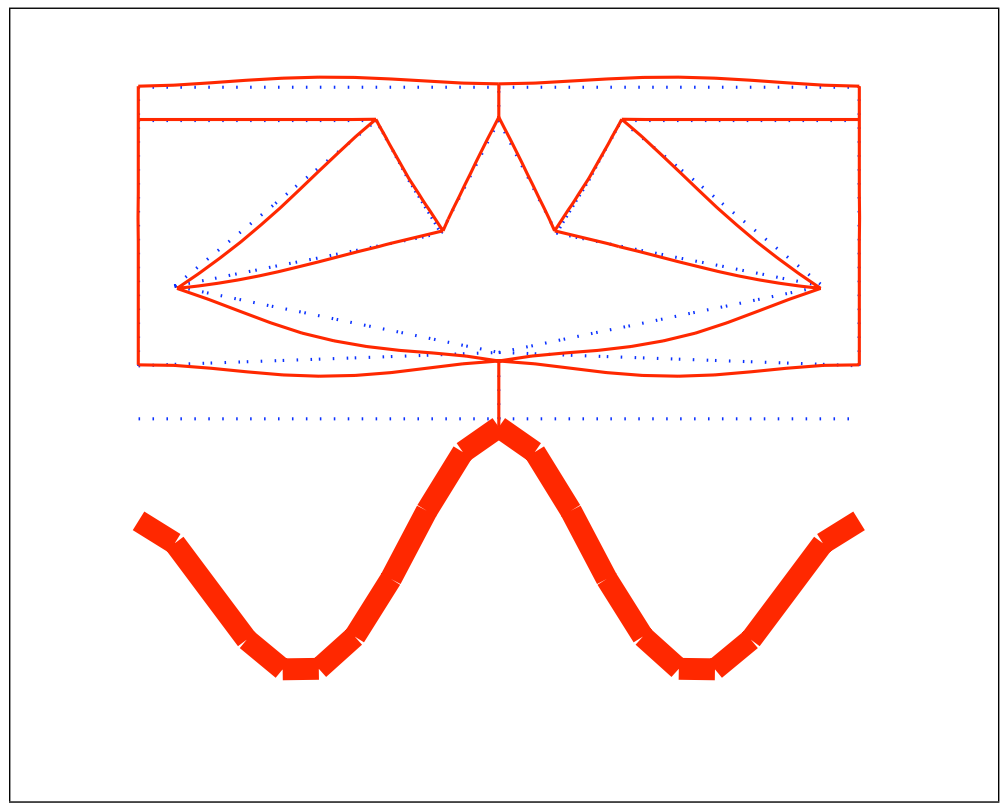

Figure 3.14: Compliant mechanism unit cell deformed shape and energy distribution at $4500 \mathrm{~Hz}$ (dotted lines denote undeformed configuration, continuous lines denote deformed configuration) 


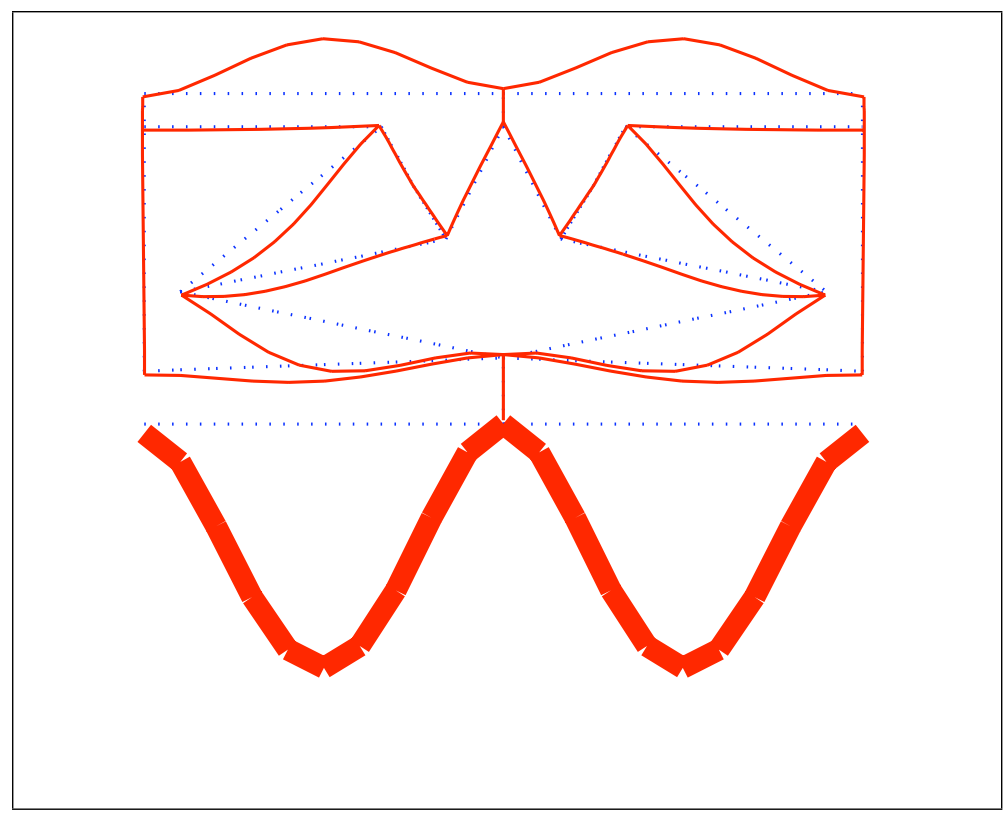

Figure 3.15: Compliant mechanism unit cell deformed shape and energy distribution at $5220 \mathrm{~Hz}$ (dotted lines denote undeformed configuration, continuous lines denote deformed configuration)

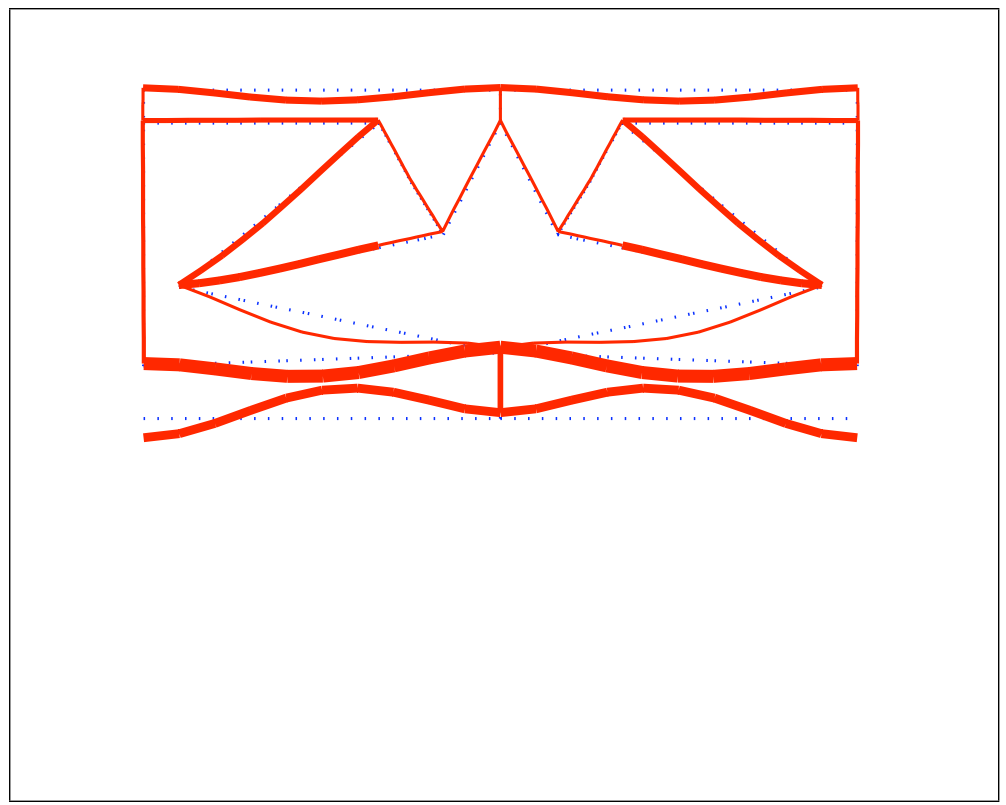

Figure 3.16: Compliant mechanism unit cell deformed shape and energy distribution at $\sim 6000 \mathrm{~Hz}$ (dotted lines denote undeformed configuration, continuous lines denote deformed configuration) 


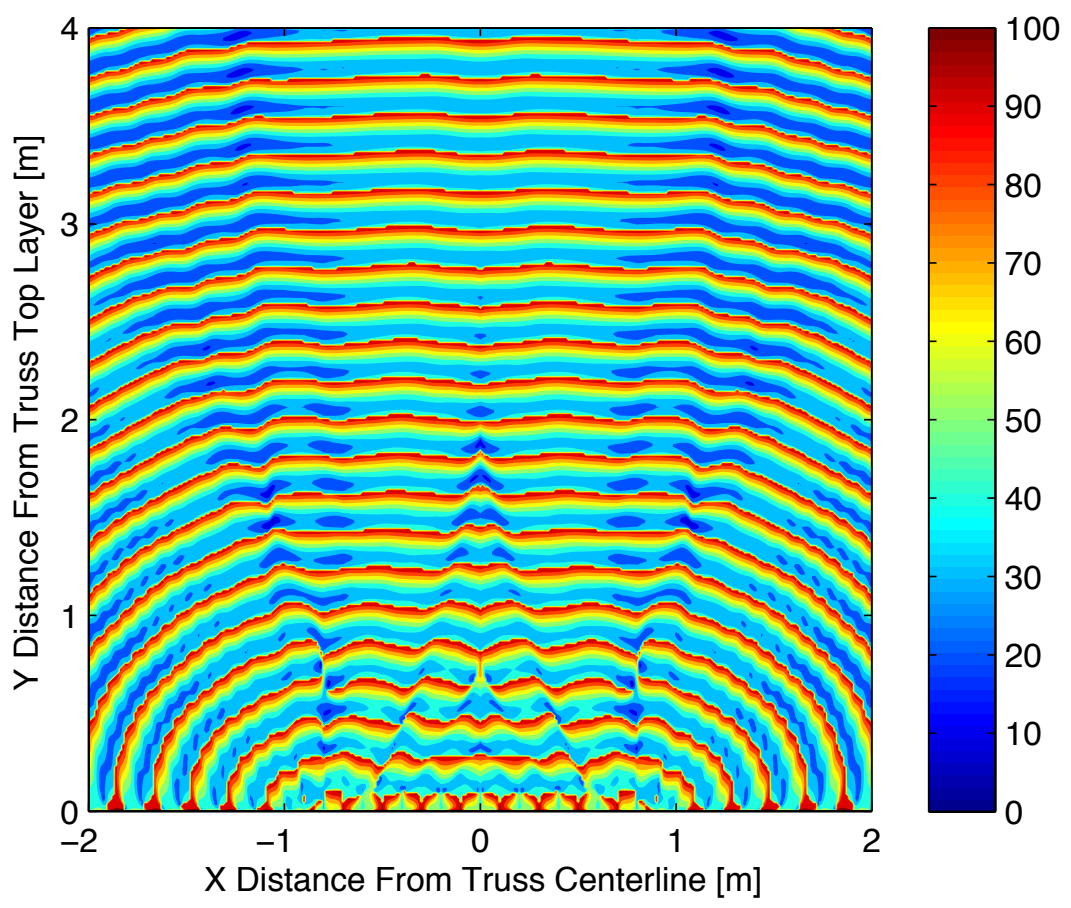

Figure 3.17: Square core structure SPL at $1780 \mathrm{~Hz}$ - units: dB re 20E-6 Pa

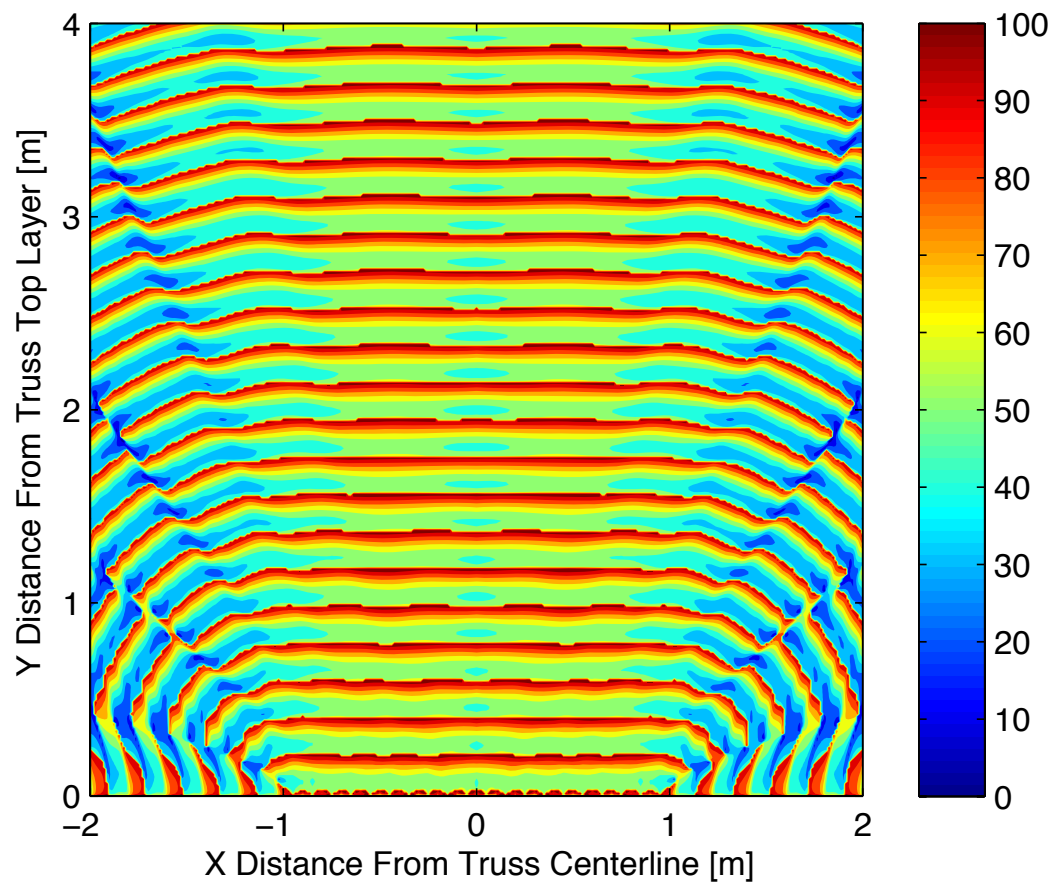

Figure 3.18: Compliant mechanism core structure SPL at $1780 \mathrm{~Hz}$ - units: dB re 20E-6 Pa 


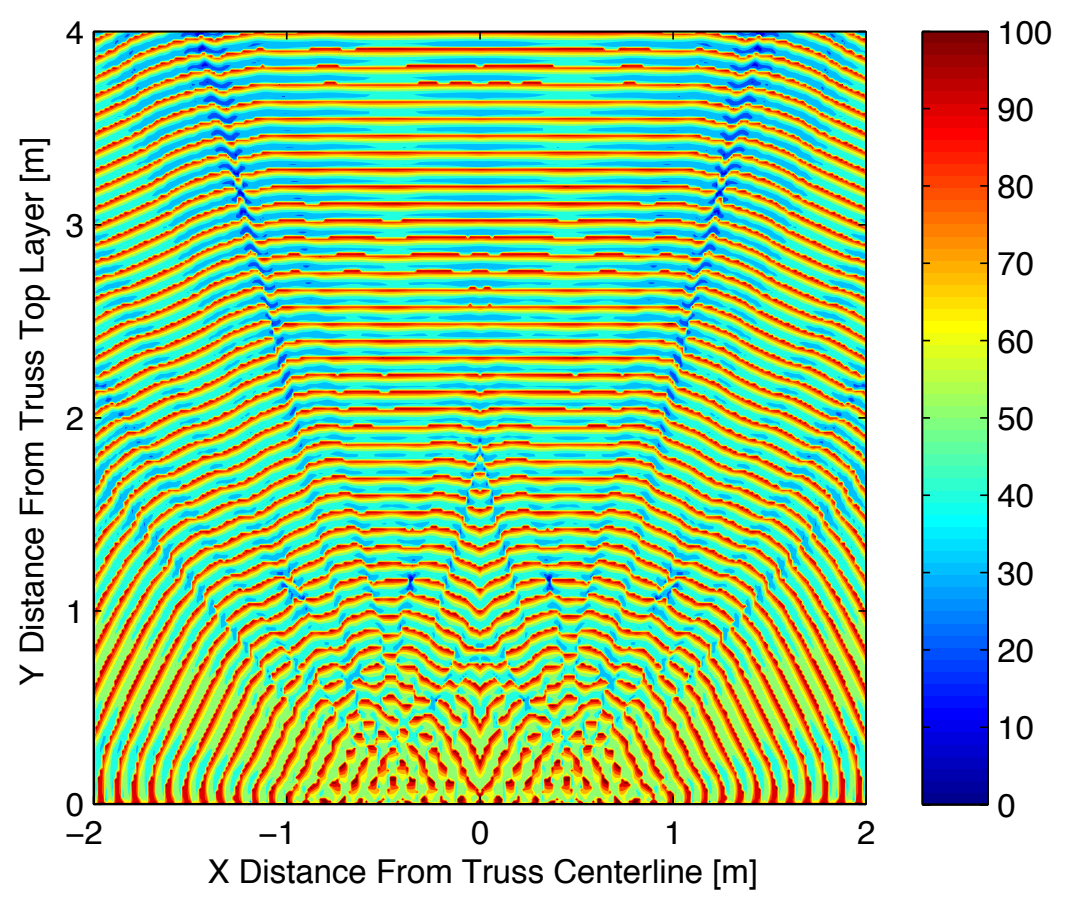

Figure 3.19: Square core structure SPL at $3870 \mathrm{~Hz}$ - units: dB re 20E-6 Pa

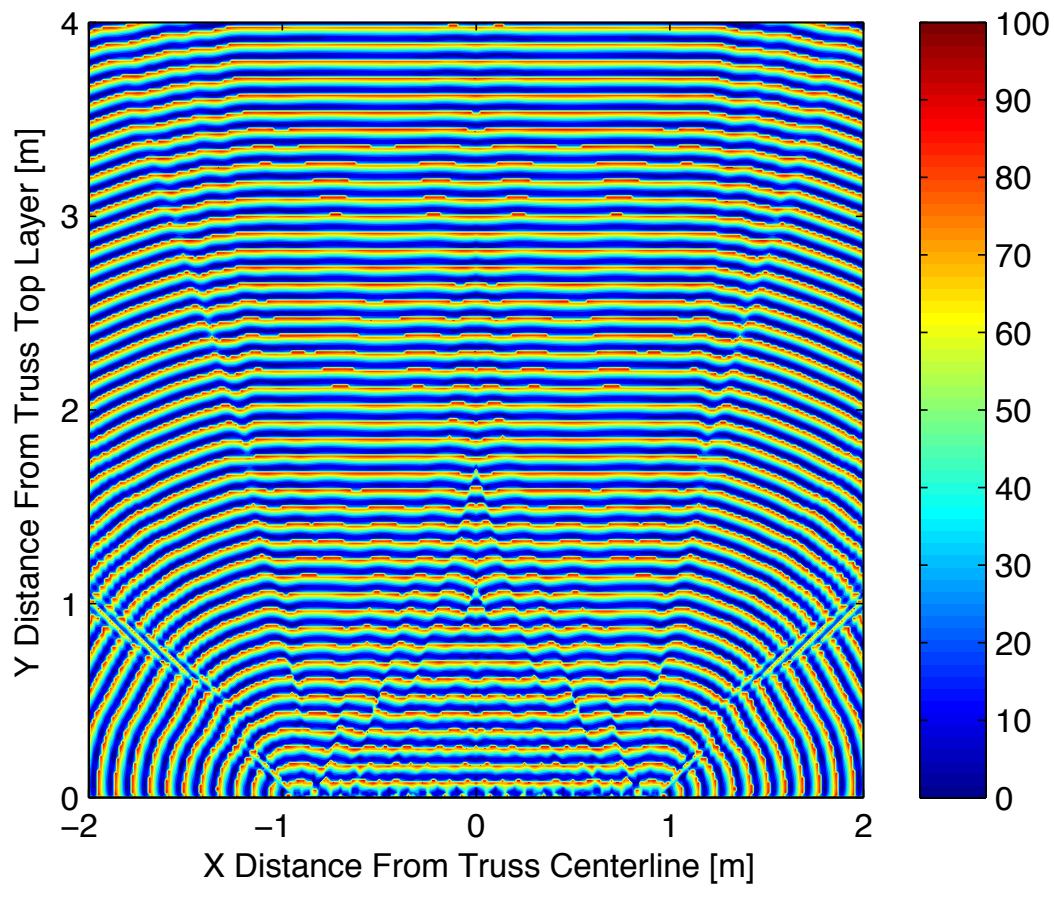

Figure 3.20: Compliant mechanism core structure SPL at $3870 \mathrm{~Hz}$ - units: dB re $20 \mathrm{E}-6 \mathrm{~Pa}$ 
To ensure accurate calculation of the far-field SPL distribution the convergence of Eqn (3.17) was examined. The far-field SPL distribution for the compliant mechanism structure at $1700 \mathrm{~Hz}$ is compared in Figures 3.21 (a), (b), and (c) using values for $m$ in Eqn (3.17) of 10, 100, and 200, respectively. The percent difference between the far-field SPL distribution calculated using $m=10$ and $m=100$ is shown in Figure 3.22 (a). Significant differences are noticeable in peripheral regions beyond the edges of the structure above the rigid baffles. The percent difference between the far-field SPL calculated using $m=100$ and $m=200$ is shown in Figure 3.22 (b). Only slight differences are noticeable, thus verifying the adequacy of using $m=100$ in the final computation of the far-field SPL distributions.

Spatial convergence was also examined by comparing the far-field SPL distribution obtained using a grid having a $0.0125 \mathrm{~m}$ spatial resolution versus a $0.00625 \mathrm{~m}$ spatial resolution. The far-field SPL of the compliant mechanism structure was computed over a $1 \mathrm{~m}$ square grid at $3870 \mathrm{~Hz}$ for each resolution, as shown in Figure 3.23. Minor differences in the far-field SPL distribution are noticeable, substantiating the use of a $0.0125 \mathrm{~m}$ resolution. Note, however, that as even higher frequencies are considered a finer step size must be utilized to sufficiently capture wave propagation. 


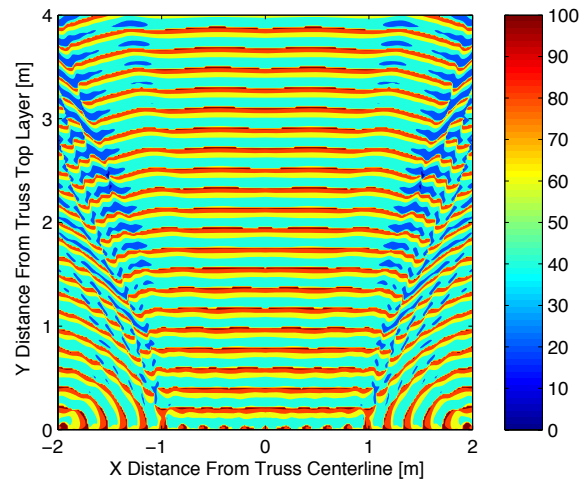

(a) $m=10$

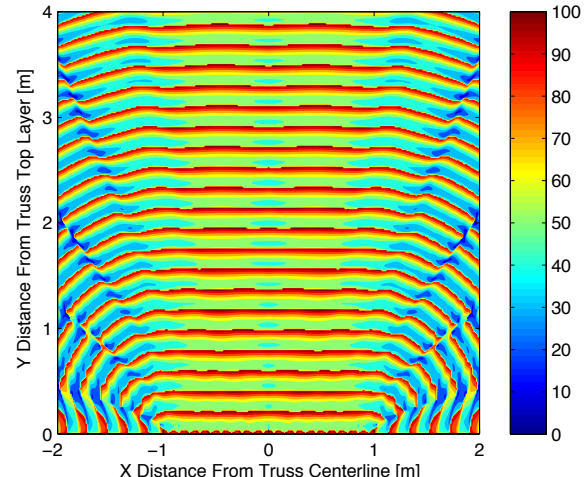

(b) $m=100$

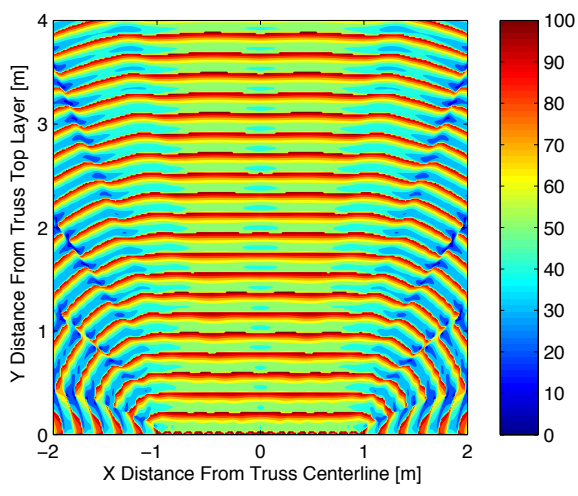

(c) $m=200$

Figure 3.21: Convergence of far-field SPL at $1700 \mathrm{~Hz}$ : (a) $m=10$, (b) $m=100$, and (c) $m=200$ - units: dB re $20 \mathrm{E}-6 \mathrm{~Pa}$

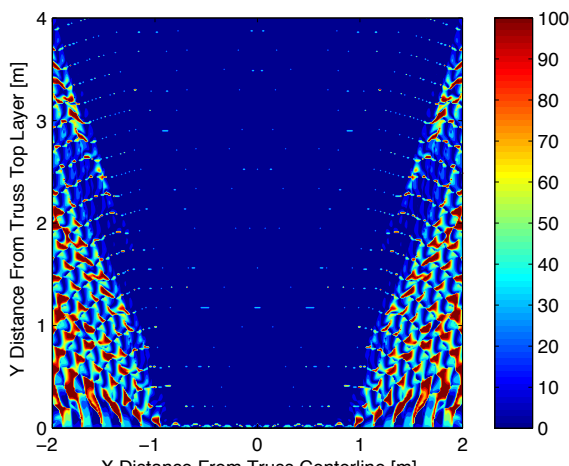

$\mathrm{X}$ Distance From Truss Centerline [m]

(a) $\%$ difference: $m=100$ and $m=10$

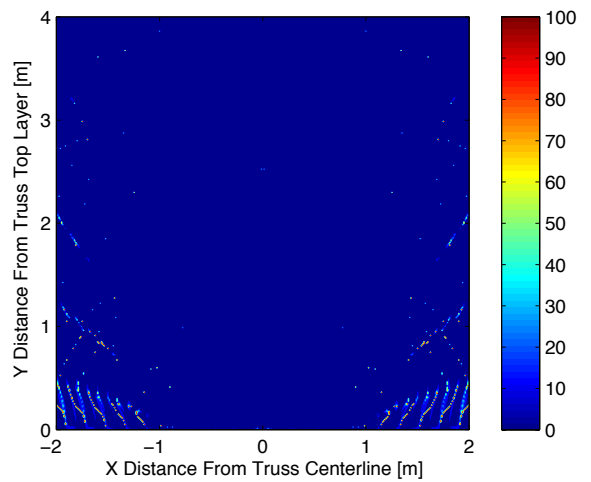

(b) $\%$ difference: $m=200$ and $m=100$

Figure 3.22: Percent difference between far-field SPL distribution at $1700 \mathrm{~Hz}$ : (a) $m$ $=100$ and $m=10$; (b) $m=200$ and $m=100$ 


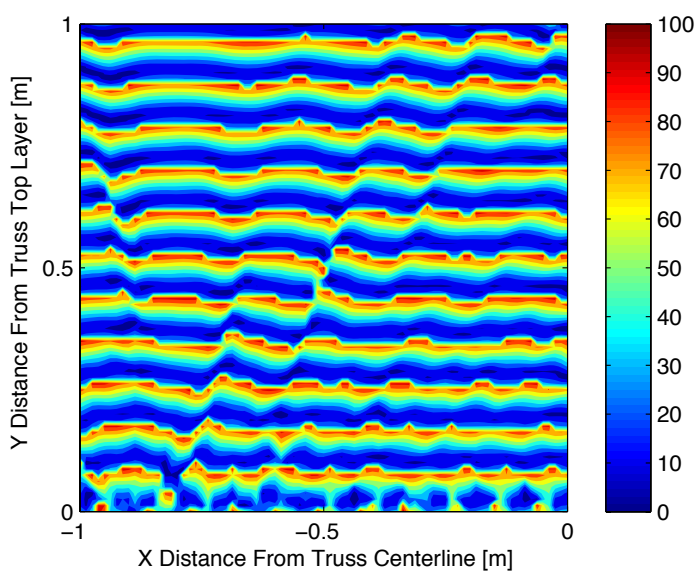

(a) $0.0125 \mathrm{~m}$ grid

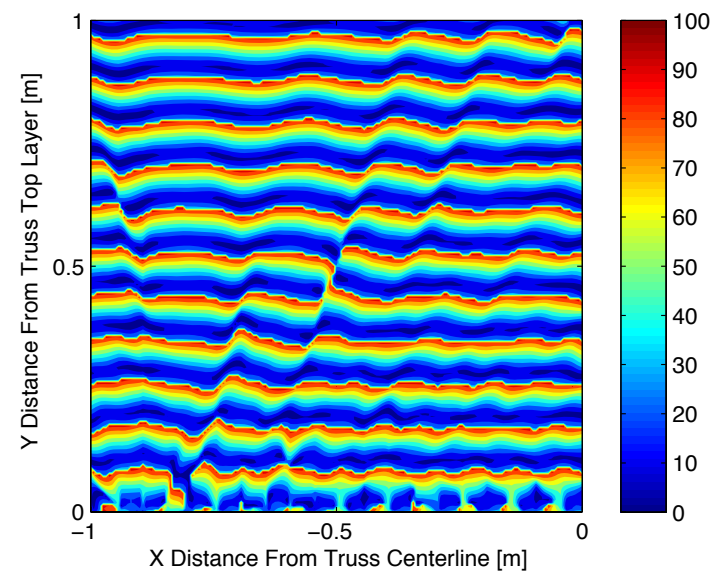

(b) $0.00625 \mathrm{~m}$ grid

Figure 3.23: Spatial convergence of far-field SPL distribution at $3870 \mathrm{~Hz}$ : (a) 0.0125 $\mathrm{m}$ resolution; (b) $0.00625 \mathrm{~m}$ resolution - units: dB re $20 \mathrm{E}-6 \mathrm{~Pa}$

(a) $40 \mathrm{~Hz}$

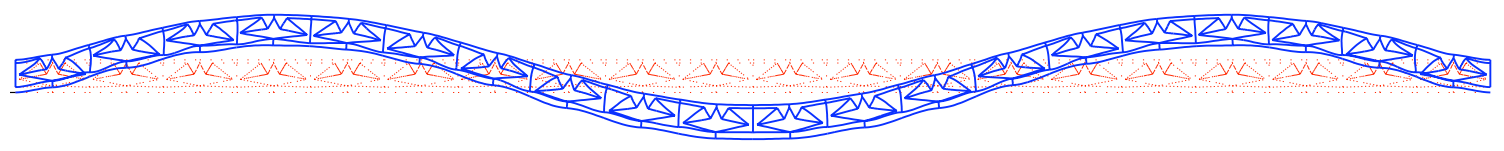

(b) $150 \mathrm{~Hz}$

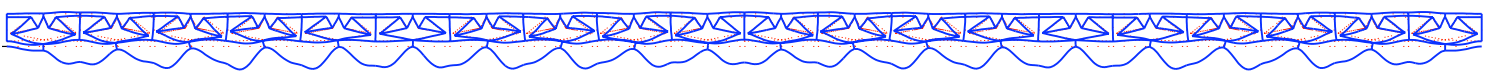

(c) $3000 \mathrm{~Hz}$

Figure 3.24: Deformed shapes of compliant mechanism structure: (a) $40 \mathrm{~Hz}$, (b) 150 $\mathrm{Hz}$, and (c) $3000 \mathrm{~Hz}$ (dotted lines denote undeformed configuration, continuous lines denote deformed configuration) 
Lastly, moving to a global view, deformed configurations of the compliant mechanism structure are given in Figures 3.24 (a), (b), and (c) at frequencies of $40 \mathrm{~Hz}$, $150 \mathrm{~Hz}$, and $3000 \mathrm{~Hz}$, respectively. It is again evident that as frequency increases the deformed shape transitions from global to local. Specific to the design of this structure, the local deformation at $3000 \mathrm{~Hz}$ illustrates the propagation of a transverse wave traveling through the bottom layer.

\subsubsection{Concept \& Definitions: Compliant Mechanism 2}

In this section another "inversor" compliant mechanism topology is integrated into a unit cell as independent verification of the analysis and design methodology introduced thus far. Namely, a mechanism that is commonly described as a rhombus straight-line linkage is investigated. Straight-line mechanisms served their role in

American history through power machines (e.g. 18th century Watt-Boulton steam engines). Functionally, they can be used to convert the linear motion from an oscillating piston into the rocking motion of a balance arm. The rhombus straight-line linkage is inspired by Model S39 from the Reuleaux Collection of Kinematic Mechanisms at Cornell University [83], and it has been shown to have an optimum topology for compliant mechanisms with a large mechanical advantage by Larsen et al. [57]. While this mechanism topology differs from the one presented in Section 3.2.1, it embodies the same amplification principles. A diagram of one half of the rigid link rhombus straight-line mechanism is given in Figure 3.25. The compliant mechanism unit cell embodiment is shown in Figure 3.26. The equation for calculating the mechanical advantage, $\mathrm{MA}_{\mathrm{CM} 2}$, of this rhombus rigid link mechanism is given as

$$
\mathrm{MA}_{\mathrm{CM} 2}=\frac{\mathrm{v}_{\text {in }}}{\mathrm{v}_{\text {out }}}=\frac{r_{\text {in }}}{r_{\text {out }}} \frac{((1,5)-(3,5))}{((1,3)-(3,5))}
$$




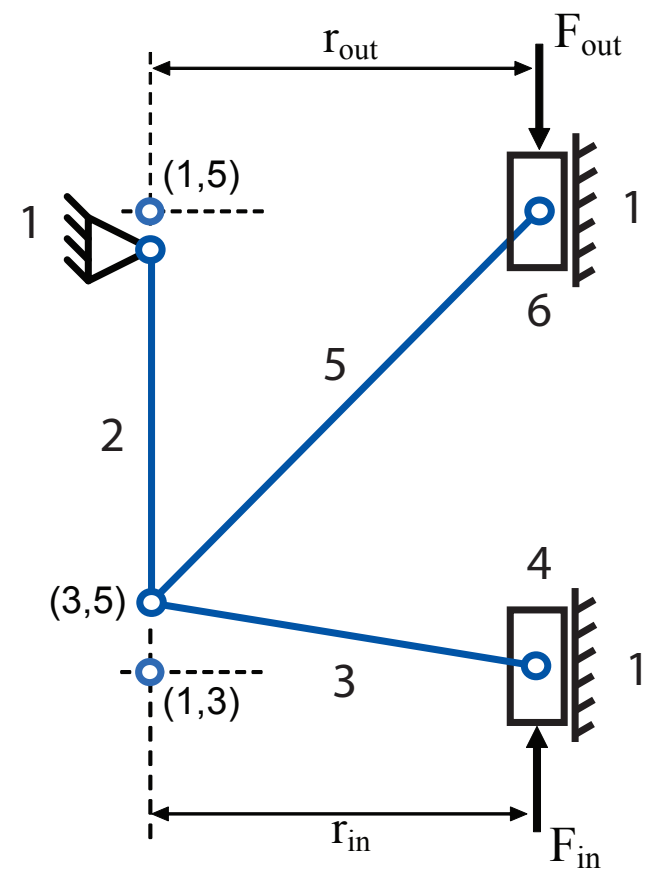

Figure 3.25: Rigid link diagram of one half of rhombus mechanism (numbers denote links, $(\cdot, \cdot)$ denote instant centers)

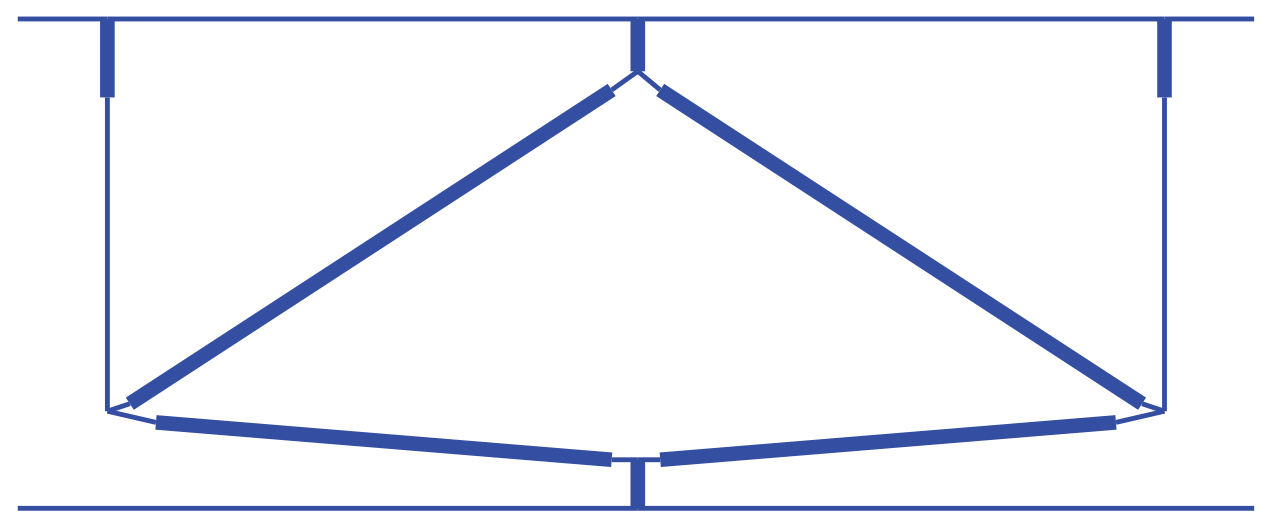

Figure 3.26: Rhombus compliant mechanism unit cell model

In the compliant mechanism unit cell model shown in Figure 3.27, the sliders in the rigid link diagram are enforced through symmetry about the vertical centerline of the unit cell. Moreover, the ground elements for the mechanism, elements 3 and 23, are attached to the unit cell top layer. As explained in Section 3.2.1, when the 


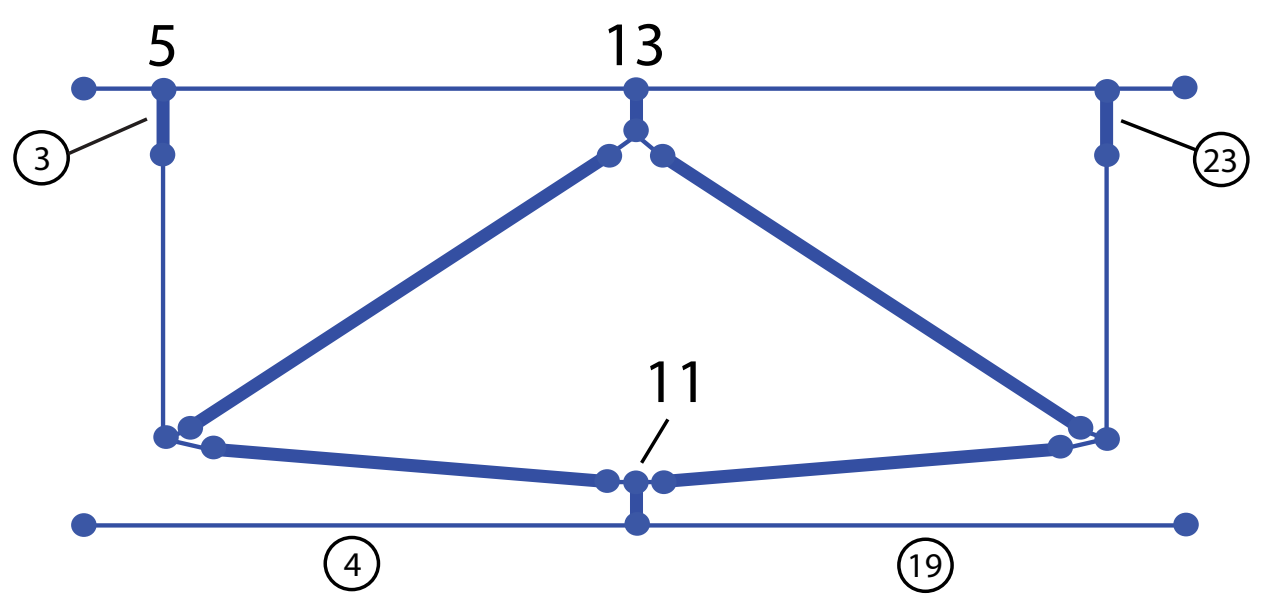

Figure 3.27: Rhombus compliant mechanism unit cell model (numbers that are circled denote elements, numbers that are not circled denote nodes)

unit cell bottom layer elements (i.e. 4 and 19) are subjected to an incident pressure wave, this connectivity scheme allows transverse waves to propagate through the bottom layer unimpeded, evoking mechanical advantage through passive mechanism actuation.

The frequency response function (FRF) output to input amplitude and phase of this compliant mechanism unit cell model are defined by the following relationships

$$
\text { Amplitude }=\left|\frac{\hat{v}_{13}-\hat{v}_{5}}{\hat{v}_{11}-\hat{v}_{5}}\right| \quad ; \quad \text { Phase }=\frac{180}{\pi}\left[\angle\left(\hat{v}_{13}-\hat{v}_{5}\right)-\angle\left(\hat{v}_{11}-\hat{v}_{5}\right)\right]
$$

where the unit cell output is taken as the transverse spectral displacement of node 13 relative to node 5, while the input is taken as the transverse spectral displacement of node 11 relative to node 5 . Node 5 is selected since it represents part of the ground of the mechanism. These relationships provide insight into the performance of the compliant mechanism unit cell distinguishing out-of-phase, non-resonant performance versus in-phase, resonant performance. 


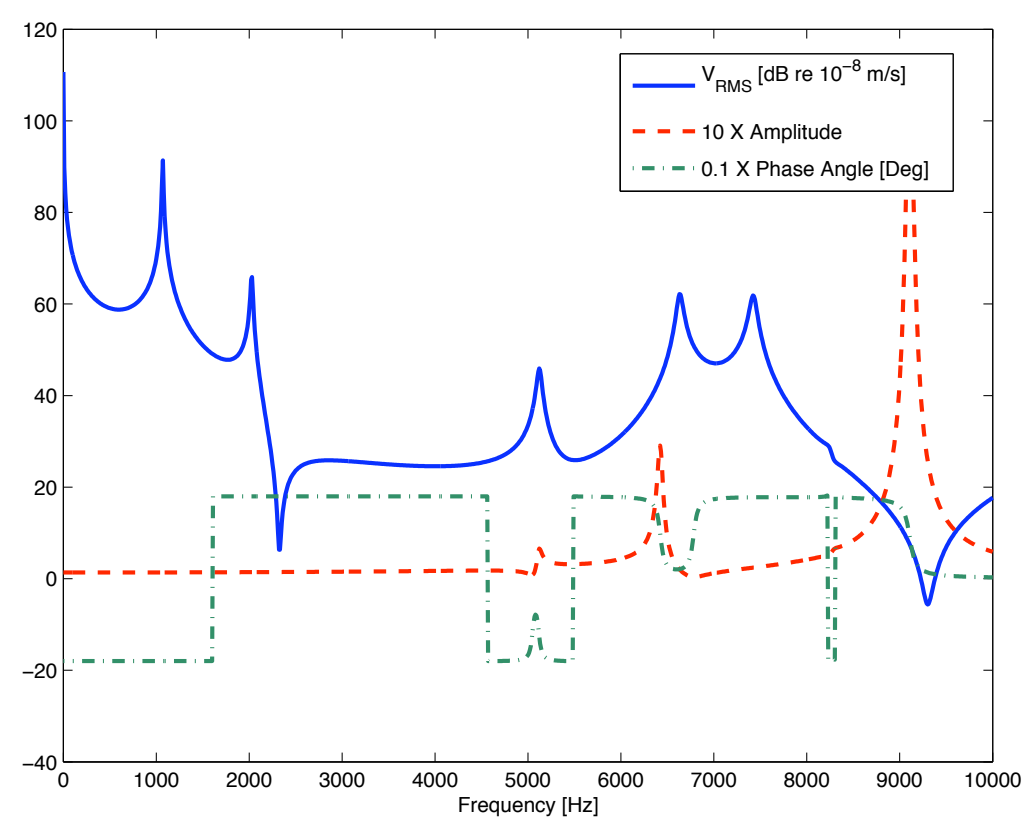

Figure 3.28: Rhombus compliant mechanism unit cell FRF

\subsubsection{Computational Results: Compliant Mechanism 2}

Unit cell dimensions of $0.1 \mathrm{~m}$ by $0.05 \mathrm{~m}$ are used, which leads to a compliant mechanism having a mechanical advantage of 7, per Eqn (3.19). In Figure 3.27 thicker lines represent stiffer elements having a width of $5 \mathrm{~mm}$. Thinner lines represent elements having greater compliance and a width of $2.5 \mathrm{~mm}$. The width of members within this compliant mechanism unit cell is consistent with the width of members within the prior two unit cells that have already been analyzed. The sparse topology of this compliant mechanism unit cell leads to a total mass that is only 1.13 times that of the square core unit cell.

All of the analytical tools developed for the square core structure and the first compliant mechanism structure are used to compute the structural dynamic response of the rhombus compliant mechanism unit cell from $0 \mathrm{~Hz}$ to $10 \mathrm{kHz}$, evaluated in $10 \mathrm{~Hz}$ frequency steps. The FRF response is shown in Figure 3.28. The structural 
dynamic response of the rhombus compliant mechanism unit cell design mimics that of the first compliant mechanism design given in Sections 3.2.1-3.2.3, serving as independent verification of the analysis and design methodology.

Below $2320 \mathrm{~Hz}$ two peaks in response are visible at $1070 \mathrm{~Hz}$ and $2030 \mathrm{~Hz}$. Above $2320 \mathrm{~Hz}$ two major attenuation zones are present spanning the frequency ranges of 2320-5000 Hz and 9240-9600 Hz. At the starting frequencies of these attenuation zones, the wavelength of vibration for transverse waves in the unit cell bottom layer is equal to the unit cell width and half-width (i.e. $\lambda=0.1 \mathrm{~m}$ and $\lambda=0.05 \mathrm{~m}$ ), respectively, per Eqn (3.16). Within the first attenuation zone the compliant mechanism demonstrates out-of-phase, non-resonant (i.e. small amplitude) behavior, as expected. Between the two attenuation zones (i.e. 5000-9240 Hz) in-phase, resonant behavior is exemplified by peaks in the RMS transverse velocity response, increases in the FRF amplitude, and a FRF phase that transitions away from 180 degrees. Similarly, a significant peak in FRF amplitude is located in close proximity to the start of the second attenuation zone. This phenomenon is better understood through inspection of the deformed shapes of the unit cell. Nonetheless, significant reduction in RMS normal velocity response is still present within the second attenuation zone.

Shown in Figures 3.29 through 3.35, at several frequencies, are the rhombus compliant mechanism unit cell deformed shape and total energy distribution. Thicker lines again denote greater energy content. Within the first attenuation zone (Figures 3.29 and 3.30) energy isolation and the propagation of a transverse wave in the bottom layer are evident. The input and output of the compliant mechanism unit cell are out-of-phase corresponding to the mechanism acting as an "inversor." At the first mechanism resonance of $5110 \mathrm{~Hz}$ (Figure 3.31) larger amplitude deformation of the output relative to the input is observed corresponding to the peak in RMS 
velocity response in Figure 3.28. At $6620 \mathrm{~Hz}$ (Figure 3.32) in-phase behavior is visible and energy flows throughout the core and top layer. The peak in RMS normal velocity response at $7420 \mathrm{~Hz}$ in Figure 3.28 is a consequence of energy flowing into the unit cell core (Figure 3.33). The second attenuation zone begins at $9240 \mathrm{~Hz}$ (Figure 3.34). Despite the fact that the input and output are shown to move in-phase in Figure 3.28, the deformed shape clarifies the fact that the mechanism is still acting as an "inversor." Close examination of this figure reveals the presence of localized deformations of the mechanism links within the core, which counteract the in-phase movement of the input and output of the unit cell. Additionally, isolation occurs to confine energy content to the bottom layer of the structure. Finally, at $9600 \mathrm{~Hz}$ (Figure 3.35) in-phase behavior dominates these localized core deformations despite continued energy isolation. This behavior leads to the eventual rise in RMS normal velocity response shown in Figure 3.28.

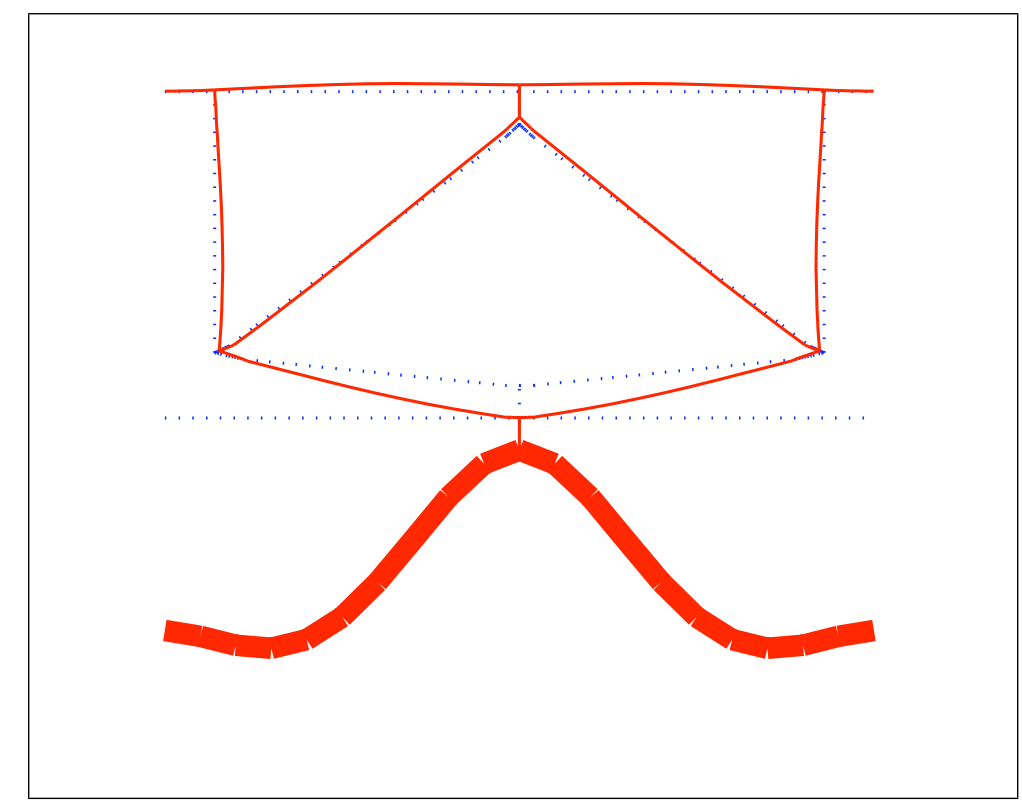

Figure 3.29: Rhombus compliant mechanism unit cell deformed shape and energy distribution at $3000 \mathrm{~Hz}$ (dotted lines denote undeformed configuration, continuous lines denote deformed configuration) 


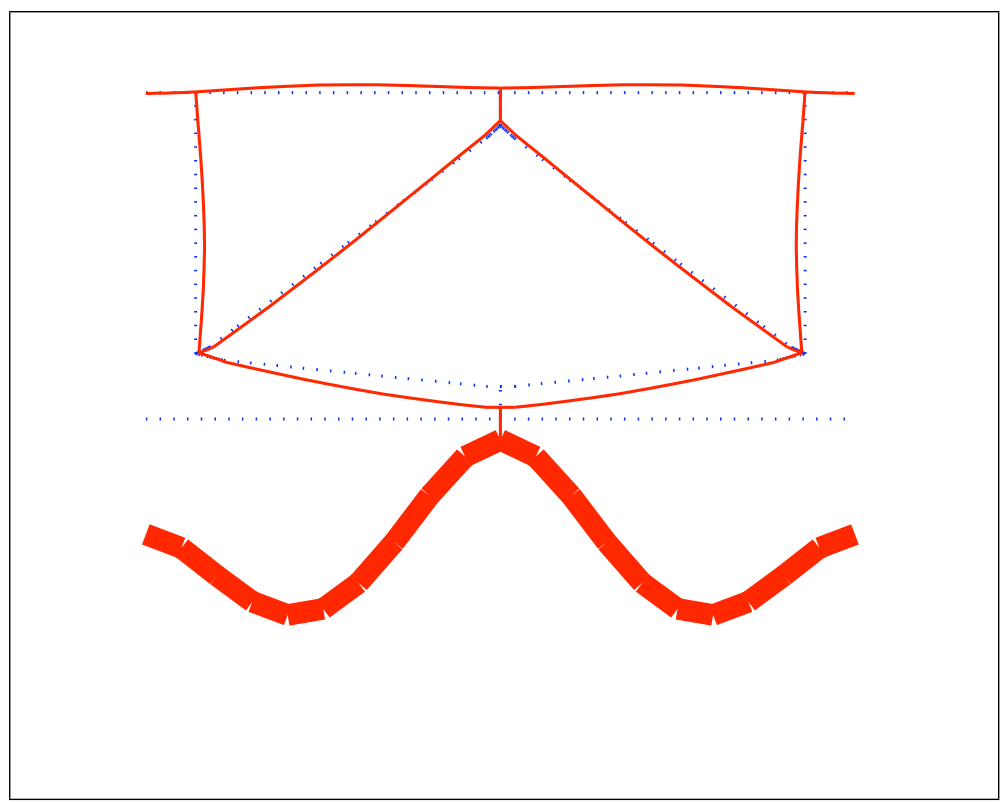

Figure 3.30: Rhombus compliant mechanism unit cell deformed shape and energy distribution at $4000 \mathrm{~Hz}$ (dotted lines denote undeformed configuration, continuous lines denote deformed configuration)

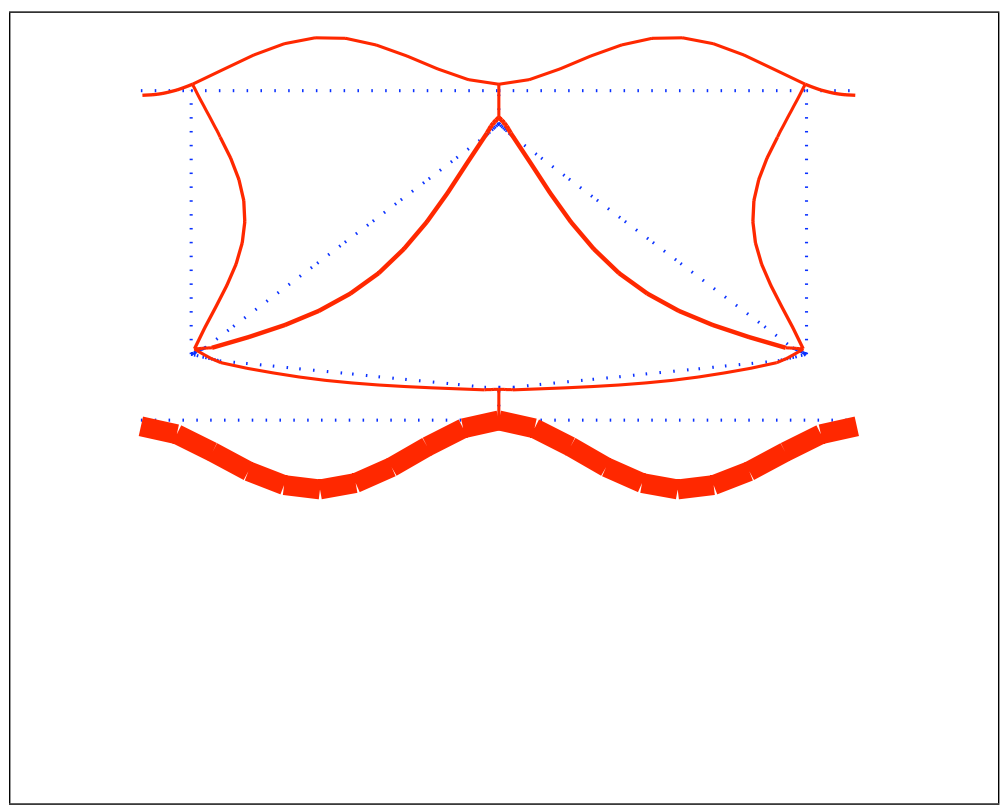

Figure 3.31: Rhombus compliant mechanism unit cell deformed shape and energy distribution at $5110 \mathrm{~Hz}$ (dotted lines denote undeformed configuration, continuous lines denote deformed configuration) 


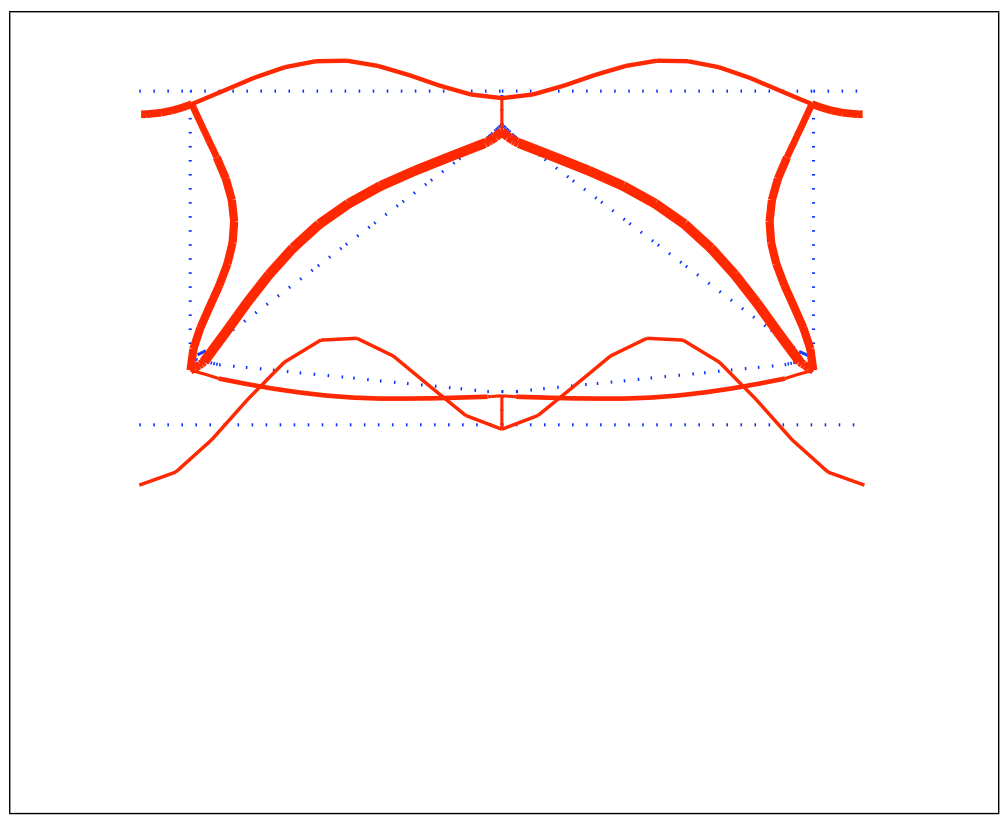

Figure 3.32: Rhombus compliant mechanism unit cell deformed shape and energy distribution at $6620 \mathrm{~Hz}$ (dotted lines denote undeformed configuration, continuous lines denote deformed configuration)

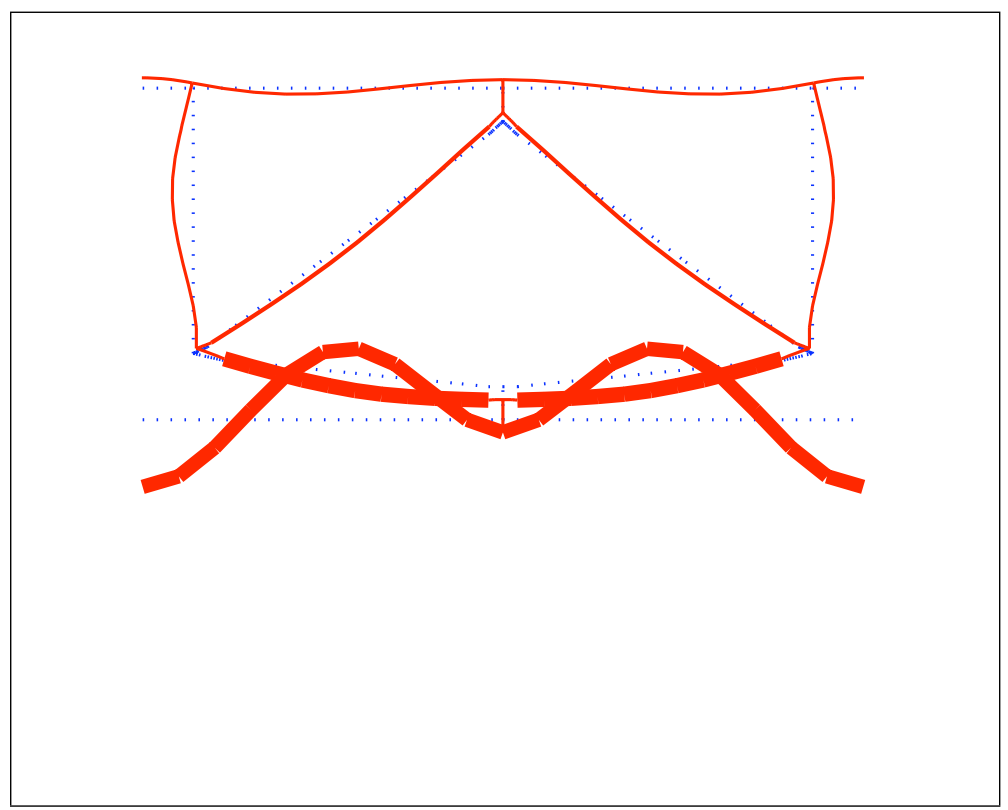

Figure 3.33: Rhombus compliant mechanism unit cell deformed shape and energy distribution at $7420 \mathrm{~Hz}$ (dotted lines denote undeformed configuration, continuous lines denote deformed configuration) 


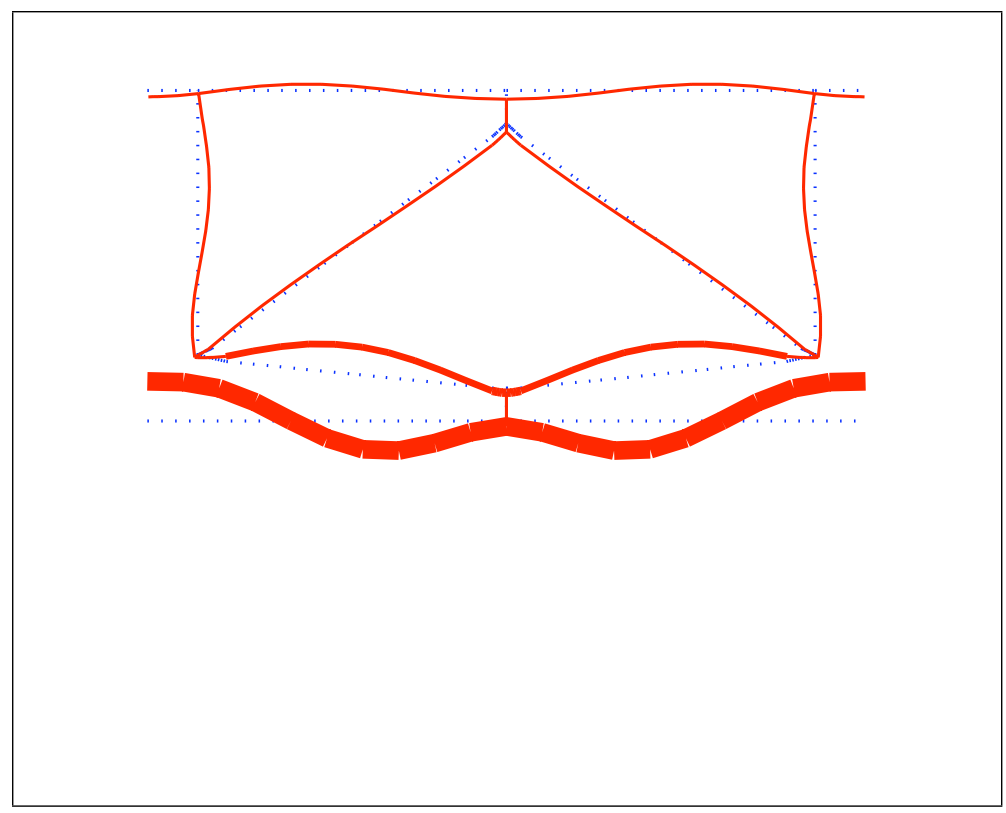

Figure 3.34: Rhombus compliant mechanism unit cell deformed shape and energy distribution at $9240 \mathrm{~Hz}$ (dotted lines denote undeformed configuration, continuous lines denote deformed configuration)

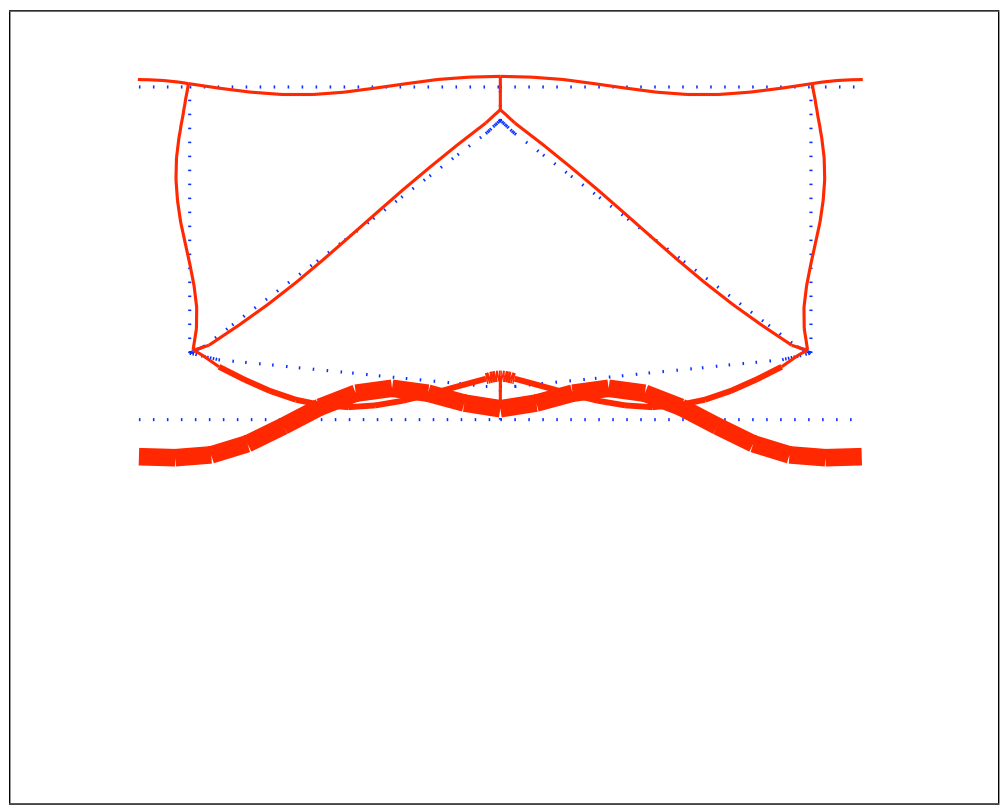

Figure 3.35: Rhombus compliant mechanism unit cell deformed shape and energy distribution at $9600 \mathrm{~Hz}$ (dotted lines denote undeformed configuration, continuous lines denote deformed configuration) 


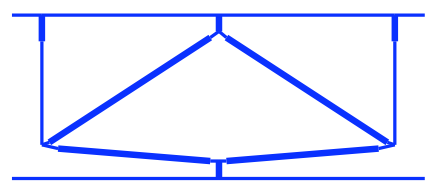

(a) $\mathrm{MA}=7$

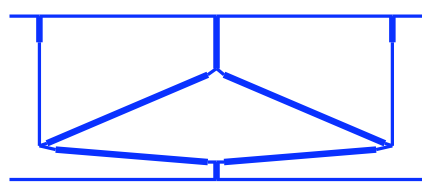

(b) $\mathrm{MA}=5$

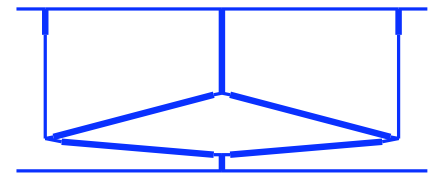

(c) $\mathrm{MA}=3$

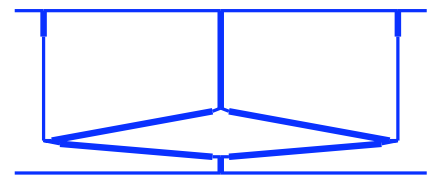

(d) $\mathrm{MA}=2$

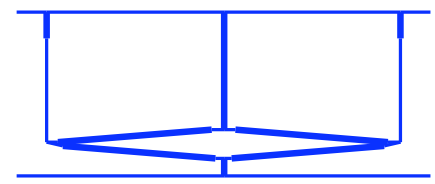

(e) $\mathrm{MA}=1$

Figure 3.36: Rhombus compliant mechanism unit cells with varying mechanical advantage: (a) $\mathrm{MA}=7$, (b) $\mathrm{MA}=5$, (c) $\mathrm{MA}=3$, (d) $\mathrm{MA}=2$, and (e) $\mathrm{MA}=1$

The sparseness of the topology of this integral compliant mechanism affords the benefit of easy shape modification to gain a greater understanding of the role of mechanical advantage as it pertains to the structural dynamic response of the unit cell. Adjusting the aspect ratio of the rhombus by moving the location of the top slider (i.e. link 6 in Figure 3.25) modifies the location of instant center $(1,5)$. In this manner, the mechanical advantage calculated in Eqn (3.19) can be decreased. Thus, consider five rhombus compliant mechanism unit cells having a respective mechanical advantage of $7,5,3,2$, and 1 in Figures 3.36 (a) through (e).

In Figure 3.37 the top layer RMS transverse velocity for the unit cells having MA values of $7,5,3$, and 2 is shown computed over a frequency range of $0 \mathrm{~Hz}$ to 15 $\mathrm{kHz}$, evaluated in $10 \mathrm{~Hz}$ increments. The extended upper limit on the middle frequency range is used in order to fully visualize the effect of decreasing the mechanical advantage on both attenuation zones.

Generally, a reduced unit cell mechanical advantage leads to an increase in the amplitude of response within both attenuation zones. Specifically, within the first 


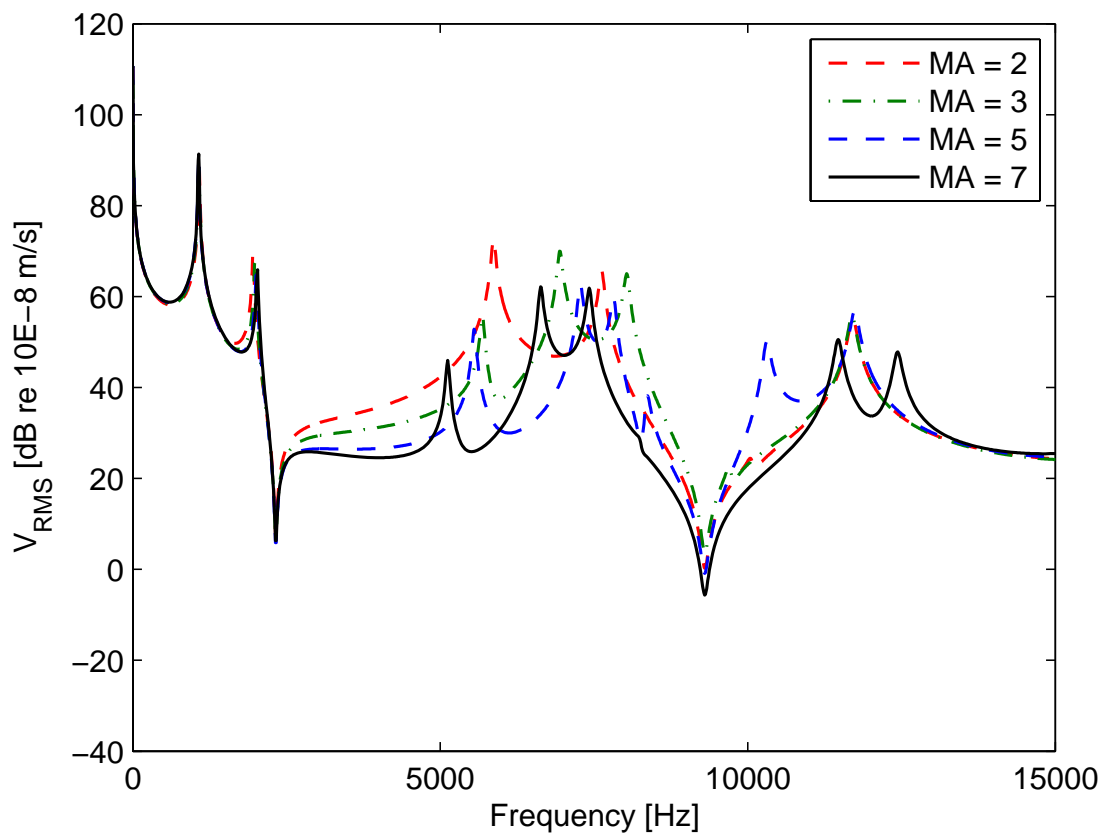

Figure 3.37: Top layer RMS velocity comparison for rhombus compliant mechanism unit cells with varying mechanical advantage

attenuation zone the slope of response increases as mechanical advantage is reduced; the resonant amplitude at the first attenuation zone cutoff frequency increases while shifting towards slightly higher frequencies. This behavior is an artifact of the increased transverse displacement of the output of the compliant mechanism unit cell relative to that of the input, which is illustrated by computing the FRF from $0 \mathrm{~Hz}$ to $8 \mathrm{kHz}$ for each of these unit cells. Comparing FRF amplitude response at $3600 \mathrm{~Hz}$ in Figures 3.38 through 3.41 reveals that amplitude increases as mechanical advantage decreases, as expected.

As mechanical advantage is reduced to a value of unity, dynamic behavior of the unit cell further changes as shown in Figure 3.42. However, observe that the resonant peak at the attenuation zone cutoff frequency (i.e. $4160 \mathrm{~Hz}$ ) has shifted much lower and does not follow the established trend. Examining the deformed shape and energy distribution at the cutoff frequency for all five unit cells reveals 


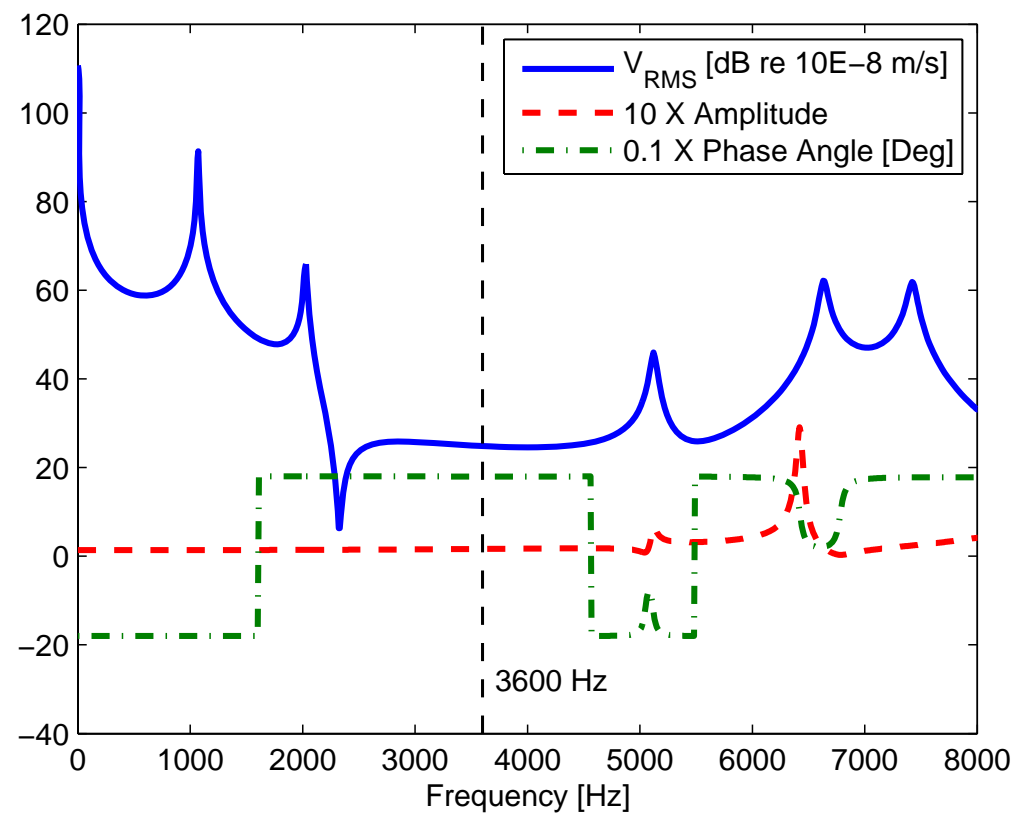

Figure 3.38: Rhombus compliant mechanism $(\mathrm{MA}=7)$ unit cell FRF

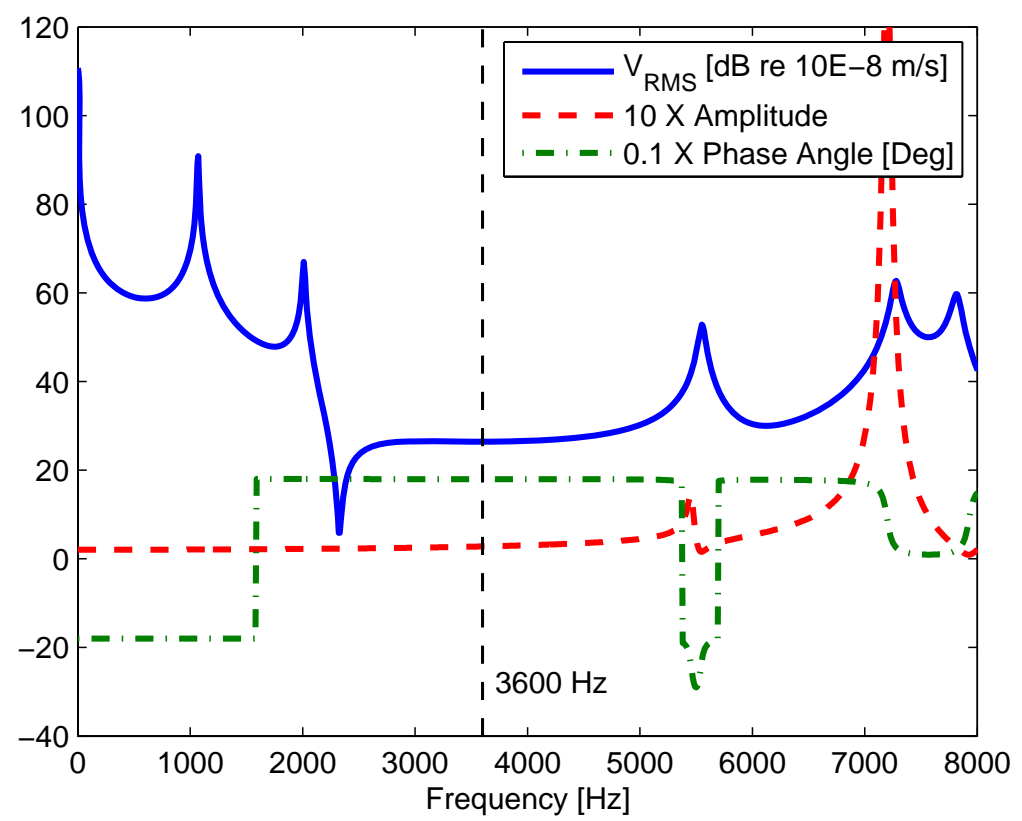

Figure 3.39: Rhombus compliant mechanism $(\mathrm{MA}=5)$ unit cell FRF 


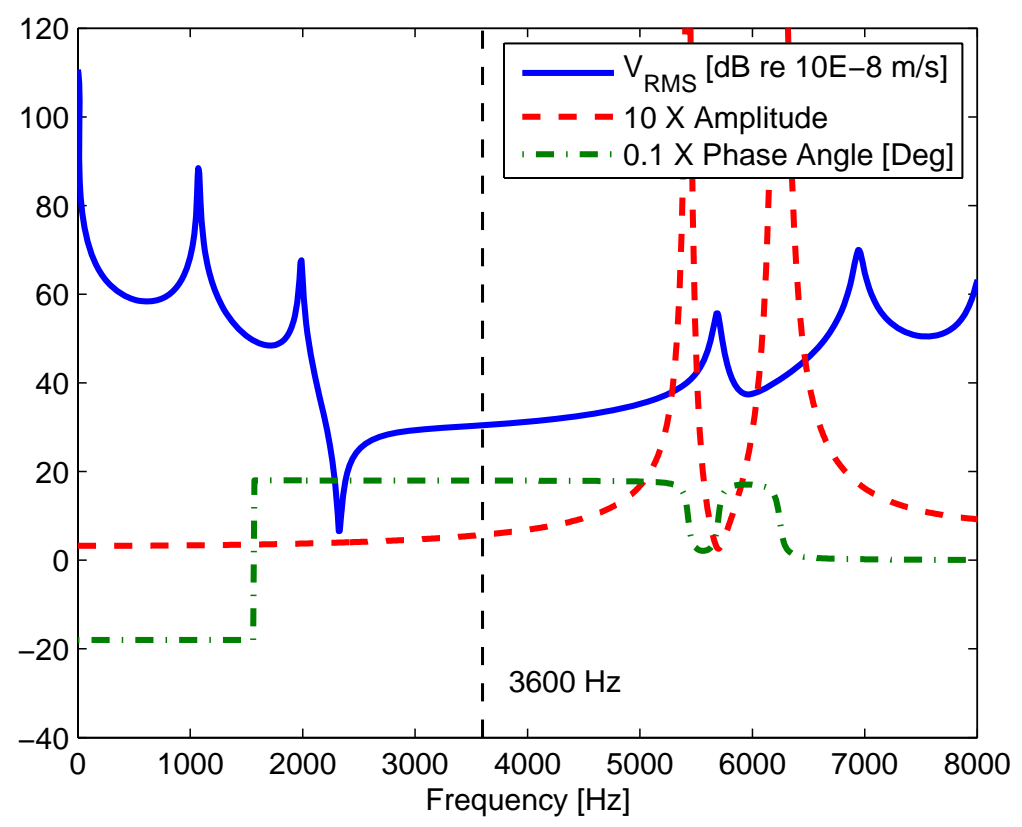

Figure 3.40: Rhombus compliant mechanism $(\mathrm{MA}=3)$ unit cell FRF

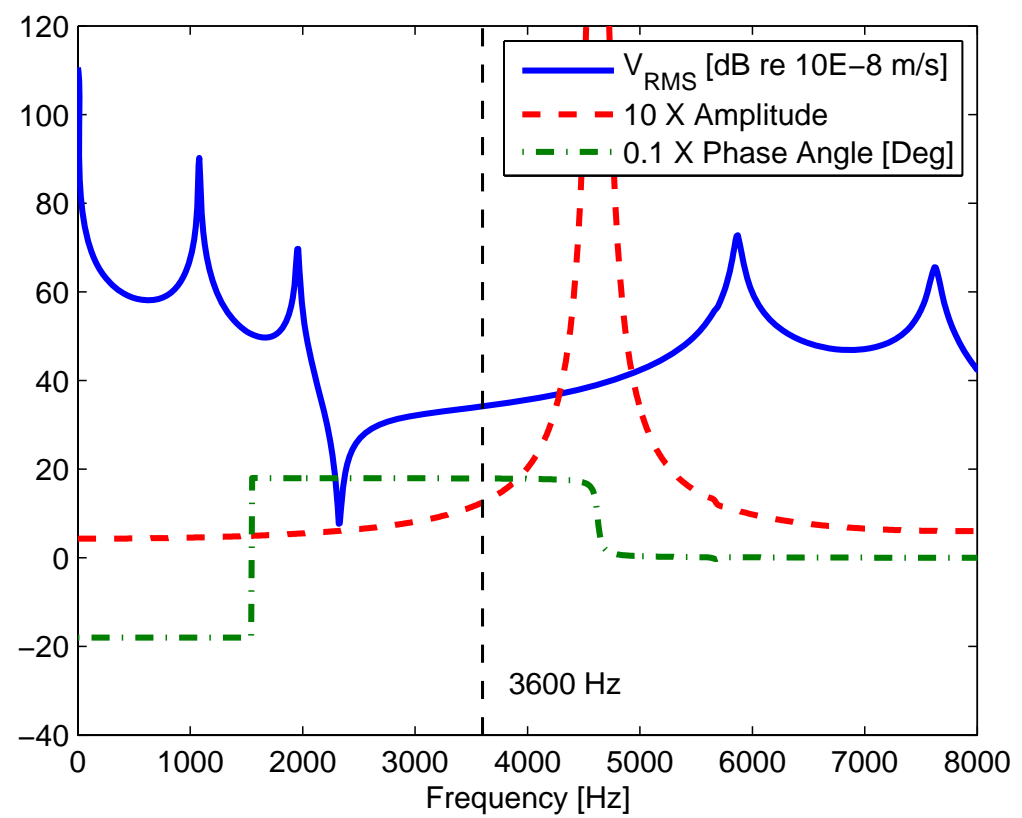

Figure 3.41: Rhombus compliant mechanism $(\mathrm{MA}=2)$ unit cell FRF 


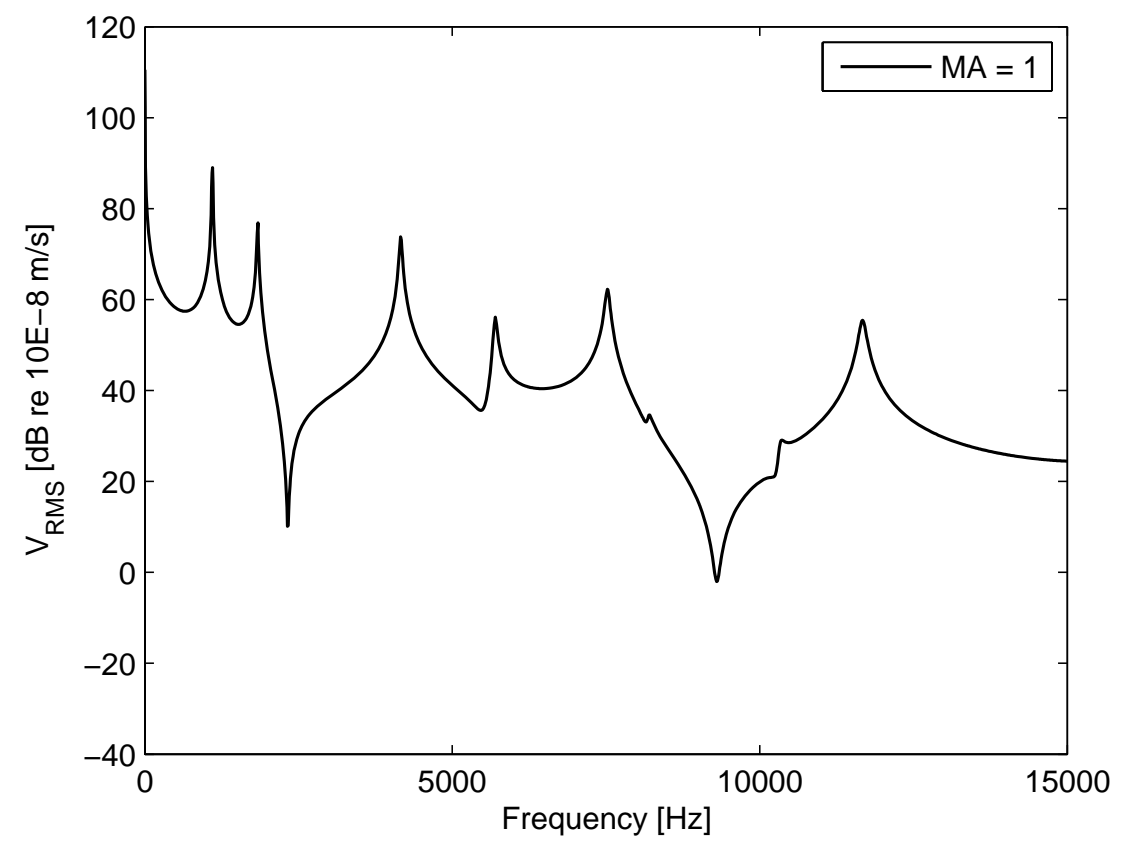

Figure 3.42: Top layer RMS velocity for rhombus compliant mechanism unit cell having $\mathrm{MA}=1$

the fundamental change that leads to this different dynamic behavior.

Results show that as mechanical advantage is reduced from 7 to 3, Figures 3.43 (a)-(c), the deformed shape and energy distribution at the first attenuation zone cutoff frequency is similar. Energy flows through core members due to a "squeezing" type motion of the unit cell, where energy content is associated with the bending of specific structural members. Examining the deformed shape and energy distribution of the unit cell with $\mathrm{MA}=2$, Figure $3.43(\mathrm{~d})$, suggests the first signs of a transition between two different types of dynamic response. Following this, the deformed shape and energy distribution for the unit cell with MA = 1, Figure 3.43 (e), exhibits a substantially different "transverse" type motion. Energy flow is associated with primarily axial deformation of specific structural members (e.g. observe the three vertical core members), and thus, it is concluded that this different dynamic behavior causes the dramatic shifting of the attenuation zone cutoff frequency seen 


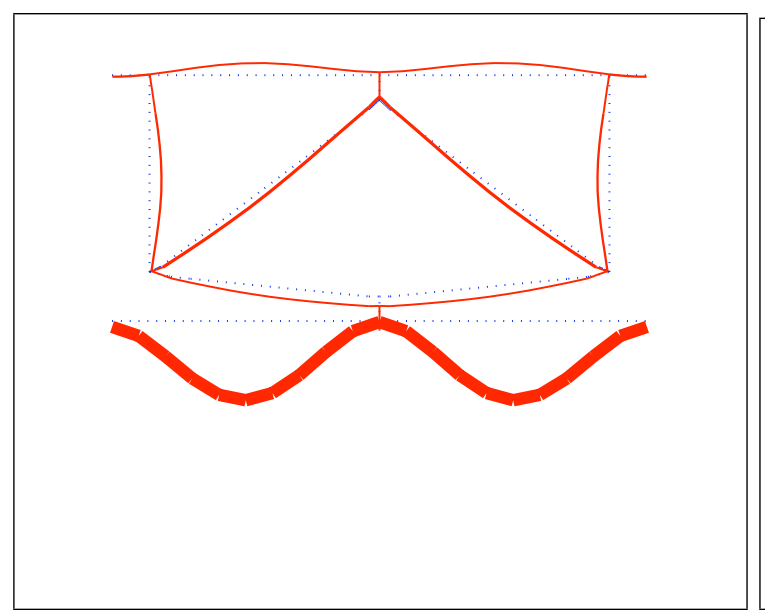

(a) $\mathrm{MA}=7$ at $5120 \mathrm{~Hz}$

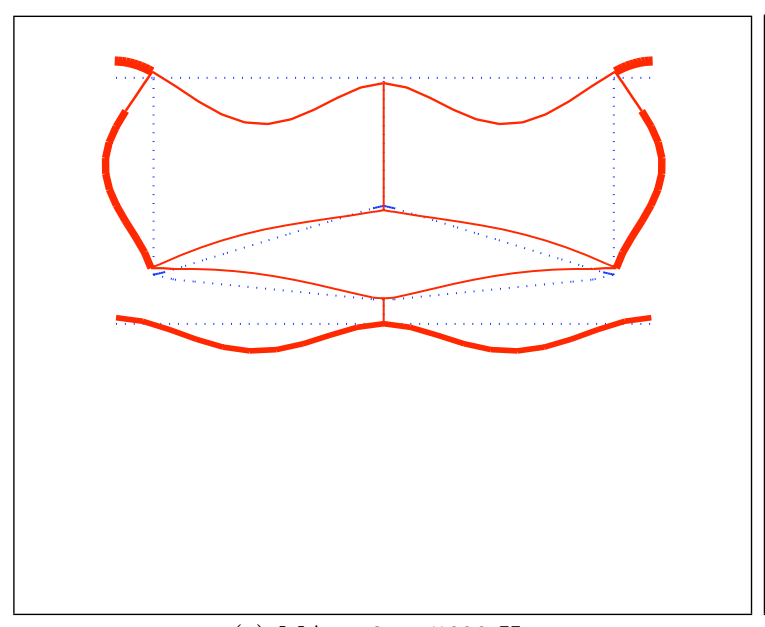

(c) $\mathrm{MA}=3$ at $5680 \mathrm{~Hz}$

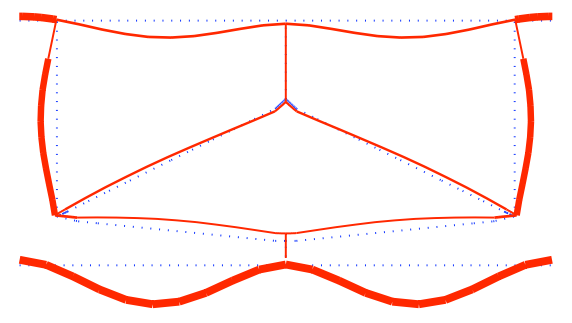

(b) $\mathrm{MA}=5$ at $5550 \mathrm{~Hz}$

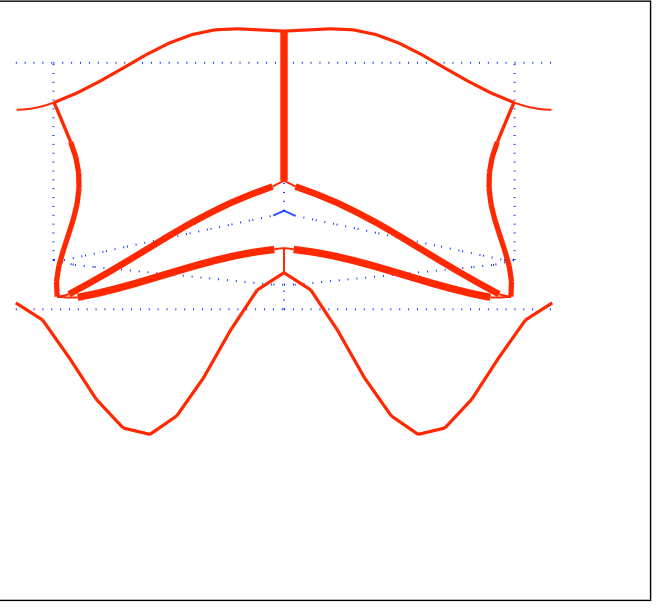

(d) $\mathrm{MA}=2$ at $5870 \mathrm{~Hz}$

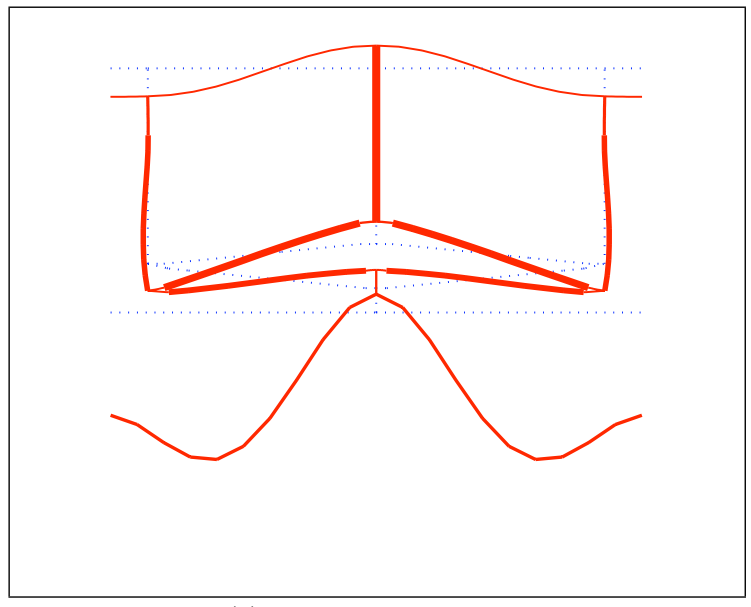

(e) $\mathrm{MA}=1$ at $4160 \mathrm{~Hz}$

Figure 3.43: Rhombus compliant mechanism unit cell deformed shape and energy distribution at the first attenuation zone cutoff frequency (dotted lines denote undeformed configuration, continuous lines denote deformed configuration, line thickness denotes energy content): (a) MA $=7$ at $5120 \mathrm{~Hz}$, (b) $\mathrm{MA}=5$ at $5550 \mathrm{~Hz}$, (c) $\mathrm{MA}=3$ at $5680 \mathrm{~Hz}$, (d) $\mathrm{MA}=2$ at $5870 \mathrm{~Hz}$, and (e) $\mathrm{MA}=1$ at 4160 
in Figure 3.42.

Thus, these results draw a clear link between mechanical advantage and resonance suppression. However, a distinction must be drawn between the effect of reducing mechanical advantage (i.e. increasing the slope of response, which leads to larger amplitudes throughout the attenuation zone), and the effect of modifying the fundamental dynamic behavior of the unit cell (i.e. transitioning from energy isolation via bending deformation to energy transmission via axial response). Furthermore, these results suggest that, in order to achieve uniform amplitude attenuation over much larger frequency ranges, a judicious choice of a compliant mechanism unit cell topology is required to maximize bending deformation while making use of large mechanical advantage.

\subsection{Unit Cell Design using Alternative Materials}

Traditional structures are typically comprised of metals such as aluminum or steel. However, the use of modern cellular composites has recently become more prevalent in the design of lightweight aerospace, automotive, and naval vehicle structures. These composites are often made of alternative materials (e.g. polymers) that, when incorporated into structures, offer comparable strength at reduced weight. Furthermore, additional benefits are gained through the use of alternative materials since the physical properties of a material can greatly affect structural dynamic response. Thus, the goal of this section is to instantiate the relationship between the material a structure with integral compliant mechanisms is made of and the resulting middle-frequency dynamic response.

A unit cell made of acrylonitrile butadiene styrene (ABS) is analyzed. ABS is an amorphous polymer that was selected in this study due to its widespread use in in- 
jection molded structures. It is important to acknowledge that the dynamic behavior of polymers can differ greatly from that of metals. In contrast with metals, both the elastic (i.e. storage) modulus, as well as, the viscous (i.e. loss) modulus of polymeric materials are typically treated as frequency dependent. Specifically, an increase in frequency has the same effect on these material physical parameters as a decrease in temperature, as extensively discussed by Sepe [89]. Equivalently stated, higher strain rates lead to a stiffer response, which is the same result obtained by decreasing the material temperature. For most polymers the glass transition temperature and the maximum damping peak in the loss modulus of the material increase about 7 degrees Celsius for every tenfold increase in frequency, per Murayama [69]. This relationship is incorporated in the analysis of a unit cell comprised of ABS.

The desired attenuation zone starting frequency in this study remains the same as that of the prior two compliant mechanism unit cell examples. The purpose of choosing the same value of $2320 \mathrm{~Hz}$ is to illustrate the connection between material selection and unit cell sizing while holding the attenuation zone starting frequency constant.

The compliant mechanism unit cell topology used in this study is the same as the first topology presented in Section 3.2.1. However, the material physical parameters for ABS are different, and they are best defined using Figure 3.44 taken from the text by Sepe [89]. In this figure $E^{\prime}$ is the elastic modulus, $E^{\prime \prime}$ is the viscous modulus, and Tan Delta $=E^{\prime \prime} / E^{\prime}$ is the loss tangent. The room temperature (i.e. 23 degrees Celsius) modulus data shown in this figure was collected using a strain rate of 1 $\mathrm{mm} / \mathrm{min}$ with a peak to peak amplitude of $1 \mathrm{~mm}$. This information is translated in a straightforward manner into an equivalent testing frequency of approximately $0.01 \mathrm{~Hz}$. Taking note that the material modulus values must be reduced by 7 degrees 


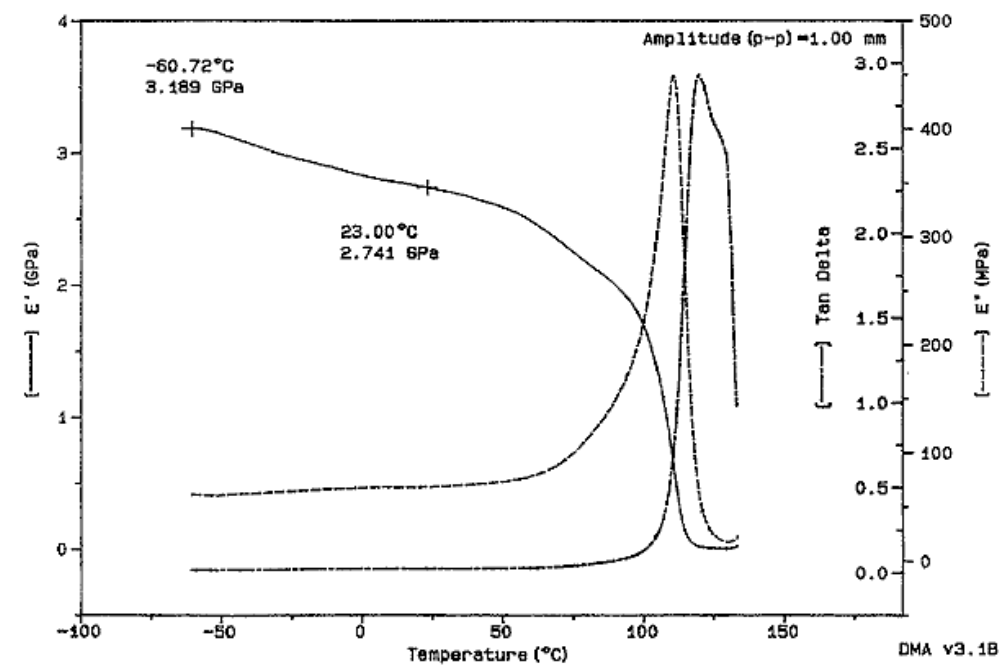

Figure 3.44: Elastic (i.e. storage) modulus, viscous (i.e. loss) modulus, and loss tangent of ABS as a function of temperature, from Sepe [89]

Celsius from room temperature for every tenfold increase in frequency, the equivalent operating temperature at $10 \mathrm{kHz}$ is approximately -19 degrees Celsius. Accordingly, the elastic modulus increases from $2.741 \mathrm{GPa}$ at $0.01 \mathrm{~Hz}$ to roughly $3.0 \mathrm{GPa}$ at $10 \mathrm{kHz}$, while the viscous modulus stays relatively constant at approximately 68.0 MPa. The initial loss factor, $\eta=0.025$, at $0.01 \mathrm{~Hz}$ is found by dividing the viscous modulus by the elastic modulus. Observe that this loss factor value is roughly 2.5 times that of aluminum at $0.01 \mathrm{~Hz}$ and that it decreases only slightly to $\eta=0.023$ or 2.3 times that of aluminum at $10 \mathrm{kHz}$. These frequency dependent relationships for the material physical parameters are straightforwardly incorporated into the unit cell computational analysis.

The width of core elements in the unit cell is taken as $1.0 \mathrm{~mm}$ compared with the $2.5 \mathrm{~mm}$ width used for the aluminum unit cell of Section 3.2.3. The width of stiffer members (e.g. ground, transmission, etc.) is taken as $2.0 \mathrm{~mm}$ versus the $5.0 \mathrm{~mm}$ width used for the unit cell in Section 3.2.3. Bottom layer element width is similarly decreased from $2.5 \mathrm{~mm}$ to $1.0 \mathrm{~mm}$. 


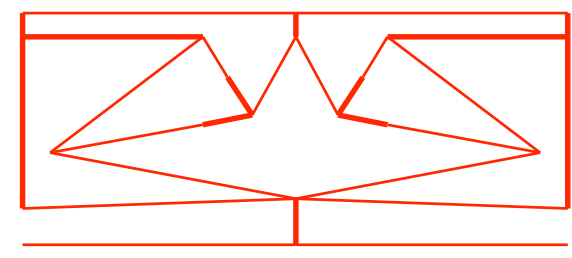

Aluminum Unit Cell

$(0.1 \mathrm{~m}$ by $0.05 \mathrm{~m})$

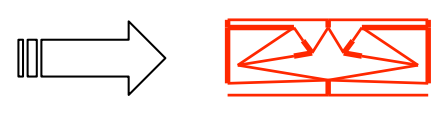

ABS Unit Cell (0.035 $\mathrm{m}$ by $0.0175 \mathrm{~m}$ )

Figure 3.45: Schematic representation of relative unit cell sizing resulting from material selection (not shown to actual scale)

By way of Eqn (3.16), a unit cell length scale, $\lambda=0.035 \mathrm{~m}$, is calculated using the appropriate unit cell parameters: the density of $\mathrm{ABS}\left(\rho=1050 \mathrm{~kg} / \mathrm{m}^{3}\right)$, the frequency dependent modulus relationships, the bottom layer element cross-section area and area moment of inertia, and an attenuation zone starting frequency of 2320 Hz. Thus, the original dimensions of $0.1 \mathrm{~m}$ by $0.05 \mathrm{~m}$ for the aluminum compliant mechanism unit cell from Section 3.2.1 are scaled down to a smaller unit cell size. For a unit cell comprised of ABS material this size is $0.035 \mathrm{~m}$ by $0.0175 \mathrm{~m}$, however, the above process illustrates that the principle can be extended to the design of a unit cell made from any arbitrary material of interest. A conceptual schematic of this principle is given in Figure 3.45.

Given these analysis parameters, the RMS transverse velocity and FRF response of the ABS unit cell was computed from $0 \mathrm{~Hz}$ to $10 \mathrm{kHz}$, evaluated in $10 \mathrm{~Hz}$ frequency steps. There are two primary features of the response shown in Figure 3.46. First, the general attenuation zone characteristics are once more confirmed over the frequency ranges of $2320 \mathrm{~Hz}$ to approximately $5000 \mathrm{~Hz}$ and 9240 to approximately $9600 \mathrm{~Hz}$. This dynamic response matches that of the aluminum unit cell shown in Figure 3.10 with the exception of the reduced sharpness of the resonant peaks. This second 


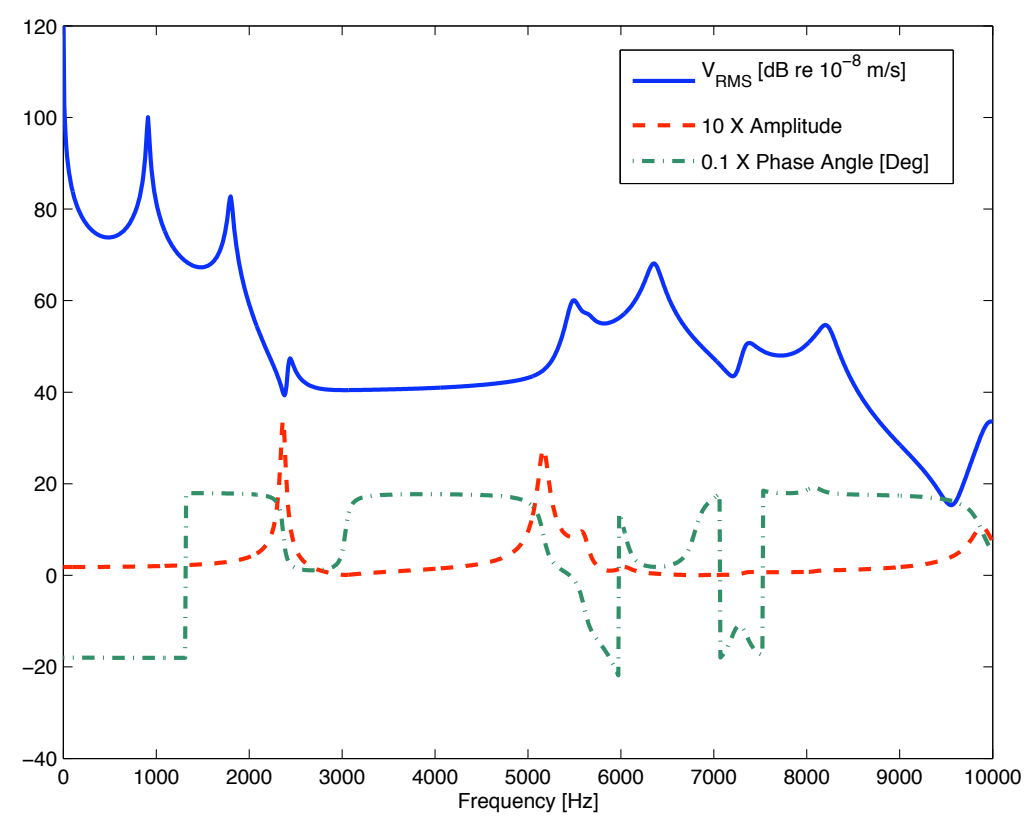

Figure 3.46: ABS compliant mechanism unit cell FRF

feature is due to the increased loss factor associated with the ABS material. Hence, in summary of this section, these results show that choosing a material that has a desired combination of physical parameters allows for specific control over structural dynamic response, plus the ability to adjust unit cell and structure sizing.

\subsection{Parallel \& Series Connected Structures}

Up to this point, the global structure configuration utilized in this dissertation consists of unit cells that are connected together in a parallel fashion. Each unit cell can be thought of as having an independent input and output. Thus, by repeatedly integrating the same unit cell into a progressively longer structure, a primarily onedimensional truss-like beam is constructed whereby the bottom layer has a multiple number of inputs and the top layer has just as many outputs.

Section 3.4.1 is focused on further investigation of parallel connected structures 
with an emphasis on handling locally applied distributed pressure loads. This section explores the connection of unit cells that have respectively different internal topologies. The objective is to reduce overall structural complexity while, at the same time, reduce vibration transmission resulting from the application of a localized pressure wave.

Sections 3.4.2 through 3.4.4 are centered on the development of series connected unit cells and their application to structures having enhanced attenuation zones, multiple attenuation zones, and multi-functional properties. Unit cells are connected in a series manner through vertical stacking. This newly introduced connection scheme expands the global one-dimensional truss-like beam structure into a twodimensional lattice structure.

In all of these sections, the compliant mechanism unit cell structures introduced thus far are used as design templates upon which these investigations are based. Slight modifications to unit cell size are implemented, as noted. In each section the underlying concept behind the study is first presented, which in turn is followed by the corresponding computational results.

\subsubsection{Parallel Connected Structures: Local Forcing}

The approach of using numerous integrated compliant mechanisms throughout the entirety of a structure has clear advantages as shown throughout Section 3.2. However, this approach leads to global structures that have greater overall geometric complexity. Depending on the nature of the applied force that a structure is subjected to, localized integral compliant mechanisms can be utilized. Take for example the two structures shown in Figures 3.47 and 3.48. In both figures a localized distributed load, spanning a total distance of $0.6 \mathrm{~m}$, is induced by an incident pressure wave, and the distributed load is shown applied to the bottom layer center of each structure. 


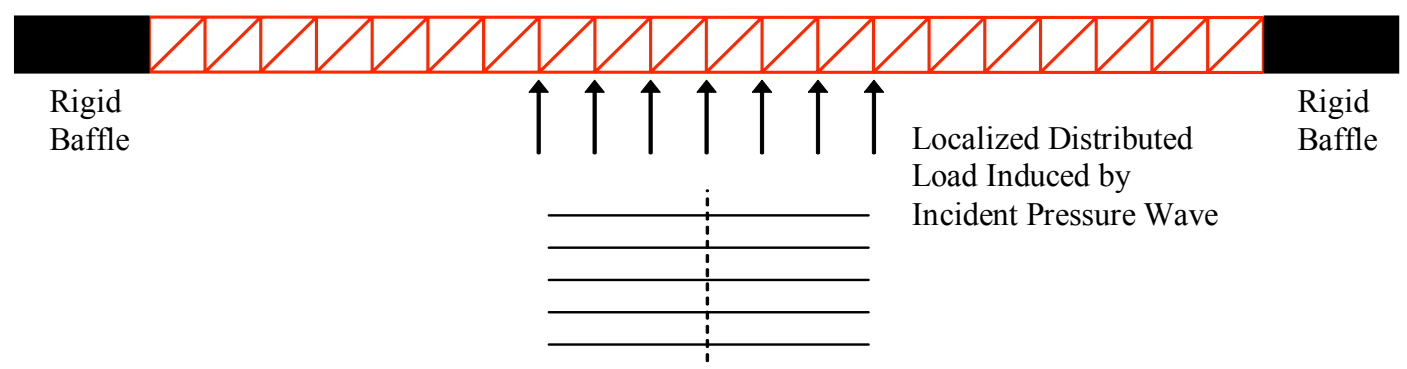

Figure 3.47: Homogeneous periodic square core structure subjected to a localized distributed load

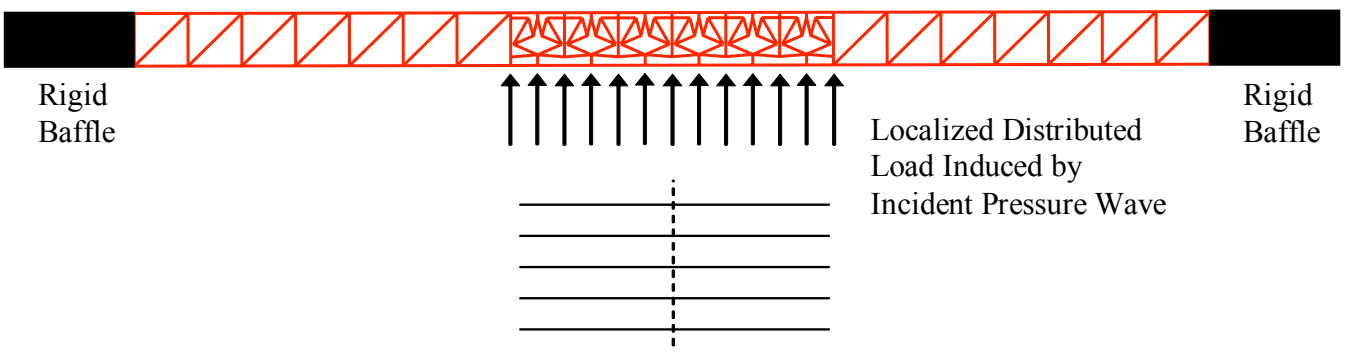

Figure 3.48: Heterogeneous structure comprised of square core unit cells connected in parallel with compliant mechanism units cells and subjected to a localized distributed load 
This locally applied force can be thought of as representative of a confined excitation source such as a surface mounted machine (e.g. a blower or compressor).

In Figure 3.47 the structure can be considered globally homogeneous meaning that all unit cells have a square core topology identical to the one from Section 3.1. Alternatively, in Figure 3.48 a globally heterogeneous structure is shown. Only the unit cells directly subjected to the locally applied distributed load are assigned a compliant mechanism topology. Observe that the compliant mechanism topology from Section 3.2.1 is used, and that this approach to integrating compliant mechanisms into a global structure minimizes overall geometric complexity. Accordingly, beyond the edges of the confined loading, square core unit cells are connected in parallel with compliant mechanism unit cells as illustrated in Figure 3.48.

The same material physical parameters, unit cell sizing, and analysis parameters utilized for the square core unit cell (Section 3.1) and the first compliant mechanism unit cell (Sections 3.2.1-3.2.3) are used. In Figure 3.49 the RMS normal velocity response of the top layer of each structure is shown computed from $0 \mathrm{~Hz}$ to $5 \mathrm{kHz}$, evaluated at $10 \mathrm{~Hz}$ frequency intervals. The expected peak at $3870 \mathrm{~Hz}$ is visible in the response of the homogeneous structure. Considering an excitation source that operates at or near this frequency, it is logical to expect large levels of structural vibration, and hence, transmitted sound from the homogenous structure. In contrast, the response of the heterogeneous structure shows that this peak is significantly reduced by an average of approximately $13 \mathrm{~dB}$ over a frequency range from $3500 \mathrm{~Hz}$ to $4000 \mathrm{~Hz}$.

Despite improved performance at frequencies centered about $3870 \mathrm{~Hz}$, limitations of this method exist as evidenced by the significant resonances of the heterogeneous structure that have been newly introduced near $900 \mathrm{~Hz}, 1800 \mathrm{~Hz}$, and $5 \mathrm{kHz}$. Thus, 


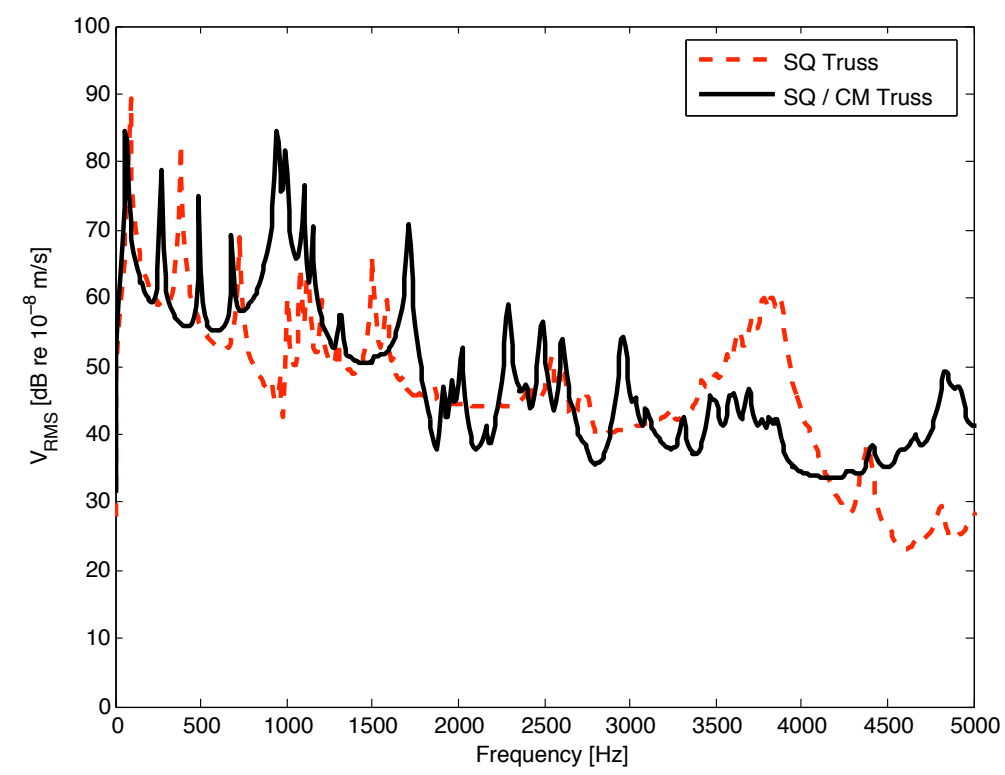

Figure 3.49: Top layer RMS velocity of square core structure subjected to localized forcing versus a structure comprised of square core unit cells connected in parallel with compliant mechanism units cells and subjected to the same localized forcing

caution must be exercised to completely understand the vibratory problem of interest. Avoiding dwell time close to these frequencies during operation ramp up of the localized source is important since these resonances represent an increase in structural vibration when compared to the response of the original homogeneous structure.

\subsubsection{Series Connected Structures: Enhanced Attenuation Zones}

In this section series connected structures that have a greater number of compliant mechanism layers are examined. Unit cells are connected in series fashion to determine whether increased vibration attenuation is achievable. The rhombus compliant mechanism unit cell (Sections 3.2.4-3.2.5) is utilized. With the exception of the thicker members of the unit cell, all material physical parameters, unit cell sizing, and analysis parameters are treated the same. The thicker members of the 


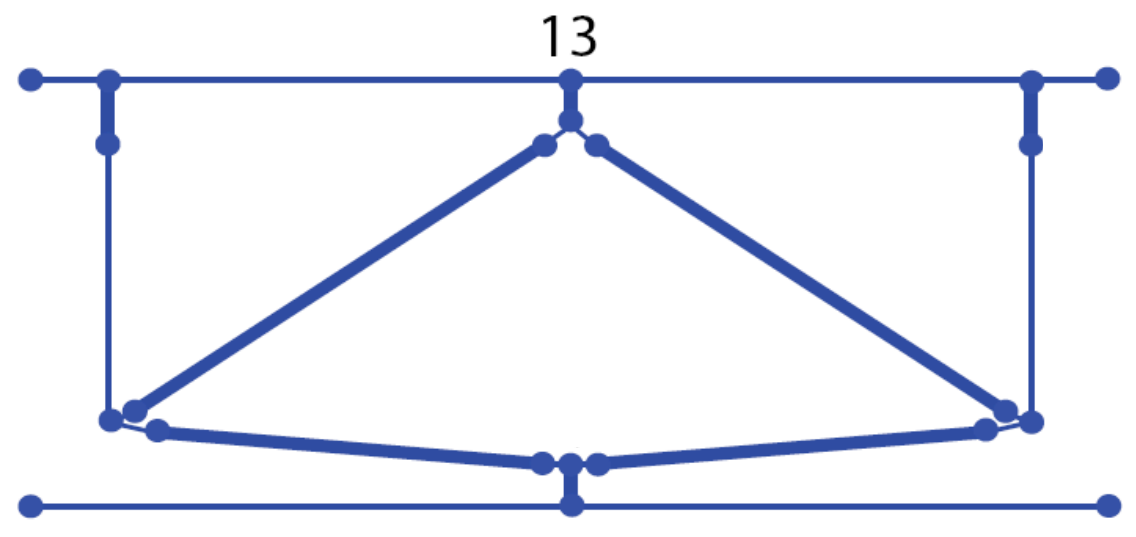

Figure 3.50: Rhombus compliant mechanism unit cell model (the number denotes the top layer middle node)

unit cell shown in Figure 3.50 are assigned an increased width of $7.5 \mathrm{~mm}$. This thickness value raises the stiffness of the compliant mechanism core and extends the first attenuation zone cutoff frequency by approximately $500 \mathrm{~Hz}$ when compared with the original rhombus compliant mechanism unit cell response in Figure 3.28.

The investigation entails the incorporation of the rhombus compliant mechanism unit cells into increasingly taller serial structures. Focusing on the first attenuation zone frequency range, structural dynamic response is computed from $0 \mathrm{~Hz}$ to $8 \mathrm{kHz}$, evaluated in $10 \mathrm{~Hz}$ frequency steps for each structure. The structures range in height from one unit cell (i.e. $0.05 \mathrm{~m})$ to 20 unit cells $(1.0 \mathrm{~m})$. The physical layout of each rhombus compliant mechanism series structure is given in Figure 3.51. Observe that as unit cells are stacked, the top layer of the preceding unit cell becomes the bottom layer of the following unit cell. Likewise, the output of a lower unit cell becomes the input of higher unit cell.

The response of each structure is given in Figure 3.52. It is evident that each unit cell that is added in series reduces response within the first attenuation zone by approximately $15 \mathrm{~dB}$. This downward trend continues until a point at which the 


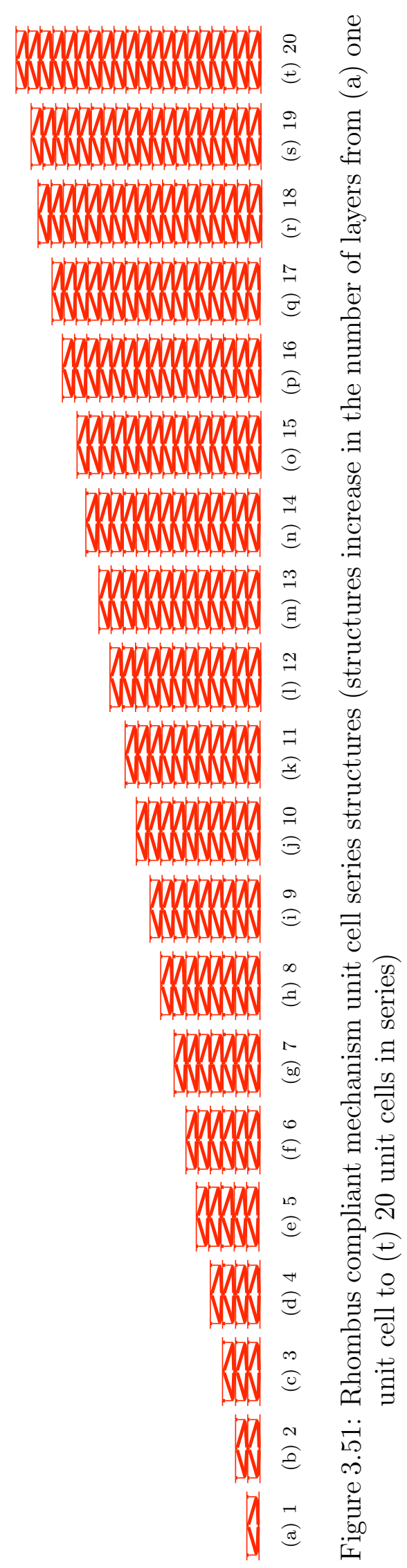




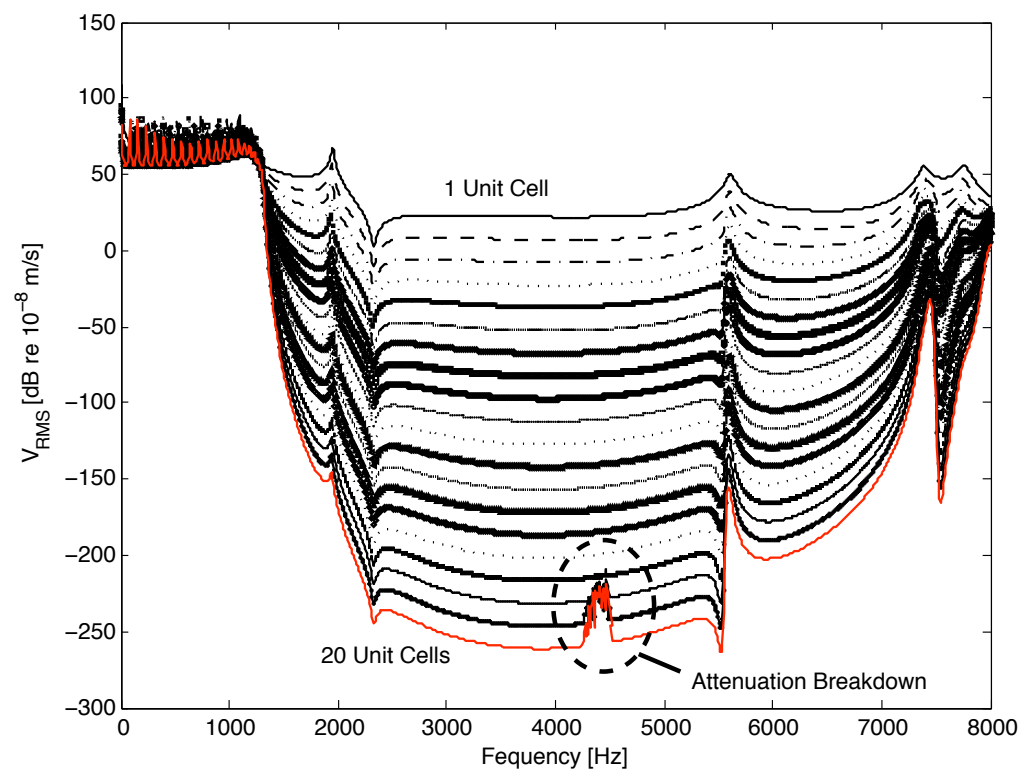

Figure 3.52: Top layer RMS velocity of rhombus compliant mechanism series structures

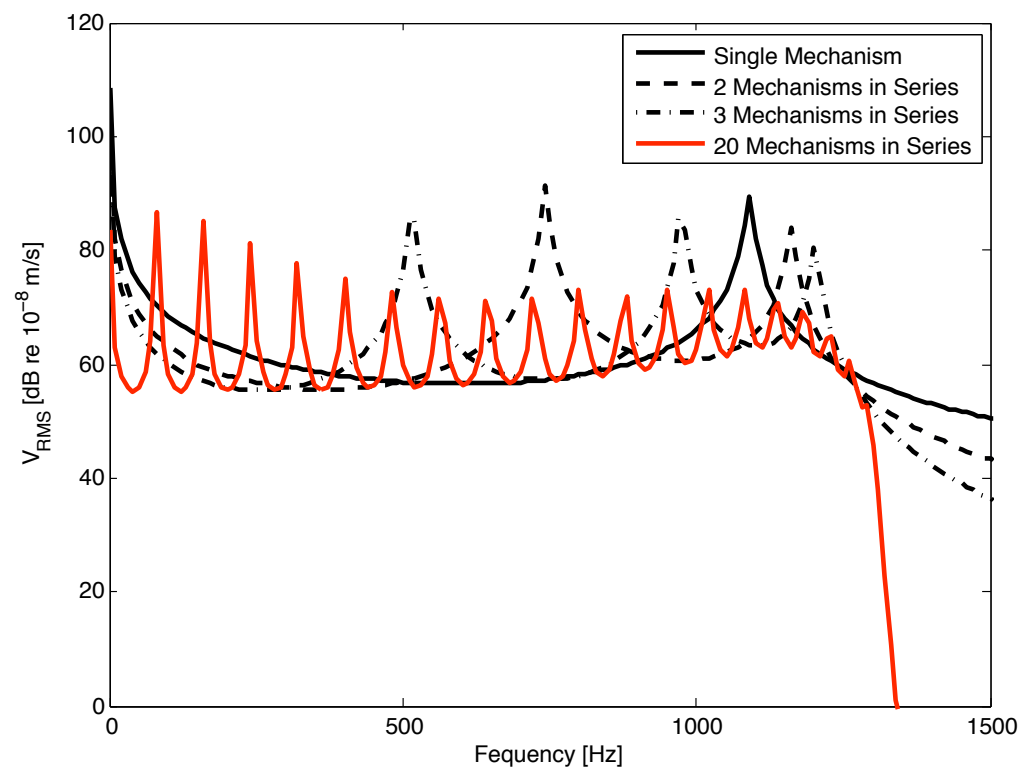

Figure 3.53: Top layer RMS velocity of rhombus compliant mechanism series structures from $0 \mathrm{~Hz}$ to $1500 \mathrm{~Hz}$ 


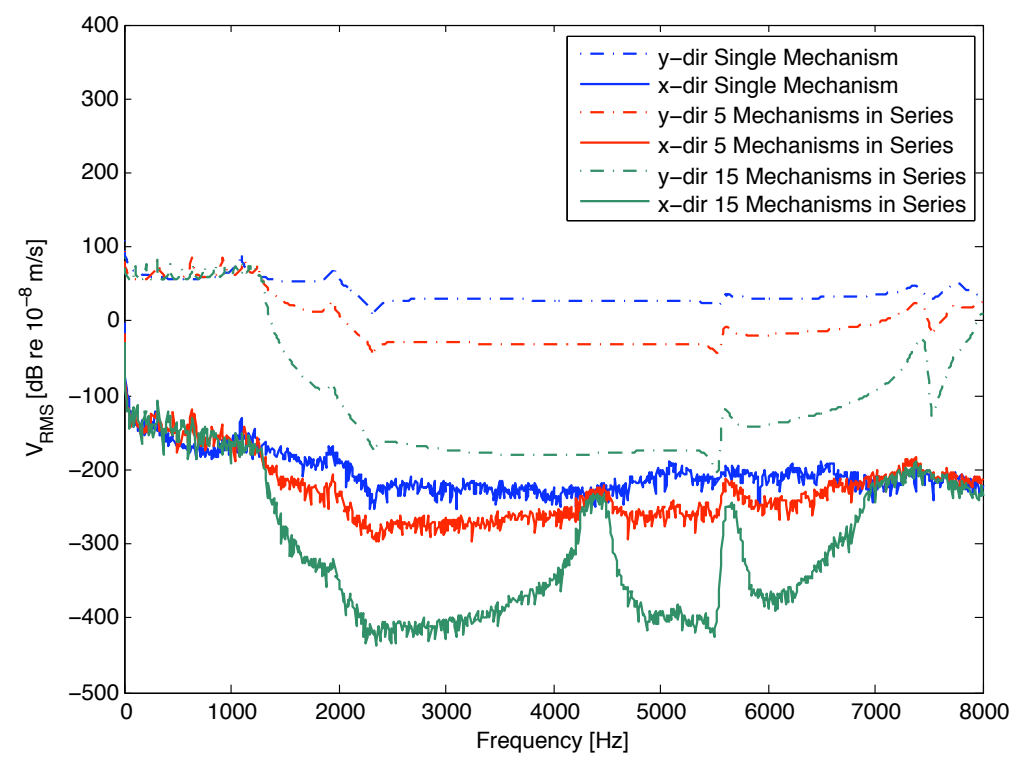

Figure 3.54: Top layer transverse and horizontal RMS velocity of rhombus compliant mechanism series structures

attenuation zone begins to break down as highlighted in this figure. The discussion of this phenomenon is deferred for the moment.

However, also of interest is the behavior of these series structures at frequencies below $1500 \mathrm{~Hz}$. As additional unit cells are connected in series, the number of resonances within this lower frequency band conjointly increases, as illustrated in Figure 3.53. For clarity in this figure the response of the standalone unit cell is shown along with the response of the structures having two, three, and 20 unit cells connected in series.

The breakdown in the attenuation zone mentioned above in relation to Figure 3.52 occurs for structures having 16 or more compliant mechanism unit cells connected in series. The cause of this breakdown is explored by computing, not only the top layer RMS transverse velocity, but also the top layer middle node (e.g. node 13 in Figure 3.50) RMS horizontal velocity of several series structures. The structural 


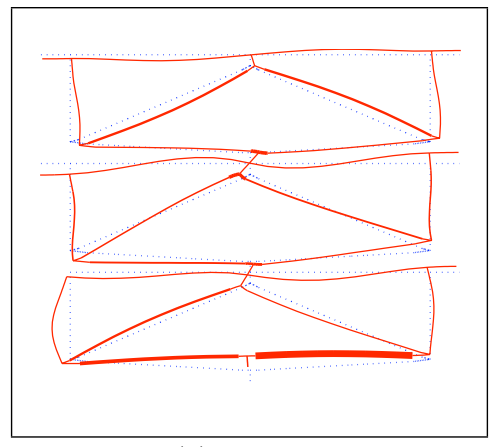

(a) $4410 \mathrm{~Hz}$

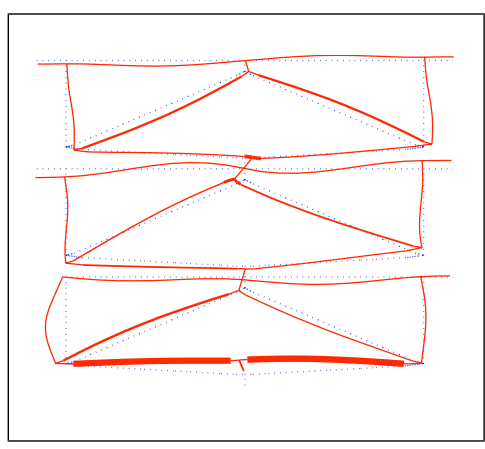

(b) $4440 \mathrm{~Hz}$

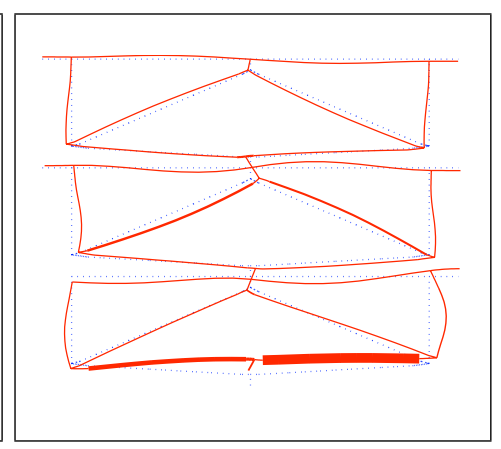

(c) $4480 \mathrm{~Hz}$

Figure 3.55: Energy distribution in the top three rhombus compliant mechanism unit cells for the structure with 20 unit cells in series: (a) $4410 \mathrm{~Hz}$, (b) 4440 $\mathrm{Hz}$, and (c) $4480 \mathrm{~Hz}$ (dotted lines denote undeformed configuration, continuous lines denote deformed configuration)

dynamic response of the single compliant mechanism unit cell is compared with that of structures having five and 15 unit cells connected in series, respectively, in Figure 3.54.

This figure shows that as the number of unit cells in series increases above 15 the RMS horizontal velocity amplitude begins to approach the RMS transverse velocity amplitude in the frequency range of 4200-4600 Hz. This phenomenon leads to a crosscoupling of the horizontal and transverse velocity of the structure, and hence, to the breakdown in the attenuation zone highlighted in Figure 3.52. To better understand this behavior, the total energy of the structure having 20 unit cells connected in series was computed. The deformed shape and energy distribution in the top three unit cells of this structure are shown in Figures 3.55 (a), (b), and (c) at frequencies within the 4200-4600 Hz range. The dynamic response at the top of the structure is characterized by side to side movement and energy flow into the core of each unit cell. These resonant modes are related to the cross coupling of the horizontal and transverse velocities.

Within a broad context, it is reasonable to assume that the results presented 
in this section can be generalized to any compliant mechanism unit cell topology connected in series. First, unit cells can be connected in series to achieve greater attenuation within a predefined frequency range. Second, the additive effect of a greater number of unit cells connected in series is limited due to the specific behavior of the structure. This specific behavior is logically connected to the topological details of the structure and should generally vary depending on the selected topology for the compliant mechanism unit cell. As further unit cells are stacked, local resonances can occur to limit the effectiveness of the series structure. Accordingly, while significant attenuation can be achieved, the attenuation does not increase without bound.

\subsubsection{Series Connected Structures: Multiple Attenuation Zones}

The next phase in the study of series connected structures is to consider a composite unit cell comprised of two distinctly sized unit cells, as shown in Figure 3.56. Each individual unit cell is considered to have a response attenuation zone that spans a unique frequency range. The goal here is to better understand the influence of the standalone response of each unit cell on the additive composite response of the series structure. The compliant mechanism unit cell topology, ABS material physical parameters, and analysis parameters from Section 3.3 are utilized for both unit cells.

The sizing, and consequently the stiffness, of various members within each unit cell is manually adjusted such that different attenuation zone frequency ranges are achievable. The bottom unit cell is assigned the same member widths as the unit cell defined in Section 3.3. Several additional core members of the top unit cell are assigned the thicker width of $2.0 \mathrm{~mm}$. In Figure 3.56 thinner lines denote members having a width of $1.0 \mathrm{~mm}$ and thicker lines denote members having a width of 2.0 $\mathrm{mm}$.

The RMS transverse velocity response of the top unit cell, the bottom unit cell, 


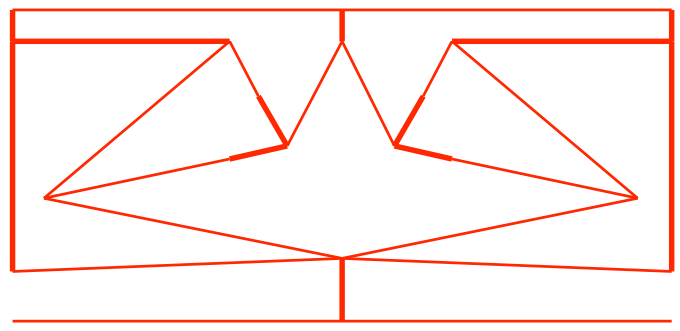

(a) Bottom unit cell

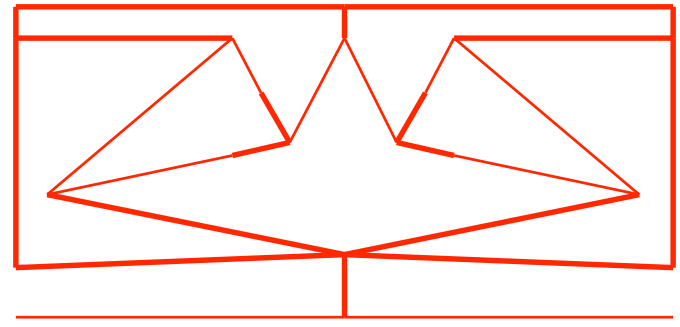

(b) Top unit cell

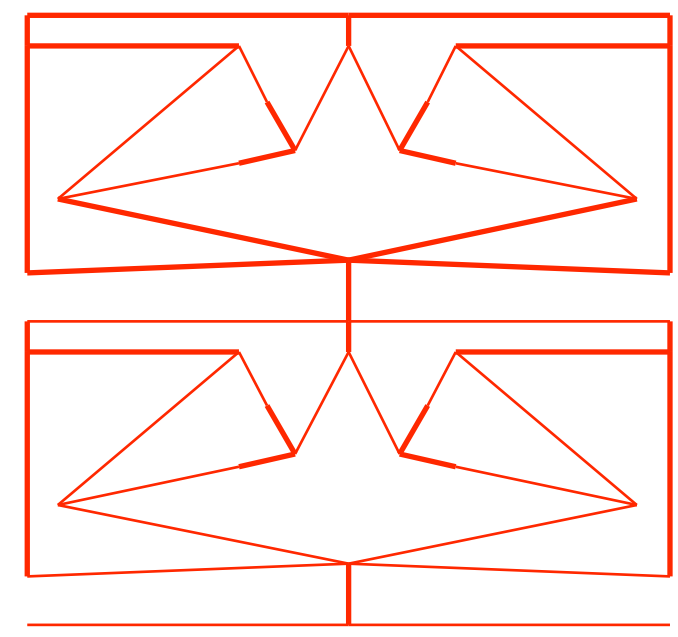

(c) Series compliant mechanism structure

Figure 3.56: Two unit cells and their assembly into a series structure: (a) bottom unit cell, (b) top unit cell, and (c) series compliant mechanism structure (thicker lines denote members with a width of $2.0 \mathrm{~mm}$, thinner lines denote members with a width of $1.0 \mathrm{~mm}$ )

and the series structure was respectively computed from $0 \mathrm{~Hz}$ to $10 \mathrm{kHz}$ at $10 \mathrm{~Hz}$ frequency increments. The respective standalone and composite structural dynamic responses of all three are given in Figure 3.57. The dynamic response of the bottom unit cell is a repeat of the response shown in Figure 3.46. The dynamic response of the top unit cell exhibits a shift towards higher frequencies across the $10 \mathrm{kHz}$ frequency range relative to that of the bottom unit cell. This shift is a consequence of the increased width of the unit cell core members and the resulting increased stiffness. Below $2 \mathrm{kHz}$ a single unit cell resonance is visible in the response of the top unit cell. 


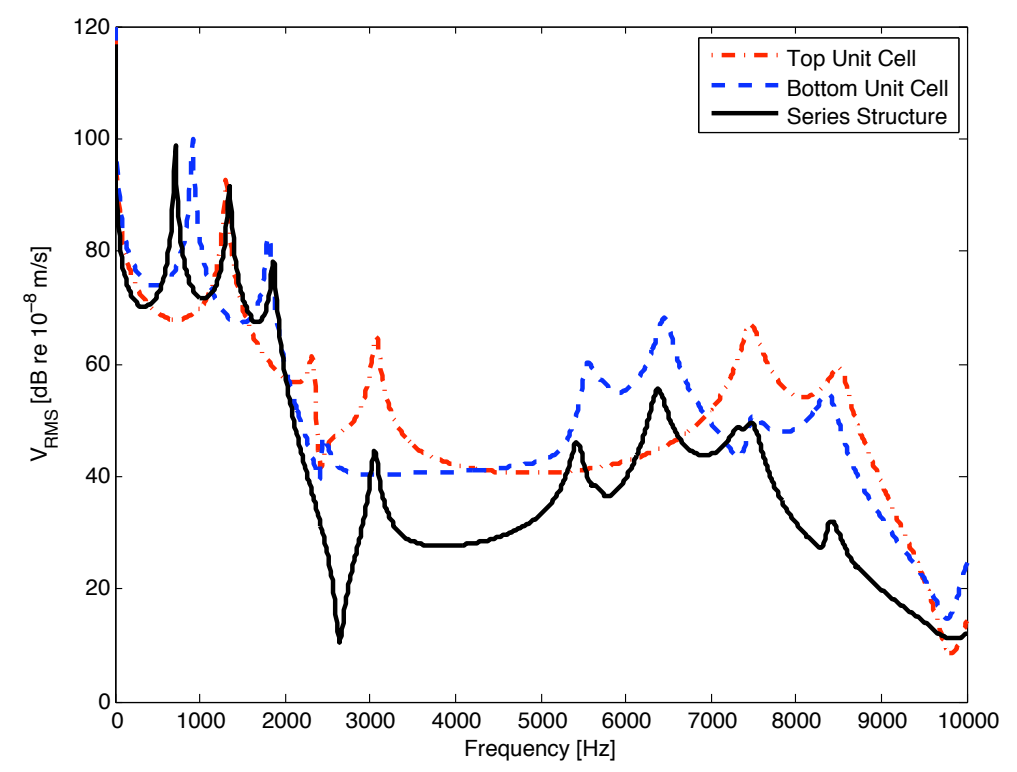

Figure 3.57: Top layer RMS velocity of bottom unit cell, top unit cell, and series compliant mechanism structure

The first attenuation zone is also evident from $2320 \mathrm{~Hz}$ to approximately $6500 \mathrm{~Hz}$ with greater modal density at the beginning of the zone. The resonances that occur in the response of the bottom unit cell from $5500-8500 \mathrm{~Hz}$ are confined to a smaller frequency range of $7000-8500 \mathrm{~Hz}$ for the top unit cell. The second attenuation zone from $9240-9600 \mathrm{~Hz}$ is visible in the dynamic response of both individual unit cells.

Moving to the dynamic response of the series structure, the separate unit cell response characteristics are combined to describe the response of the series structure, i.e. all resonant peaks are present. Alternatively stated, the response of the series structure appears as a superposition of the individual unit cell responses. Below 2 $\mathrm{kHz}$ three resonances now appear. Above $2 \mathrm{kHz}$ and within the frequency ranges in which the attenuation zones overlap, RMS transverse velocity levels are reduced. Above $5 \mathrm{kHz}$ four peaks are present, which respectively represent the peaks found in the dynamic response of the individual top and bottom unit cells. On average, 
the dynamic response of the series structure from $2320 \mathrm{~Hz}$ to $10 \mathrm{kHz}$ is reduced by approximately $15 \mathrm{~dB}$ relative to either of the standalone unit cell responses.

The reduction in structural dynamic response achieved using unit cells in series is constrained by the fact that the stacking of unit cells introduces increased modal density. This increased modal density is a consequence of the superposition of the responses of the individual unit cells. Despite this limitation, at frequencies beyond the first attenuation zone, the amplitude of all resonances is reduced.

\subsubsection{Series Connected Structures: Introduction to Multi-Functional Structures}

In this final section dealing with series connected structures, two configurations are examined at the unit cell and structure level. The first configuration consists of two square core unit cells connected in series (from here on referred to as a "double layer square core" unit cell and structure). The second configuration consists of a square core unit cell connected in series to a compliant mechanism unit cell (from here on referred to as a "multi-functional" unit cell and structure). The objective of this investigation is to introduce multi-functional structures that are capable of supporting static loads while having enhanced dynamic performance. Thus, in the case of the multi-functional configuration, the square core unit cell can be thought of as the structural component, and the compliant mechanism unit cell can be thought of as the portion of the structure designed for specific dynamic functionality. The performance of the multi-functional unit cell, as it pertains to vibration transmission, is compared with the performance of the purely structural double layer square core unit cell.

The conceptual model for the double layer square core unit cell incorporates the standalone square core unit cell from Section 3.1 as a design template for both the top 
and bottom unit cells shown in Figures 3.58 (a) and (b), respectively. All material physical parameters and analysis parameters are assumed to be the same for both individual unit cells, except for unit cell member sizing. The width of members in the bottom unit cell is the same as the standalone square core unit cell in Section 3.1 with the exclusion of the top facesheet, which is assigned a thickness of $2.5 \mathrm{~mm}$ instead of $5.0 \mathrm{~mm}$, Figure 3.58 (a). Consequently, the top unit cell must also be assigned a bottom facesheet thickness of $2.5 \mathrm{~mm}$, Figure 3.58 (b). The conceptual model of the resultant series structure is shown graphically in Figure 3.58 (c). A double layer square core structure composed of six double layer square core unit cells is given in Figure 3.59.

The conceptual model for the multi-functional unit cell also incorporates the same standalone square core unit cell from Section 3.1 as a design template for the bottom unit cell shown in Figure 3.60 (a). However, the top unit cell in Figure 3.60 (b) is modeled after the standalone compliant mechanism unit cell from Section 3.2.1. Again, all material physical parameters and analysis parameters are assumed to be the same for both individual unit cells, except for unit cell member sizing. The bottom unit cell is once more assigned a top facesheet thickness of $2.5 \mathrm{~mm}$. For simplicity, all members of the top unit cell are assigned a thickness of $2.5 \mathrm{~mm}$. The conceptual model of the resultant series structure is provided in Figure 3.60 (c). A structure assembled from six multi-functional unit cells is shown in Figure 3.61. 


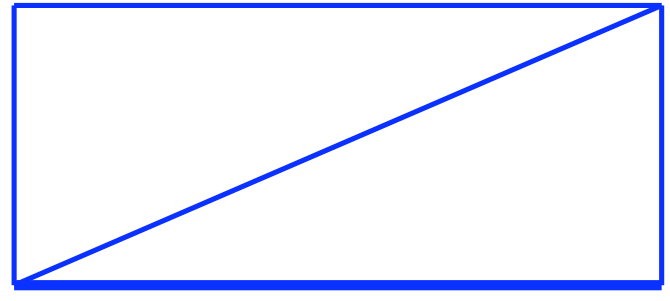

(a) Bottom unit cell

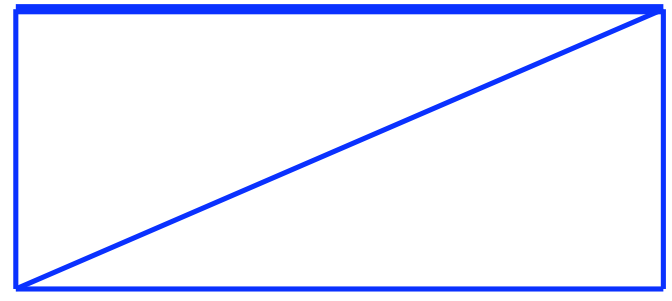

(b) Top unit cell

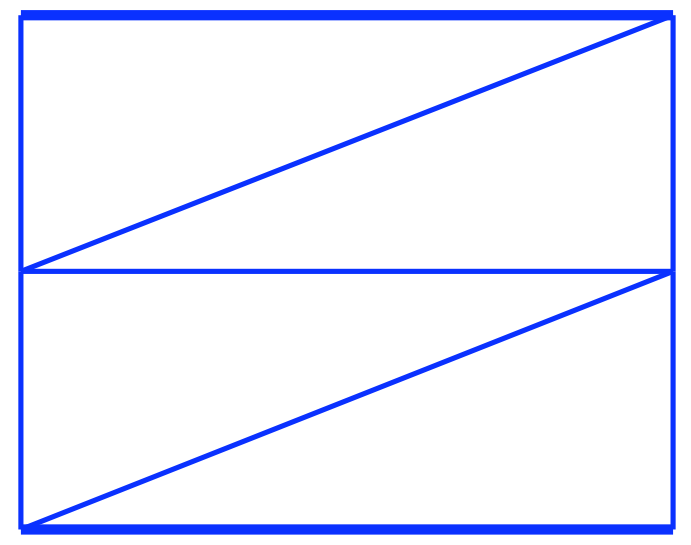

(c) Double layer square core unit cell

Figure 3.58: Two individual unit cells and their assembly into a series structure: (a) bottom unit cell, (b) top unit cell, and (c) double layer square core unit cell (thicker lines denote members with a width of $5.0 \mathrm{~mm}$, thinner lines denote members with a width of $2.5 \mathrm{~mm}$ )

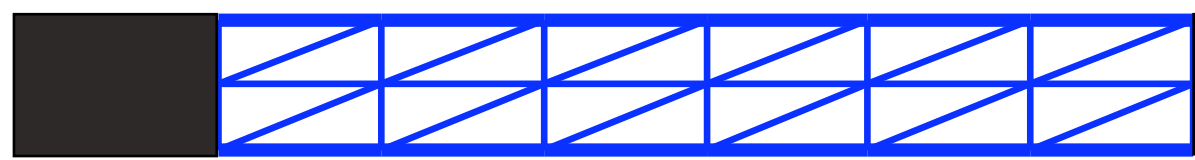

Rigid

Rigid

Baffle

Baffle

Figure 3.59: Periodic structure comprising six double layer square core unit cells 


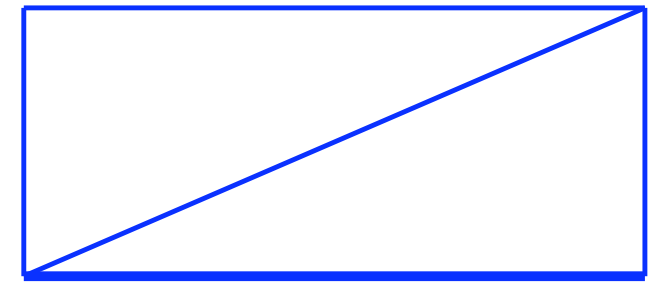

(a) Bottom unit cell

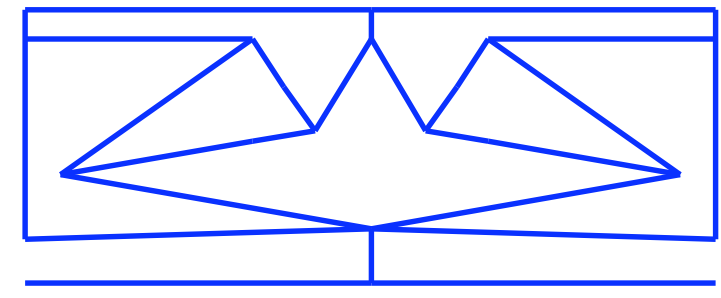

(b) Top unit cell

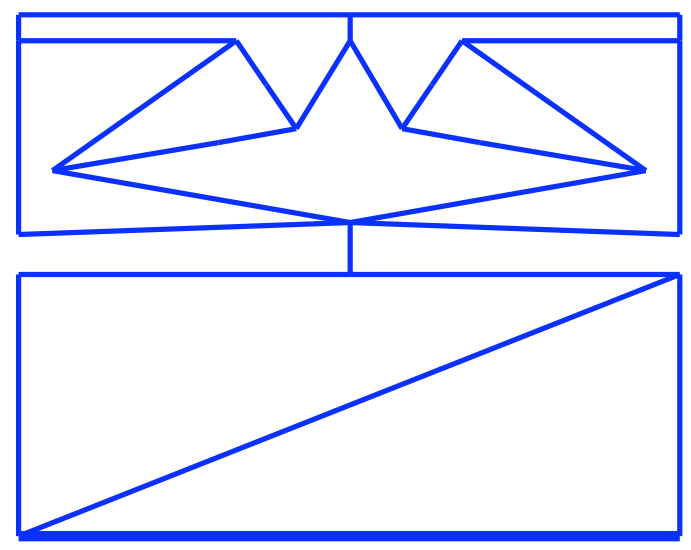

(c) Multi-functional unit cell

Figure 3.60: Two individual unit cells and their assembly into a series structure: (a) bottom unit cell, (b) top unit cell, and (c) multi-functional unit cell (thicker lines denote members with a width of $5.0 \mathrm{~mm}$, thinner lines denote members with a width of $2.5 \mathrm{~mm}$ )

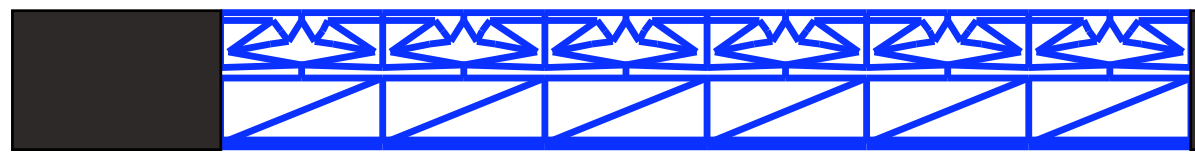

Rigid

Rigid

Baffle

Baffle

Figure 3.61: Periodic structure comprising six multi-functional unit cells 


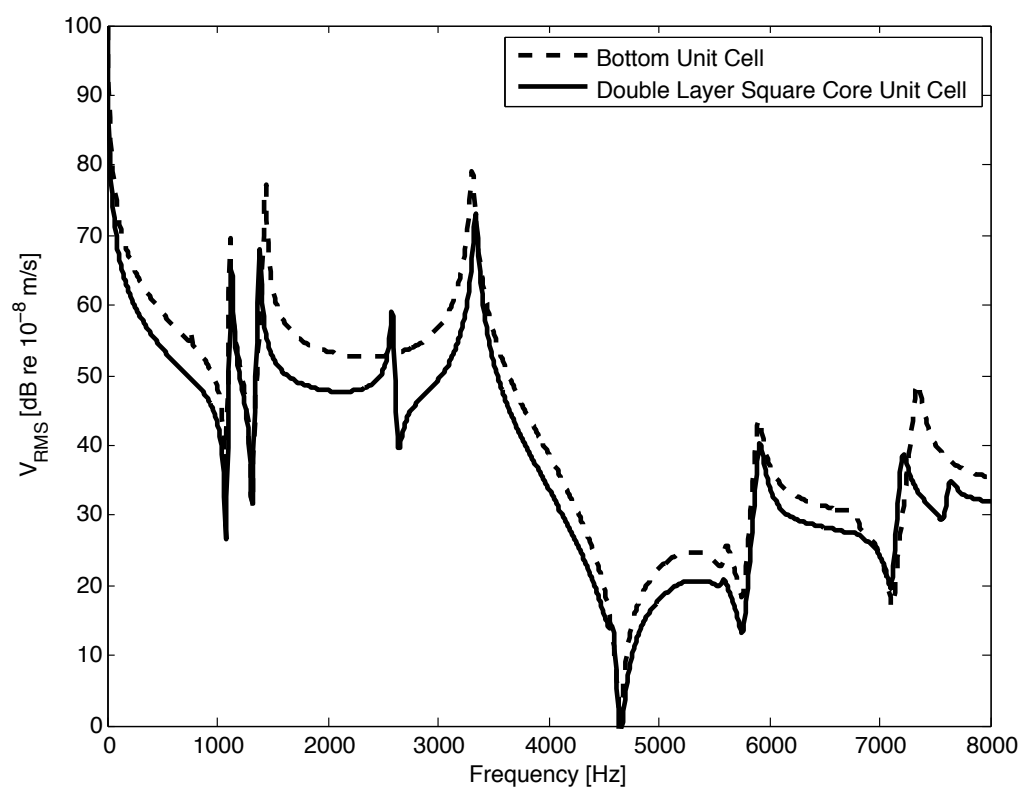

Figure 3.62: Top layer RMS velocity of bottom unit cell and double layer square core unit cell

Several analyses were performed to characterize the various unit cells and structures that were just defined. All of the top layer RMS transverse velocity response results shown in the figures that follow focus on the first attenuation zone frequency range. Thus, structural dynamic response was computed from $0 \mathrm{~Hz}$ to $8 \mathrm{kHz}$, evaluated in $10 \mathrm{~Hz}$ frequency steps.

A comparison is made in Figure 3.62 between the bottom unit cell from Figure 3.58 (a) and the double layer square core unit cell from Figure 3.58 (c). The response of the bottom unit cell is a predictor of the response of the double layer square core unit cell with comparable amplitude throughout the frequency range. Observe that changing the thickness of the top facesheet of the bottom unit cell splits the original square core unit cell resonant peak at $1120 \mathrm{~Hz}$, Figure 3.3 (a), into two peaks at $1130 \mathrm{~Hz}$ and $1390 \mathrm{~Hz}$, Figure 3.62. Furthermore, the unit cell resonance at $3700 \mathrm{~Hz}$, Figure 3.3 (a), moves to $3300 \mathrm{~Hz}$, Figure 3.62, as a result of the reduced 


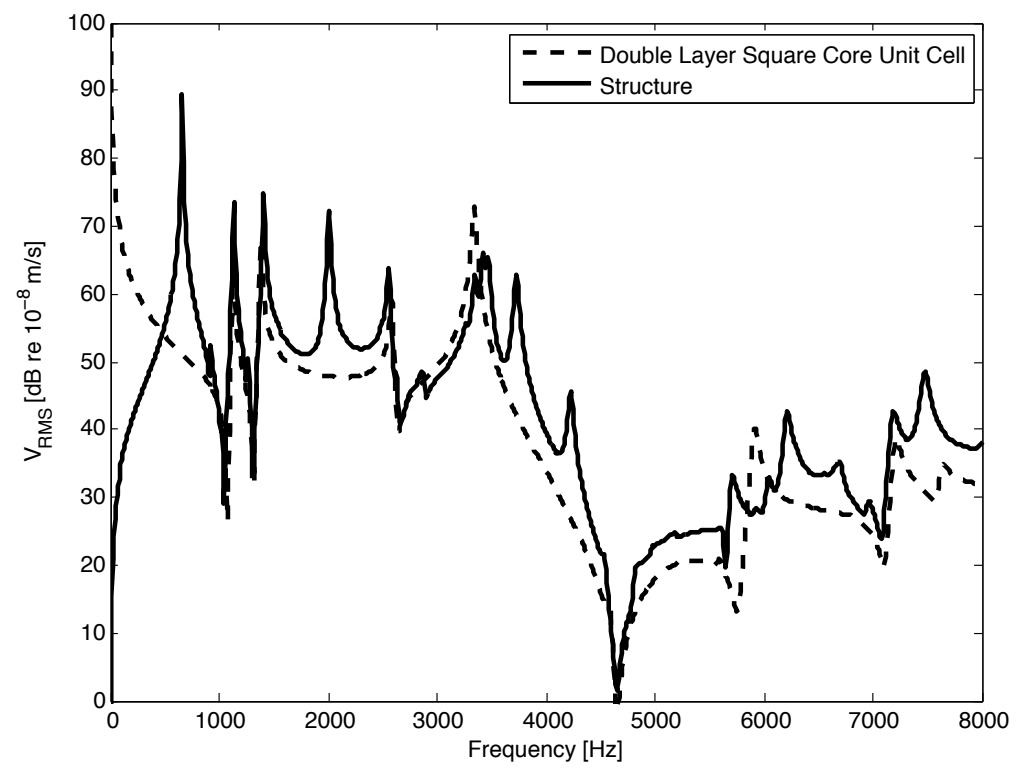

Figure 3.63: Top layer RMS velocity of double layer square core unit cell and structure

stiffness of the unit cell.

In Figure 3.63, the top layer RMS transverse velocity response of the double layer square core unit cell from Figure 3.58 (c) is compared with the response of the structure from Figure 3.59. The first resonant mode of the structure occurs at 660 Hz. Increased modal density between $1100-2600 \mathrm{~Hz}$ and $3000-4500 \mathrm{~Hz}$ is also visible in the response of the structure. Nonetheless, the response of the double layer square core unit cell predicts the global structural dynamic behavior.

The top layer RMS transverse velocity response of the multi-functional unit cell from Figure 3.60 (c) and each of its individual unit cell constituents now is examined. Figure 3.64 shows the now familiar response of the bottom (i.e. square core) unit cell from Figure 3.60 (a) having the same notable resonant peak at $3300 \mathrm{~Hz}$. The top (i.e. compliant mechanism) unit cell from Figure 3.60 (b) exhibits an attenuation zone that spans the frequency range of $2320 \mathrm{~Hz}$ to $5000 \mathrm{~Hz}$ with greater amplitude 
of response respectively before and after these two frequencies. Combining these two individual unit cells into a multi-functional unit cell results in a dynamic response that shares the resonant characteristics of both individual unit cells, per the results from Section 3.4.3. However the resonant peak at $3300 \mathrm{~Hz}$ is significantly reduced. This $25 \mathrm{~dB}$ reduction is a consequence of the unit cell series coupling, and it illustrates the application of this method in building structures with multi-functional capabilities. In Figure 3.65, the top layer RMS transverse velocity response of the multi-functional unit cell from Figure 3.60 (c) is compared with the response of the structure from Figure 3.61. The unit cell response once more accurately predicts the structure response, despite the presence of greater modal density.

Finally, the response of the double layer square core unit cell is compared with the response of the multi-functional unit cell in Figure 3.66. The structural dynamic response of the double layer square core structure is compared with that of the multifunctional structure in Figure 3.67. A reduction of approximately $14 \mathrm{~dB}$ is seen in the response of the multi-functional structure when compared to the response of the double layer square core structure over the frequency range of 2320-5000 Hz.

To summarize, the bottom layer of the multi-functional structure can be considered a load bearing member whose dynamic characteristics generally dominate structural dynamic performance. In contrast, the top layer of the multi-functional structure can be considered as an acoustic lining that reduces the amplitude of these dominant dynamic characteristics within a prescribed response frequency range. These results suggest promising applications of this research to the solution of practical engineering problems concerned with structural-borne noise. 


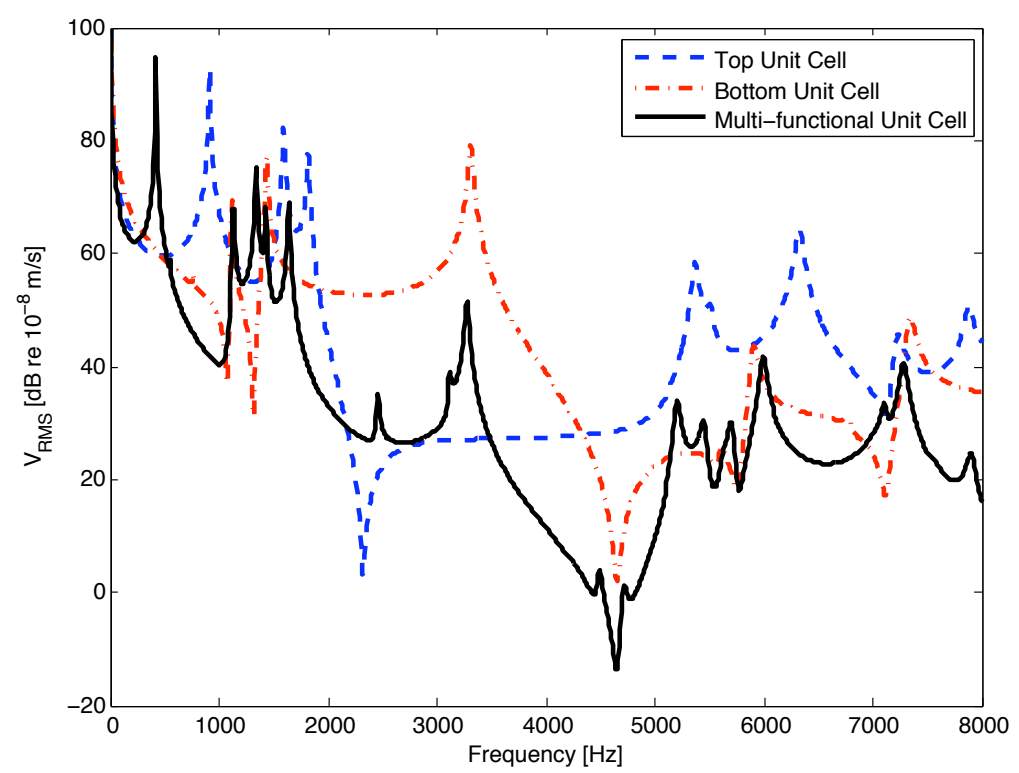

Figure 3.64: Top layer RMS velocity of bottom unit cell, top unit cell, and multifunctional unit cell

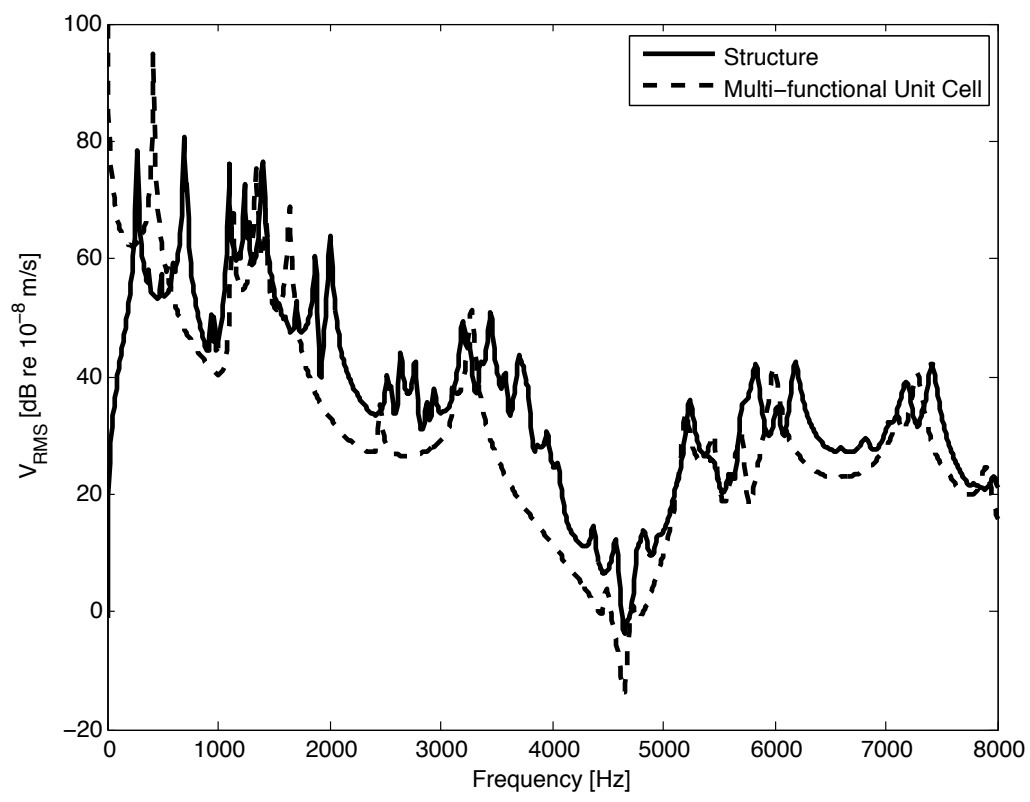

Figure 3.65: Top layer RMS velocity of multi-functional unit cell and structure 


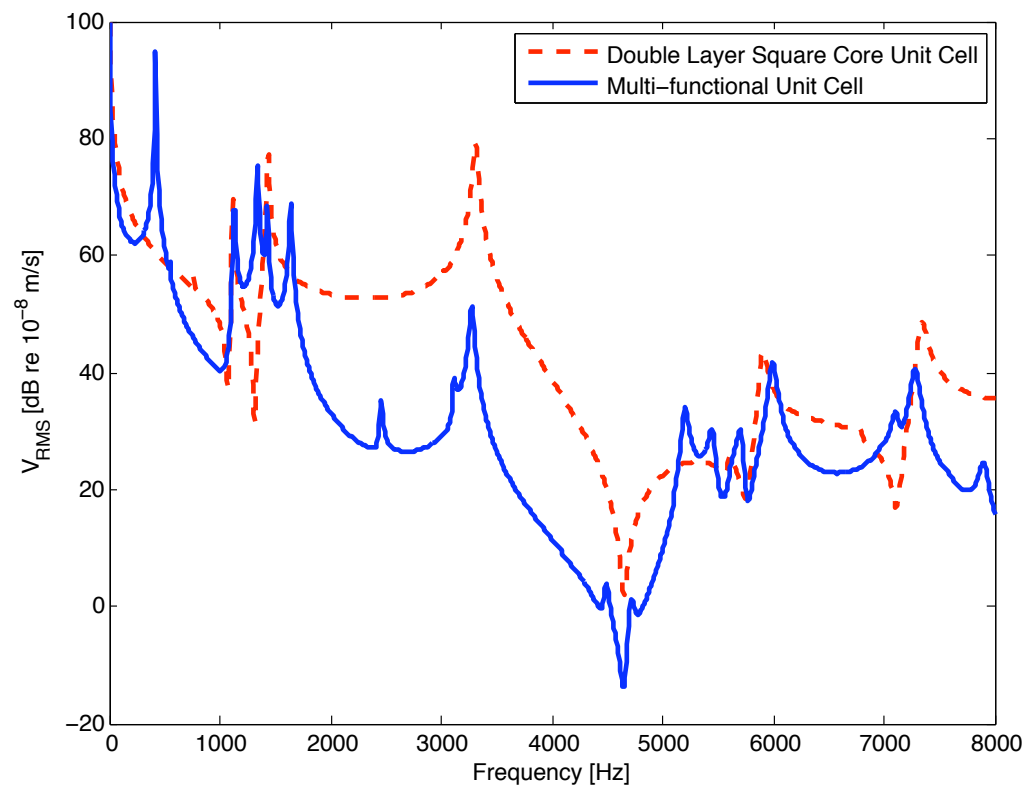

Figure 3.66: Top layer RMS velocity of double layer square core unit cell versus multi-functional unit cell

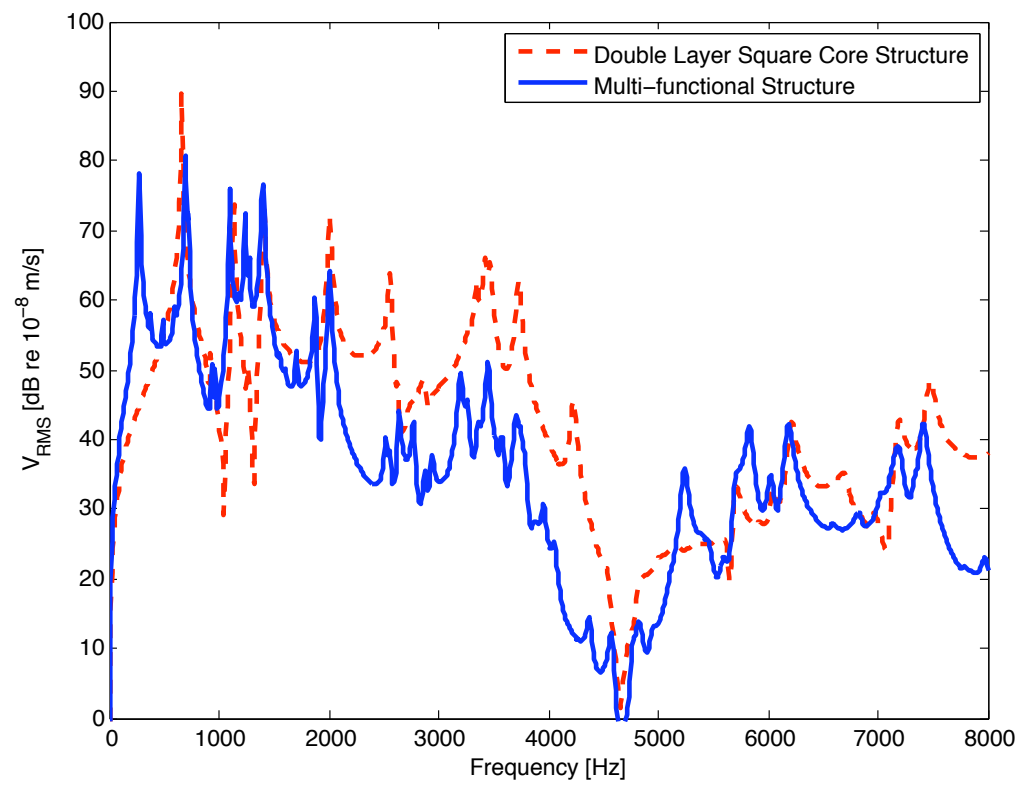

Figure 3.67: Top layer RMS velocity of double layer square core structure versus multi-functional structure 


\subsection{3-D Unit Cell \& Structure}

The final topic of this chapter is centered around the extension of the definitions, concepts, and numerical methods used in the analysis and design of 2-D truss-like structures to the study of 3-D lattice structures. The 3-D space frame spectral finite element formulation presented in Section 2.3 is utilized. Additionally, the rhombus compliant mechanism topology from Section 3.2.4 is implemented in this investigation.

\subsubsection{Concept \& Definitions}

The study of planar structures has served as an appropriate starting point for this research. Specifically, this approach has facilitated analysis while avoiding undue geometric complexity. There are, however, two shortcomings of a 2-D analysis that need to be addressed: 1) the inability of a two dimensional analysis to capture the out-of-plane component of wave propagation and 2) the limitations associated with designing practical structures in a purely planar workspace. Therefore, despite the common claim that the extension of a $2-\mathrm{D}$ method to $3-\mathrm{D}$ is trivial, it is instructive to take this step in order to enable the analysis and design of realistic, albeit more complicated, structures.

A 3-D truss unit cell and lattice panel configuration having neither a top nor a bottom facesheet is employed in this study. This configuration can be envisioned as the internal truss core contained within a composite panel. An integral rhombus compliant mechanism is oriented in both the $x$-direction and $y$-direction as illustrated by the three-dimensional unit cell model given in Figure 3.68. For simplicity, the members of each integral compliant mechanism unit cell are assumed to have a circular cross-section. 


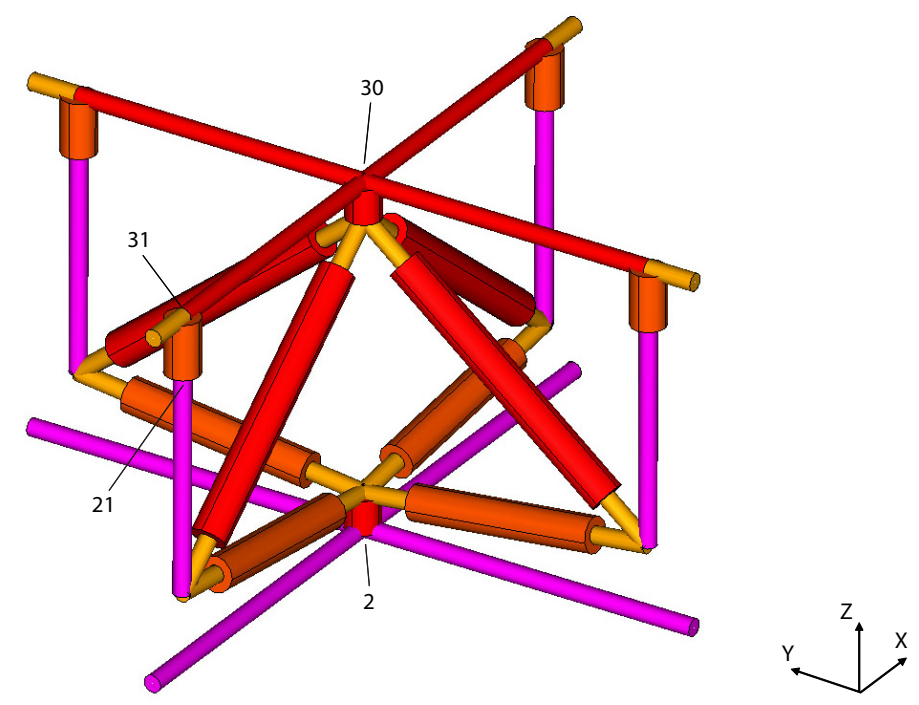

Figure 3.68: 3-D rhombus compliant mechanism unit cell model (numbers denote nodes)

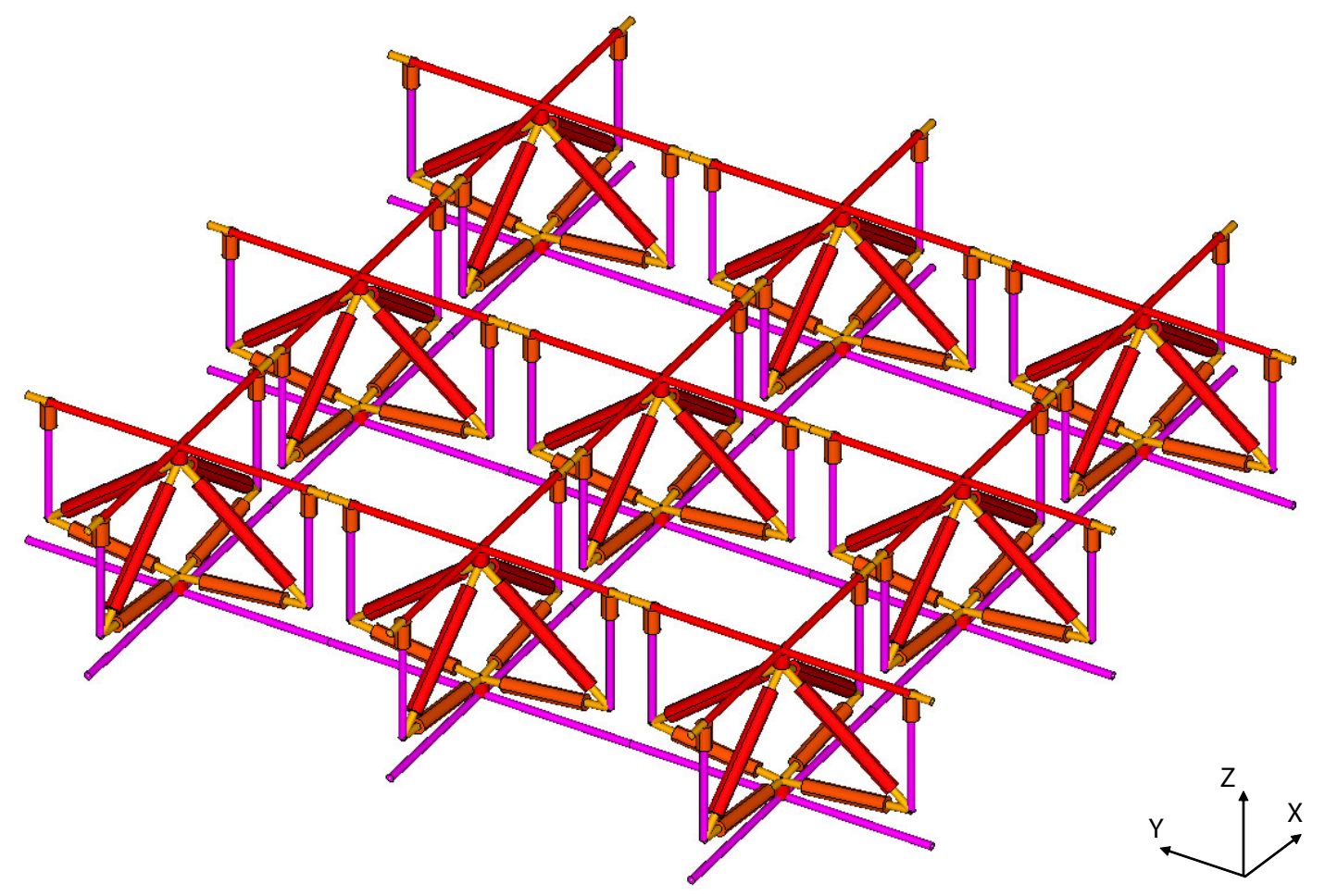

Figure 3.69: 3-D periodic rhombus compliant mechanism lattice panel structure 
The conceptual model of a periodic lattice structure is shown in Figure 3.69 consisting of nine unit cells assembled in a square panel configuration. The structure is assumed to be rigidly fixed to a baffle on all exposed sides. The bottom layer (i.e. the purple colored members that reside in the $x$ - $y$ plane) is subjected to a distributed load induced by an incident pressure wave front.

In the 3-D rhombus compliant mechanism unit cell model shown in Figure 3.68, the ground members are connected to the top layer of the lattice per the convention stipulated in Section 3.2.4. Appropriately, the FRF amplitude and phase of the 3-D unit cell model are defined as

$$
\text { Amplitude }=\left|\frac{\hat{v}_{30 z}-\hat{v}_{31 z}}{\hat{v}_{2 z}-\hat{v}_{21 z}}\right| \quad ; \quad \text { Phase }=\frac{180}{\pi}\left[\angle\left(\hat{v}_{30 z}-\hat{v}_{31 z}\right)-\angle\left(\hat{v}_{2 z}-\hat{v}_{21 z}\right)\right]
$$

where the output is taken as the $z$-direction spectral displacement of node 30 relative to node 31 , and the input is taken as the $z$-direction spectral displacement of node 2 relative to node 21 . Nodes 21 and 31 are selected since they are part of the ground of the mechanism.

Observe that in order to predict the frequency at which the attenuation of dynamic response begins, the wavenumber-frequency equation, Eqn (3.16) from Section 3.2.1, is still used.

\subsubsection{Analysis}

In Figure 3.70 the bottom layer of the structure is excited by a pressure wave front that propagates in three dimensions. This leads to the standard form for the harmonic variation of pressure in time

$$
p_{\mathrm{i}}(x, y, z, t)=p_{\mathrm{i}}(x, y, z, \omega) \mathrm{e}^{-\mathrm{i} \omega t}
$$




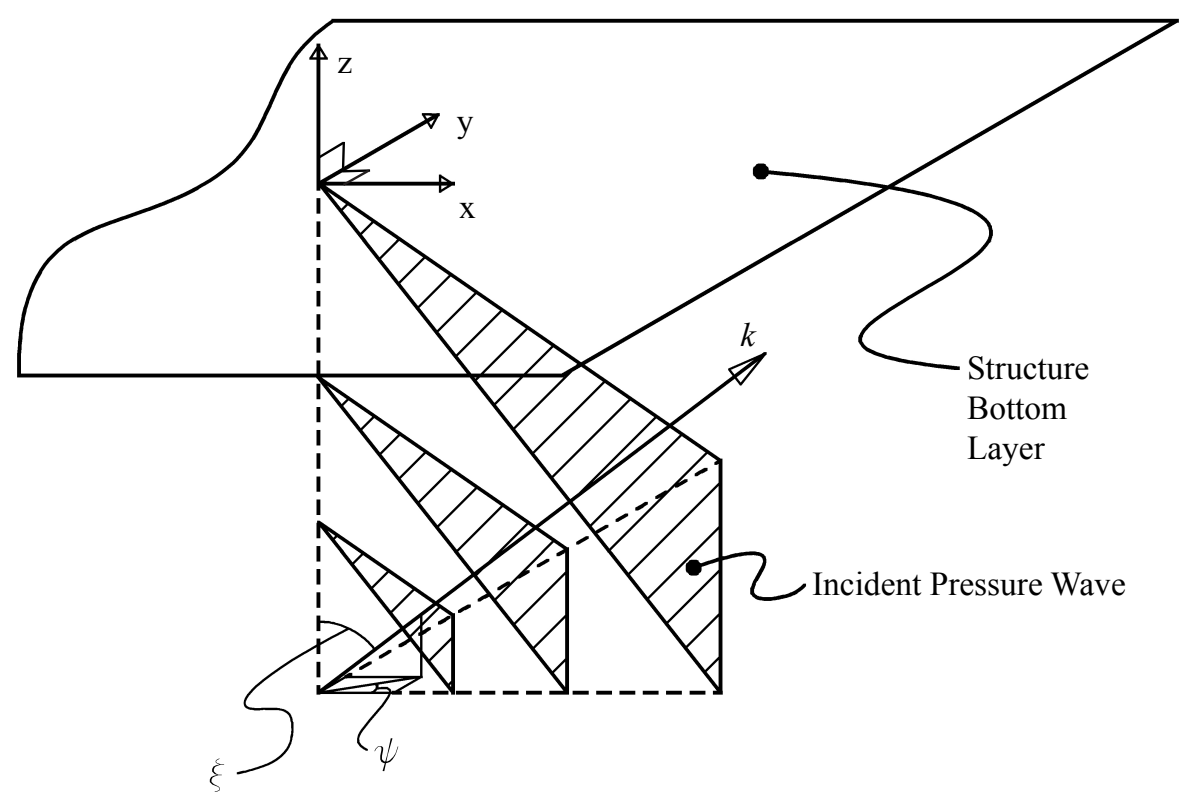

Figure 3.70: 3-D plane wave incident to the bottom layer of the structure

where

$$
p_{\mathrm{i}}(x, y, z, \omega)=p_{\text {in }} \mathrm{e}^{\mathrm{i} k_{x} x+\mathrm{i} k_{y} y+\mathrm{i} k_{z} z}
$$

In Eqn $(3.23) k_{x}, k_{y}$, and $k_{z}$ represent the cartesian components of the acoustic wavenumber, $k$, of the fluid through which the incident pressure wave travels. These components can be written in terms of the polar angle, $\xi$, and azimuthal angle, $\psi$, as

$$
\begin{gathered}
k_{x}=k \sin \xi \cos \psi \\
k_{y}=k \sin \xi \sin \psi \\
k_{z}=k \cos \xi
\end{gathered}
$$

By combining Eqns (3.24)-(3.26) with Eqn (3.23), it follows that

$$
p_{\mathrm{i}}(x, y, z, \omega)=p_{\text {in }} \mathrm{e}^{\mathrm{i} k(x \sin \xi \cos \psi+y \sin \xi \sin \psi+z \cos \xi)}
$$


The assumption of a normally incident pressure wave with $\xi=0, \psi=0$, and $z=0$ allows Eqn (3.27) to be written in a reduced form similar to Eqn (3.2) as

$$
p_{\mathrm{i}}(x, y, z, \omega)=p_{\text {in }}
$$

Thus, given this formulation of the pressure, the necessary components of the equivalent spectral load vector for a space frame element residing in the bottom layer $x-y$ plane are found to have the same form given previously in Eqns (3.7) and (3.8).

\subsubsection{Computational Results}

The three-dimesional unit cell and structure are assumed to be made of aluminum. An additional material physical parameter is required for a three dimensional analysis. The Poisson's ratio, $\nu=0.33$, allows for the calculation of the material complex shear modulus, $G^{*}$, using the relation $G^{*}=E^{*} /(2(1+\nu))$.

The boundary conditions for the unit cell analysis are the 3-D counterparts to the symmetry boundary conditions used in all of the 2-D unit cell analyses. Rotational degrees of freedom are constrained for the nodes that lie on a vertical boundary of the unit cell in either a $y-z$ plane or $z-x$ plane, referring to Figure 3.68. For the full panel structure, all six degrees of freedom (i.e. both rotations and translations) of nodes that lie on a vertical boundary of the structure in either a $y$ - $z$ plane or $z$-x plane are constrained, (see Figure 3.69).

The unit cell dimensions are $0.1 \mathrm{~m}$ in the $x$-direction by $0.1 \mathrm{~m}$ in the $y$-direction by $0.05 \mathrm{~m}$ in the $z$-direction. The lattice panel structure has dimensions of $0.3 \mathrm{~m}$ in both the $x$ and $y$ directions and a thickness of $0.05 \mathrm{~m}$ in the $z$-direction. The diameter of the bottom layer members of the unit cell that reside in the $x-y$ plane is $2.5 \mathrm{~mm}$. The core members of the unit cell are assigned a diameter of $3.75 \mathrm{~mm}$. All remaining members (i.e. ground, transmission, etc.) are assigned a diameter of 5.0 


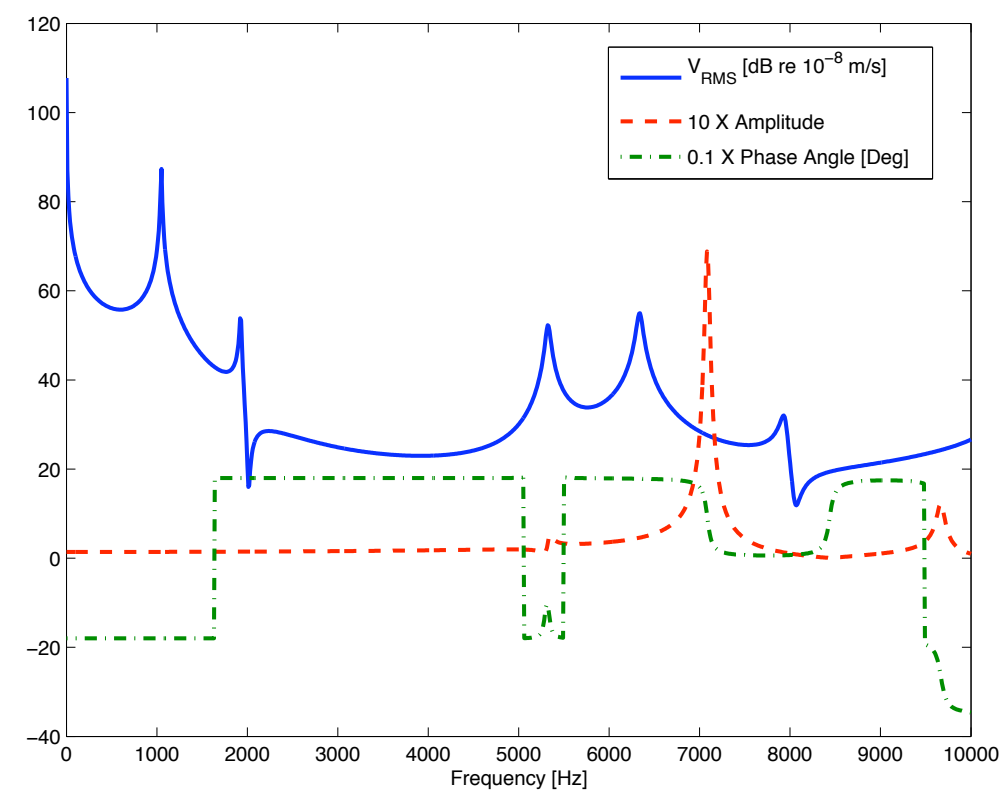

Figure 3.71: 3-D rhombus compliant mechanism unit cell FRF

$\mathrm{mm}$.

The frequency range of interest for this study is $0 \mathrm{~Hz}$ to $10 \mathrm{kHz}$, with response computed at $10 \mathrm{~Hz}$ frequency intervals. The 3-D unit cell FRF is given in Figure 3.71. Below $2 \mathrm{kHz}$ a single prominent peak in RMS $z$-direction velocity response is visible at $1050 \mathrm{~Hz}$. This peak represents a resonance of the unit cell prior to the attenuation zone starting frequency. Substitution of the physical parameters of the bottom layer elements into Eqn (3.16), along with $\lambda=0.1 \mathrm{~m}$ (i.e. the length of the unit cell in both the $x$ and $y$ directions), gives the first attenuation starting frequency as 2030 Hz. Observe that the selection of a circular cross-section affects the attenuation zone starting frequency by lowering it slightly relative to that of the two-dimensional rhombus compliant mechanism unit cell. However, similar to the 2-D unit cell, the FRF illustrates that within the attenuation zone the 3-D rhombus compliant mechanism exhibits non-resonant (i.e. small amplitude), out-of-phase "inversor" 


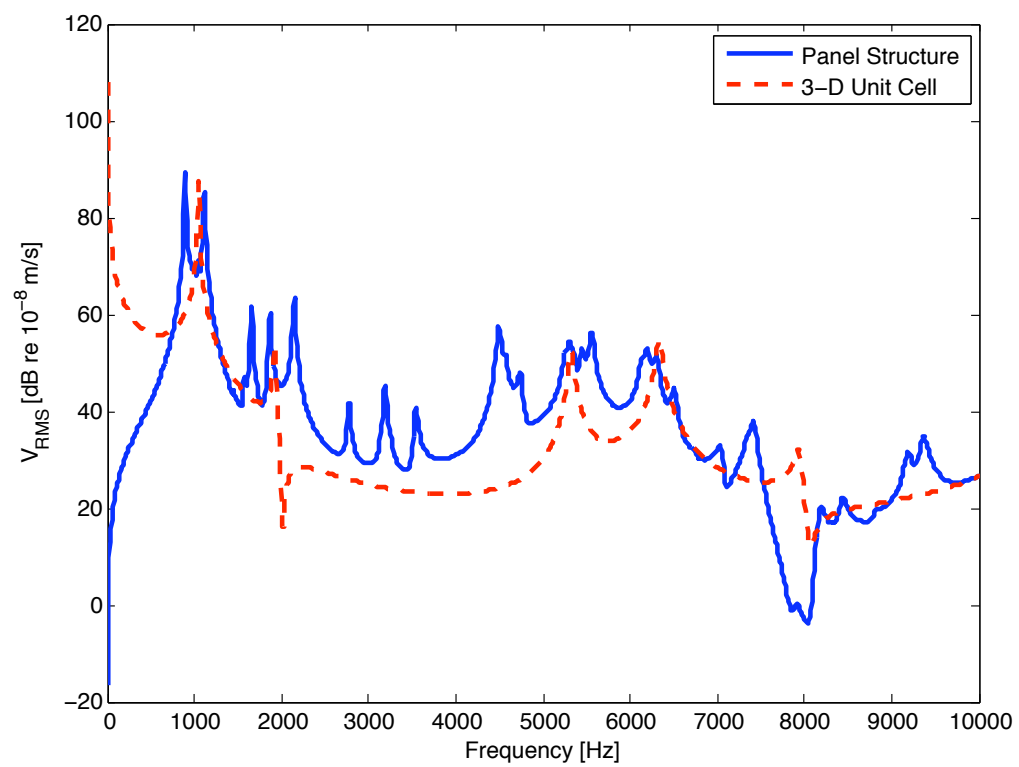

Figure 3.72: Top layer RMS velocity of 3-D lattice panel structure versus unit cell

behavior. Beyond $5 \mathrm{kHz}$ the two RMS $z$-direction velocity response peaks at 5310 $\mathrm{Hz}$ and $6350 \mathrm{~Hz}$ represent resonances of the compliant mechanism core. In the case of the first resonant peak at $5310 \mathrm{~Hz}$, the increase in velocity amplitude is associated with a slight increase in FRF amplitude and a FRF phase that briefly moves closer to an in-phase angle. At $8 \mathrm{kHz}$ the second attenuation zone is present as predicted by Eqn (3.16) when $\lambda=0.05 \mathrm{~m}$ (i.e. the half-length of the unit cell in both the $x$ and $y$ directions). Moving towards $10 \mathrm{kHz}$ the RMS $z$-direction velocity response again begins to rise in magnitude as FRF amplitude increases.

The 3-D lattice panel structure RMS $z$-direction velocity response is compared with the response of the 3-D unit cell in Figure 3.72. The first resonance of the panel structure occurs at $890 \mathrm{~Hz}$ and carries over into a second peak in close proximity to $1120 \mathrm{~Hz}$. Just prior to the first attenuation zone, the second unit cell resonant peak near $2 \mathrm{kHz}$ is split into three peaks in the response of the panel structure. Lower 
amplitude resonances are also visible within the first attenuation zone. Specifically, three closely spaced peaks are noticeable at $2780 \mathrm{~Hz}, 3190 \mathrm{~Hz}$, and $3540 \mathrm{~Hz}$, along with a broad peak from 4400-4700 Hz. However, the first attenuation zone is still present and is only slightly limited by the occurrence of these resonances. Beyond $5 \mathrm{kHz}$ the RMS z-direction velocity response of the panel structure matches, in an approximate sense, the response of the unit cell. The second attenuation zone starts at a somewhat lower frequency of $7800 \mathrm{~Hz}$. Increased modal density within the second attenuation zone is evident near $9400 \mathrm{~Hz}$.

The total energy in each 3-D spectral space frame element of the unit cell and structure was computed at specific frequencies using Eqn (3.18). The deformed shape of the unit cell and total energy distribution at $2500 \mathrm{~Hz}$, within the first attenuation zone, is shown in Figure 3.73 (a). Thicker lines denote greater energy content, and energy isolation in the bottom layer of the unit cell is visible. The first mechanism resonance at $5310 \mathrm{~Hz}$ is confirmed through examination of Figure 3.73 (b); a greater transference of energy into the core of the unit cell occurs. The total energy distribution at $9000 \mathrm{~Hz}$, within the second attenuation zone, is shown in Figure $3.73(\mathrm{c})$. The energy isolation phenomenon is again remarkable.

From a global perspective, examining the total energy distribution within the lattice panel structure, exclusive of the deformed shape, elucidates the underlying mechanisms behind increased modal density within the first attenuation zone. The total energy distribution within the panel structure is viewed edge on for clarity at numerous frequencies as shown in Figure 3.74. Specifically, results are shown at frequencies just before, at, and in between the resonances at $2780 \mathrm{~Hz}, 3190 \mathrm{~Hz}, 3540$ $\mathrm{Hz}$, and the broad resonance centered around $4490 \mathrm{~Hz}$. Again, thicker lines denote greater energy content. 


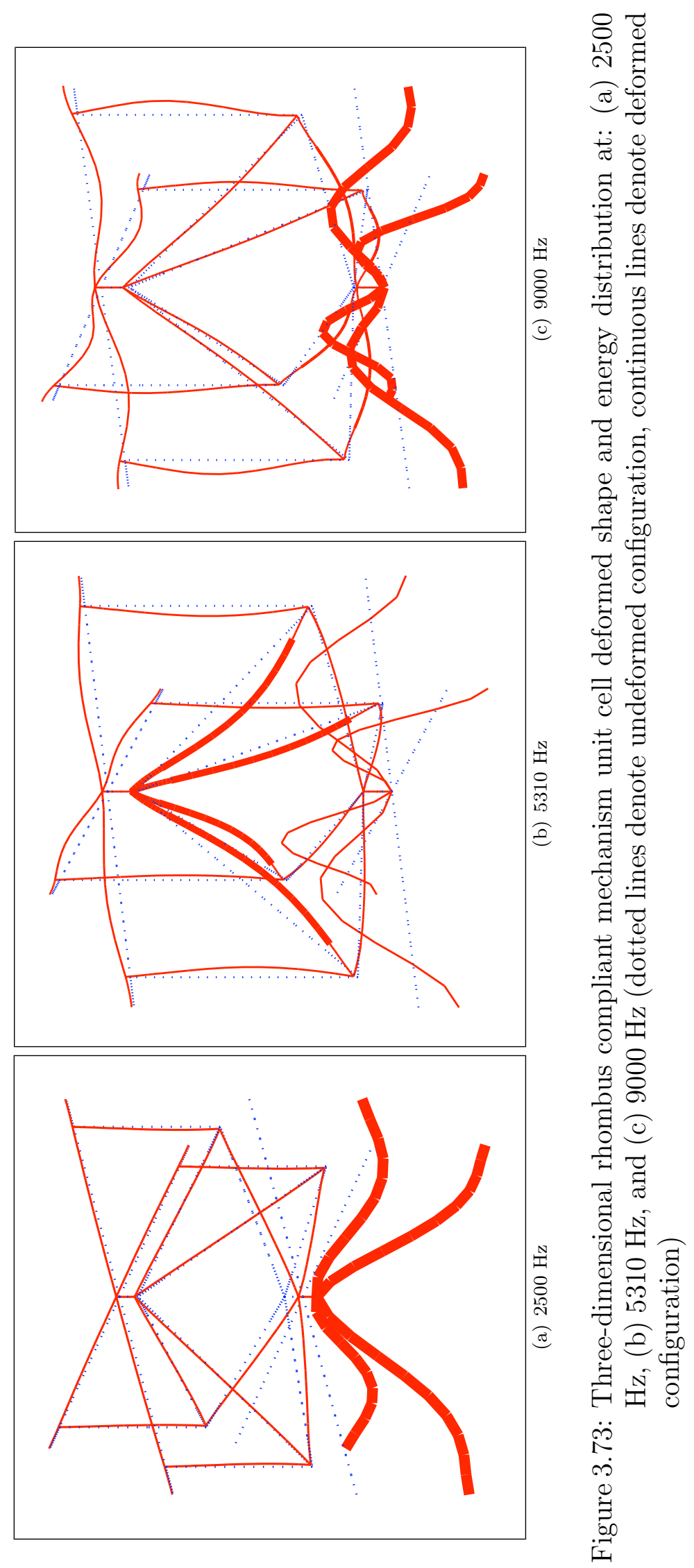




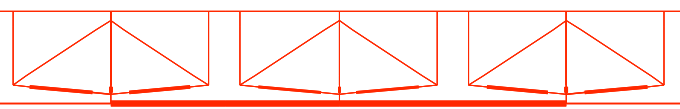

(a) $2650 \mathrm{~Hz}$ (just prior to first resonance)

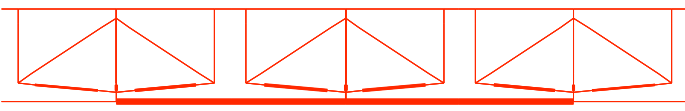

(c) $3000 \mathrm{~Hz}$ (between first and second resonances)

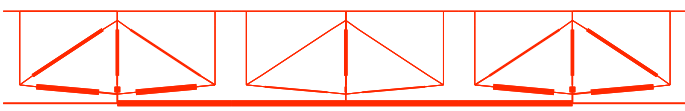

(e) $3430 \mathrm{~Hz}$ (between second and third resonances)

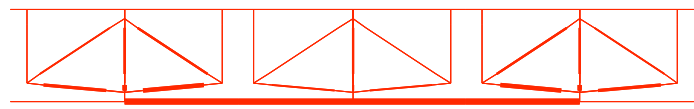

(g) $3840 \mathrm{~Hz}$ (just after third resonance)

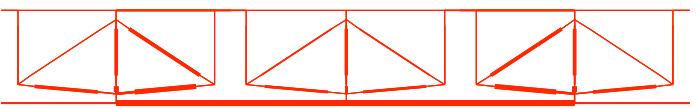

(b) $2780 \mathrm{~Hz}$ (first resonance)

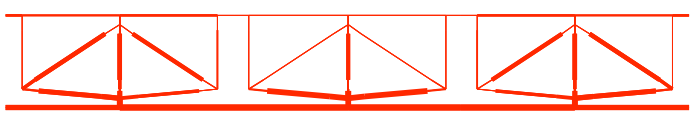

(d) $3190 \mathrm{~Hz}$ (second resonance)

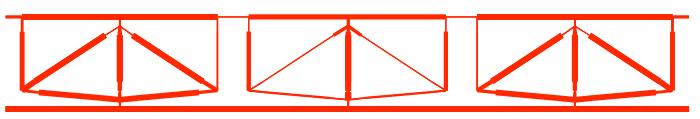

(f) $3540 \mathrm{~Hz}$ (third resonance)

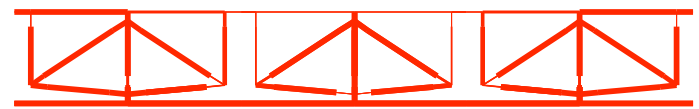

(h) $4490 \mathrm{~Hz}$ (fourth resonance)

Figure 3.74: 3-D rhombus compliant mechanism lattice panel structure energy distribution - viewed edge on for visual clarity - at the following frequencies: (a) $2650 \mathrm{~Hz}$, (b) $2780 \mathrm{~Hz}$, (c) $3000 \mathrm{~Hz}$, (d) $3190 \mathrm{~Hz}$, (e) $3430 \mathrm{~Hz}$, (f) $3540 \mathrm{~Hz}$, (g) $3840 \mathrm{~Hz}$, and (h) $4490 \mathrm{~Hz}$

At frequencies before or after a resonance, Figures 3.74 (a), (c), (e), and (g), energy is generally confined to the lower half of the structure. In contrast to this behavior, at each resonance there is a localization of energy within specific portions of the panel structure, Figures 3.74 (b), (d), (f), and (h), plus energy transfer throughout the structure including the panel core, and more significantly, the panel top layer. These local resonances are not captured by the standalone unit cell analysis since they are related to the interaction between different unit cells once assembled into a global structure. This is also supported by the observation that, for the global structure, the number of resonant peaks within regions of increased modal density tends to be correlated with the periodicity of the structure. In this specific example, the lattice panel has dimensions that are defined by three unit cells oriented in both the $x$ and $y$ directions, and in the regions of increased modal density resonant peaks 
tend to appear in groups of three. This global interaction of local unit cells makes clear the limitations associated with unit cell reduced order computational models.

Prior to leaving this chapter, a final picture of the global dynamic behavior of the 3-D lattice panel structure is given by computing the total energy distribution throughout the structure at two frequencies. Isometric views at $2650 \mathrm{~Hz}$ and 8040 $\mathrm{Hz}$ are provided in Figures 3.75 (a) and (b), respectively. Top layer energy isolation is prevalent as visualized through a thinner line thickness. 


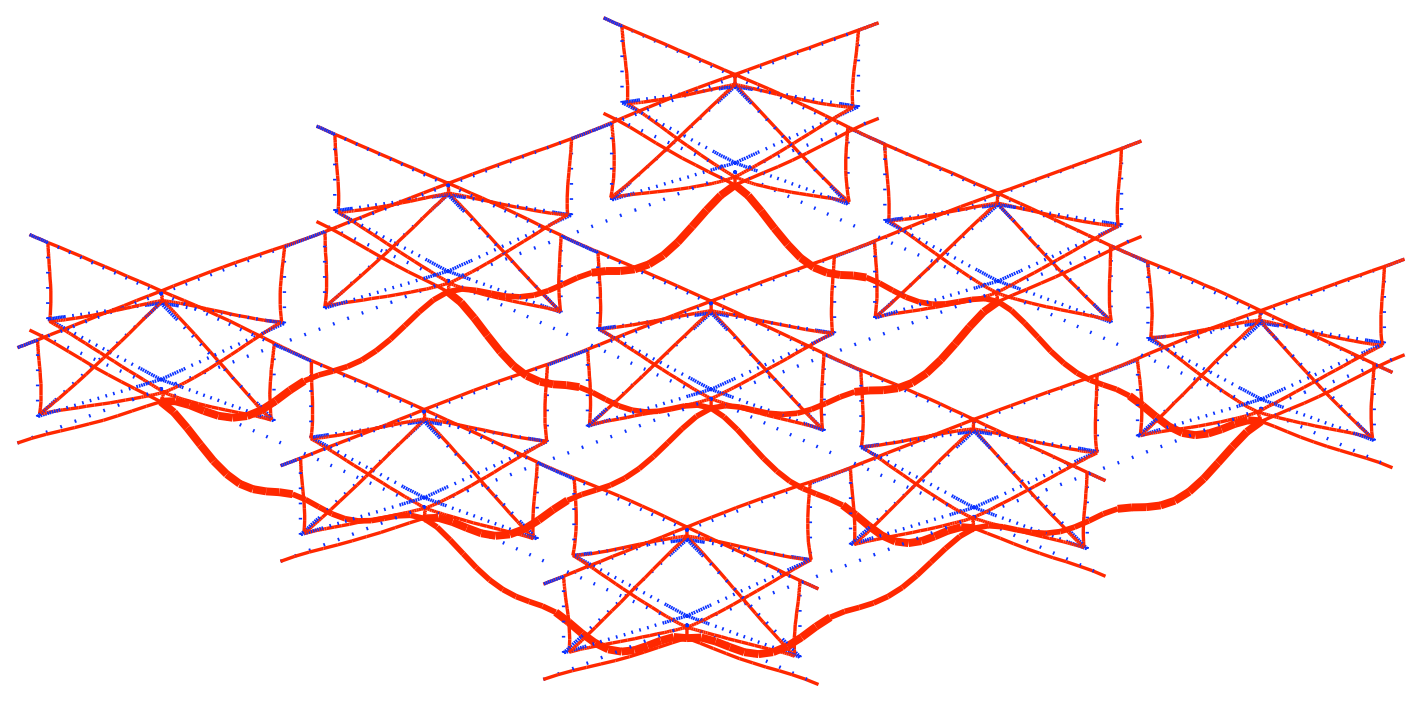

(a) $2650 \mathrm{~Hz}$

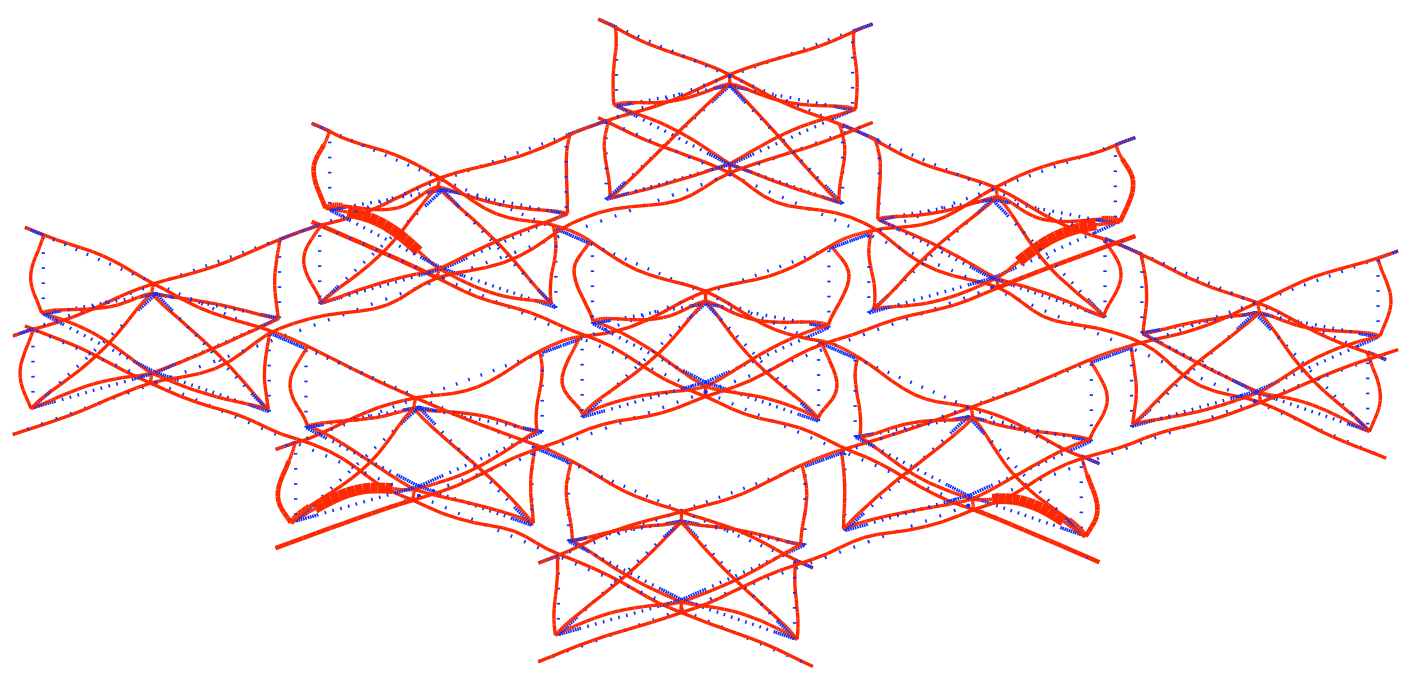

(b) $8040 \mathrm{~Hz}$

Figure 3.75: 3-D rhombus compliant mechanism lattice panel structure deformed shape and energy distribution at the following frequencies: (a) 2650 $\mathrm{Hz}$ and (b) $8040 \mathrm{~Hz}$ (dotted lines denote undeformed configuration, continuous lines denote deformed configuration) 


\section{CHAPTER 4}

\section{Genetic Algorithm Optimization}

In the previous chapters, numerous computational models and results have been presented. These studies have focused on establishing several ideas fundamental to this research including the compliant mechanism unit cell analysis and design concept, the design of unit cells composed of alternative materials, the connection of unit cells in parallel and series fashion, and the extension of the analysis and design methodology to three dimensions. The next step in formulating a unified methodology is to investigate the optimization of structures with integral compliant mechanisms in order to achieve a desired response over a prescribed frequency range. In this chapter a genetic algorithm optimization routine is coupled to the aforementioned spectral finite element computational analysis.

In Section 4.1, size optimization of structural members within a compliant mechanism unit cell is performed using a genetic algorithm. Planar structures are once more the subject of investigation, and the objective is to "tune" the response of an assumed topology. This topic was discussed briefly in various parts of Section 3.4, however, the size of unit cell members was adjusted manually to achieve the desired structural dynamic response. In this section the size optimization procedure is automated.

In Section 4.2, a genetic algorithm is further employed for the topology optimization of the unit cell. A starting network structure is assumed, from which final unit 
cell topologies are computed. The goal in this section is to introduce topology optimization as another method for deriving compliant mechanism unit cell designs that exhibit enhanced structural dynamic performance.

\subsection{Size Optimization}

The genetic algorithm used for size optimization has the standard operations, per Goldberg [30], found in most applications: 1) selection of candidate individuals from an initial population according to their fitness value; 2) crossover, where portions of two individuals are combined to create a new individual; 3) mutation, where small, random changes are introduced. Additionally, an elitist survival strategy is implemented to ensure that the fittest individual from a given population survives in the next generation, as discussed by Keane [50].

\subsubsection{Analysis}

It is appropriate to define an objective in terms of "response tuning." As mentioned in Section 3.2.1, changing the thickness of various beam widths within the unit cell alters the attenuation zone breadth (due to modification of the mechanism stiffness and consequently natural frequencies). Hence, the objective for this study is to use a genetic algorithm to optimize the size of various beams in the unit cell and decrease the RMS normal velocity response over a specified frequency range. Specifically, the objective function is to minimize the following fitness value (FV)

$$
\min \mathrm{FV}=\sum_{n} \mathrm{v}_{\mathrm{RMS}}
$$

where the RMS velocity, $\mathrm{v}_{\mathrm{RMS}}$, is summed over the, $n$, number of frequencies spanning a frequency interval.

The compliant mechanism unit cell from Section 3.2.1 is used as a template for 


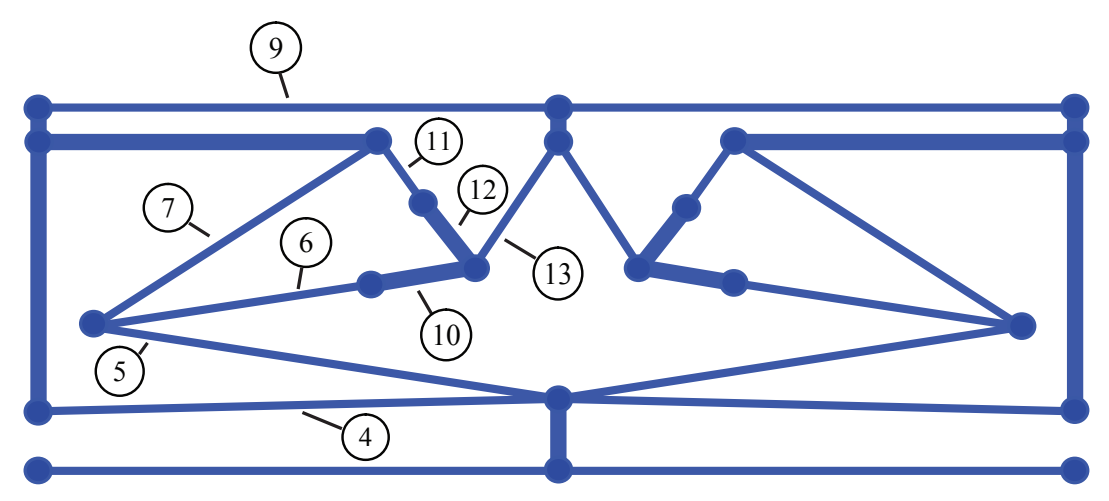

Figure 4.1: Compliant mechanism unit cell model (numbers that are circled denote elements)

this study. For the size optimization analysis a total of nine element widths are used as variables; one for each of the following elements: 4 through 7 and 9 through 13 (Figure 4.1). Symmetry is enforced about the centerline of the unit cell. The elements chosen for size optimization include the mechanism core and top layer elements, but not the ground, transmission, or bottom layer elements. The latter groups, not selected for size optimization, are considered paramount to realizing response attenuation. For example, the bottom layer element width should not be modified since that will change the frequency at which the attenuation zone begins, according to Eqn (3.16).

A flow chart of computations for the unit cell genetic algorithm size optimization procedure is given in Figure 4.2. The analysis procedure requires the generation of an initial random population after which spectral finite element analyses are conducted to compute the fitness value for each individual over the specified frequency range. Following this, the optimizer performs selection, crossover, and mutation, and the fitness values of the new population members are computed using the spectral finite element analysis. This procedure is iterated over a set number of generations and 


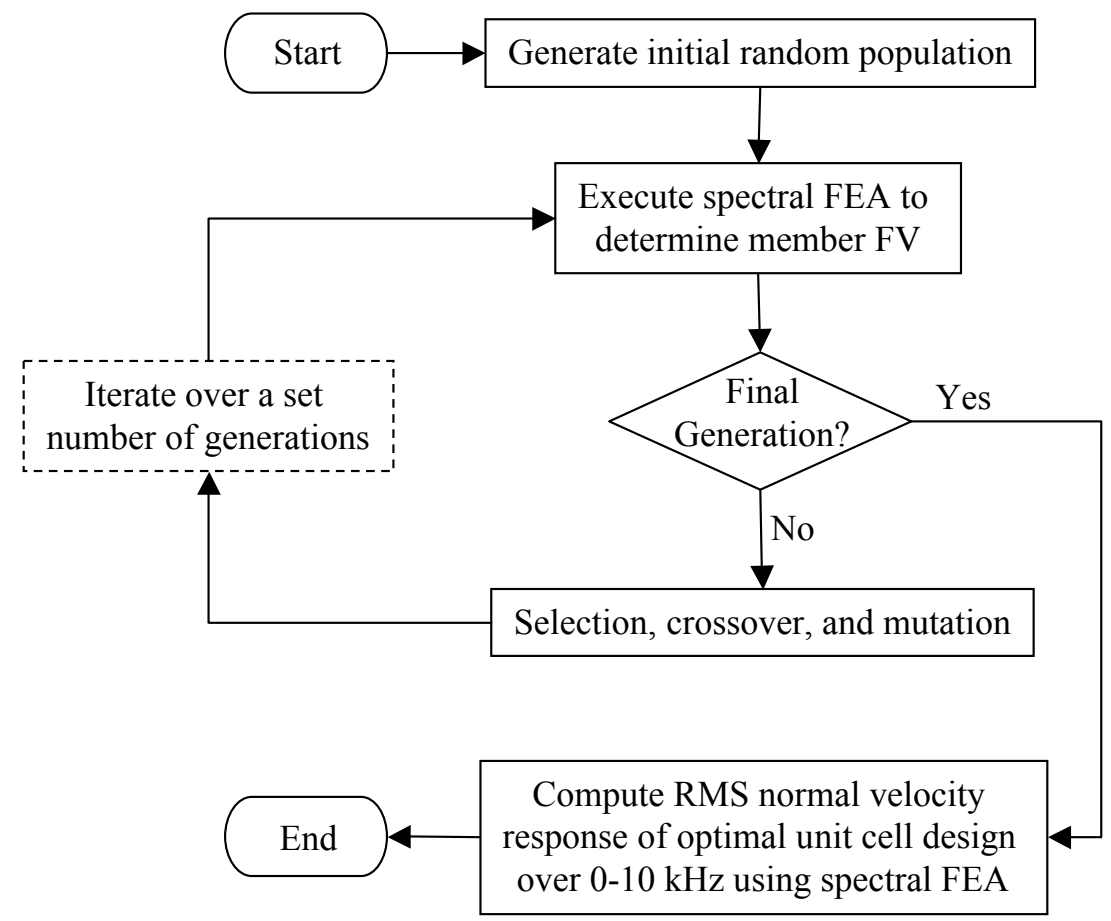

Figure 4.2: Flowchart of computations for genetic algorithm size optimization

the final size optimized design is obtained.

\subsubsection{Results}

The width of each element was allowed to vary $+75 \% /-50 \%$ from an initial value of $2.5 \mathrm{~mm}$. An 18-bit binary encoding was used in the genetic algorithm for the nine variables (i.e. 2 bits per variable). A population size of 10 was iterated over four generations. Given the range of fitness values for the members of the population, individuals within the top $80 \%$ were selected and copied into a mating pool with fitter members more represented. Among those surviving individuals, $80 \%$ of them were allowed to crossover (i.e. breed) after which, a mutation rate of $1 \%$ was applied.

The compliant mechanism unit cell was optimized to minimize the FV over the frequency interval of $5-7 \mathrm{kHz}$ at frequency steps of $10 \mathrm{~Hz}$ with $n=200$ in Eqn (4.1). In particular, initial response peaks at $5400 \mathrm{~Hz}$ and $6200 \mathrm{~Hz}$, as shown in Figure 4.3, 


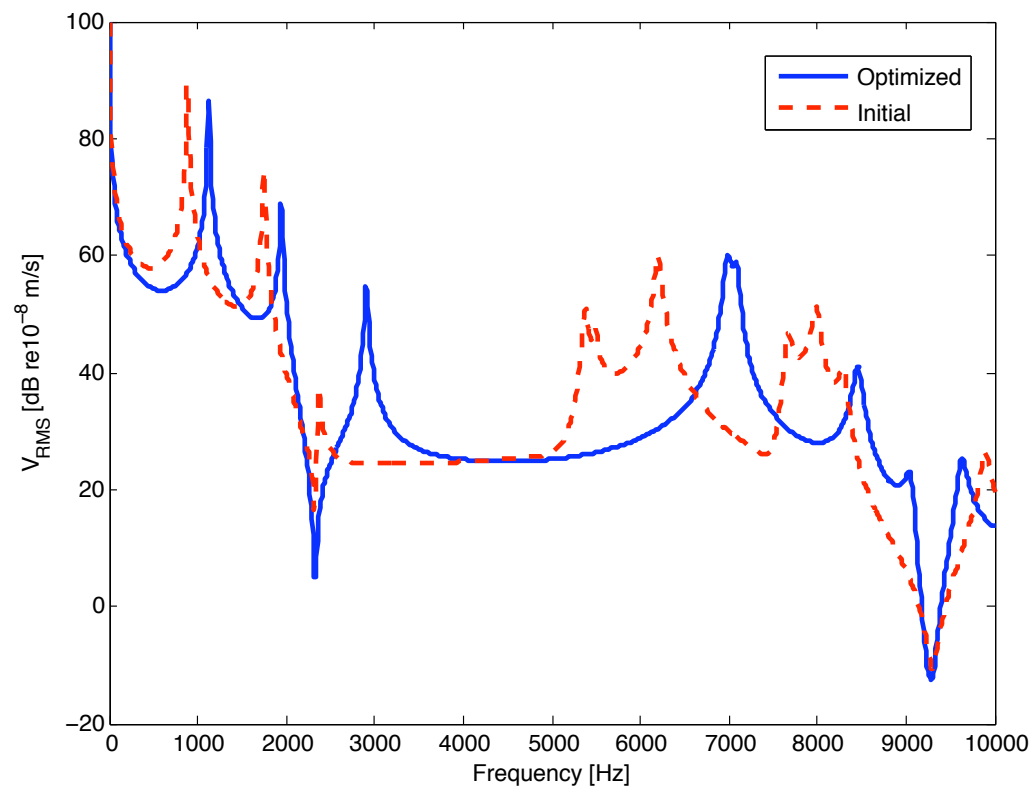

Figure 4.3: Top layer RMS velocity of compliant mechanism unit cell

were targeted for reduction. This figure also shows the RMS transverse velocity of the optimized unit cell response computed over the entire $0 \mathrm{~Hz}$ to $10 \mathrm{kHz}$ range at $10 \mathrm{~Hz}$ frequency steps. An average reduction of $9 \mathrm{~dB}$ was achieved over the $5 \mathrm{kHz}$ to $7 \mathrm{kHz}$ frequency range with a significant reduction of $25 \mathrm{~dB}$ at $5400 \mathrm{~Hz}$ and 30 $\mathrm{dB}$ at $6200 \mathrm{~Hz}$. Comparing the initial and optimized responses, it is clear that the unit cell resonances have been shifted to the right across the majority of the $10 \mathrm{kHz}$ range implying a somewhat stiffer structure.

The final width of each size optimized element is given in Table 4.1 and the initial and optimized unit cell structures are shown in Figures 4.4 and 4.5, respectively. Element width is represented by varying line thickness in these figures. The thicker members in the optimized structure are consistent with the notion of the unit cell being stiffer, leading to a shift of the unit cell resonances towards higher frequencies. These optimization results are excellent given that only four generations were 
Table 4.1: Initial and optimized sizing of the compliant mechanism unit cell

\begin{tabular}{ccc}
\hline Element Number & Initial Width & Optimized Width \\
\hline 4 & $2.5 \mathrm{~mm}$ & $3.3 \mathrm{~mm}$ \\
5 & $2.5 \mathrm{~mm}$ & $3.3 \mathrm{~mm}$ \\
6 & $2.5 \mathrm{~mm}$ & $3.3 \mathrm{~mm}$ \\
7 & $2.5 \mathrm{~mm}$ & $4.3 \mathrm{~mm}$ \\
9 & $2.5 \mathrm{~mm}$ & $4.3 \mathrm{~mm}$ \\
10 & $2.5 \mathrm{~mm}$ & $2.3 \mathrm{~mm}$ \\
11 & $2.5 \mathrm{~mm}$ & $3.3 \mathrm{~mm}$ \\
12 & $2.5 \mathrm{~mm}$ & $4.3 \mathrm{~mm}$ \\
13 & $2.5 \mathrm{~mm}$ & $4.3 \mathrm{~mm}$
\end{tabular}

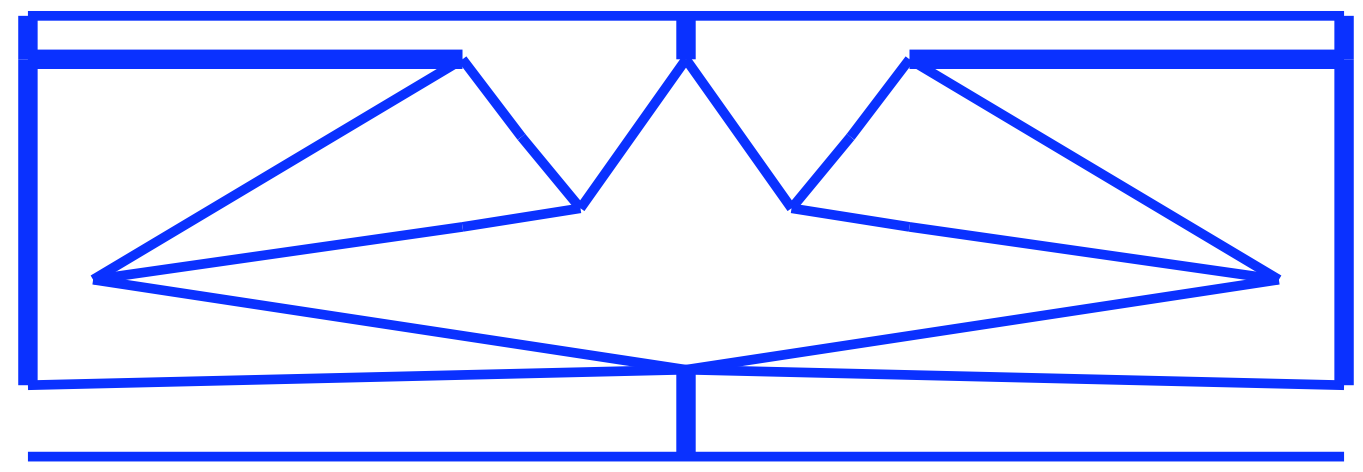

Figure 4.4: Initial compliant mechanism unit cell

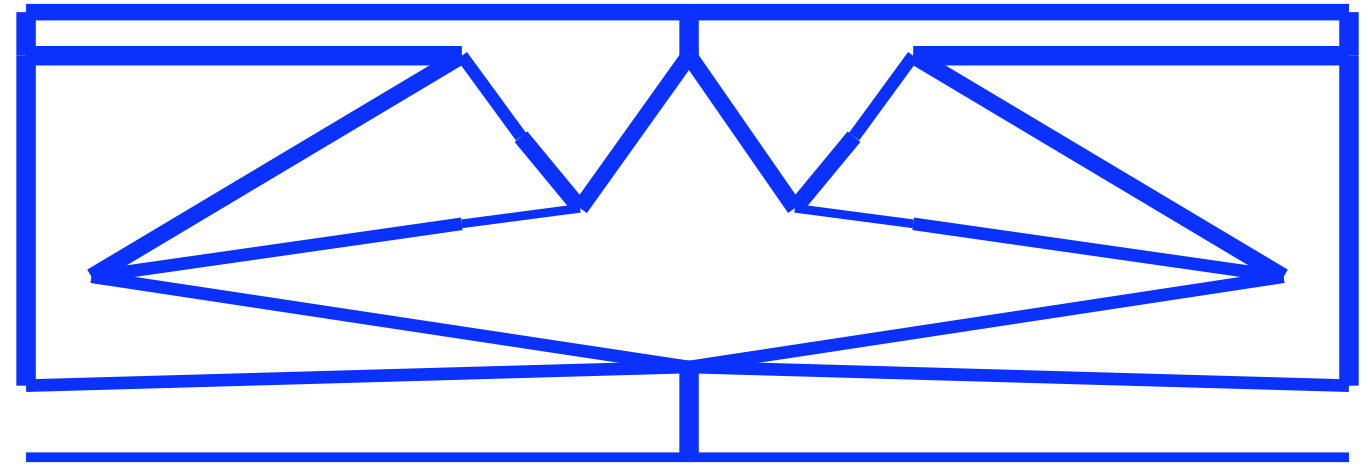

Figure 4.5: Optimized compliant mechanism unit cell 
evaluated, each with a population size of only 10 individuals.

\subsection{Topology Optimization}

In the previous section a unit cell topology was considered based on a priori knowledge of desired compliant mechanism characteristics (e.g. large mechanical advantage). In this section the unit cell topology, or geometric form, is derived using a genetic algorithm topology optimization routine. The goal here is to illuminate other possible unit cell structures that have potential in realizing a preferred midfrequency dynamic response.

\subsubsection{Analysis}

A simple unit cell binary ground structure consisting of a truss network of interconnected frame elements is established to represent the seed for the topology optimization problem. In Figure 4.6 the bottom layer of the unit cell is connected via a single transmission element to the ground structure optimization domain. The ground structure optimization domain is then connected via multiple connections to the unit cell top layer. This configuration is assumed based on the knowledge that transverse waves propagating through the unit cell bottom layer will actuate a compliant mechanism residing within the core of the unit cell. For the purposes of this study, frame elements within the 2-D optimization domain are allowed to cross over

each other. While this configuration is not physically possible in the construction of a prototype, the approach satisfies the primary goal of this investigation, which is to demonstrate the potential of the topology optimization method.

In computing an optimum topology, frame elements within the truss network are allowed to vary in thickness over a preset width range. The topology optimization problem then becomes a size optimization problem that proceeds as provided in 


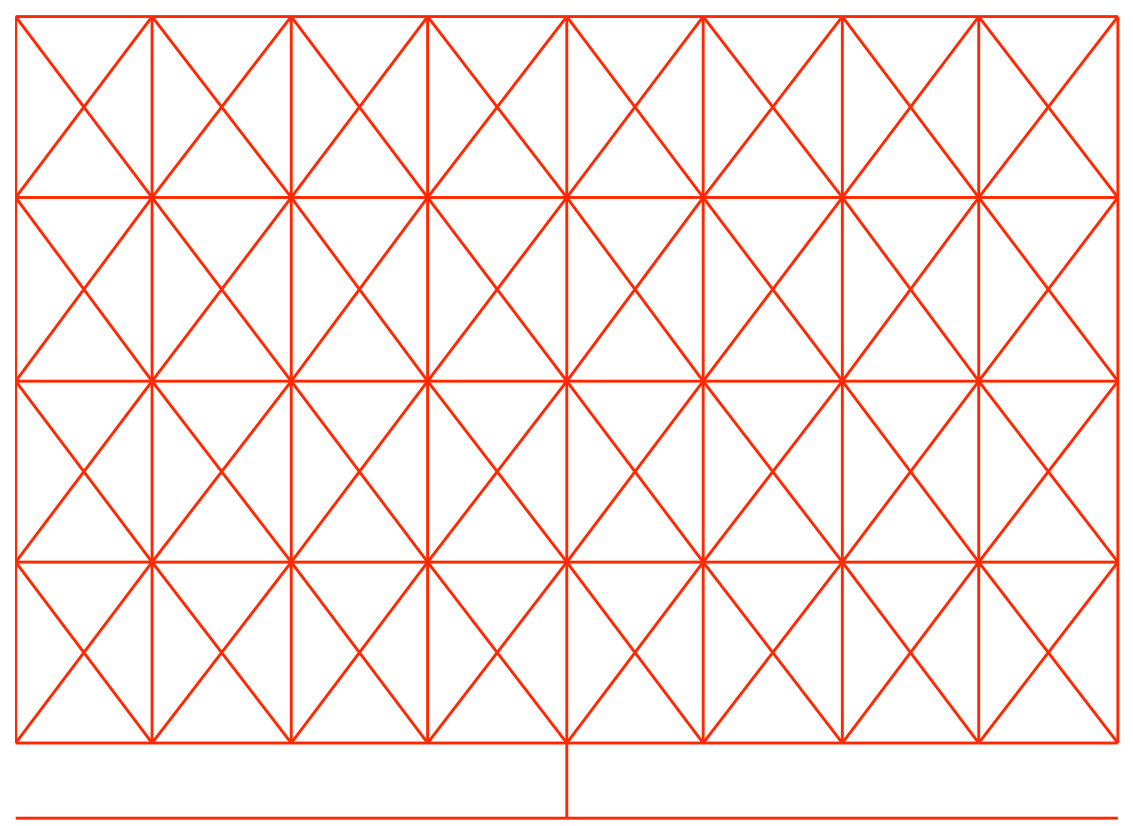

Figure 4.6: Initial (pre-optimization) unit cell binary ground structure

flowchart form in Figure 4.7. First, an initial random population of members (i.e. unit cells) is generated. Each member of this population has a randomly assigned distribution of element thicknesses for those elements that lay in the optimization domain. Second, the top layer RMS transverse velocity response of each unit cell is computed over a reduced frequency range using the spectral finite element analysis method. Following this, the fitness value of each unit cell is computed over the reduced frequency range using Eqn (4.1). The optimizer then performs selection, crossover, and mutation, and the fitness values of the new population members are determined in a similar manner via the spectral finite element analysis. This procedure is repeated over a predetermined number of iteration generations to arrive at a final generation. Third, a reduced subset of the final generation is selected, and the top layer RMS transverse velocity response of each "initial unit cell" within this 


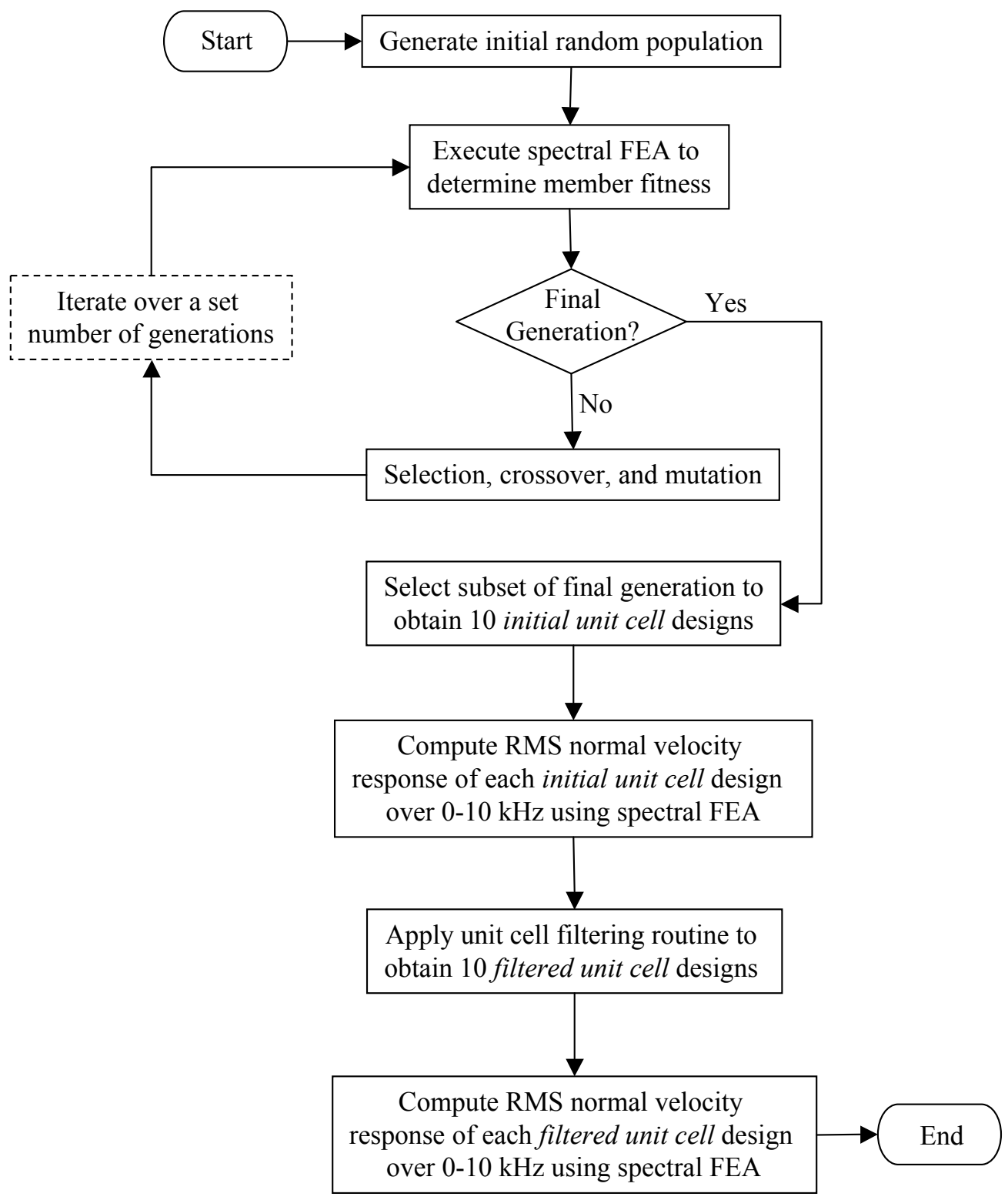

Figure 4.7: Flowchart of computations for genetic algorithm topology optimization 
subset is computed over the entire $10 \mathrm{kHz}$ mid-frequency range of interest. Fourth, a filtering routine is applied to each member of the subset to arrive at a group of final unit cell topologies. The filtering routine sets the width of elements below a predetermined threshold to a number very close to zero. Elements with small widths contribute negligibly to the overall unit cell dynamic response characteristic. This filtering approach is utilized as a way of automating the filtering process and facilitating computational efficiency since it avoids the requirement of having to regenerate the finite element mesh. Lastly, the top layer RMS transverse velocity response of each "filtered unit cell" is once more computed over the $10 \mathrm{kHz}$ frequency range and compared with the respective initial unit cell response. In such a way, final unit cell topologies that have reduced internal geometric complexity are obtained.

\subsubsection{Results: Example 1}

The unit cell seed for all of the remaining examples in this chapter is assumed to be made of aluminum and has overall dimensions of $0.1 \mathrm{~m}$ by $0.055 \mathrm{~m}$. The starting width of the core elements of the unit cell is $0.5 \mathrm{~mm}$. These core elements are allowed to range in thickness from $0.005 \mathrm{~mm}$ to $0.995 \mathrm{~mm}$ during the optimization routine. The top and bottom layer thickness is held fixed at $2.5 \mathrm{~mm}$, and the predefined ground elements of the unit cell are assigned a non-varying width of $5.0 \mathrm{~mm}$. Figure 4.8 shows the initial sizing of the unit cell where the thickest lines represent the ground elements; the thinnest lines represent the core elements that define the optimization domain; the lines that have an intermediate thickness represent elements that are part of the top and bottom layers of the unit cell.

A 670-bit binary encoding was used in the genetic algorithm optimization routine for the 67 core element width variables (i.e. 10 bits per variable). Several optimization runs were performed. In this first example, a population size of 140 members was 


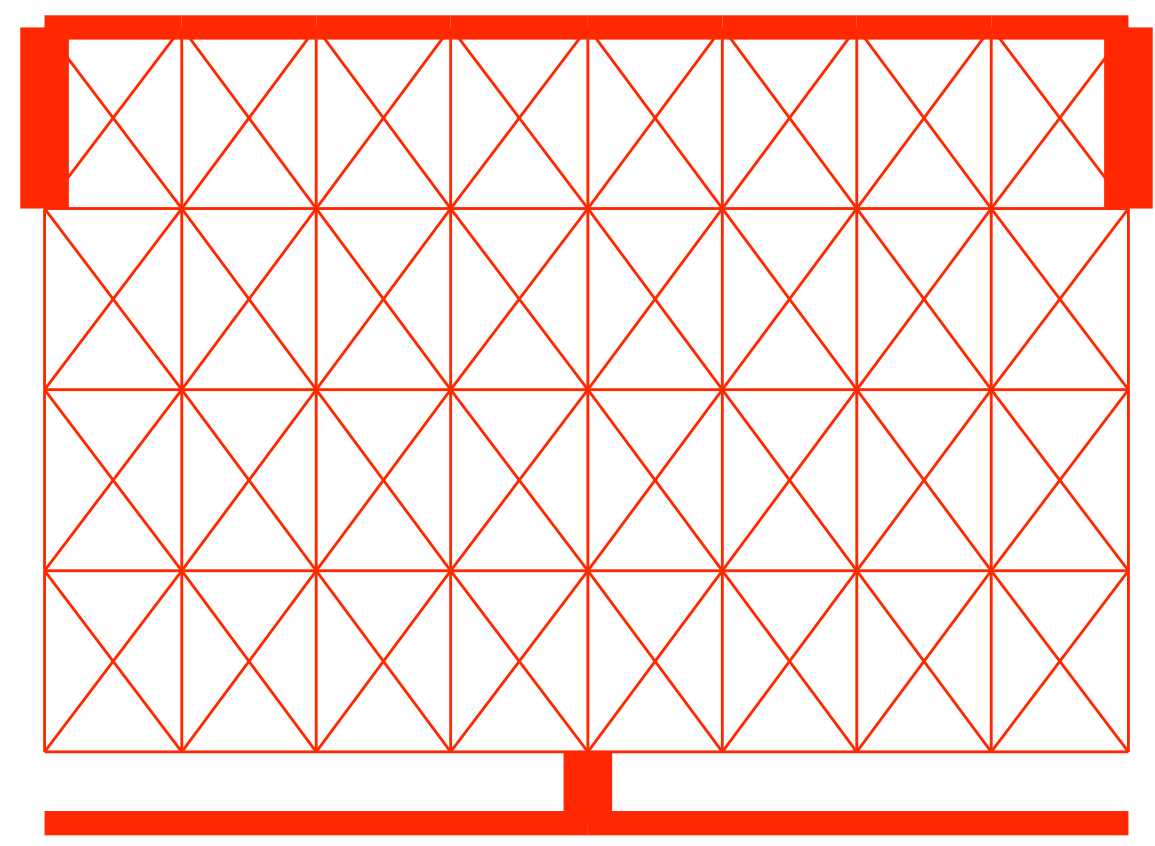

Figure 4.8: Initial sizing of unit cell binary ground structure (thickest lines have a width of $5.0 \mathrm{~mm}$; intermediate thickness lines have a width of $2.5 \mathrm{~mm}$; thinnest lines have a width of $0.5 \mathrm{~mm}$ )

iterated over 10 generations. Selection, crossover, and mutation probabilities were set at $80 \%, 80 \%, 1 \%$, respectively. These same probabilities were used to compute the optimum topologies presented in the remaining examples in this chapter.

The unit cell was optimized to minimize the FV over the reduced frequency interval of $2320-2330 \mathrm{~Hz}$ at frequency steps of $10 \mathrm{~Hz}$ and $n=2$ in Eqn (4.1). After execution of the optimization routine, the top layer RMS transverse velocity response of each top ten initial unit cell topology was computed from $0 \mathrm{~Hz}$ to $10 \mathrm{kHz}$, again evaluated in $10 \mathrm{~Hz}$ frequency steps. The filtering routine was then applied to this ten member subset of the final generation to obtain the filtered unit cells.

A filter threshold of $0.6 \mathrm{~mm}$ was used in determining the final ten unit cell optimum topologies. All members within the optimization domain of a specific unit cell 
that had a width less than $0.6 \mathrm{~mm}$ were reassigned a thickness of $2.54 \mathrm{E}-5 \mathrm{~mm}$, which from a computational standpoint represents a negligible stiffness. The top layer RMS transverse velocity response of each filtered unit cell was then re-computed from 0-10 $\mathrm{kHz}$. Since the bottom layer thickness of the unit cell was held fixed at $2.5 \mathrm{~mm}$, the attenuation zone starting frequency for each unit cell is $2320 \mathrm{~Hz}$ as determined using Eqn (3.16). The filter threshold was set by determining the maximum number of frame elements that could be eliminated from the optimization domain while maintaining reasonable breadth of the first attenuation zone and connectivity between the unit cell bottom layer, core members, and top layer.

The final ten optimum topologies are shown in Figures $4.9(\mathrm{a})-(\mathrm{j})$, where thinner lines represent frame elements that have greater compliance. Missing elements have a width below the filter threshold and are removed for visual clarity of the underlying topology. Both the initial and filtered top layer RMS transverse velocity responses for each unit cell are shown in Figures 4.10 (a)-(j). With the exception of optimum topology eight, the filtering routine maintains the breadth of the first attenuation zone. However, observe that the filtering routine introduces a greater number of resonant peaks just after the cutoff frequency for the first attenuation zone and prior to the start of the second attenuation zone. In this numerical example no disconnected unit cell designs were obtained, although two designs have hanging or floating elements (e.g. optimum topology four and seven), Figure 4.9.

\subsubsection{Results: Example 2}

Since the initial population from which the optimization routine proceeds is randomly generated, different runs can produce different results depending on the number of individuals in a population and the number of generations used. Accordingly, in this second example the results from another optimization run are provided. A 

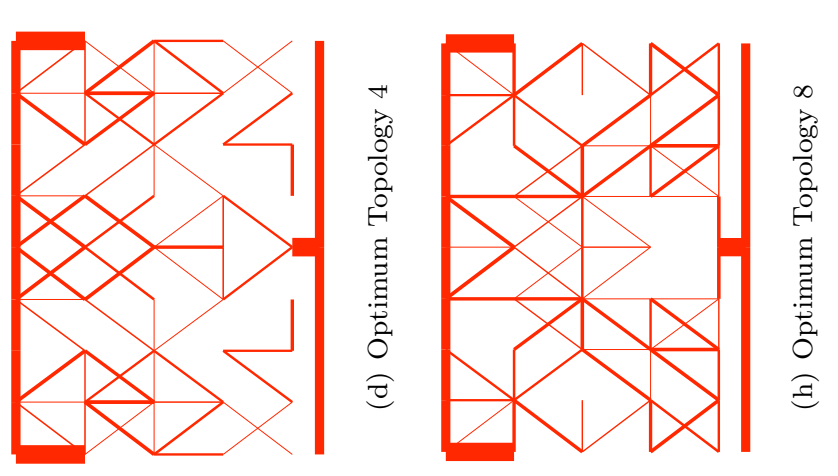

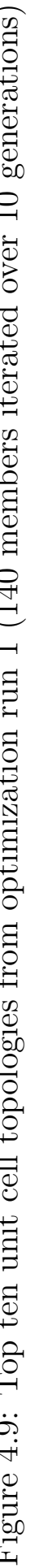



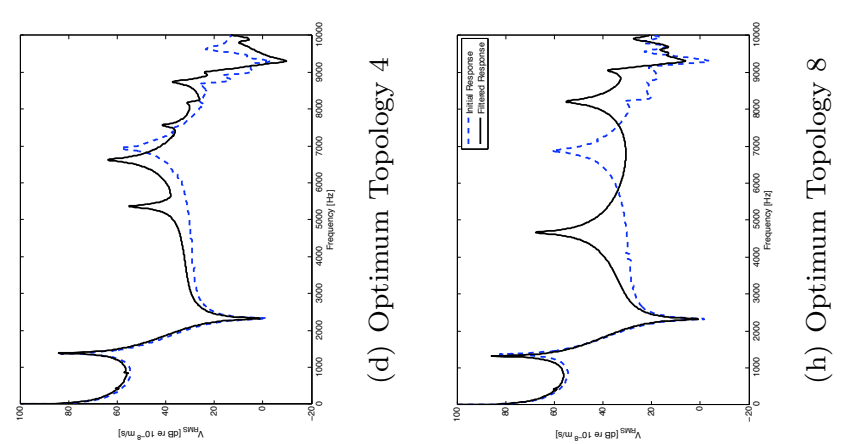

0
0
0
0
0
0
0
0
0
0
0
0
0
0
0
0
0
0
0
0
0
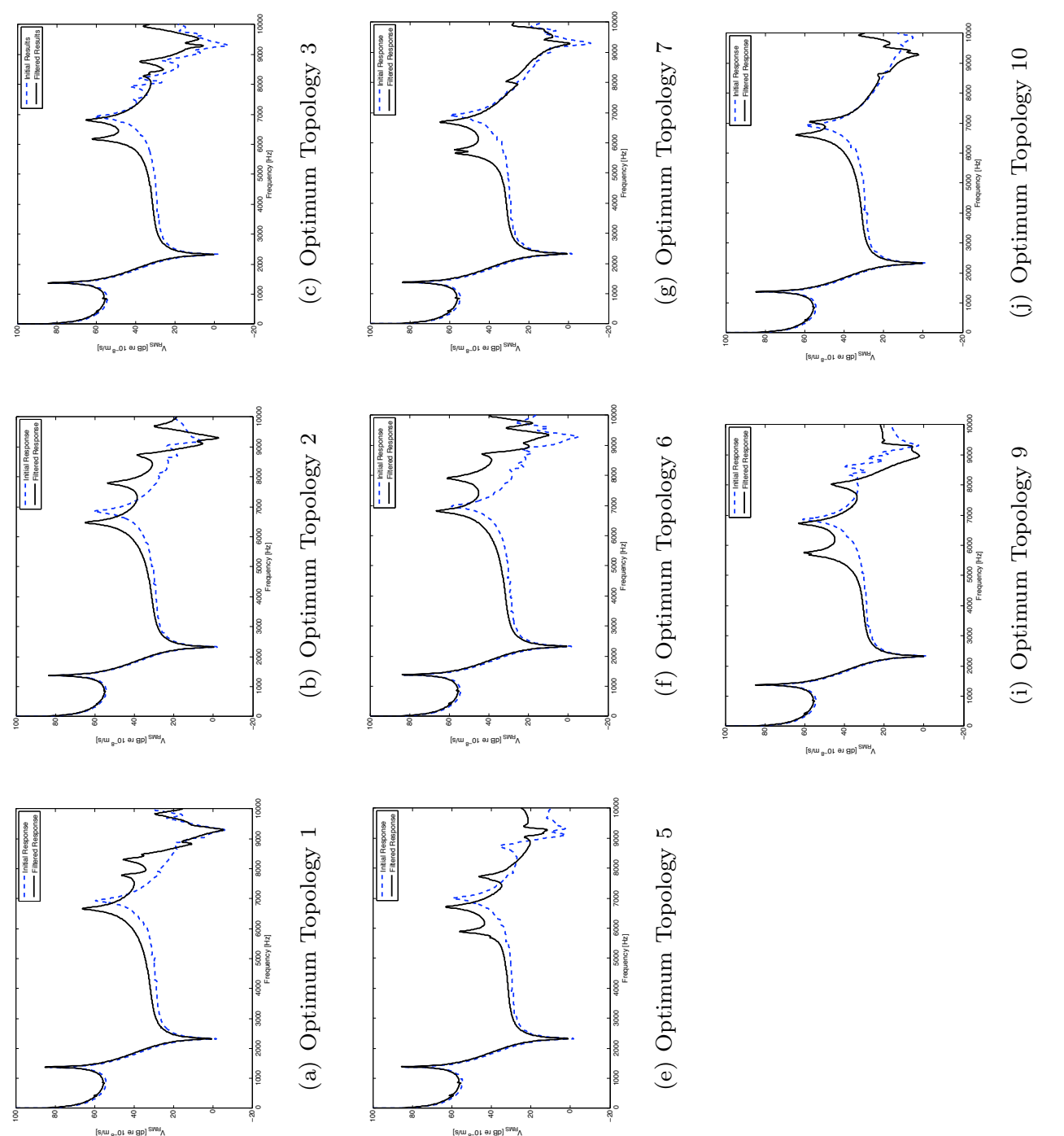


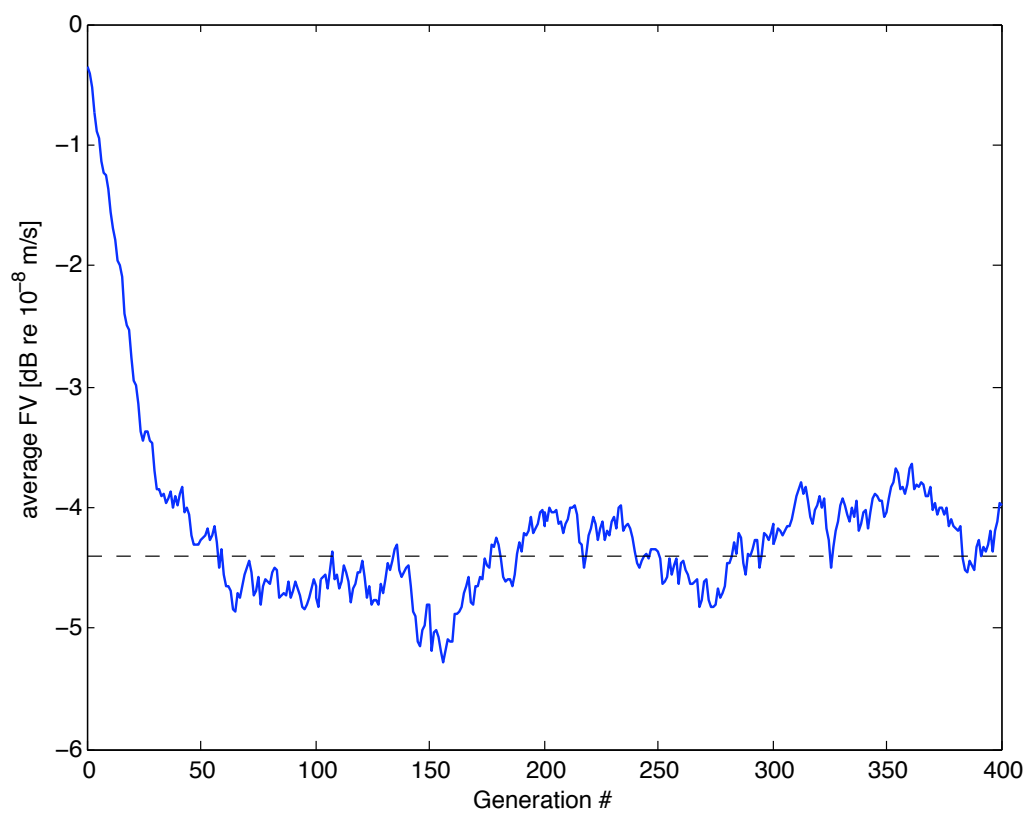

Figure 4.11: Average FV of 400 member population over 400 generations

population size consisting of 400 members was iterated over 400 generations. To further explore convergence of the genetic algorithm the average FV of each generation was monitored. The average FV is shown as a function of generation number in Figure 4.11. This figure demonstrates that the average FV of the population has converged after approximately 65 generations. The drift in the average FV after this point is due to random variations introduced by mutation as discussed by Stoffa \& Sen [94]. Beyond generation 65 the average of the remaining average fitness values is -4.4 dB. Further inspection of Figure 4.11 reveals that despite this drift, the genetic algorithm consistently trends back towards the average value of $-4.4 \mathrm{~dB}$, as expected.

Given the final generation, a subset consisting of the top ten optimum unit cell topologies was selected and the top layer RMS transverse velocity response of each selected initial unit cell design was computed over the entire $10 \mathrm{kHz}$ frequency range. The filtering procedure was then applied. In this example, a filter threshold of 0.7 

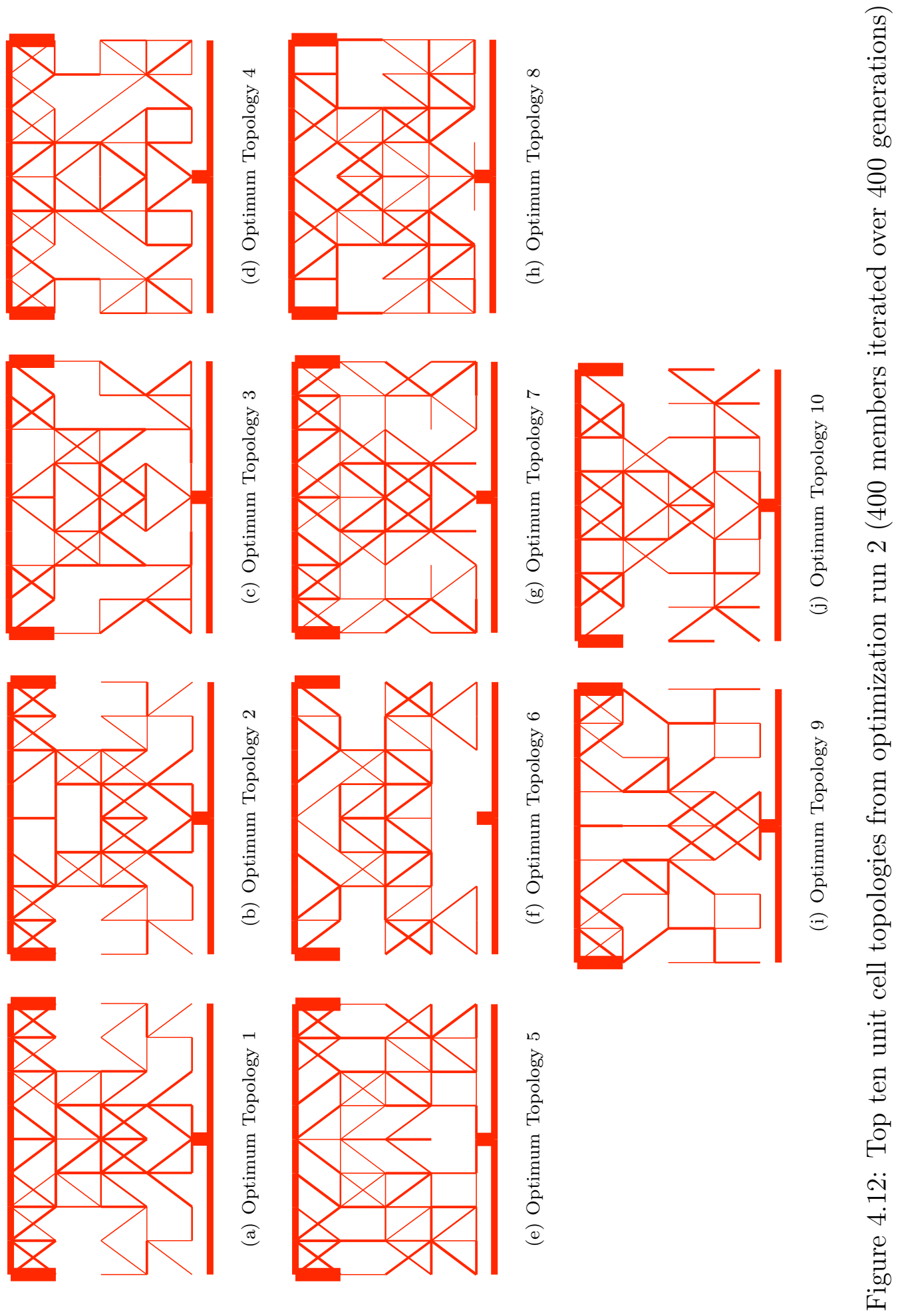

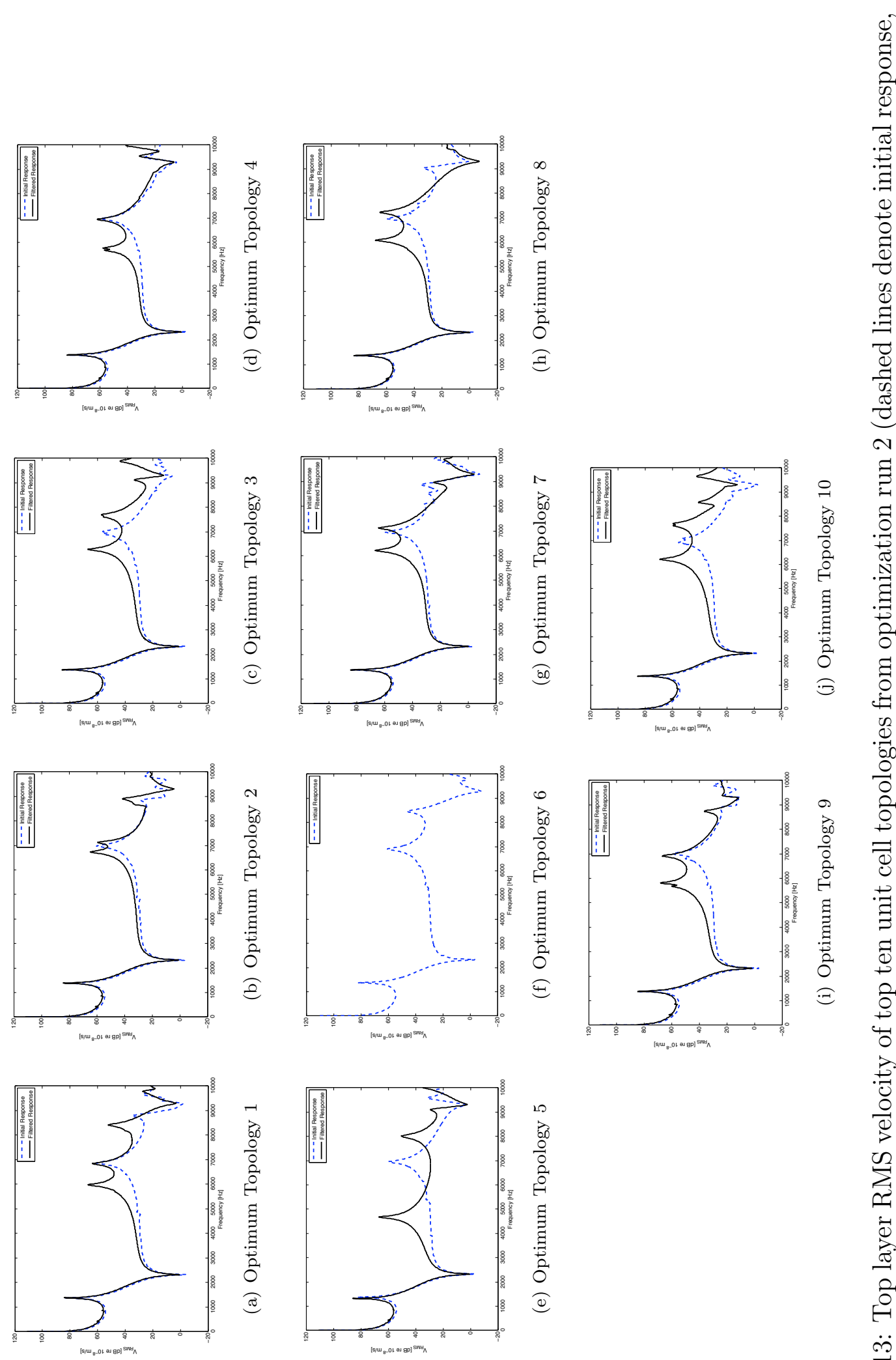

N

$\exists$

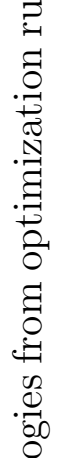

0
0
0
0
0
0
0
$\vdots$
0
0
0
0
0
0
0
0
0
0
0

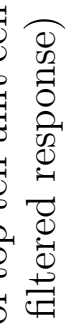

总葛

告

工

సั.

है

尚 
mm was used. The larger filter threshold value, compared to the first example from Section 4.2.2, was found to produce designs having maximum sparseness while maintaining attenuation zone breadth and unit cell connectivity. After the application of the filtering routine, the top layer RMS transverse velocity response of each unit cell design was computed over the $10 \mathrm{kHz}$ frequency range and compared with the respective initial response.

The final ten optimum topologies are shown in Figures $4.12(\mathrm{a})-(\mathrm{j})$, where thinner lines represent frame elements with greater compliance. The elements that are missing from these figures have a width below the $0.7 \mathrm{~mm}$ filter threshold. The initial and filtered top layer RMS transverse velocity responses for each optimized unit cell are shown in Figures $4.13(\mathrm{a})-(\mathrm{j})$. Observe that using a larger filter threshold value produced only one disconnected design (i.e. optimum topology six). Similar to the first numerical example from Section 4.2.2, the filtering routine introduces a greater number of resonant peaks between the first and second attenuation zones. In the case of optimum topology five the filtering routine substantially modifies structural dynamic response with the result being that the first attenuation zone is significantly shortened.

\subsubsection{Results: Example 3}

In this final example the results from a third optimization run are given. Optimization was performed using all of the same analysis parameters from the first example in Section 4.2 .2 (e.g. 140 members, 10 generations, $0.6 \mathrm{~mm}$ filter threshold value, etc...). From the top ten unit cell designs, only the third best was selected for further investigation. The selected optimized topology is shown in Figure 4.14 and it was chosen due to its relative sparseness. The objective behind studying this specific topology in-depth is to determine whether a simplified compliant mechanism 


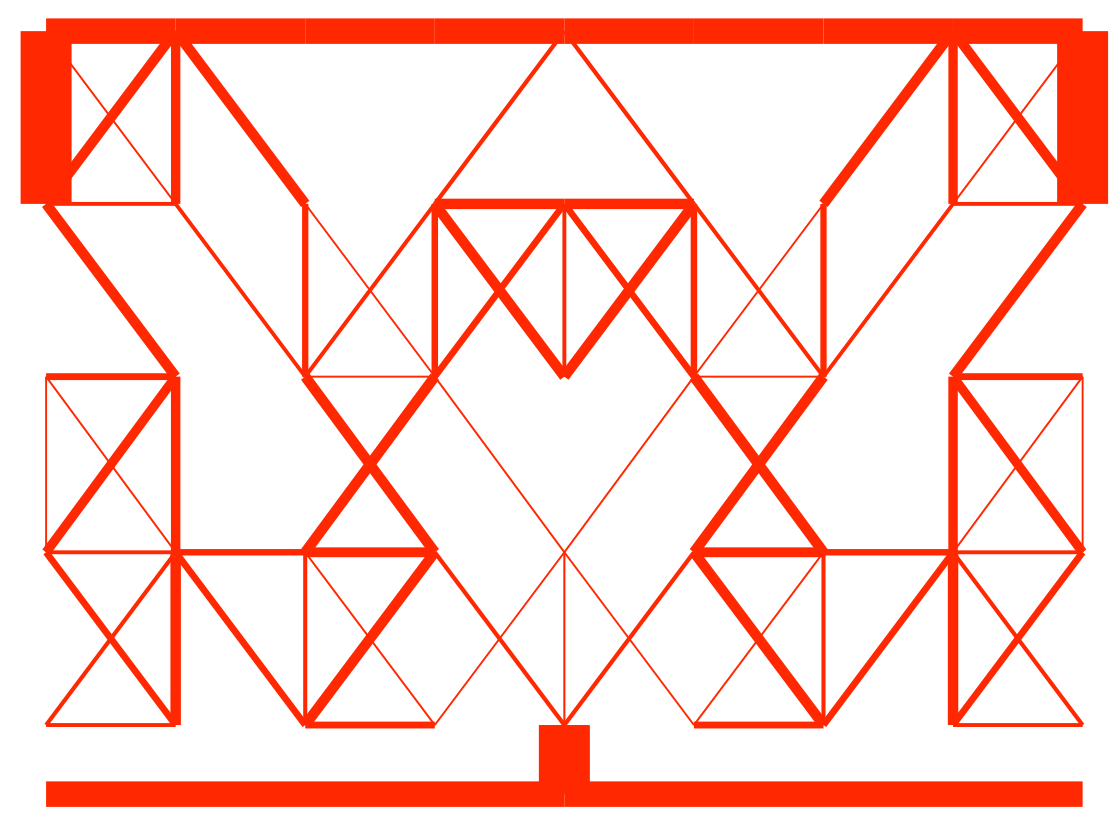

Figure 4.14: Third best unit cell topology from optimization run 3 (140 members iterated over 10 generations)

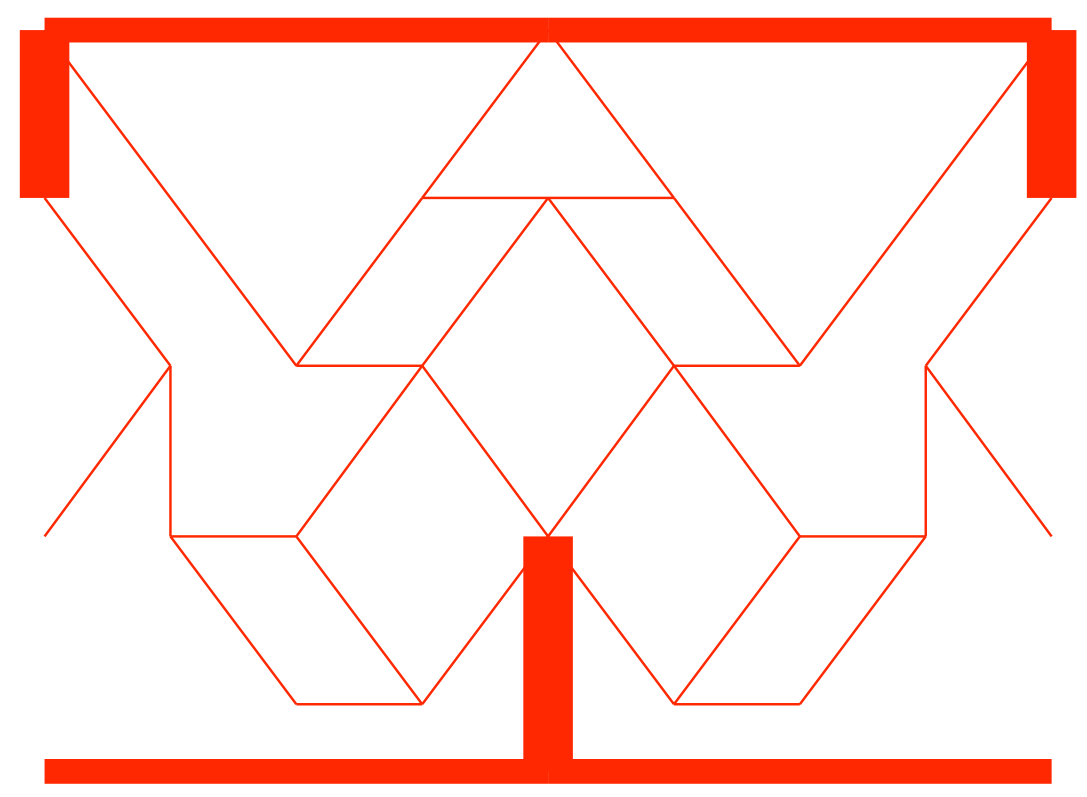

Figure 4.15: Reduced unit cell topology (thickest lines have a width of $5.0 \mathrm{~mm}$; intermediate thickness lines have a width of $2.5 \mathrm{~mm}$; thinnest lines have a width of $0.6 \mathrm{~mm}$ ) 
unit cell design that has further reduced geometric complexity can be derived.

The filtered unit cell topology, Figure 4.14, was utilized as a template from which elements were manually removed to obtain a "reduced unit cell" topology. This reduced topology is shown in Figure 4.15. Element thicknesses were determined by using the filtered unit cell topology as an approximate sizing guide. The top and bottom layer width remains fixed at $2.5 \mathrm{~mm}$. For simplicity, all unit cell core members were given a uniform thickness of $0.6 \mathrm{~mm}$. The remaining transmission and ground members were assigned the largest width of $5.0 \mathrm{~mm}$. Observe that the transmission member that connects the unit cell bottom layer to the core members has been extended. Additionally, members have been eliminated such that the reduced unit cell contains no remaining members that cross over each other, thus establishing a physically realizable configuration.

The computed RMS transverse velocity responses of the initial unit cell, the filtered unit cell, and the reduced unit cell are shown in Figure 4.16. Examining the dynamic response of the reduced unit cell reveals that the breadth of the first attenuation zone remains the same while amplitude increases slightly (i.e. approximately $5 \mathrm{~dB})$ at the beginning of the zone. In general, greater modal density is observed at frequencies below $8 \mathrm{kHz}$, which is explained by examining the reduced unit cell FRF. Above $9 \mathrm{kHz}$ the amplitude of response within the second attenuation zone has increased by approximately $15 \mathrm{~dB}$.

The reduced unit cell FRF is computed by taking the input as the transverse velocity of the bottom layer center point (i.e. node 13 in Figure 4.17) relative to the ground point. The output is taken at the transverse velocity of the top layer center point (i.e. node 16) relative to the ground point. The motion of the ground point is assumed to be represented by the transverse velocity of top layer left corner 


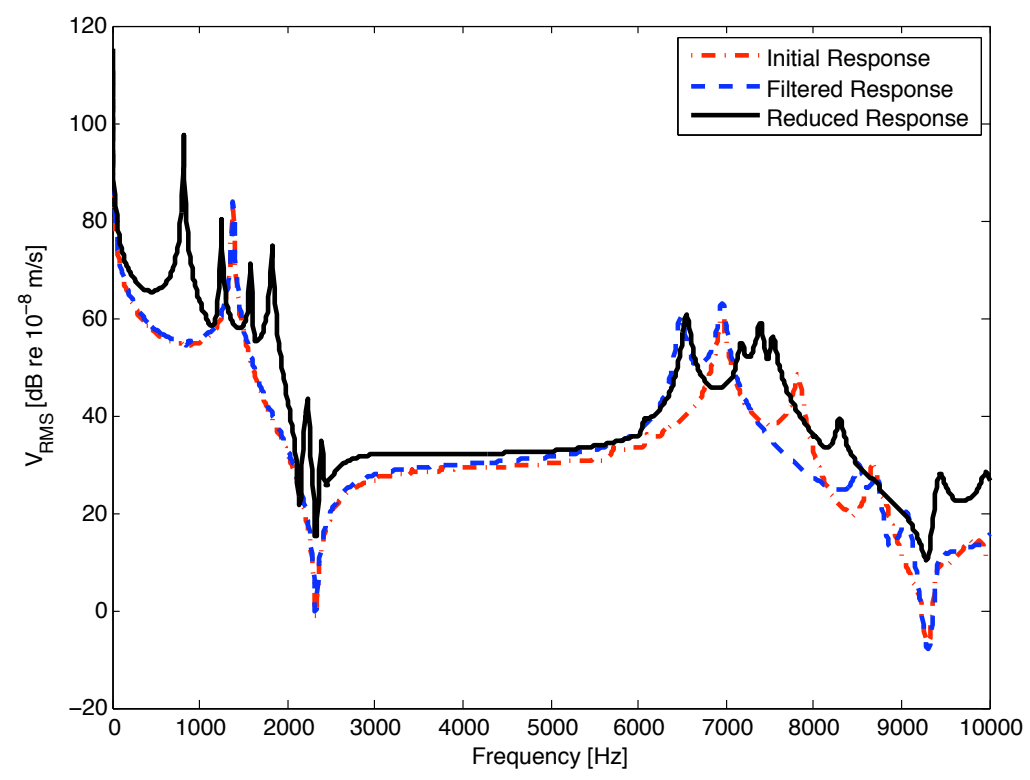

Figure 4.16: Top layer RMS velocity response of initial, filtered, and reduced unit cell

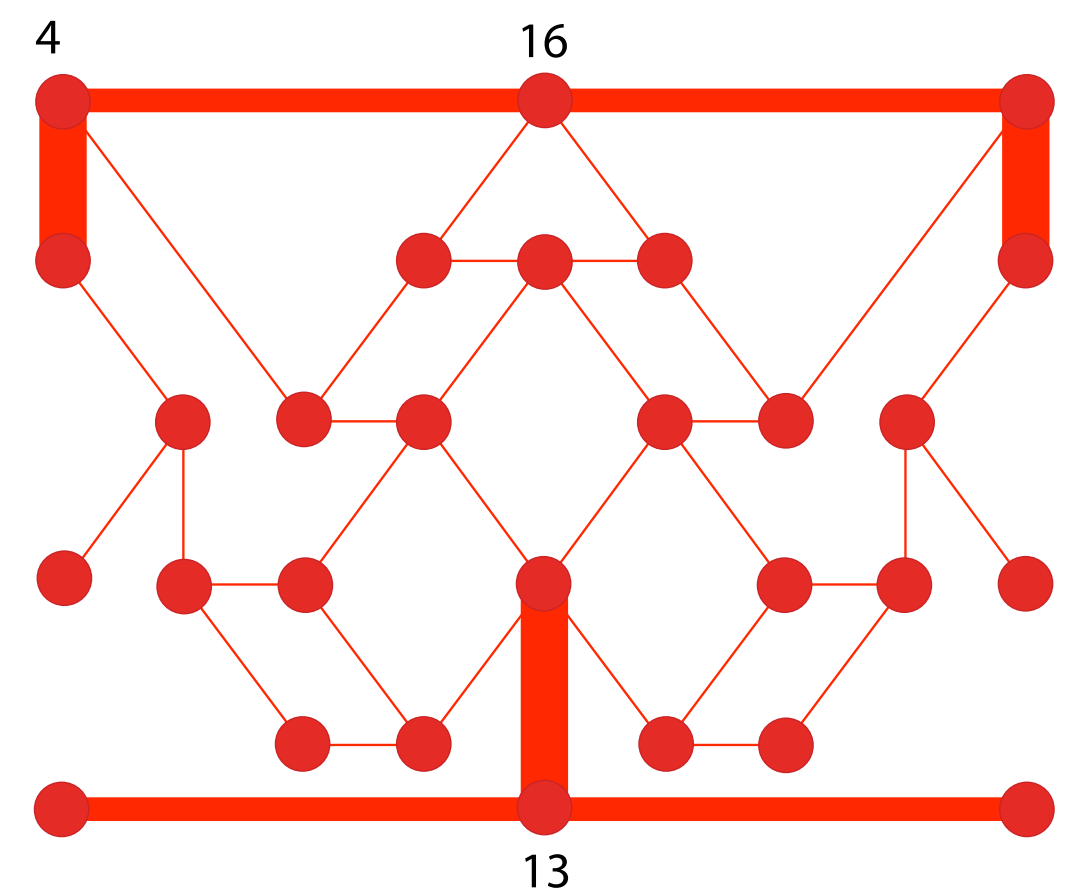

Figure 4.17: Reduced unit cell model (numbers denote nodes) 


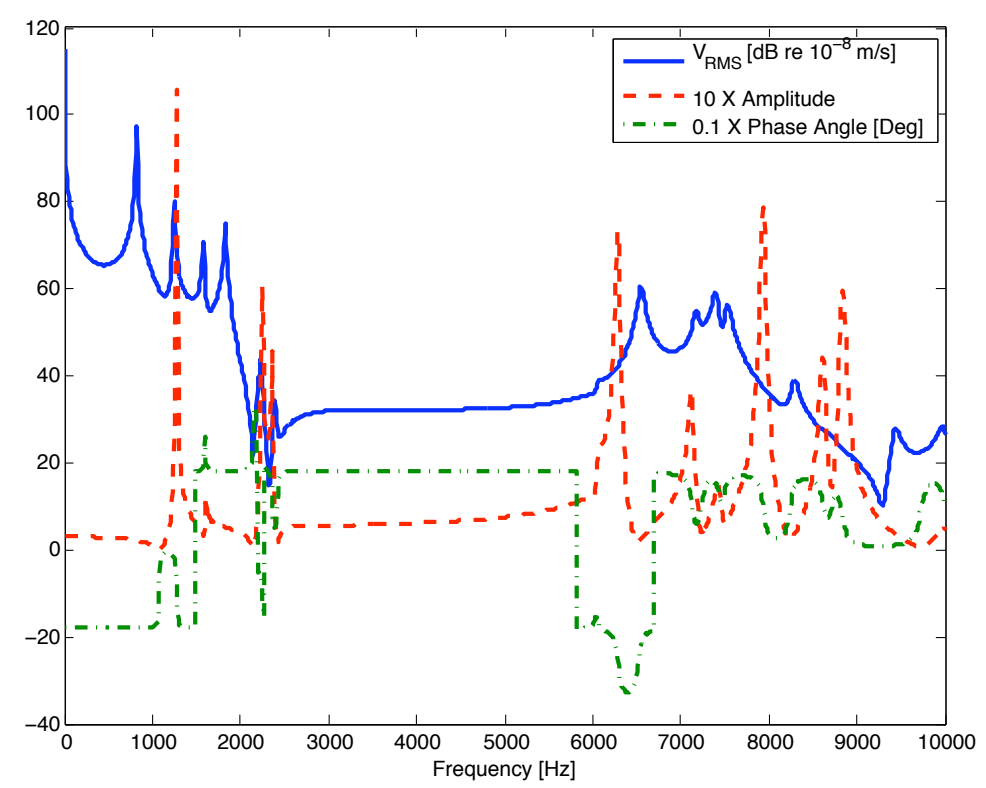

Figure 4.18: FRF of reduced unit cell topology

of the unit cell (i.e. node 4). The reduced unit cell FRF shown in Figure 4.18 exhibits resonant peaks that are characterized by larger amplitudes and excursions of the compliant mechanism phase angle away from 180 degrees. Observe that inphase, lower amplitude response implies the familiar "inversor" type of deformation characteristic within the first attenuation zone.

Accordingly, to better visualize the "inversor" behavior of the integral compliant mechanism the deformed shape and total energy distribution of the reduced unit cell topology is shown at three frequencies in Figures 4.19 through 4.21. In these figures line thickness denotes energy content. At $4000 \mathrm{~Hz}$, Figure 4.19, bottom layer energy isolation and out-of-phase deformation characterizes dynamic response. In contrast, energy transmission through the unit cell core to the top layer occurs at $6550 \mathrm{~Hz}$, Figure 4.20, despite continued out-of-phase motion. Similarities between the deformed shape of this reduced unit cell topology and the deformed shape of 


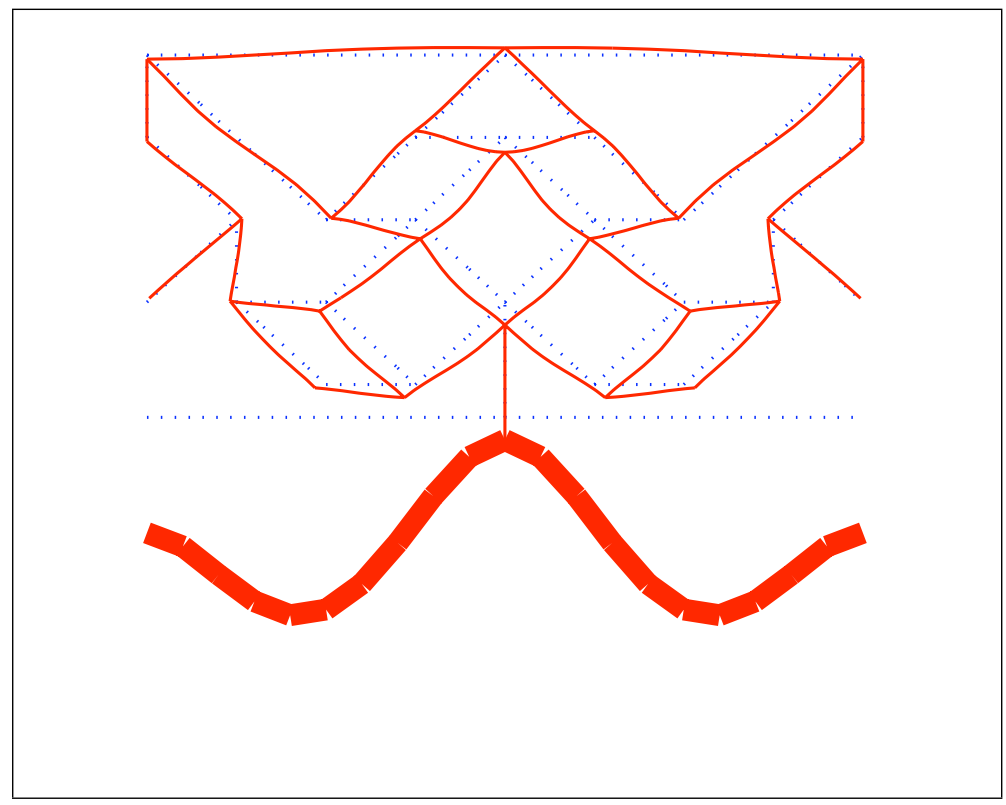

Figure 4.19: Reduced compliant mechanism unit cell deformed shape and energy distribution at $4000 \mathrm{~Hz}$ (dotted lines denote undeformed configuration, continuous lines denote deformed configuration)

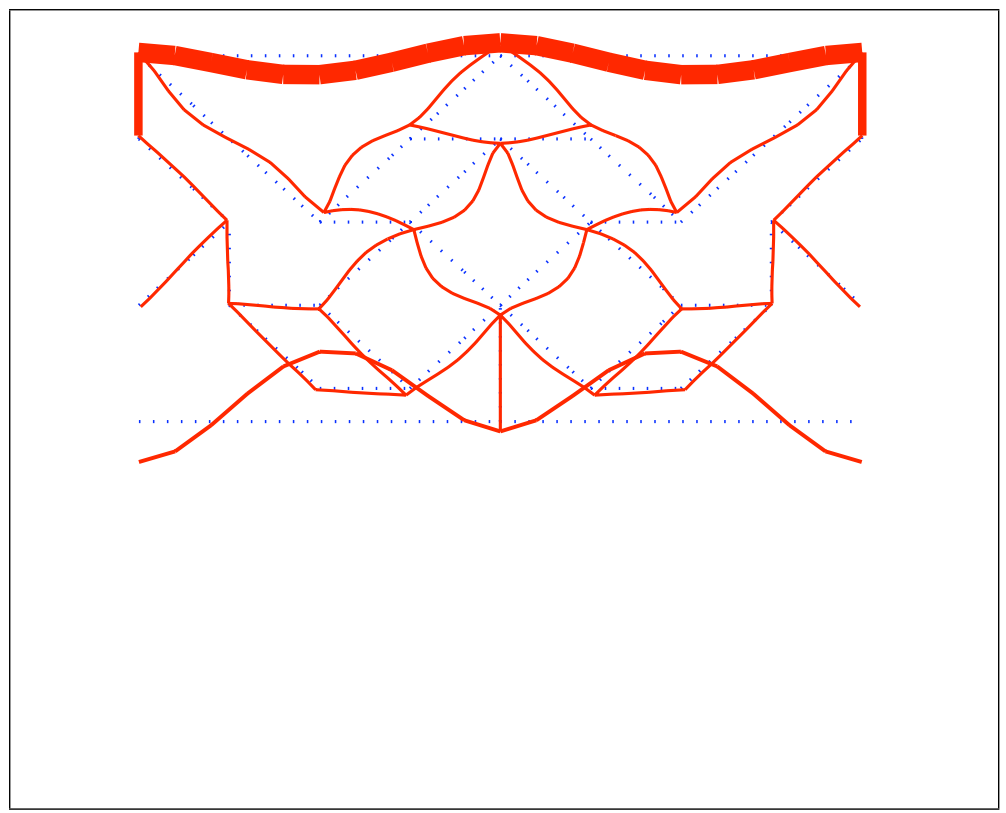

Figure 4.20: Reduced compliant mechanism unit cell deformed shape and energy distribution at $6550 \mathrm{~Hz}$ (dotted lines denote undeformed configuration, continuous lines denote deformed configuration) 


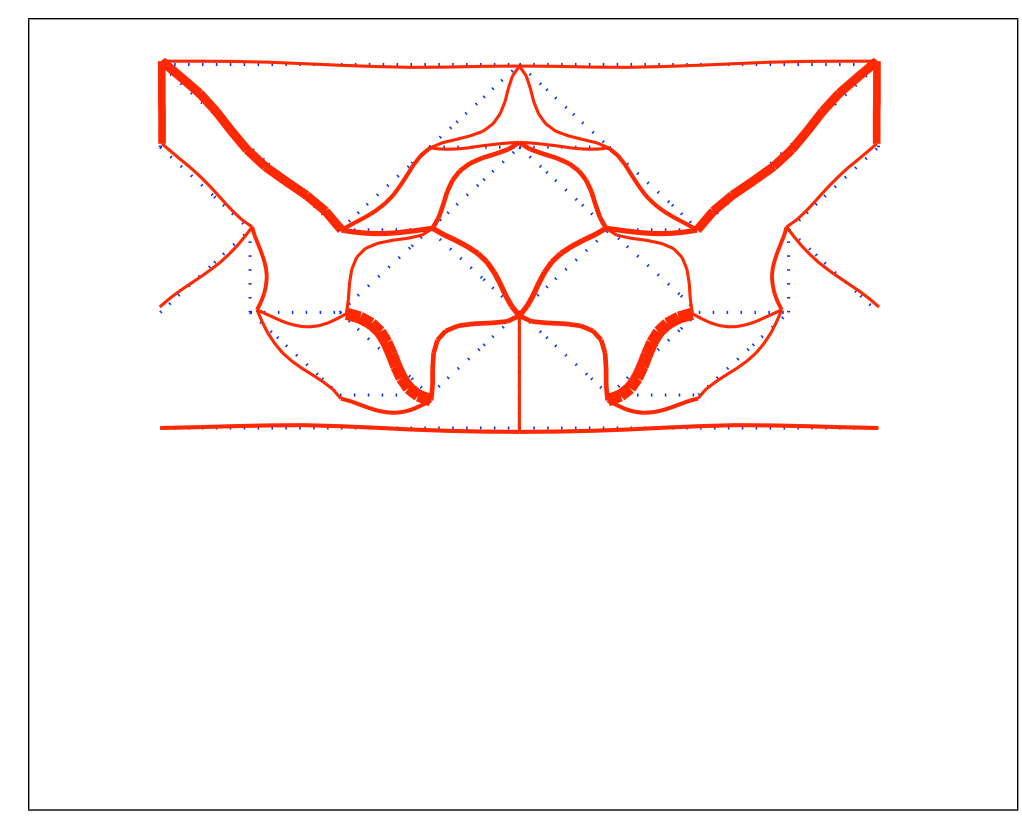

Figure 4.21: Reduced compliant mechanism unit cell deformed shape and energy distribution at $9240 \mathrm{~Hz}$ (dotted lines denote undeformed configuration, continuous lines denote deformed configuration)

the rhombus compliant mechanism unit cell topology are clearly evident at these two frequencies. In contrast, unique response is seen at $9240 \mathrm{~Hz}$, Figure 4.21, where in-phase deformation occurs and the majority of the total energy content is isolated within the core of the unit cell rather than the bottom layer. Nonetheless, the limited energy transmission to the top layer of the unit cell explains the reduced amplitude of the RMS normal velocity response.

This dynamic behavior illustrates that alternative compliant mechanism unit cell designs that are obtained via the topology optimization routine are likely to exhibit dynamic performance that differs somewhat from that of the other unit cell topologies presented in this research and obtained using a deductive approach. However, the assemblage of computational tools presented to this point continues to provide sufficient insight into the physical nature of the structural dynamic response. 


\subsubsection{Discussion}

In order to compare the reduced unit cell design (Section 4.2.4) with the original compliant mechanism unit cell design (Section 3.2.1) and the rhombus compliant mechanism unit cell design (Section 3.2.4), the definition of mechanical advantage is revisited. Specifically, an alternative formula for the mechanical advantage of the unit cell, MA $\mathrm{UNIT}_{\text {UCELL }}$, is defined by taking the input speed, $\mathrm{v}_{\text {in }}$, in Eqn (3.13) as the RMS transverse velocity of all unit cell bottom layer nodes and the output speed, $\mathrm{v}_{\text {out }}$, as the RMS transverse velocity of all unit cell top layer nodes.

$$
\mathrm{MA}_{\text {UNIT_CELL }}=\frac{\sqrt{\left(1 / N_{\text {bot }}\right) \sum_{i=1}^{N_{\text {bot }}}\left|\hat{v}_{i}^{\text {bot }}\right|^{2}}}{\sqrt{\left(1 / N_{\text {top }}\right) \sum_{j=1}^{N_{\text {top }}}\left|\hat{v}_{j}^{\text {top }}\right|^{2}}}
$$

In Eqn (4.2) the bottom layer transverse spectral displacements are summed over the number of bottom layer nodes, $N_{\text {bot }}$. The top layer transverse spectral displacements are summed over the number of top layer nodes, $N_{\text {top }}$.

The log of the mechanical advantage is computed using Eqn (4.2) and is shown as a function of frequency in Figure 4.22 for the three compliant mechanism unit cell designs. The first compliant mechanism unit cell design (Section 3.2.1) exhibits the largest mechanical advantage over the majority of the $10 \mathrm{kHz}$ frequency range. The second (i.e. rhombus) compliant mechanism unit cell design (Section 3.2.4) presents a roughly comparable mechanical advantage within the first attenuation zone, however, demonstrates generally lower levels above $7 \mathrm{kHz}$. The reduced unit cell exhibits the lowest mechanical advantage within the first attenuation zone. Despite this, the first attenuation zone cutoff frequency for the reduced unit cell is increased by approximately $1500 \mathrm{~Hz}$ when compared with the other two designs. Above $7 \mathrm{kHz}$ the reduced unit cell demonstrates mechanical advantage values that are comparable with the mechanical advantage values of the rhombus compliant mechanism unit cell. 


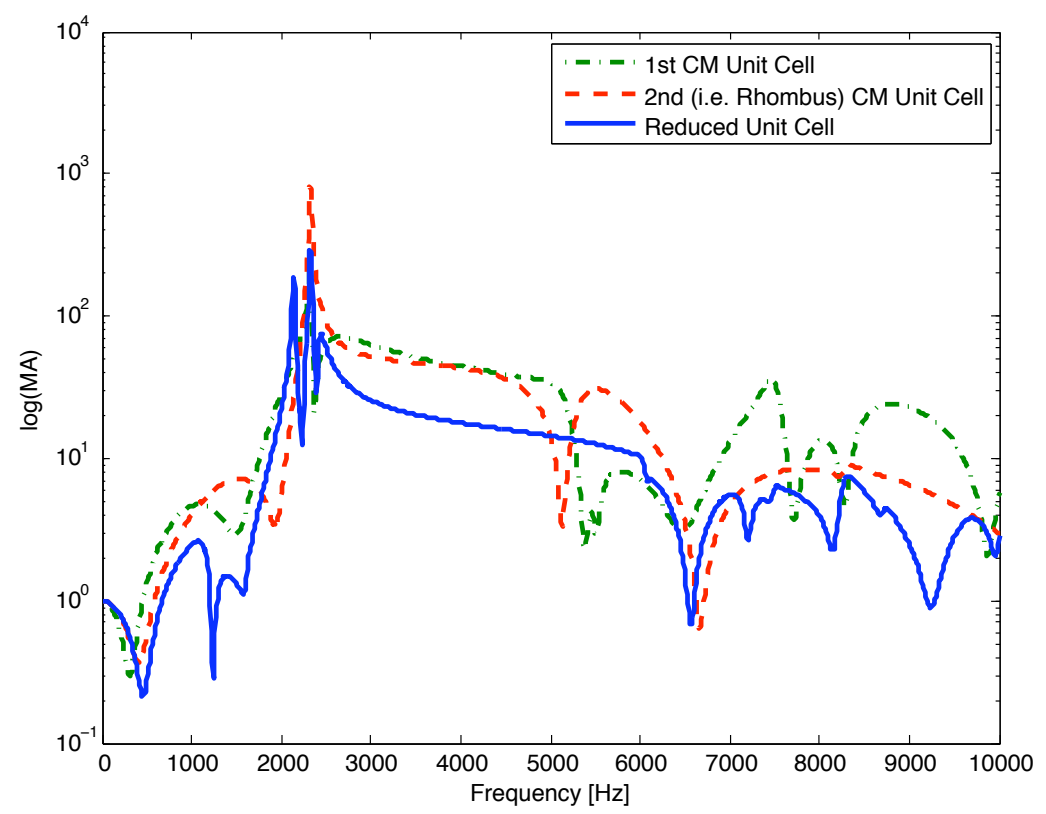

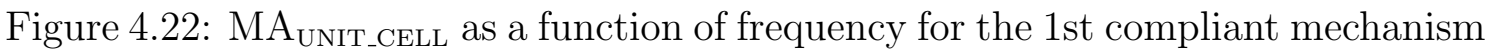
unit cell (Section 3.2.1), the 2nd (i.e. rhombus) compliant mechanism unit cell (Section 3.2.4), and the reduced unit cell

As a final point, inspection of the initial unit cell binary ground structure from Figure 4.6 reveals that the topologies of the original two compliant mechanism unit cell designs presented in Section 3.2 are not contained within the possible set of outcomes defined by this starting optimization domain. This represents a significant limitation of the method proposed in this chapter. Thus, while satisfactory results have been obtained using the established optimization procedure and simple network structure, it is likely that enhanced solutions can be arrived at through the implementation of a more complex optimization domain. Furthermore, a more sophisticated genetic algorithm code could also be developed to implement the load path representation method proposed by Lu \& Kota [61]. An advantage of the load path method is that it does not require an initial discretization network. Thus, intuition or prior experience is not required to determine the complexity or configuration 
of a starting mesh.

Despite these drawbacks, the genetic algorithm optimization method outlined in this chapter provides a realistic starting point for further study. The results presented in this chapter highlight alternative means of computing compliant mechanism unit cell topologies that have specific dynamic functionality exclusive of a priori knowledge of a preferred design. 


\section{CHAPTER 5}

\section{Verification \& Validation}

To validate the computational results presented in the prior two chapters, experimental investigations were undertaken. Specifically, two structures were manufactured and tested in order to validate their computed mid-frequency structural dynamic response. The first structure has a conventional square core that is patterned after the design presented in Section 3.1. This structure functions as a test control for comparison purposes. The second structure is a compliant mechanism prototype structure that has a rhombus compliant mechanism core that is patterned after the design given in Section 3.2.4.

The design of the compliant mechanism prototype is set forth in Section 5.1 and includes: the prototype concept, definitions, spectral finite element computational analysis, and results. Descriptions of the manufacturing and experimental test setup for the prototype and conventional square core structures are given in Sections 5.2 and 5.3, respectively. In Section 5.4 the normal velocity response of each structure, measured using a non-contacting laser vibrometer, is reported and compared with the results from the initial spectral finite element computational analysis. A conventional two-dimensional finite element computational analysis is also introduced in Section 5.5 for complete verification of the experimentally obtained structural dynamic response. A discussion of the experimental study is given in Section 5.6. 


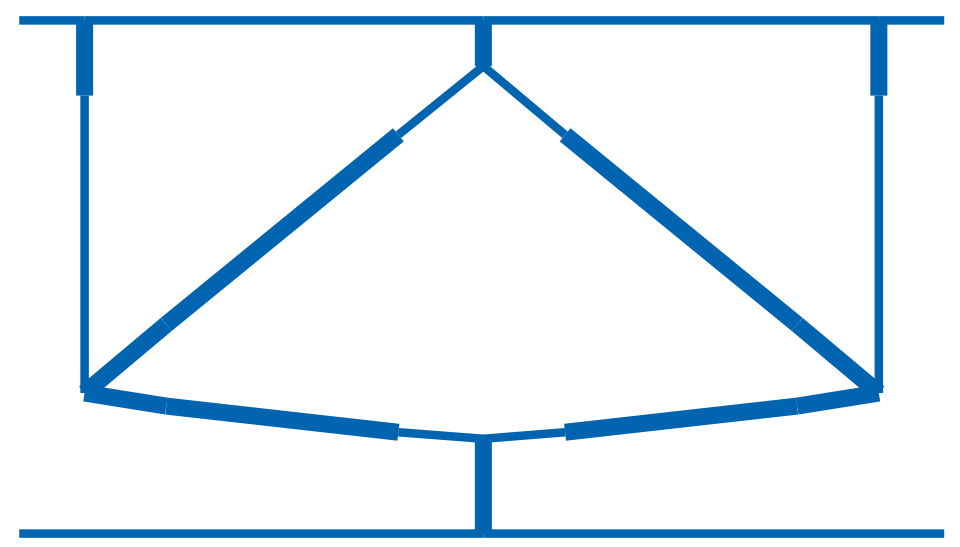

Figure 5.1: Rhombus compliant mechanism unit cell model

\subsection{Prototype Design}

The rhombus compliant mechanism unit cell topology is used as a template for this work. Minor modifications to the sizing of the unit cell are made in order to ensure manufacturability of the final prototype structure. The initial computational analysis is performed using one-dimensional linear spectral finite elements. Thus, this analysis establishes the baseline performance of the prototype compliant mechanism unit cell and structure prior to experimental study.

\subsubsection{Prototype Concept, Definitions, \& Spectral Element Analysis}

The rhombus compliant mechanism unit cell model used in this investigation is shown in Figure 5.1. This model is similar to the one shown in Figure 3.26 with a few modifications to the unit cell sizing and individual member sizing. The conceptual model of a rhombus compliant mechanism structure, which is an assemblage of six unit cells, is given in Figure 5.2. The structure is fixed to a rigid baffle at both ends and is subjected to a localized distributed load induced by an incident pressure wave.

Since the unit cell model in Figure 5.1 is the same as the one in Section 3.2.4 


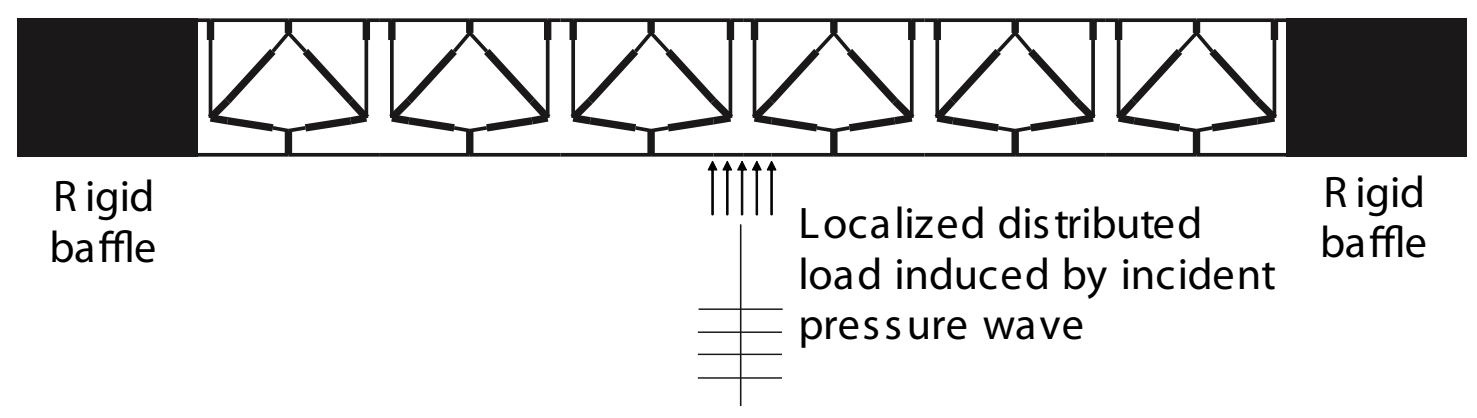

Figure 5.2: Periodic rhombus compliant mechanism structure

(exclusive of the physical sizing) all of the analytical relationships previously derived (e.g. the FRF) are still valid. Likewise, the details of the computational analysis follows the approach first introduced for the square core unit cell and structure in Chapter 3 .

\subsubsection{Computational Results: Spectral Elements}

In accordance with the manufactured structures, the unit cell and structure are assumed to be made of 6061-T6 aluminum with a material Young's modulus, $E=$

$6.83 \times 10^{10} \mathrm{~N} / \mathrm{m}^{2}$, and density, $\rho=2713.6 \mathrm{~kg} / \mathrm{m}^{3}$, per the Military Standardization Handbook: MIL-HDBK-5 [15]. Hysteretic material damping is introduced to account for inherent energy loss. A complex modulus, $E^{*}=E(1+i \eta)$, is utilized with $\eta=$ 0.01 .

In Figure 5.1, thicker lines represent stiffer elements having a width of $5 \mathrm{~mm}$. 
Thinner lines represent elements $2.5 \mathrm{~mm}$ wide. The sizing of members within the compliant mechanism unit cell is consistent with the sizing of members in the conventional square core unit cell. Recall that the square core unit cell top and bottom facesheet width is taken as $5 \mathrm{~mm}$ and core members are assigned a thickness of 2.5 $\mathrm{mm}$, per Section 3.1.2.

Given the bottom layer physical parameters (i.e. $\rho, E, A$, and $I$ ), the overall compliant mechanism unit cell length (which is set equal to the wavelength, $\lambda$, of vibration) is determined using the wavenumber-frequency relationship, Eqn (3.16), along with a selected attenuation zone starting frequency of $2320 \mathrm{~Hz}$. Accordingly, unit cell dimensions of $0.1 \mathrm{~m}$ by $0.055 \mathrm{~m}$ are used, leading to a compliant mechanism with a mechanical advantage of 7.0. The out-of-plane depth of the unit cell is set to $0.0127 \mathrm{~m}$. These dimensions dictate a structure that is $0.6 \mathrm{~m}$ long, $0.055 \mathrm{~m}$ wide, and $0.0127 \mathrm{~m}$ deep in size.

A pressure wave amplitude, $p_{\text {in }}=1 \mathrm{~N} / \mathrm{m}^{2}$, is used in calculating the equivalent nodal loading, Eqn (3.7), for the unit cell analysis. The rhombus compliant mechanism unit cell FRF and top layer RMS transverse velocity, evaluated from $0 \mathrm{~Hz}$ to $10 \mathrm{kHz}$ at $10 \mathrm{~Hz}$ frequency steps, is given in Figure 5.3. Below $2320 \mathrm{~Hz}$, two peaks in response are visible at $1100 \mathrm{~Hz}$ and $2110 \mathrm{~Hz}$. Above $2320 \mathrm{~Hz}$, two major attenuation zones are present spanning the frequency ranges of $2320-5300 \mathrm{~Hz}$ and $9240-9500 \mathrm{~Hz}$. At the starting frequencies of these attenuation zones, the wavelength of vibration for transverse waves in the unit cell bottom layer is equal to the unit cell width and half-width (i.e. $\lambda=0.1 \mathrm{~m}$ and $\lambda=0.05 \mathrm{~m}$ ), respectively, per Eqn (3.16). Within each attenuation zone the mechanism demonstrates out-of-phase, non-resonant (i.e. small amplitude) behavior, as expected. Between the attenuation zones (i.e. 5300$9240 \mathrm{~Hz}$ ) in-phase, resonant behavior is exemplified by peaks in the RMS transverse 


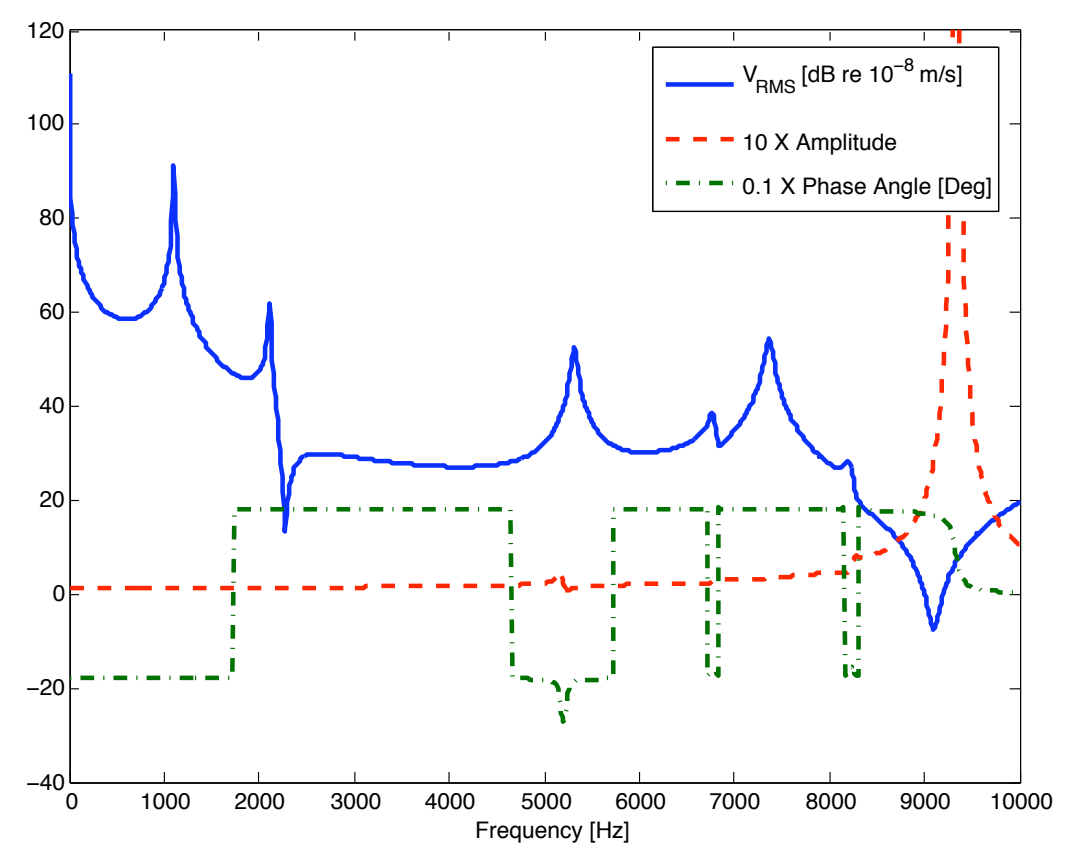

Figure 5.3: Rhombus compliant mechanism unit cell FRF

velocity response, increases in the FRF amplitude, and a FRF phase that transitions away from 180 degrees. Similar increases in response are visible immediately after the second attenuation zone starting frequency of $9240 \mathrm{~Hz}$. Just after this frequency, the RMS normal velocity response increases slightly, however a more prevalent peak in the FRF amplitude is present in conjunction with a FRF phase angle that moves from out-of-phase (i.e. 180 degrees) towards in-phase (i.e. zero degrees).

Moving to a global analysis of the structure, an equivalent nodal loading is computed based on a localized SPL amplitude of $145.4 \mathrm{~dB}$ re 20E-6 Pa. From this point forward all SPL values are assumed to be calculated using a reference pressure level of 20E-6 Pa unless stated otherwise. This equivalent localized forcing is assumed to act over a $0.025 \mathrm{~m}$ by $0.0127 \mathrm{~m}$ area centered about the middle of the bottom layer of the structure (refer to Figure 5.2). The RMS transverse velocity of the compliant mechanism structure, computed using the response from 23 top layer nodes (i.e. $N$ 


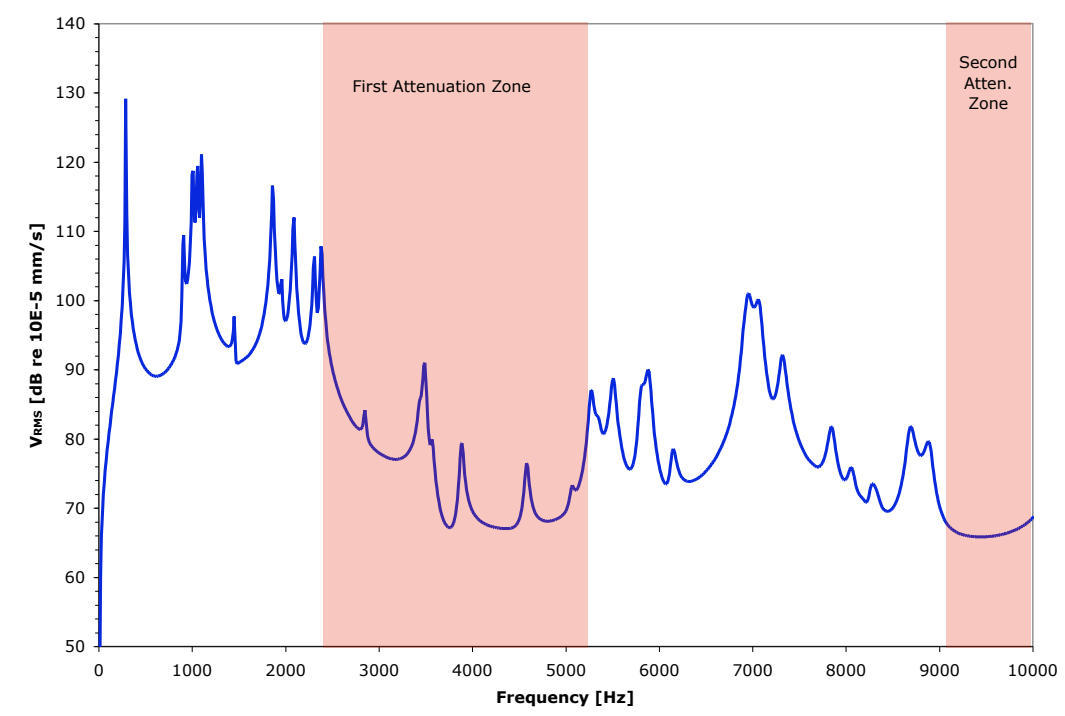

Figure 5.4: Top layer RMS velocity (computed using the response from 23 top layer nodes) for compliant mechanism structure

$=23)$ in Eqn (3.9), is given in Figure 5.4. The first global resonance of the structure occurs at $230 \mathrm{~Hz}$. Unit cell resonances at $1100 \mathrm{~Hz}$ and $2110 \mathrm{~Hz}$ are visible in the response of the structure despite increased modal density. The first attenuation zone exists between approximately $2300 \mathrm{~Hz}$ and $5300 \mathrm{~Hz}$ with the occurrence of four smaller amplitude resonant peaks. Between $5 \mathrm{kHz}$ and $9 \mathrm{kHz}$ structural dynamic response increases as expected, and above $9 \mathrm{kHz}$ the second attenuation zone is visible. The minor resonances within the first attenuation zone highlight the inability of the unit cell analysis in predicting the interaction between multiple unit cells. Nonetheless, given the overall breadth of the $10 \mathrm{kHz}$ frequency range, the general structural dynamic characteristic of interest is verified at the global level.

\subsection{Prototype Manufacturing}

Prior to manufacturing each structure the respective solid model geometry was generated using a commercial CAD software package, I-DEAS. The solid model ge- 


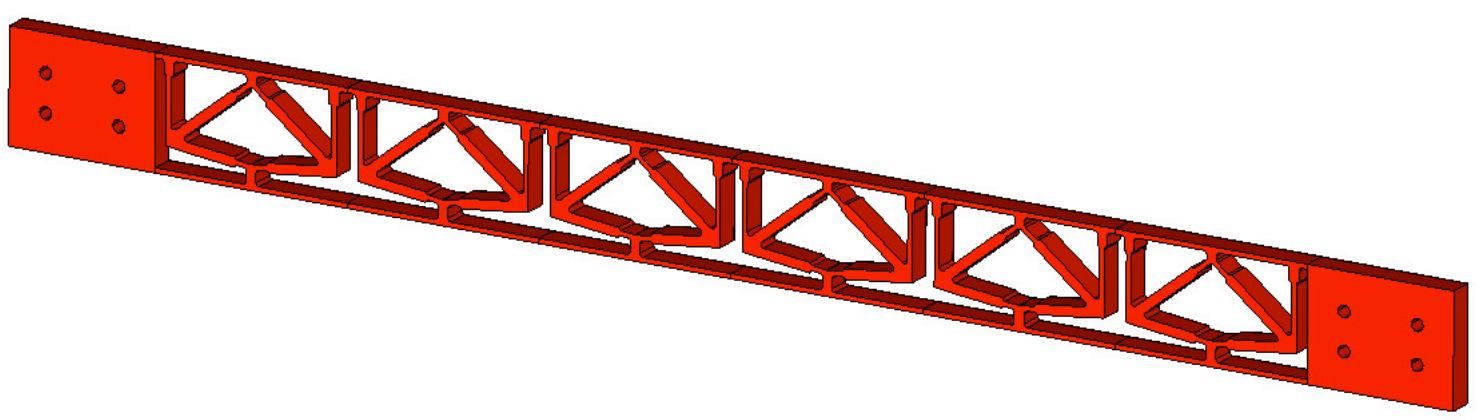

Figure 5.5: Solid model geometry of rhombus compliant mechanism structure

ometry of the prototype rhombus compliant mechanism structure is shown in Figure 5.5. The bolt holes located on both ends of the prototype provide the means by which the structure is rigidly attached to the experimental test fixture.

A standard 3-axis CNC mill was used to manufacture the prototype rhombus compliant mechanism and conventional square core structures, as shown in Figure 5.6. The structures were produced by exporting the CAD solid model geometry to .IGS files and importing those files into commercial CAM software, GibbsCAM, to generate the machining tool paths for the milling operations. The pocket geometry was machined using a 1/8-inch diameter end mill as shown in Figures 5.7 (a) and (b).

\subsection{Prototype Testing}

The experimental test setups for the compliant mechanism structure and the square core structure are shown in Figures 5.8 and 5.9, respectively. Fixed-fixed boundary conditions were enforced by mounting each end of each structure to a steel standoff, which was then rigidly attached to an optical bench. Each test setup was designed to minimize inadvertent excitation of torsional and out-of-plane bending modes. 


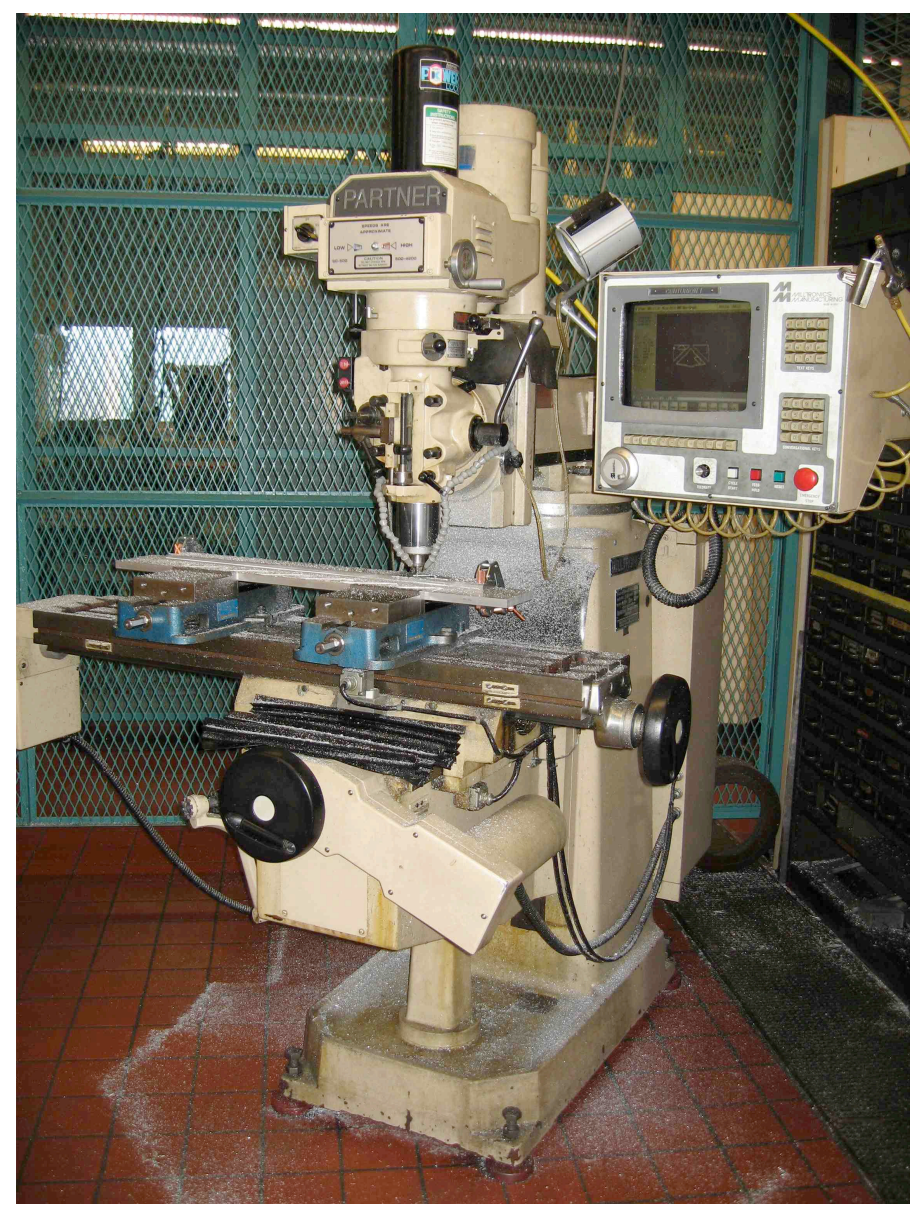

Figure 5.6: Machining of compliant mechanism structure on 3-axis CNC mill

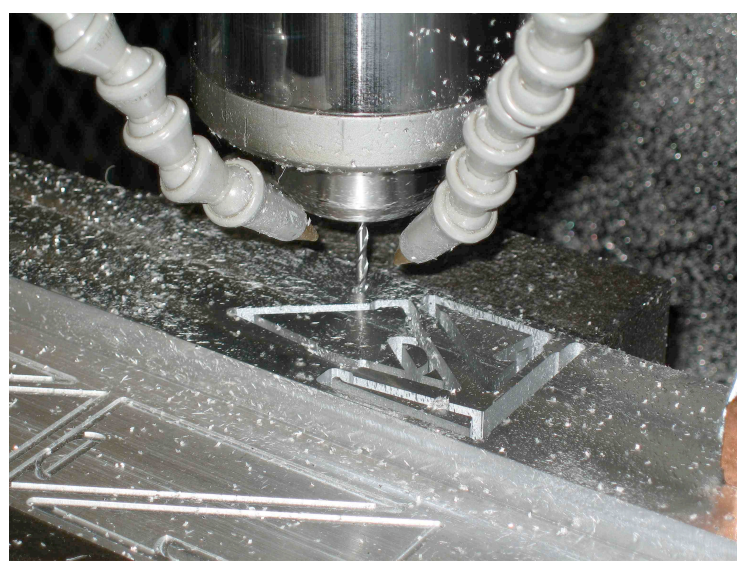

(a) Pocket geometry

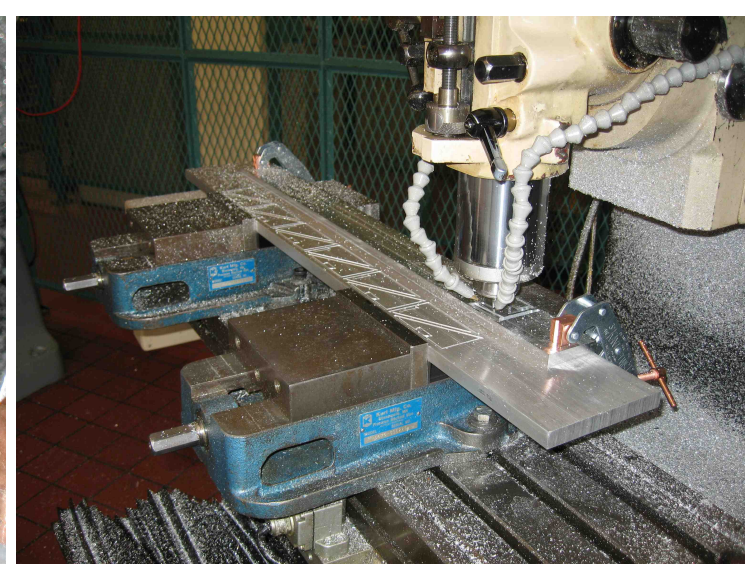

(b) Machine fixturing

Figure 5.7: Machining of pocket geometry for compliant mechanism structure using 1/8-inch diameter end mill: (a) pocket geometry and (b) machine fixturing 


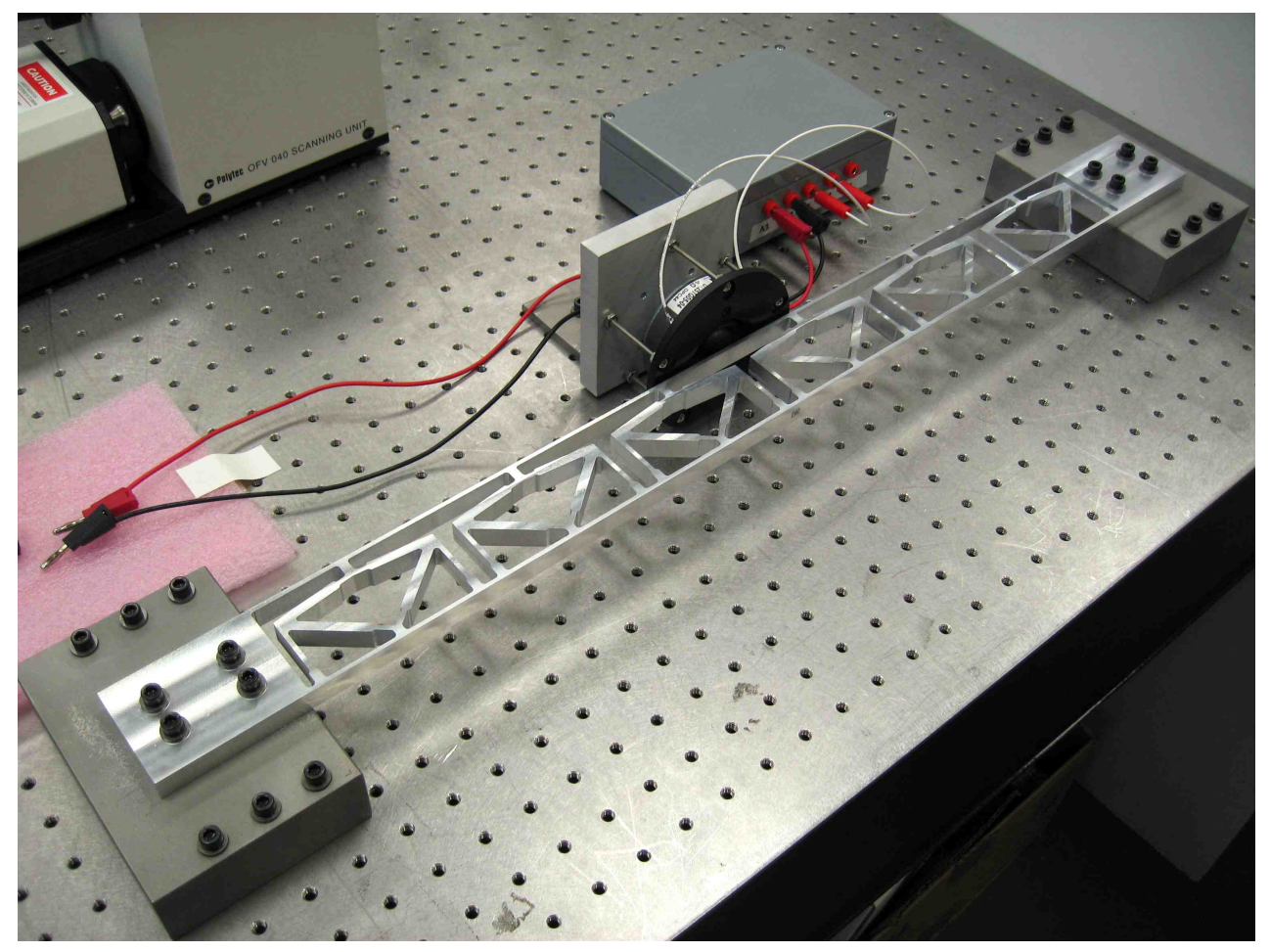

Figure 5.8: Experimental test setup for compliant mechanism structure

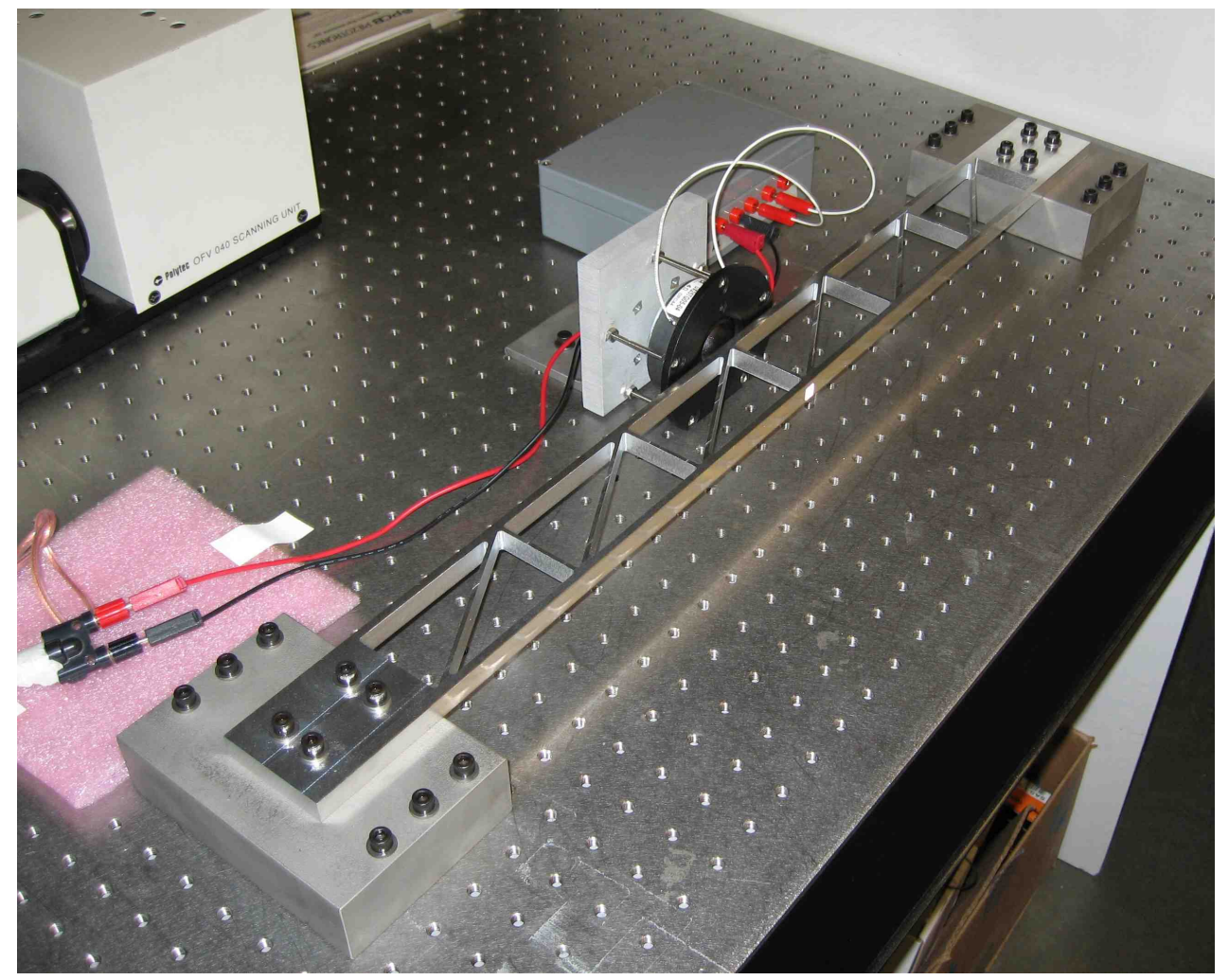

Figure 5.9: Experimental test setup for square core (test control) structure 


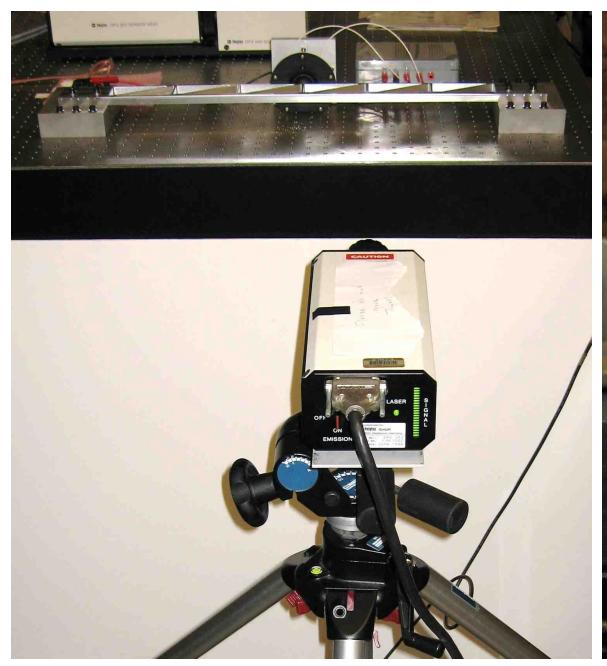

(a) SPLV

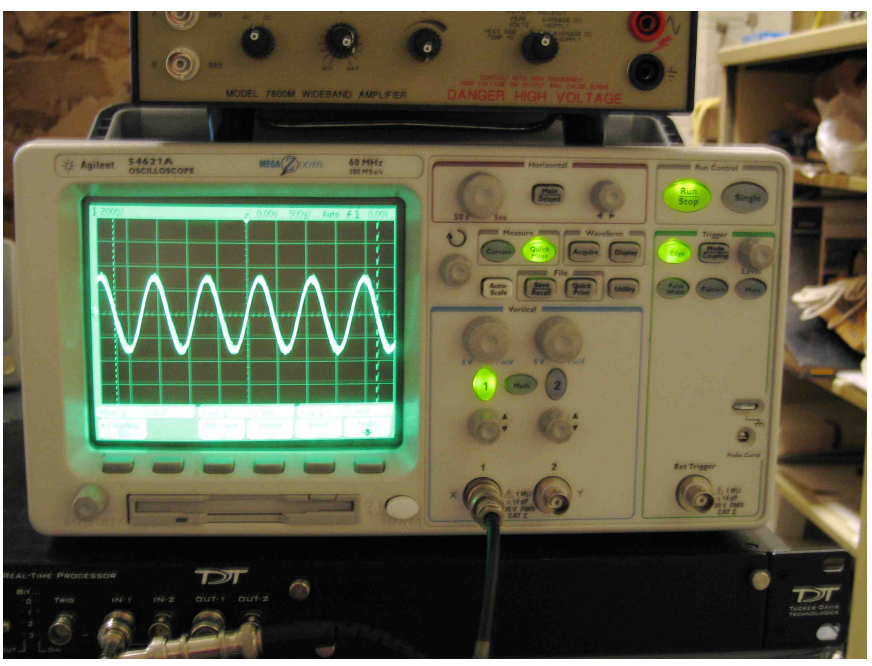

(b) Oscilloscope

Figure 5.10: Experimental measurement system: (a) single point laser vibrometer (SPLV) and (b) oscilloscope

Appendix B contains a schematic of the acoustic excitation system, the high pass filter design, and details regarding the use of the non-contacting SPLV measurement system. Non-contacting localized excitation of each structure was achieved using a $0.025 \mathrm{~m}$ diameter tweeter, VIFA model DX25TG05-04, positioned $0.64 \mathrm{~mm}$ from the center of the structure bottom layer. The diameter of the loudspeaker was selected to match the length over which the localized distributed load was applied in the computational analysis. Care was taken to assure the loudspeaker diaphragm did not touch the structure during testing. A first order (i.e. $6 \mathrm{~dB}$ per octave) high pass filter with a crossover frequency of $500 \mathrm{~Hz}$ was connected in series with the loudspeaker to allow pure harmonic stepped-sine excitation in the frequency range of $1 \mathrm{kHz}$ to 10 $\mathrm{kHz}$ at $10 \mathrm{~Hz}$ frequency intervals. A standard PC with LabVIEW software and an analog output channel were used as a signal generator. The sinusoidal signal was then sent to the loudspeaker via an amplifier, Adcom model GFA-535, and the high pass filter. Response was measured using a non-contacting single point laser vibrometer 
(SPLV), Polytec model OFV-2602 with OFV-353 optics, shown in Figure 5.10 (a). A total of 901 data points were taken per measurement location for each structure. The SPLV output voltage was measured using an oscilloscope, Agilent model 54621A, per Figure 5.10 (b). The oscilloscope output voltage was then converted to a velocity using the appropriate SPLV scale factor. Sufficient dwell time between frequency steps was taken to ensure that transient response had decayed significantly so that only steady state response was dominant.

Two measurement locations were monitored for each structure. The RMS transverse velocity of the compliant mechanism structure is a composite of the transverse velocity at two points including the top layer center point and a point $0.05 \mathrm{~m}$ left of center. These two measurement locations were selected since they represent the point of maximum displacement and a compliant mechanism output node, respectively. The top layer center point and a point $0.1 \mathrm{~m}$ left of center were used for the square core structure. These measurement locations represent the point of maximum displacement and a point exactly one bay to the left, respectively.

Prior to testing, the loudspeaker was calibrated in an anechoic chamber by measuring the SPL with a Larson-Davis 2520 microphone and 2200C preamplifier. The speaker was driven with $8 \mathrm{~W}$ of power, and SPL measurements were taken at a distance of $1 \mathrm{~m}$ over a frequency range of $1 \mathrm{kHz}$ to $10 \mathrm{kHz}$ at $100 \mathrm{~Hz}$ intervals. As shown in Figure 5.11, scaling the measured SPL at $1 \mathrm{~m}$ to $0.64 \mathrm{~mm}$ allowed for the calculation of an input SPL to the structure of approximately $145.4 \mathrm{~dB}$. This allows for direct comparison of the experimentally measured response of each structure with the corresponding computational result. The sensitivity of the loudspeaker was such that a relatively flat response was obtained across the $1-10 \mathrm{kHz}$ test frequency range, Figure 5.11. This precludes concerns of large input pressure fluctuations, and hence, 


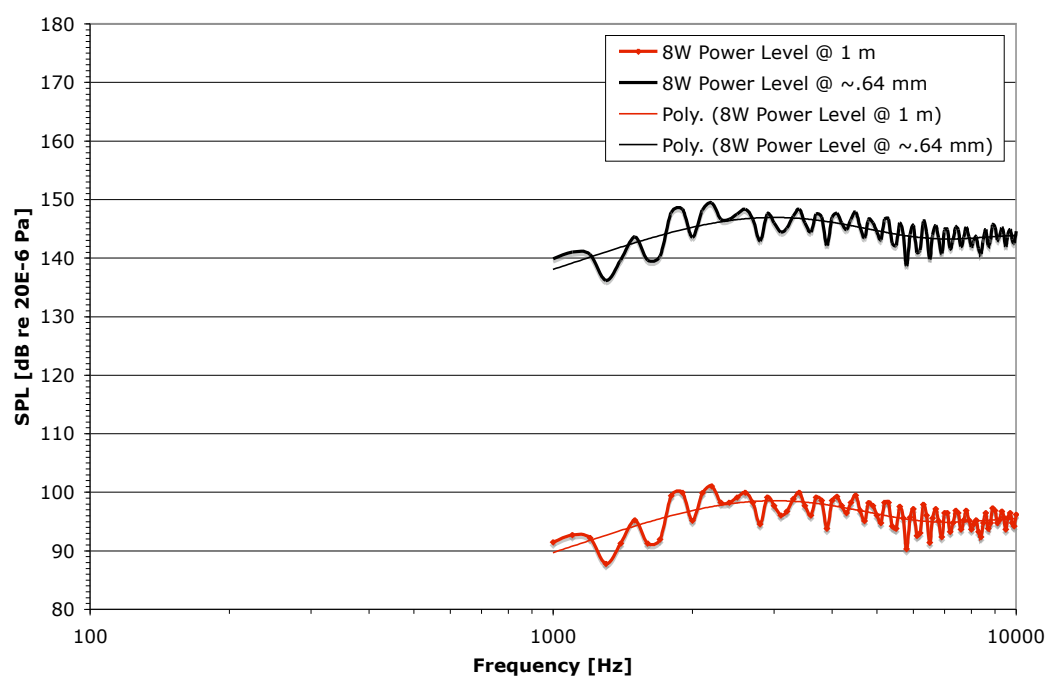

Figure 5.11: Speaker calibration SPL spectrum at $1 \mathrm{~m}$ scaled to $0.64 \mathrm{~mm}$

local forcing variation.

\subsection{Test Results}

In Figure 5.12 the RMS transverse velocity response of the square core structure, computed using the spectral finite element method, is compared with experimentally obtained results. For each computational result in this section the computed RMS transverse velocity is a composite of the normal velocity response at the same two top layer measurement locations, i.e. $N=2$ in Eqn (3.9), as described for the corresponding structure in the prior section. Similarly, Figure 5.13 shows the computational and experimental RMS transverse velocity response for the compliant mechanism structure.

Initial visual inspection of these figures reveals significant discrepancies. The experimental data for both structures exhibits greater modal density, larger resonant amplitudes, and a shifting of response towards higher frequencies. Specifically, the experimental data for the square core structure shows many more resonances below 


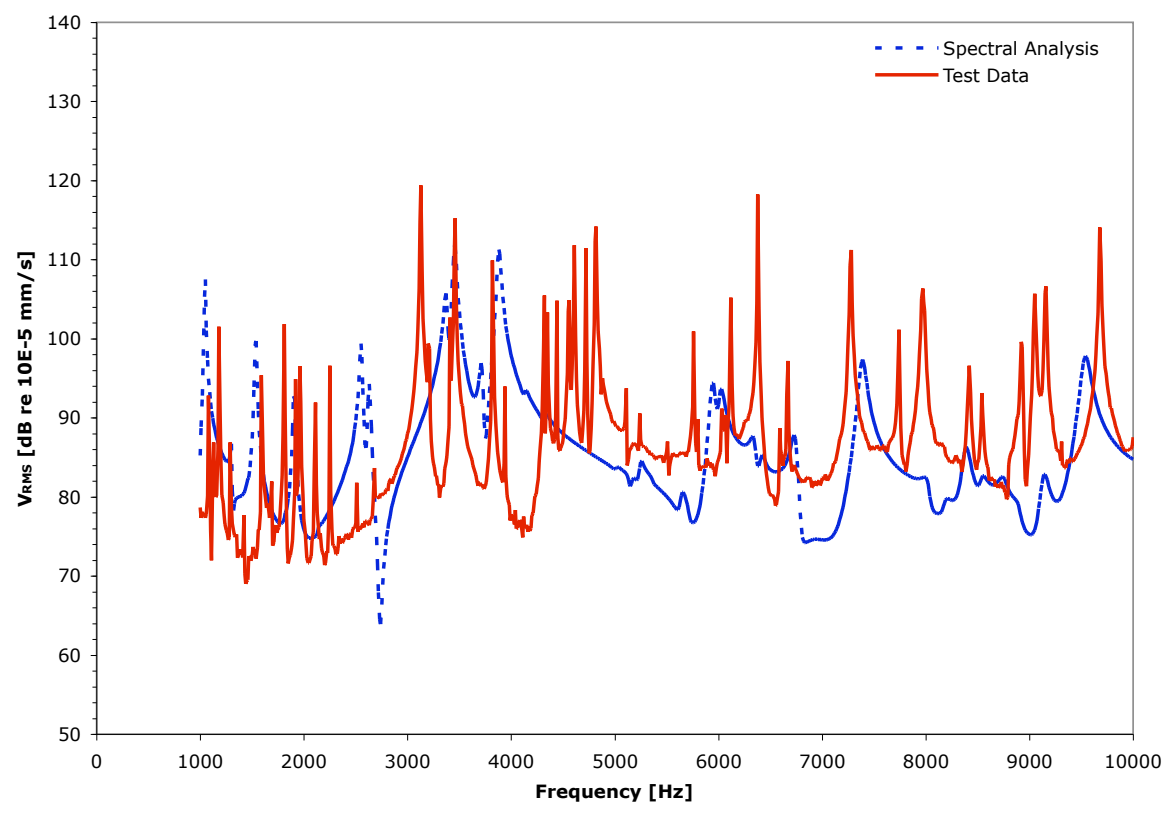

Figure 5.12: Spectral finite element vs. experimental RMS transverse velocity (computed using the response from 2 top layer nodes) for square core structure

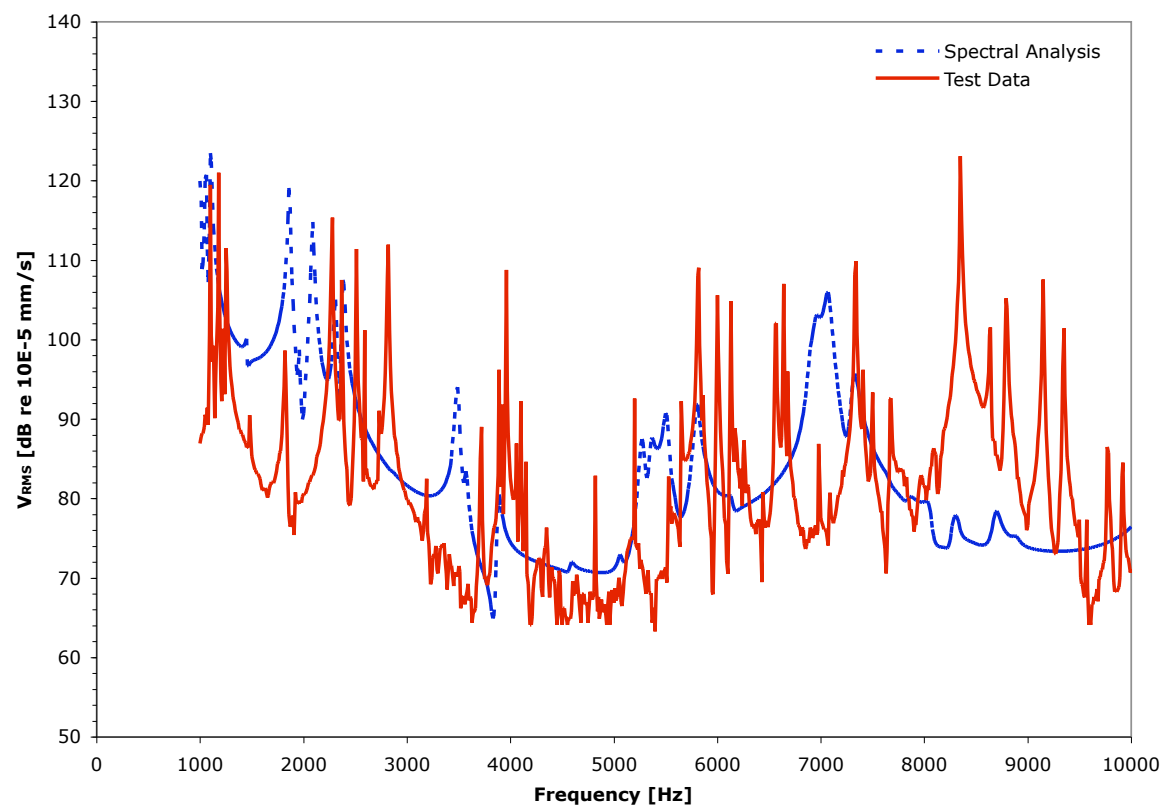

Figure 5.13: Spectral finite element vs. experimental RMS transverse velocity (computed using the response from 2 top layer nodes) for compliant mechanism structure 
$3 \mathrm{kHz}$. Additionally, the two peaks around $4 \mathrm{kHz}$ that dominate the computational response are split into two broader bands of resonant peaks spanning $3 \mathrm{kHz}$ to $5 \mathrm{kHz}$ in the experimental data. Above $5 \mathrm{kHz}$, response matches in an approximate sense, however the amplitude of resonance and the sharpness of each peak at $6380 \mathrm{~Hz}$ and $9680 \mathrm{~Hz}$ in the experimental data suggests lower levels of structural damping.

The compliant mechanism structure demonstrates similar behavior (Figure 5.13). The shift of measured response towards higher frequencies is more noticeable implying a structure that is substantially stiffer. Despite this, upon close examination, the underlying structural dynamic characteristics of interest are verified. Specifically, the attenuation zones in the experimental data for the compliant mechanism structure are present. However, the first attenuation zone has moved approximately 400 $\mathrm{Hz}$ higher and extends from $2700 \mathrm{~Hz}$ to $5700 \mathrm{~Hz}$. The large peak in the computed response at $7080 \mathrm{~Hz}$ has shifted to $8350 \mathrm{~Hz}$ in the experimental data. Following this, the reduction in the computed response at $8200 \mathrm{~Hz}$, which leads into the beginning of the second attenuation zone, is present in the experimental data from $9600 \mathrm{~Hz}$ to $10 \mathrm{kHz}$. Despite this shifting, within each measured attenuation zone for the compliant mechanism structure, significant reduction in response is evident relative to measured response levels for the square core structure.

\subsection{Experimental Validation}

As can be seen clearly in Figures 5.8 and 5.9, the interfaces between the members that comprise each structure are not ideal point connections as assumed using onedimensional spectral elements. Instead, these connections have a continuum nature and a non-zero fillet, which are consequences of the chosen manufacturing process. These radii act to stiffen response. Accordingly, to better capture this continuum 
characteristic, a conventional two-dimensional finite element analysis using continuum elements was performed.

\subsubsection{Analysis: Continuum Elements}

For the conventional finite element model the nodal loading equivalent to the localized incident pressure wave on a bottom layer element of the structure is found in a manner similar to that for the spectral method

$$
\mathbf{f}^{e}\left(\mathbf{w}_{b}, \omega\right)=\int_{0}^{L^{e}} \mathbf{w}_{b}(x, \omega) p_{\text {in }} d x
$$

where in this instance $\mathbf{w}_{b}$ is the conventional weighting function for a continuum shell element.

\subsubsection{Computational Results: Continuum Elements}

A Nastran Direct Frequency Response analysis using the SOL 108 solution sequence was performed from $0 \mathrm{~Hz}$ to $10 \mathrm{kHz}$ at $10 \mathrm{~Hz}$ frequency steps. The conventional square core benchmark structure was modeled using 11,556 CQUAD4 elements. A similar analysis was performed for the compliant mechanism structure using 17,152 elements. Bottom layer localized forcing of each structure was based on an equivalent nodal loading calculated from an excitation SPL of $145.4 \mathrm{~dB}$.

Figures 5.14 and 5.15 give the computational and experimental results for the square core structure and compliant mechanism structure, respectively. A structural damping parameter of 0.003 provides the best fit between the analysis and the mea-

sured data in terms of resonant peak amplitude. A preliminary visual inspection of these figures shows that the incidence of resonances, anti-resonances, and minima generally agree, thus substantiating the refined computational approach.

Accounting for slight shifts in resonant peaks, the agreement between the conventional finite element analyses and the test data is reasonable considering the breadth 


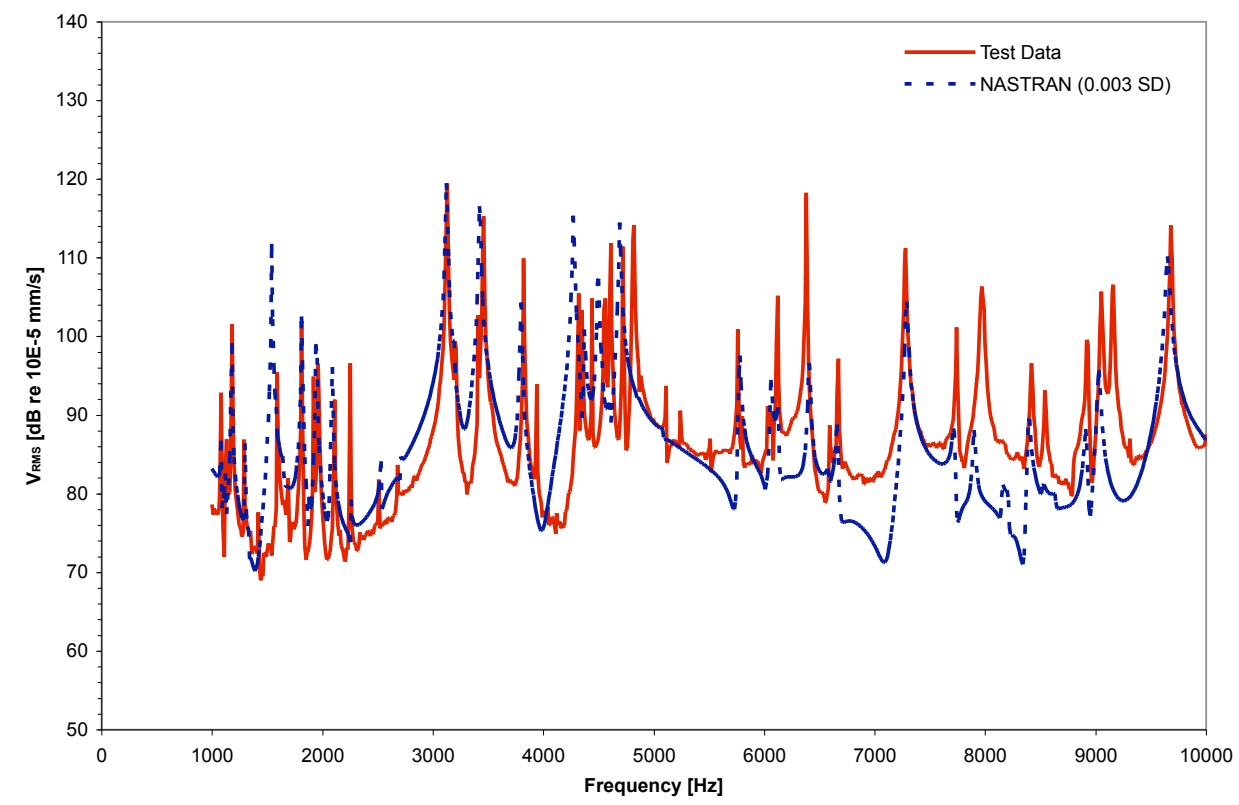

Figure 5.14: Nastran vs. experimental RMS transverse velocity (computed using the response from 2 top layer nodes) for square core structure

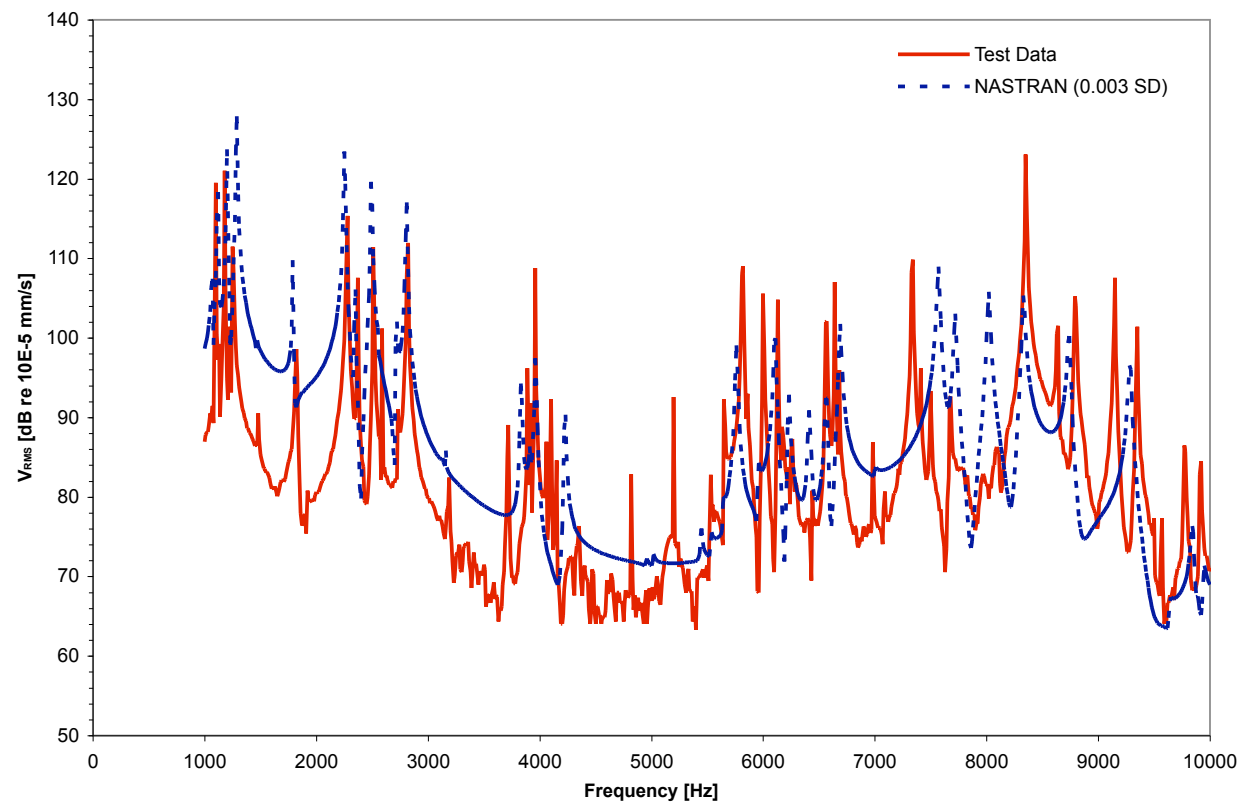

Figure 5.15: Nastran vs. experimental RMS transverse velocity (computed using the response from 2 top layer nodes) for compliant mechanism structure 


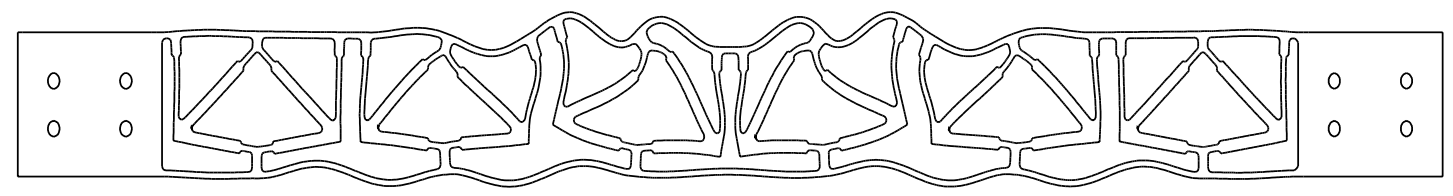

Figure 5.16: Global mode of compliant mechanism structure - $3963 \mathrm{~Hz}$

of the test frequency range. Attenuation is seen in the expected frequency ranges for the compliant mechanism structure. The average RMS transverse velocity from 3000 $\mathrm{Hz}$ to $5500 \mathrm{~Hz}$ is $88.9 \mathrm{~dB}$ for the square core structure and $72.1 \mathrm{~dB}$ for the compliant mechanism structure. This represents a $16.8 \mathrm{~dB}$ reduction in response within the first attenuation zone. Maximum attenuation upwards of $45 \mathrm{~dB}$ is seen at $3460 \mathrm{~Hz}$. The primary limiting factor for the first attenuation zone is the global mode at 3963 Hz shown in Figure 5.16, which is predicted by the analysis, yet due to lower levels of structural damping results in a larger response amplitude. From $9300 \mathrm{~Hz}$ to 10 $\mathrm{kHz}$ the average RMS transverse velocity is $89.9 \mathrm{~dB}$ for the square core structure. Over the same frequency range, the compliant mechanism structure has an average RMS transverse velocity of $75.1 \mathrm{~dB}$, which represents a reduction of $14.8 \mathrm{~dB}$ within the start of the second attenuation zone.

\subsection{Discussion}

The percent error between the measured and computed RMS transverse velocity responses for the two structures is given in Figures 5.17 and 5.18. Over the $1 \mathrm{kHz}$ to $10 \mathrm{kHz}$ frequency range the square core structure has a measured RMS transverse velocity average error of $6.2 \%$ and a more conservative root-mean-square error (RMSE) of $8.1 \%$ when compared with the Nastran predicted response (Fig- 


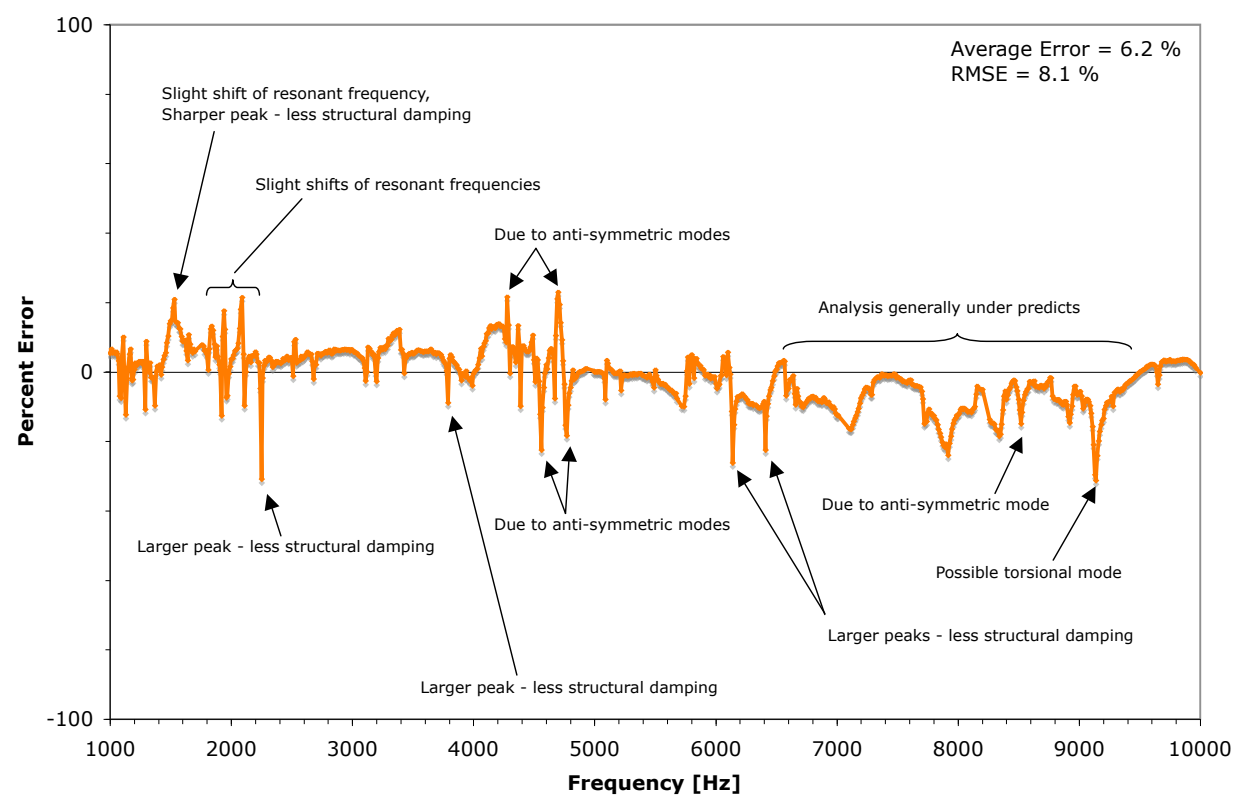

Figure 5.17: Percent error between Nastran and experimental RMS transverse velocity results for square core structure

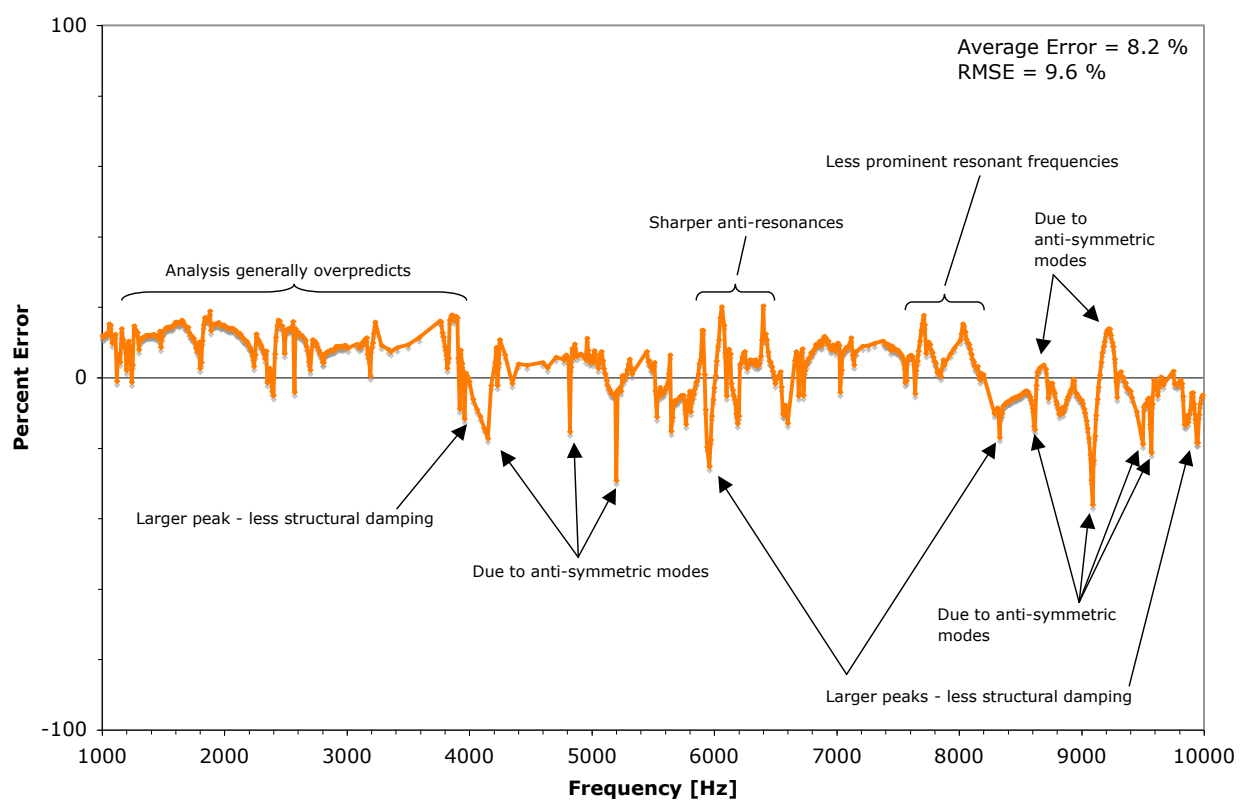

Figure 5.18: Percent error between Nastran and experimental RMS transverse velocity results for compliant mechanism structure 
ure 5.17). For the prototype compliant mechanism core structure the average error value increases slightly to $8.2 \%$ and the RMSE value increases to 9.6\% (Figure 5.18).

Likely sources of error are highlighted in these figures. There are slight (i.e. generally ranging from $10-70 \mathrm{~Hz}$ ) shifts of resonant frequencies relative to the computational analysis. In both analysis and experiment, a $10 \mathrm{~Hz}$ frequency step was employed, which allowed for relatively acceptable resolution given the very large breadth of the overall frequency range. Nonetheless, slight shifts in resonant peaks are likely attributable to the lack of fine scale fidelity.

The presence of anti-symmetric or double modes also is clear based on a Normal Modes analysis (SOL 103) performed for each structure. It is well known that perfectly symmetric, periodic structures become quasi-periodic once minor manufacturing tolerances and slight test setup misalignments are introduced, per Ewins [23] and Pierre, Castanier, \& Chen [80]. Disorder effects redistribute energy, exciting anti-symmetric or double modes that do not exist in a computational analysis where geometry, boundary conditions, and loading are assumed to be perfectly symmetric. Furthermore, in the case of the compliant mechanism structure, the acoustic excitation is applied at a location of relatively high flexibility. This results in possible local effects that, coupled with quasi-periodicity, excite anti-symmetric modes.

The close proximity of symmetric and anti-symmetric normal mode frequencies is seen in Figures 5.19 (i.e. $8739 \mathrm{~Hz}$ ) and 5.20 (i.e. $8797 \mathrm{~Hz}$ ), respectively. Each figure presents modal results from the normal modes analysis. In Figure 5.21 a zoomed view of the computational versus experimental RMS normal velocity response of the compliant mechanism structure is given from $8200 \mathrm{~Hz}$ to $10000 \mathrm{~Hz}$. The Nastran direct frequency response analysis also predicts the symmetric resonant peak at 8739 $\mathrm{Hz}$ highlighted in this figure, but not the anti-symmetric peak at $8797 \mathrm{~Hz}$. However, 


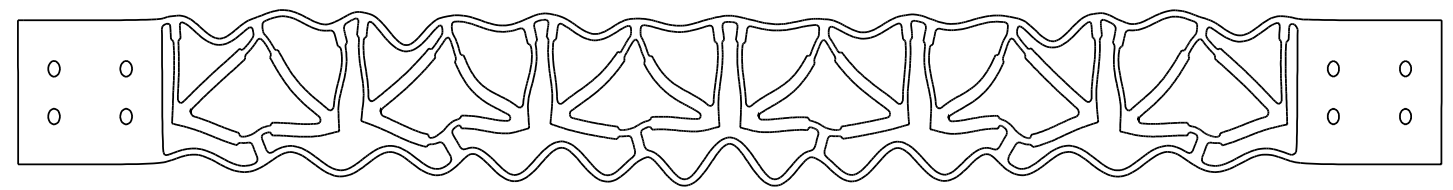

Figure 5.19: Symmetric mode of compliant mechanism structure $-8739 \mathrm{~Hz}$

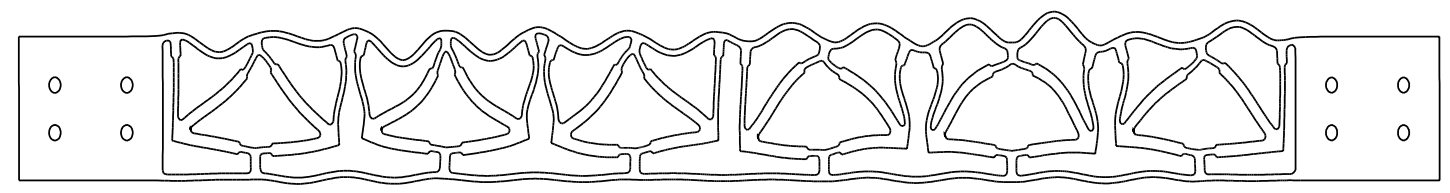

Figure 5.20: Anti-symmetric mode of compliant mechanism structure $-8797 \mathrm{~Hz}$

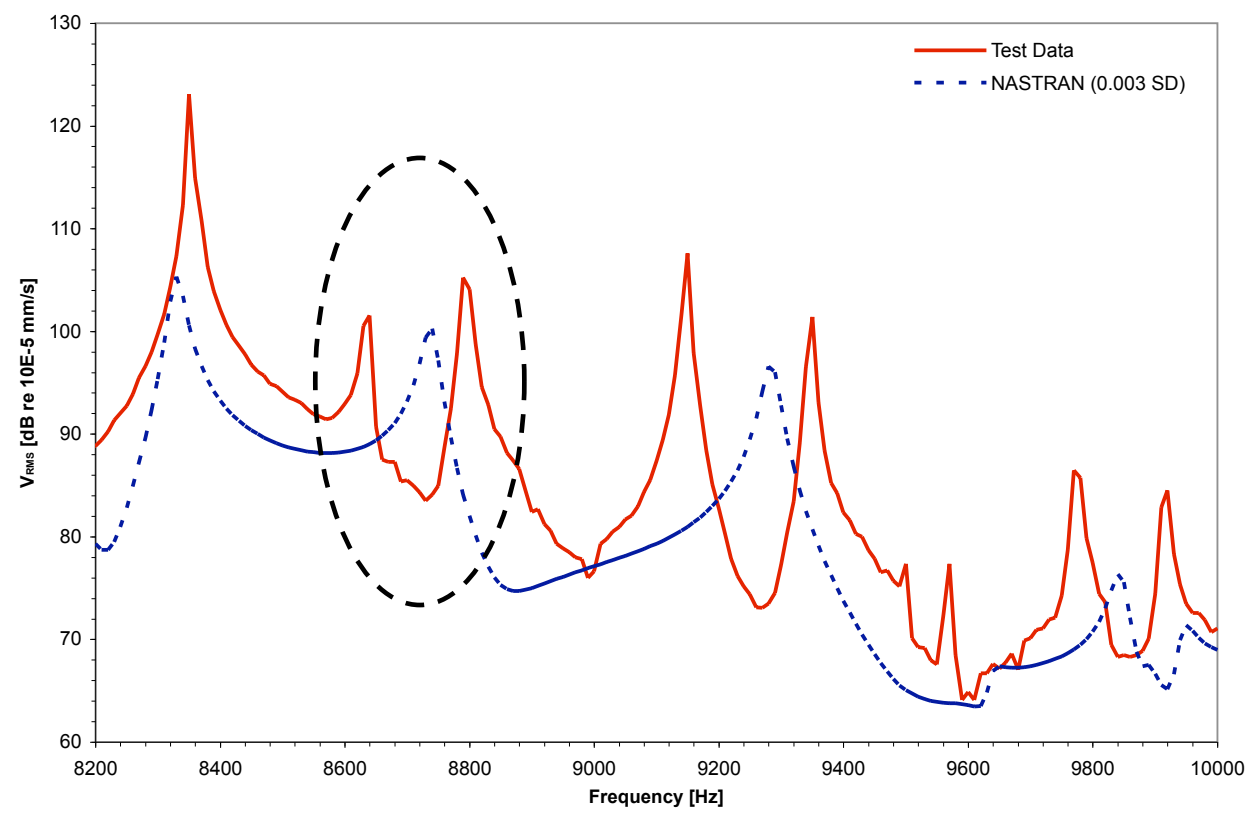

Figure 5.21: Nastran vs. experimental RMS transverse velocity (computed using the response from 2 top layer nodes) for compliant mechanism structure. Region of interest is highlighted by the dashed oval. 
the experimental data in this figure shows the separation of the symmetric mode into two modes occurring at $8650 \mathrm{~Hz}$ and $8790 \mathrm{~Hz}$. Thus, the experimental data confirms the presence of two modes very close to each other in this frequency range and splitting the computed peak into two. This final source of error is visible at several other locations (e.g. at $9290 \mathrm{~Hz}$ in Figure 5.21) throughout the $1-10 \mathrm{kHz}$ frequency range for each structure and accounts for several outlying values.

Despite the above sources of error, the enhanced vibratory characteristics of the compliant mechanism structure are verified using the refined computational approach, along with the experimental data presented in this section. These results suggest a two stage design process. In the first stage, the one-dimensional spectral method is employed to design the basic compliant mechanism unit cell. A subsequent, two-dimensional analysis should then be completed, utilizing the manufactured mechanism design to identify the most significant variations from the idealized one-dimensional analytical prototype. 


\section{CHAPTER 6}

\section{Methodology \& Applications}

A hierarchical approach has been implemented in the design of structures with integral compliant mechanisms. Generally, local units cells have been utilized as building blocks for the design of global structures. Specifically, unit cell size and material constraints have been addressed. The connectivity of unit cells in parallel and serial fashion has also been investigated, and these investigations have led to different functional results. Extensions to three-dimensional design were proposed. Furthermore, numerical studies on unit cell size and topology optimization were presented to demonstrate that different unit cell design approaches exist. This computational work was then followed by experimental studies that were focused on the verification (i.e. "proof-of-concept") of the fundamental hypotheses of this research.

Given this, the main objective of this chapter is to distill the various results from these studies into an overarching design methodology. The methodology is focused on providing practical guidance to a structural analyst in the field when designing structures with integral compliant mechanisms.

An overview of this methodology is given in Section 6.1. Novel applications of the methodology are then discussed in Section 6.2. 


\subsection{Methodology Overview}

The following is a list of the minimum steps necessary for designing structures with integral compliant mechanisms for mid-frequency response:

1. Define the size, weight, and material constraints for the global structure of interest along with the frequency range over which attenuation is sought.

2. Define the initial unit cell size based on the information from Step 1 and an initial wavenumber-frequency analysis, reference Eqn (3.16).

3. Synthesize a compliant mechanism unit cell topology using either a priori knowledge, or a genetic algorithm topology optimization routine.

4. Compute the unit cell top layer RMS normal velocity response, FRF, and the deformed shape/total energy distribution in order to characteristic basic structural dynamic response.

5. If necessary, "tune" the basic structural dynamic response of the unit cell over the frequency range of interest using a genetic algorithm size optimization routine.

6. Determine if enhanced vibro-acoustic response is sought. If so, continue to Step 7. Otherwise, integrate the unit cell into the global structure, compute the RMS normal velocity response of the structure, compare the structural dynamic response with requirements, and proceed to Step 10.

The prior steps are those required for realizing simple truss-like beam structures. If greater vibro-acoustic attenuation or a multi-functional lattice structure is desired, the additional steps to follow are: 
7. For structures that are subjected to local forcing - Examine a global configuration where compliant mechanism unit cells are attached in a parallel fashion to the remainder of the global structure. The compliant mechanism unit cells should be integrated into the structure in the region of the local forcing as done in Section 3.4.1. Compute the RMS normal velocity response of the structure, compare the structural dynamic response with requirements, and proceed to Step 10.

8. For structures exhibiting greater vibro-acoustic attenuation - Examine the serial attachment of additional compliant mechanism layers to increase the unit cell height as done in Sections 3.4.2 and 3.4.3. Compute the RMS normal velocity response of the serial unit cell, integrate the unit cell into the global structure, compute the RMS normal velocity response of the structure, compare the structural dynamic response with requirements, and proceed to Step 10.

9. For structures exhibiting multi-functional properties - Examine the serial attachment of a compliant mechanism unit cell layer to an existing structure as done in Section 3.4.4. Compute the RMS normal velocity response of the multifunctional unit cell, integrate the unit cell into a global structure, compute the RMS normal velocity response of the structure, compare the structural dynamic response with requirements, and proceed to Step 10.

10. Verification of the dynamic response of a prototype structure with integral compliant mechanisms - As a final stage in the overall design process, complete a continuum computational analysis utilizing the manufactured compliant mechanism structure design to identify the most significant variations from the idealized spectral element model. 
The above steps are "general guidelines" to be followed in the design of structures with integral compliant mechanisms for mid-frequency response. They are also iterative and depend on how well the computed structural dynamic response fulfills requirements. Accordingly, Step 3 and the subsequent process should be revisited as necessary. The following applications exercise portions of the above methodology for select 2-D and 3-D problems of interest.

\subsection{Applications}

Two applications of the above methodology, plus underlying motivation, are examined in this section. The first application is a basic 2-D conceptual study on the design of acoustic isolation panels for the reduction of structural vibrations generated by turbulent boundary layer flow. The second application explores the design of a torsional coupler for the reduction of the axial vibrations transmitted between two joined and rotating circular shafts. This application addresses the modeling of a more complicated three-dimensional circular geometry layout.

\subsubsection{Acoustic Isolation Panels}

As discussed in the motivation section of this dissertation (Section 1.1), turbulent boundary layer flow across the external surface of an aircraft produces structural vibrations, which cause noise within the aircraft cabin. The frequency spectrum of turbulent boundary excitation for a typical commercial passenger aircraft extends upwards of $10 \mathrm{kHz}$, as shown in Figure 6.1, and as discussed by Bhat \& Wilby [5, 6]. Figure 6.2 illustrates the use of damping tape and/or sponge rubber wedges as a common method of reducing internal cabin noise. Alternatively, this investigation proposes a passive multi-functional structure that serves a structural function and an acoustic function in the attenuation of this internal cabin noise. 


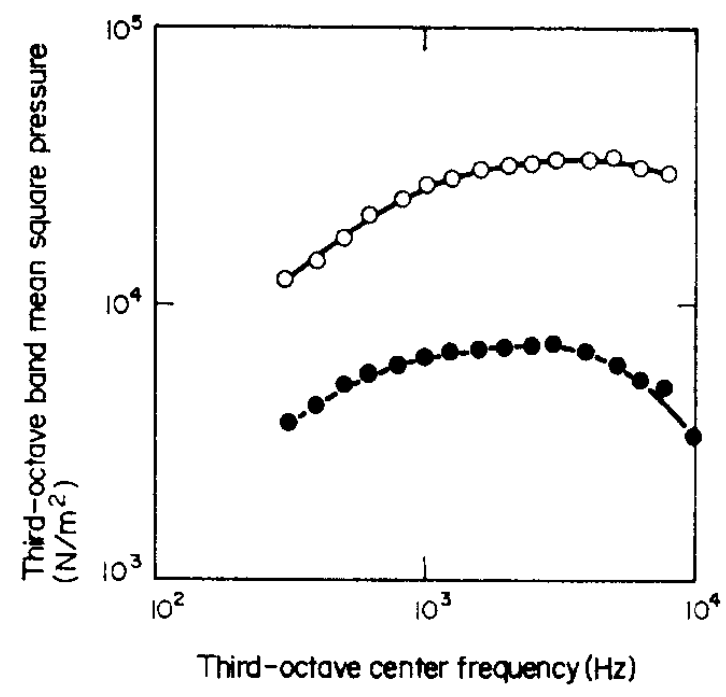

Figure 6.1: Estimated pressure spectrum for turbulent boundary layer excitation: Boeing model 727-200 aircraft (upper curve for Mach 0.85, lower curve for Mach 0.55), from Bhat \& Wilby [6]

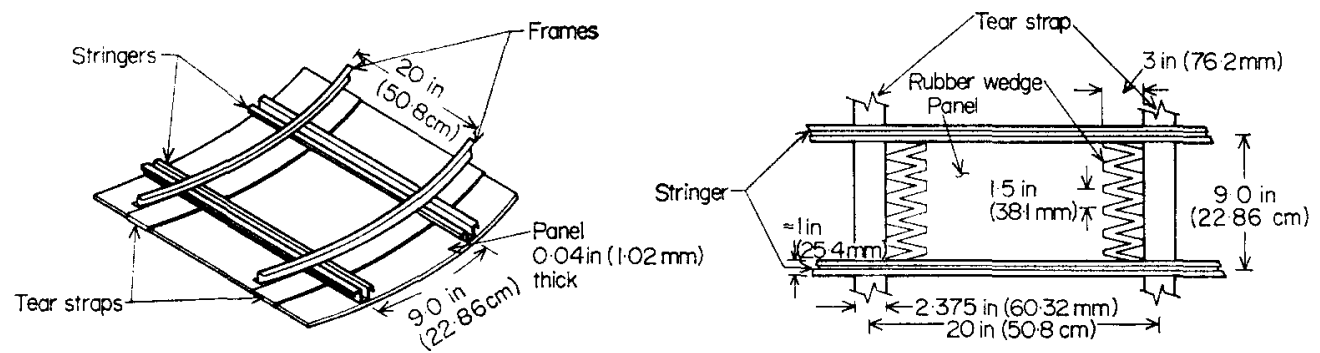

Figure 6.2: Representation of aircraft fuselage structure, from Bhat \& Wilby [6] 


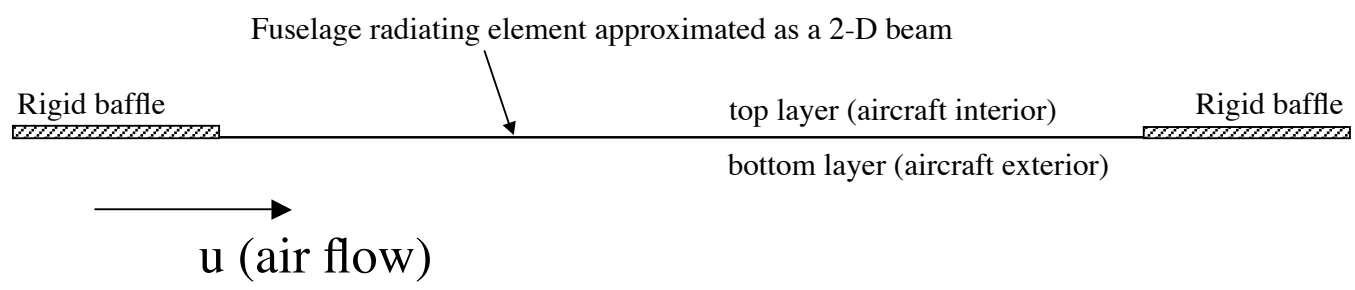

Figure 6.3: Conceptual model of fuselage radiating element approximated by a twodimensional beam

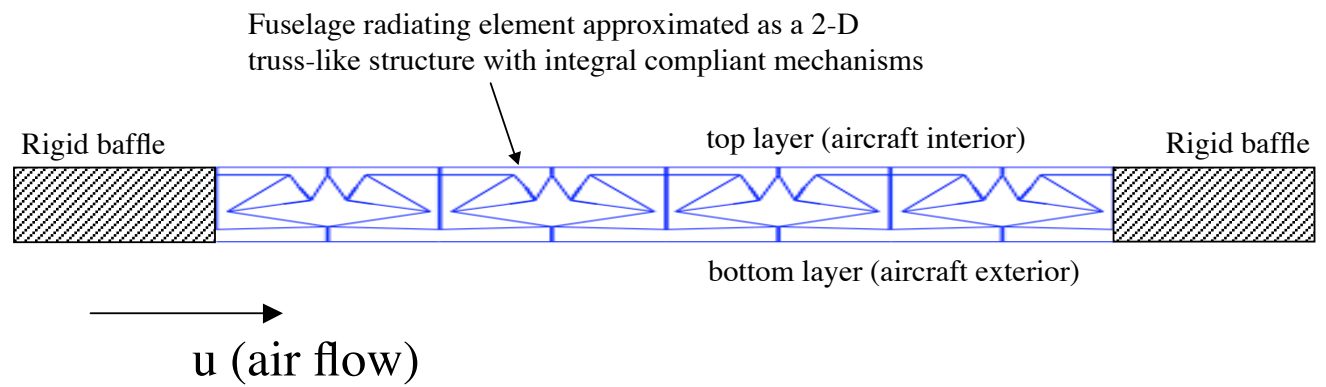

Figure 6.4: Conceptual model of fuselage radiating element approximated by a twodimensional truss-like structure with integral compliant mechanisms

According to Bhat \& Wilby $[5,6]$, a typical radiating element of the fuselage structure is defined by the amount of fuselage skin contained with the boundary defined by the frames and stringers (Figure 6.2). Thus, the structural dynamic response of this radiating element is the subject of interest for this study. Two simplified two-dimensional conceptual models of this radiating panel element are shown in Figures 6.3 and 6.4. Both models are considered to be 2-D edgewise beam approximations of the 3-D radiating panel element from Figure 6.2. Since airflow over the exterior of an aircraft tends to be unidirectional (i.e. along the length of the fuselage), this is considered a reasonable assumption. In the first model (Figure 6.3), the structural dynamic response of a simple beam, representing the bare fuselage 
skin, is examined. The top side of the structure represents the aircraft interior and the bottom side represents the exterior of the aircraft, which is subject to a turbulent boundary layer flow. In the second model (Figure 6.4), a truss-like structure with integral complaint mechanisms is shown. The top layer again represents the aircraft interior, which is set apart from the bottom layer by a compliant mechanism core. The bottom layer is assumed to represent the exterior fuselage skin that is subjected to a turbulent boundary layer flow.

The design of the structure shown in Figure 6.4 was carried out using the methodology summarized in Section 6.1. While material and structural size constraints were defined, weight constraints were not established since the determination of the general feasibility of the concept was the objective of this study. Despite this, weight comparisons are made since added mass is a crucial metric in any aerospace application.

The material for both structures was defined as aluminum (i.e. $E=7.1 \times 10^{10}$ $\mathrm{N} / \mathrm{m}^{2}, \rho=2700 \mathrm{~kg} / \mathrm{m}^{3}$, and $\eta=0.01$ ) based on the knowledge that the fuselage skin of most commercial aircraft is typically made of aluminum or a similar lightweight material, per Bhat \& Wilby $[5,6]$. The primary size constraint for the compliant mechanism structure is the in-plane thickness since it determines the amount by which the structure extends into the cabin interior. The in-plane thickness of the compliant mechanism structure was set at $0.05 \mathrm{~m}$. This thickness was deemed adequate since it is less than the thickness of typical sponge rubber wedges (i.e. $\sim 0.08$ $\mathrm{m}$ thick per Bhat \& Wilby $[5,6])$ that are mounted to the interior of the fuselage skin, reference Figure 6.2. Secondary size constraints are imposed by the overall size of a typical fuselage radiating element. Based on the dimensions in Figure 6.2 the in-plane length (i.e. the length parallel to the air flow direction) for the two models 
was set to $0.4 \mathrm{~m}$. The bare fuselage skin was assigned a thickness of $1.1 \mathrm{~mm}$, which is also the width assigned to the bottom layer of the compliant mechanism model. The out-of-plane thickness for both models was set to $0.2 \mathrm{~m}$.

Given these material and overall size constraints, the next step was to define the frequency range of interest for the study and the local unit cell size based on an initial wavenumber-frequency analysis. The pressure spectrum at a subsonic speed of Mach 0.85 (Figure 6.1) was used to define the frequency range. This spectrum spans a frequency range from approximately $350 \mathrm{~Hz}$ to $8500 \mathrm{~Hz}$. Observe that the largest excitation amplitudes occur at frequencies above $1 \mathrm{kHz}$. Thus, the goal in sizing the bottom layer of the compliant mechanism unit cell was to place both the first and second attenuation zones of the unit cell within a $1000 \mathrm{~Hz}$ to $8500 \mathrm{~Hz}$ frequency range. Appropriately, this maximizes the breadth of both attenuation zones inside the frequency range of interest.

Using a unit cell bottom layer thickness of $1.1 \mathrm{~mm}$ and a unit cell length of $0.1 \mathrm{~m}$ sets the attenuation zone starting frequencies at $1020 \mathrm{~Hz}$ and $4060 \mathrm{~Hz}$, per Eqn (3.16). Both of these frequencies are located above $1000 \mathrm{~Hz}$ and below 8500 $\mathrm{Hz}$, thus increasing the amount of attenuation occurring inside the sought frequency range.

The topology used in the design of the compliant mechanism structure was determined in an a priori fashion based on the work in Section 3.2.1. The topology in Figure 6.4 is identical to the one first shown in Figure 3.9. The core members of the unit cell were assigned the same thickness as the bottom layer (i.e. $1.1 \mathrm{~mm}$ ). The transmission and ground members of the unit cell were assigned a $50 \%$ larger width of $1.65 \mathrm{~mm}$.

The analysis of the compliant mechanism unit cell, bare fuselage structure, and 


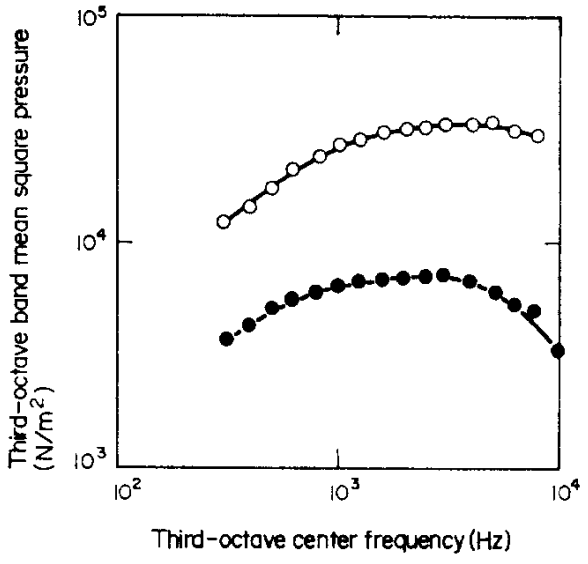

(a) Original data

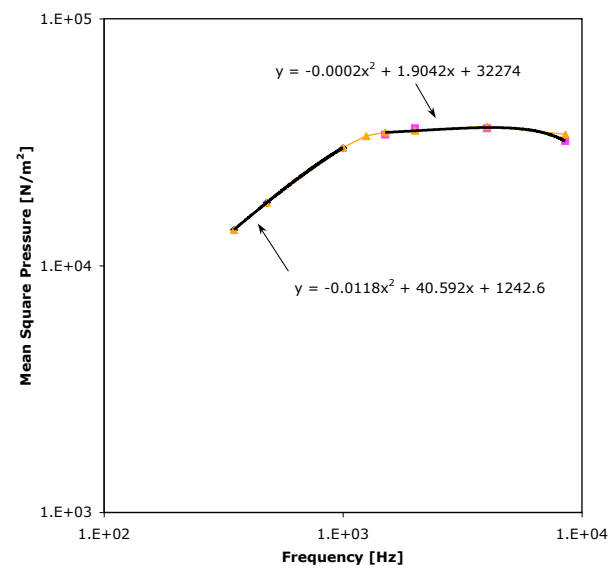

(b) Reconstructed data

Figure 6.5: Estimated pressure spectrum for turbulent boundary layer excitation: (a) Boeing model 727-200 aircraft (upper curve for Mach 0.85, lower curve for Mach 0.55), from Bhat \& Wilby [6]; (b) reconstructed data

compliant mechanism structure was executed using the spectral finite element method. The frequency dependent forcing function for each computational analysis was determined by manually reconstructing the Mach 0.85 pressure spectrum data from Figure 6.1. The curve fitted data is compared with the original data in Figure 6.5. The top layer RMS normal velocity of each structure was computed from $350 \mathrm{~Hz}$ to $8500 \mathrm{~Hz}$ evaluated in $10 \mathrm{~Hz}$ frequency steps. The top layer RMS transverse velocity response of the unit cell, bare fuselage structure, and compliant mechanism structure is shown in Figure 6.6.

This figure clearly shows the location of both the first and second attenuation zones, which lay fully within the desired frequency range. Below $1 \mathrm{kHz}$, the dynamic response of the bare fuselage structure and compliant mechanism structure match in an approximate sense signifying the dominance of global level response. Above $1 \mathrm{kHz}$, the wavenumber-frequency analysis correctly predicts the beginning of both attenuation zones for the compliant mechanism structure at $1020 \mathrm{~Hz}$ and $4060 \mathrm{~Hz}$. 


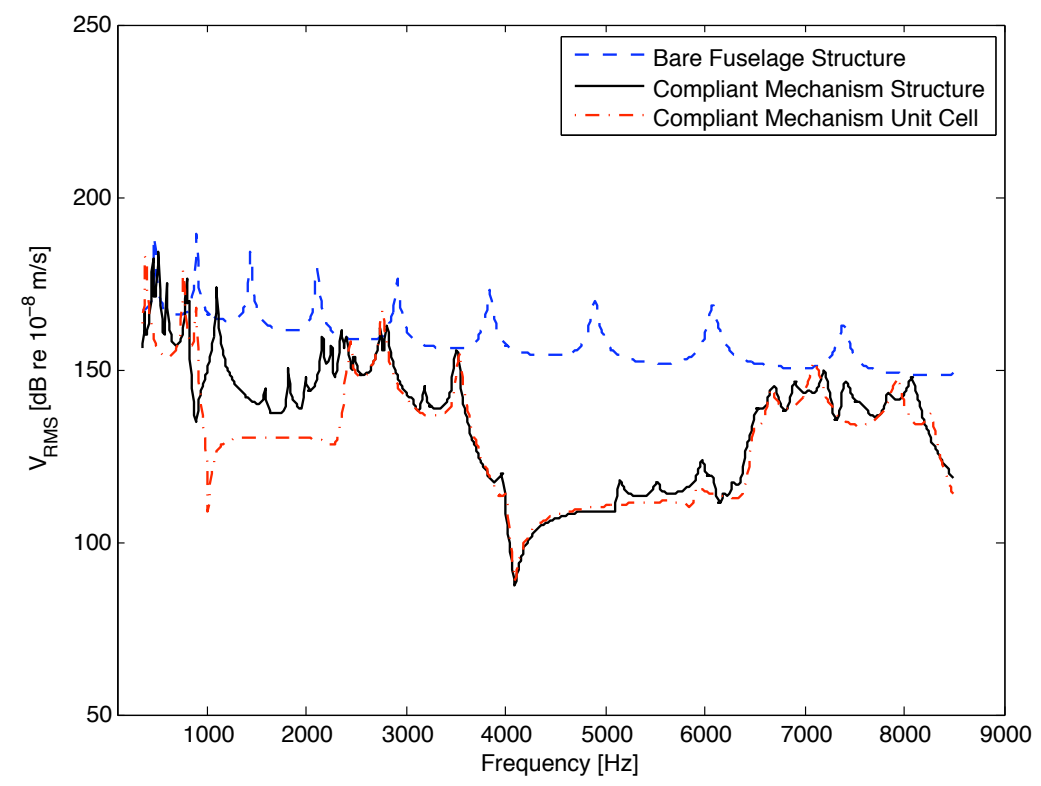

Figure 6.6: Top layer RMS velocity of bare fuselage structure, compliant mechanism structure, and compliant mechanism unit cell

The FRF and deformed shape/total energy distribution analyses have been foregone since they were adequately covered in a prior chapter for this compliant mechanism topology. Nonetheless, the expected reduction in response over the frequency range of $350 \mathrm{~Hz}$ to $8500 \mathrm{~Hz}$ is present with an average top layer RMS normal velocity that is approximately $24 \mathrm{~dB}$ lower than that of the bare fuselage skin. Even at resonant frequencies of the compliant mechanism structure, between 2200-3600 Hz and above $6500 \mathrm{~Hz}$, response levels are still equal to, or slightly below, those of the bare fuselage skin. This represents a significant decrease in structural dynamic response.

Exclusive of these improvements in response, the added mass of the compliant mechanism structure is not negligible. The mass of the compliant mechanism panel element is $2.03 \mathrm{~kg}$. Comparing this with $0.241 \mathrm{~kg}$, the mass of the bare fuselage panel element, makes clear a roughly one order of magnitude increase in weight. Despite this, there is fundamental difference between the two structures. Visual inspection of 


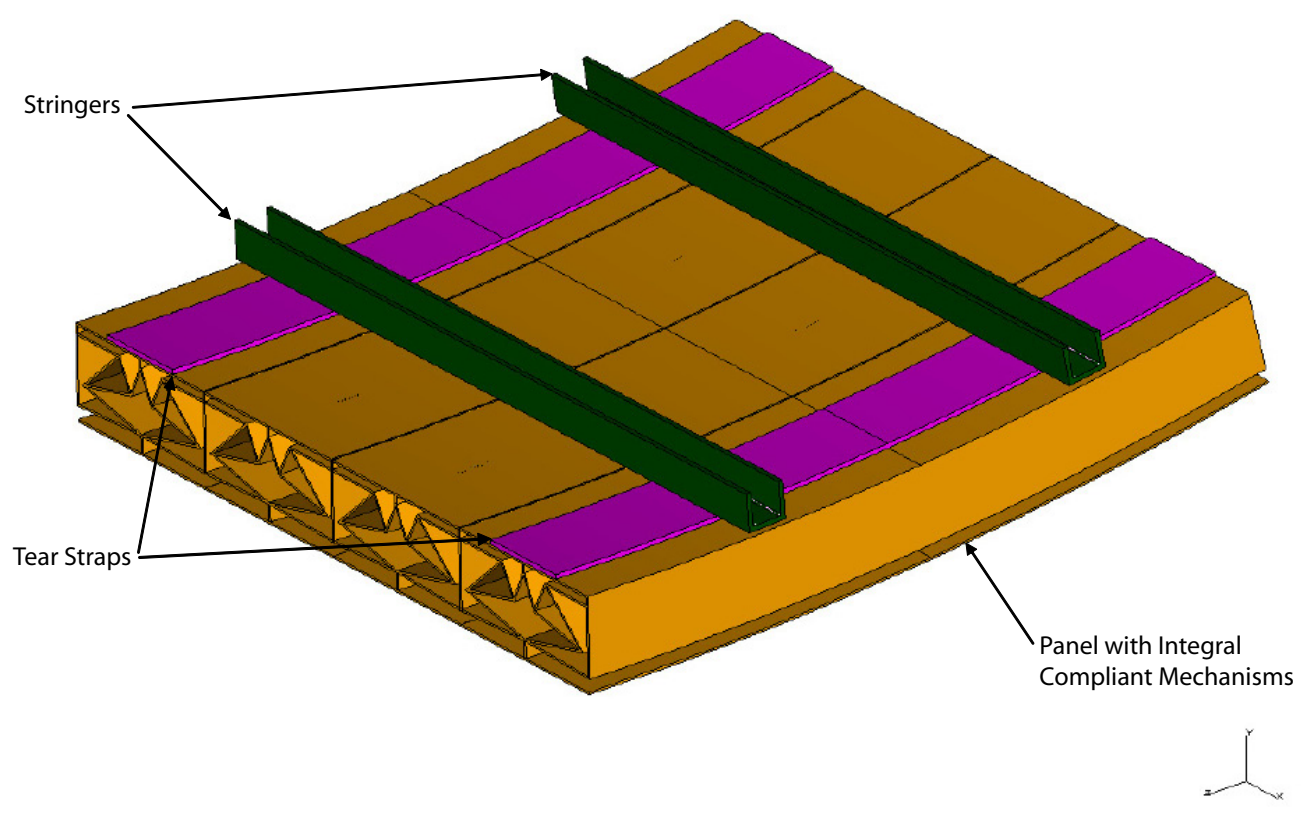

Figure 6.7: Conceptual model of fuselage structure with integral compliant mechanisms

the bare fuselage panel reveals that it must have a lower out-of-plane bending stiffness when compared with the compliant mechanism panel. Observe that the out-of-plane extrusion of the compliant mechanism structure generates an I-beam type structure. Thus, it is suggested that the frames shown in Figure 6.2 be either reduced in size or eliminated altogether. The compliant mechanism structure would then serve a multi-fuunctional role in reducing transmitted structural-borne noise while bearing load. Similarly, the stringers, while likely not eliminated, may potentially be reduced in size. A detailed design and analysis must be performed to fully compare mass and stiffness tradeoffs. Nonetheless, Figure 6.7 provides a conceptual model of what the potential structure might look like.

The concept presented in Figure 6.7 is but one embodiment of the ideas introduced in this dissertation. For this specific application, other design variations may 
be preferable from a weight perspective. For example, the fuselage structure could be manufactured out of a composite material to provide a better Young's modulus to density (i.e. strength to weight) ratio in Eqn (3.16); or, the fuselage structure could be left unmodified and injection molded acoustic lining panels could be attached to the interior of the structure instead. Furthermore, this application is one of many possible uses for this methodology. The design of "quiet" pipe walls for internal turbulent flows, and jackets of underwater sonar detention arrays for external turbulent flows, are other possible uses for this technology.

\subsubsection{Vibration Isolation Couplers}

Vibration transmission between two adjoined rotating shafts is a common problem encountered in many engineering applications including, but not limited to, automobiles, helicopters, ship propellers, and drilling machines. In these applications a motor is typically connected to a transmission from which a drive shaft extends. This drive shaft is usually attached to a subsequent pinion shaft by way of a universal joint. Typically, the pinion shaft is subject to vibrations induced by some excitation source (e.g. final drive gearing, a rotating propeller, or a drilling process). These vibrations are known to work their way back through the universal joint, drive shaft, and transmission resulting in excitation of resonant modes of the supporting structure or vehicle chassis. Common solutions consist of a vibration isolating coupler near the pinion shaft/universal joint/drive shaft assembly that limits vibration transmission transfered back to the structure. Many such solutions to this problem have been proposed in the patent literature as demonstrated by Johnson et al. [48], LoBosco et al. [60], Olson [76], Paulsen [78], and Takahashi et al. [96]. These solutions employ a resilient component that reduces the vibrations transmitted through the coupling. Thus, the purpose of this study is to systematically design a novel 
integral compliant mechanism structure, internal to a shaft coupler, to replace the traditional resilient component. The reduction of axial vibrations between coupled and rotating shafts in an automotive application is the primary focus of this study.

Interior noise in an automotive vehicle is a complex phenomenon that originates from many sources including the engine, suspension/chassis, brakes, drive-line dynamics, road noise, and traffic noise. These various sources generate sound inside the passenger cabin, and this sound spans the majority of the audible spectrum (e.g. $0 \mathrm{~Hz}$ to $14 \mathrm{kHz}$ ) per Bettella et al. [3], Douville et al. [16], Elliott [21], Ishiyama \& Hasimoto [45], and Oh et al. [75]. From this broad frequency range a reduced frequency range of interest was selected spanning $0-1 \mathrm{kHz}$. An attenuation zone starting frequency of $375 \mathrm{~Hz}$ was also selected since it is low enough to represent vibratory excitation originating from the vehicle drive-line dynamics or road noise.

A conceptual model of a vibration isolation coupler with an integral annular compliant mechanism structure is shown in Figure 6.8. The dimensions of the coupler were chosen arbitrarily in absence of a given set of design size requirements. Additionally, weight was not considered as a critical design constraint.

The conceptual model consists of a pinion shaft that is attached to a driven shaft via a coupler that allows the transmission of torque across the coupling, using a slip fit spline, while isolating axial vibrations. Vibration isolation is achieved by joining the two shafts with an annular compliant mechanism structure. In this manner, axial vibrations induced in the driven shaft by an excitation source (e.g. final drive gearing) are not transmitted back to the pinion shaft. In the event of failure of the compliant mechanism structure the cap component assures that the shafts do not decouple.

Two different materials were used in the design of the annular compliant mech- 


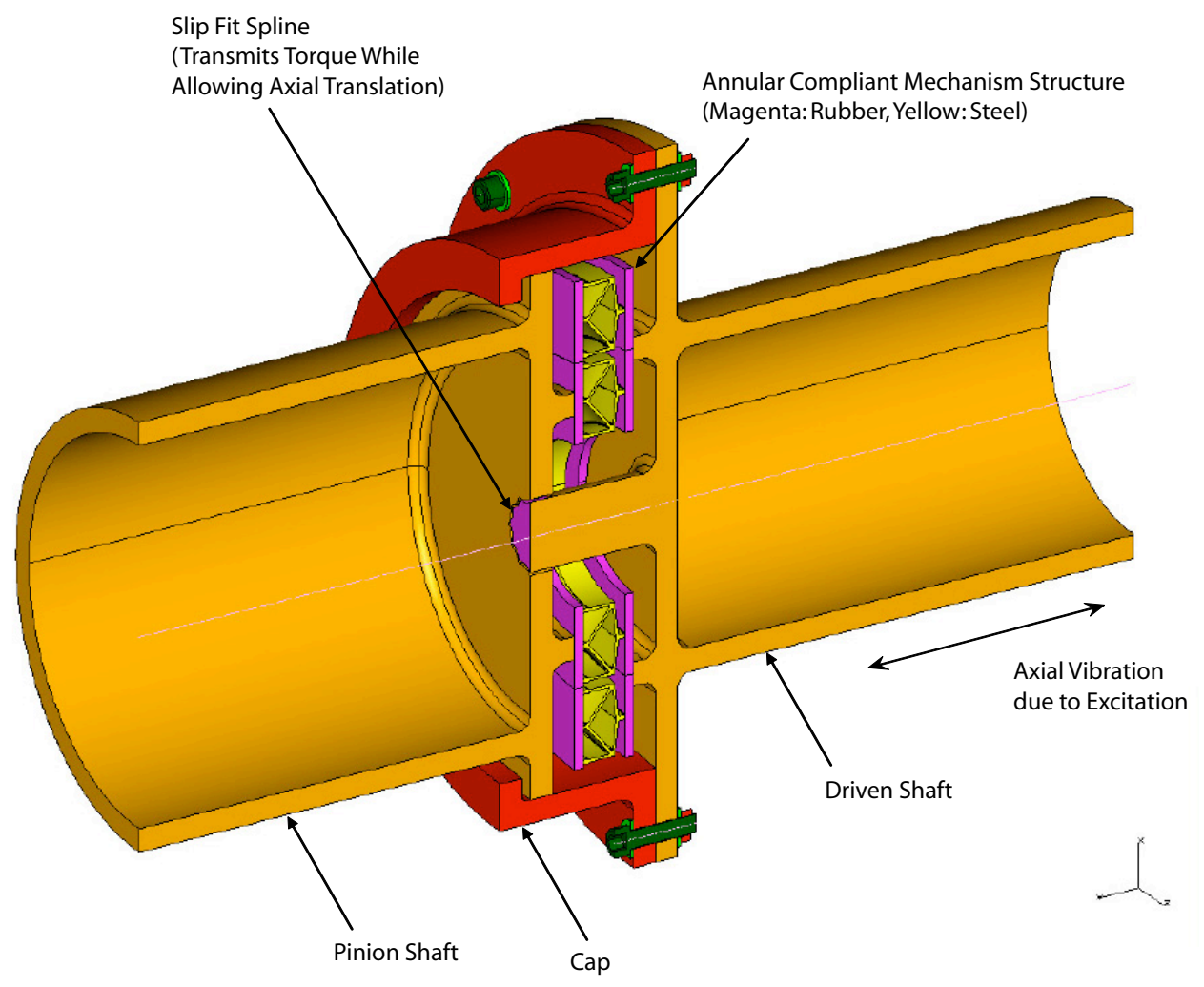

Figure 6.8: Conceptual model of vibration isolator coupling with an integral annular compliant mechanism structure

anism unit cell and structure. Specifically, vulcanized rubber and spring steel were used with the following material properties. For the vulcanized rubber, $E=1.5$ $\times 10^{6} \mathrm{~N} / \mathrm{m}^{2}, \rho=950 \mathrm{~kg} / \mathrm{m}^{3}$, and $\nu=0.48$. The use of a Poisson's ratio of 0.48 for the rubber material, instead of 0.5 , is necessitated by the assumption of linear response. For the spring steel, $E=21.0 \times 10^{10} \mathrm{~N} / \mathrm{m}^{2}, \rho=7850 \mathrm{~kg} / \mathrm{m}^{3}$, and $\nu=0.33$.

The rhombus compliant mechanism topology from Section 3.2.4 was selected for use in this application (reference Figure 3.27). Each top and bottom layer facesheet of the annular complaint mechanism structure was assigned the rubber material and a thickness of $2.5 \mathrm{~mm}$. The compliant mechanism members were assigned the 


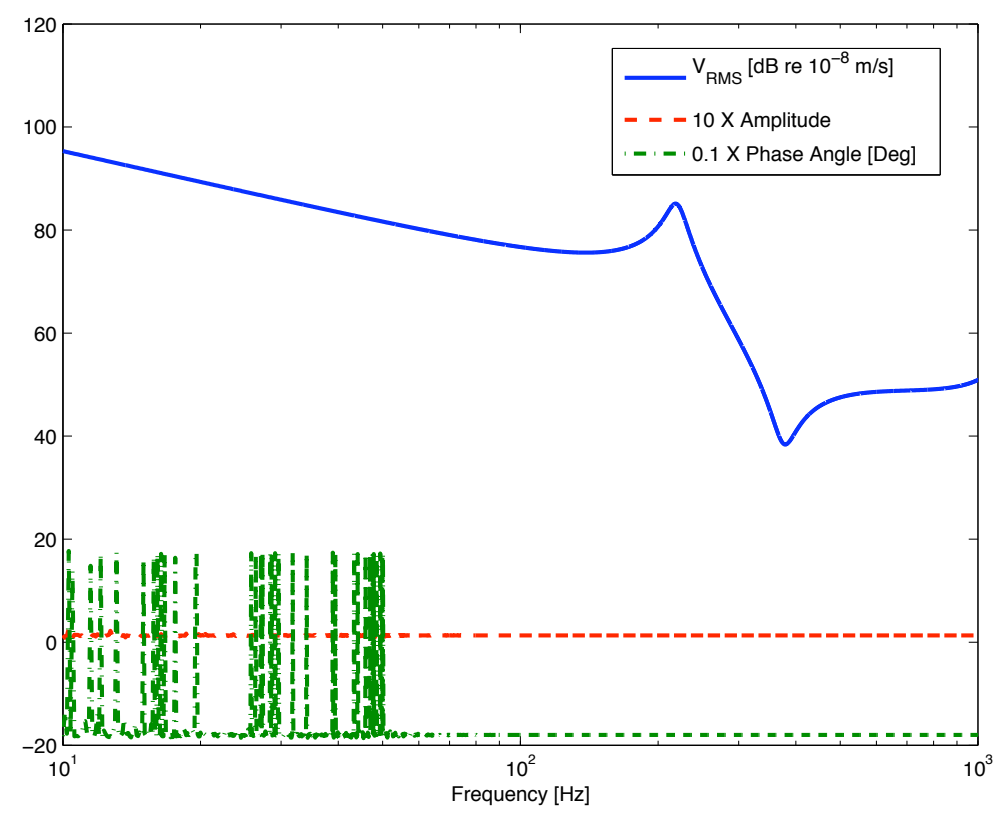

Figure 6.9: Top layer RMS velocity of multi-material rhombus compliant mechanism unit cell

steel material with all core members having a thickness of $0.762 \mathrm{~mm}$ and ground and transmission members having a $1.1 \mathrm{~mm}$ width. Thus, based on the specified bottom layer material (i.e. vulcanized rubber), facesheet thickness (i.e. $2.5 \mathrm{~mm}$ ), and preselected attenuation zone starting frequency (i.e. $375 \mathrm{~Hz}$ ) the length of the unit cell was determined using Eqn (3.16) as $21.9 \mathrm{~mm}$. Assuming two unit cells in parallel extending radially outward, the inner radius of the annular structure is 21.9 $\mathrm{mm}$ while the outer radius is $65.7 \mathrm{~mm}$. Thus, the overall diameter of the coupler assembly is $15.24 \mathrm{~cm}$ and the thickness is $5.08 \mathrm{~cm}$.

A two-dimensional unit cell analysis was executed using the linear spectral finite element method. The 2-D nature of the unit cell is a simplified representation of the full three-dimensional annular structure. Shown in Figure 6.9 is the RMS normal velocity response and FRF of the unit cell computed from $0 \mathrm{~Hz}$ to $1 \mathrm{kHz}$ at $0.1 \mathrm{~Hz}$ frequency intervals. The first attenuation zone clearly starts at $375 \mathrm{~Hz}$ and extends 


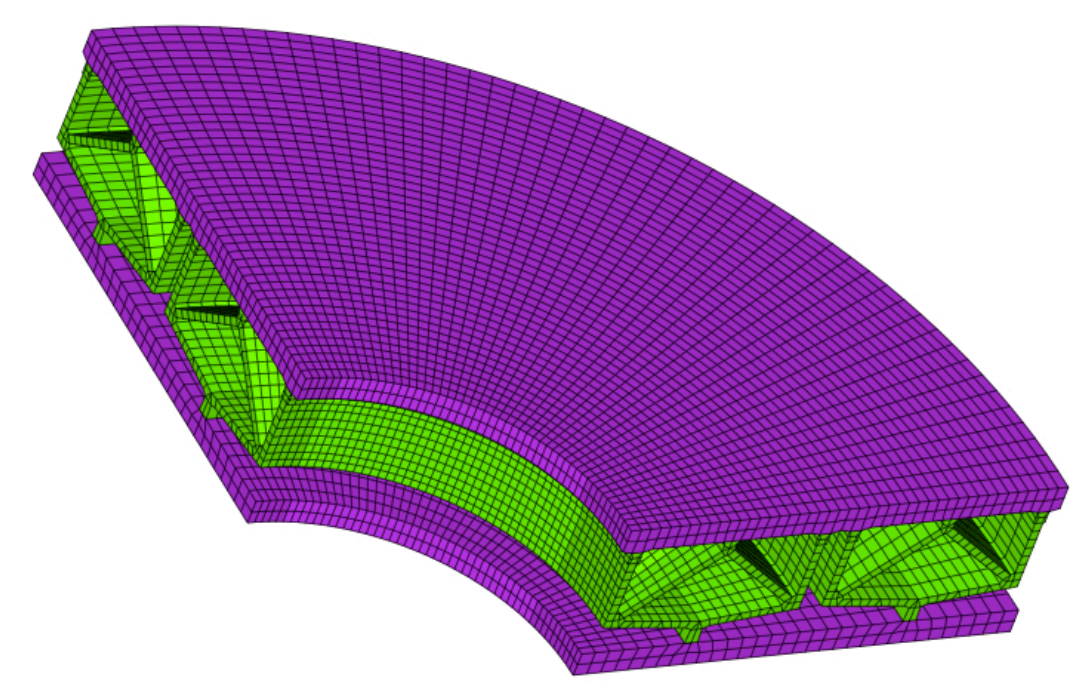

Figure 6.10: Finite element solid model of one quarter of the annular compliant mechanism structure (purple elements represent vulcanized rubber; green elements represent spring steel)

through $1 \mathrm{kHz}$. Given the unit cell sizing, the starting frequency for the second attenuation zone is $1410 \mathrm{~Hz}$, thus falling beyond the maximum frequency of interest for this study.

This basic unit cell design functions as a building block for the annular compliant mechanism structure proposed in Figure 6.8. A three-dimensional finite element model of this structure was constructed using a two-dimensional continuum shell structure with two unit cells in parallel. This continuum model was then revolved 90 degrees about a predetermined axis to create a three-dimensional model. One quarter of the annular structure was modeled in order to make use of double-symmetry. The finite element model was built using approximately 80,000 solid elements and is shown in Figure 6.10. The purple elements in this figure represent the vulcanized rubber material; the green elements represent the spring steel material.

The computational analysis of the resultant 3-D annular structure was performed 


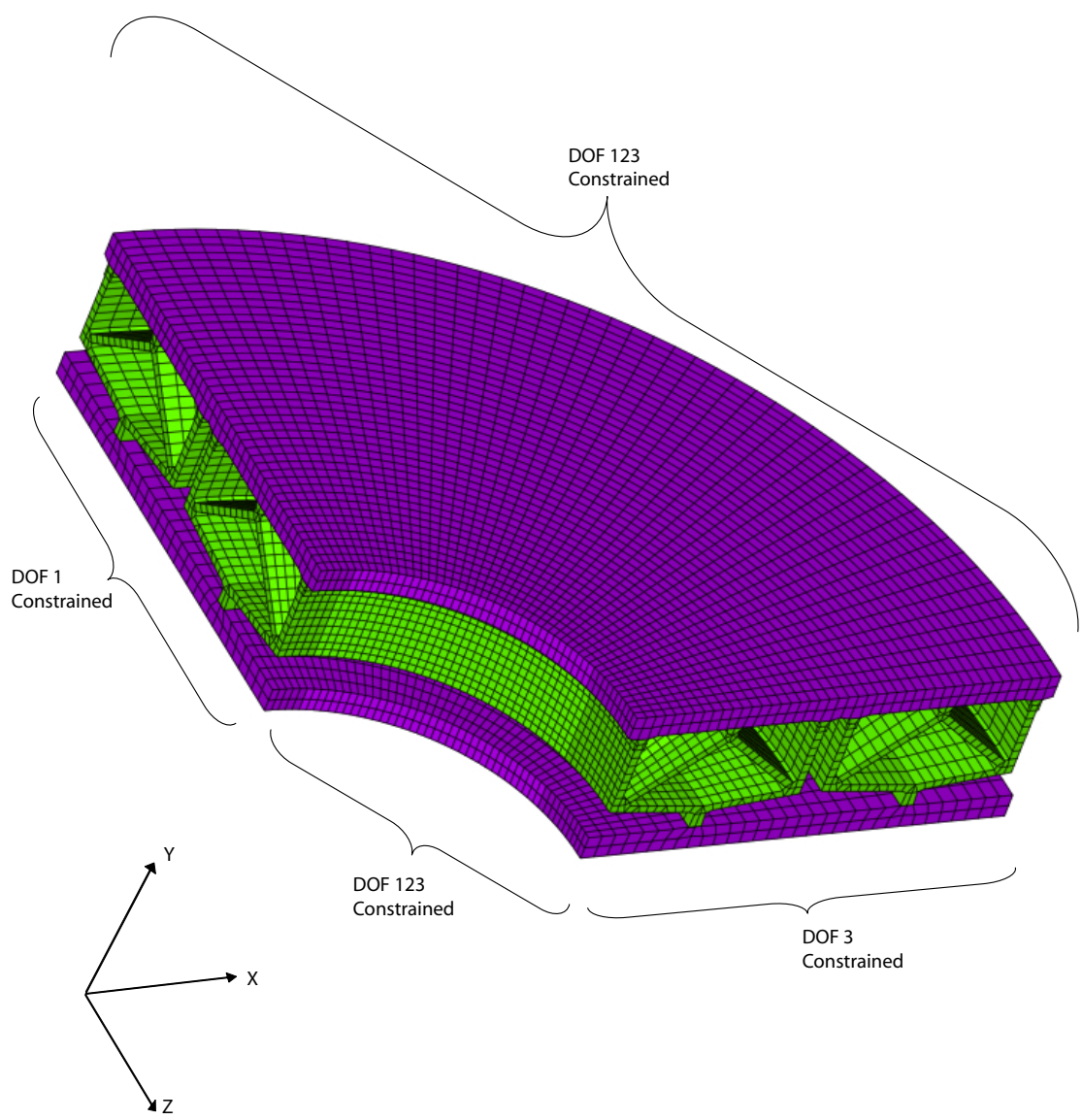

Figure 6.11: Boundary conditions for the finite element solid model of one quarter of the annular compliant mechanism structure

in Nastran. Specifically, two linear analyses were executed including a Direct Frequency Response analysis (SOL 108) and a Normal Modes analysis (SOL 103). Symmetric boundary conditions were applied to nodes laying along the radially extending surfaces of the quarter-model as shown in Figure 6.11. Observe that fixed-fixed boundary conditions were enforced by constraining the appropriate degrees of freedom of the nodes laying along the inner and outer circumferential boundaries of the model.

As depicted in Figure 6.12 (a), a unit magnitude distributed line load was applied 


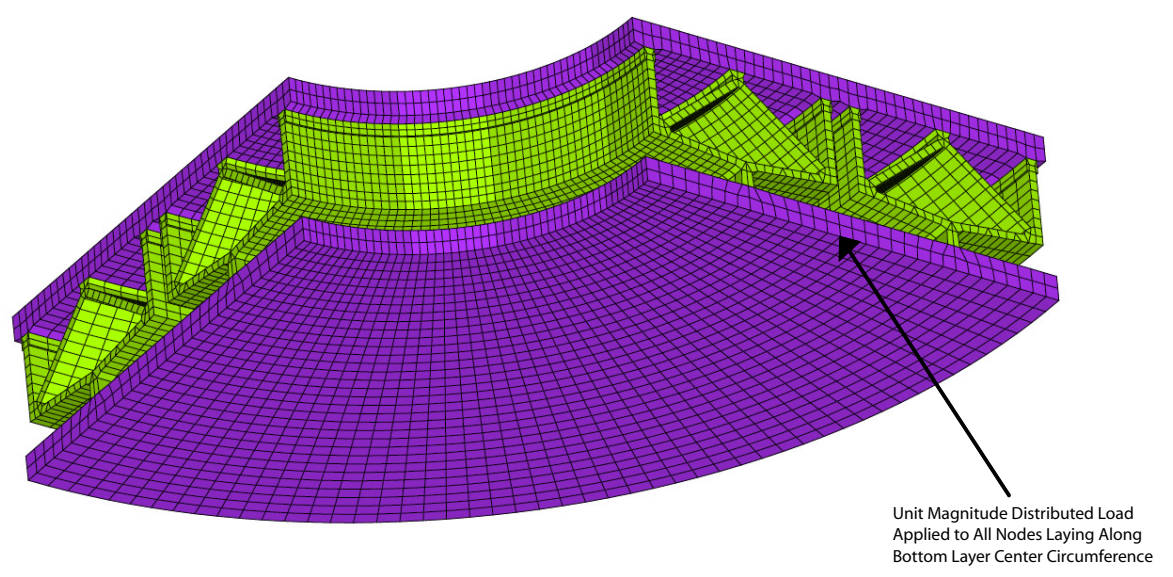

(a) Bottom layer loading

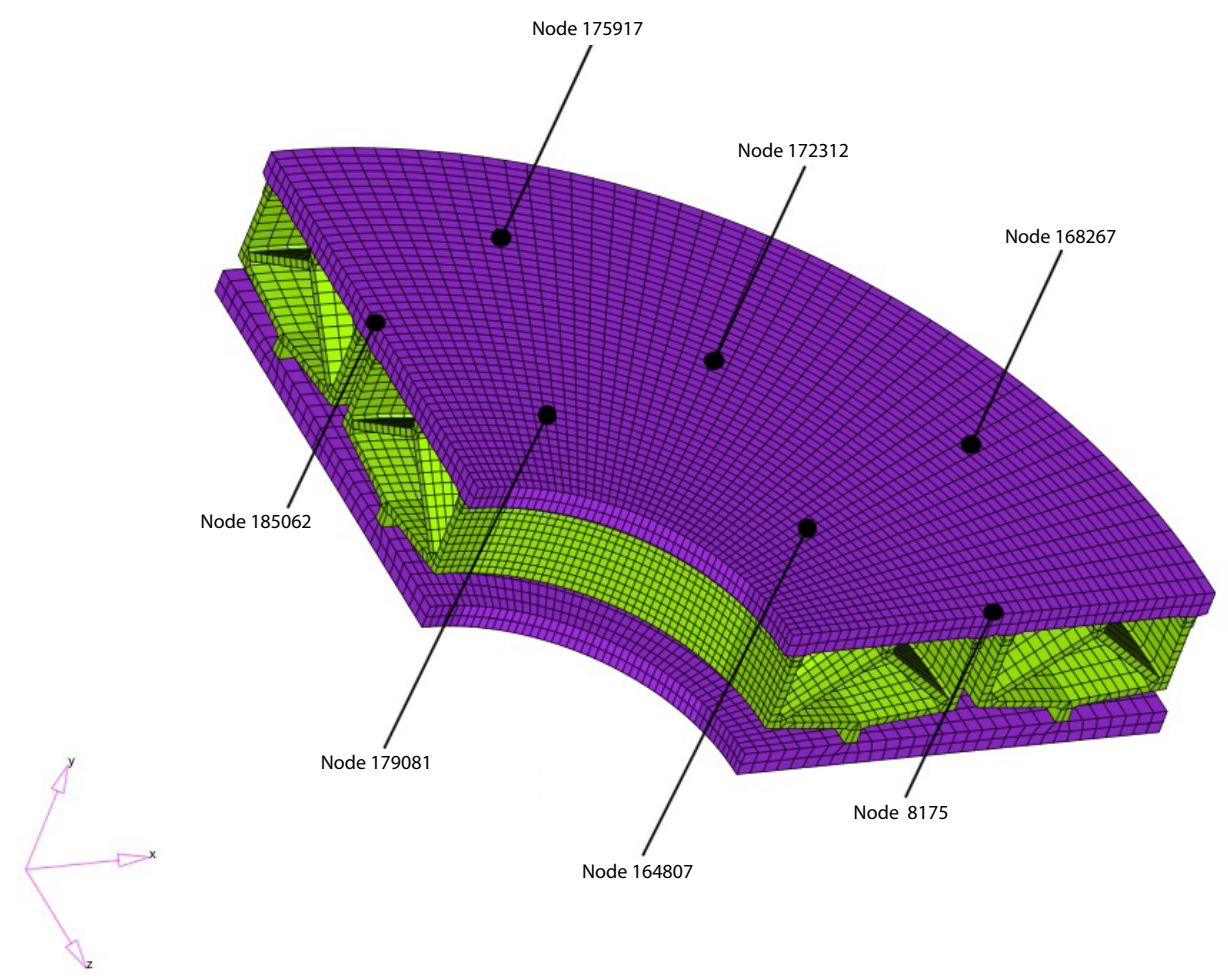

(b) Nodal locations for top layer response

Figure 6.12: Finite element solid model of one quarter of the annular compliant mechanism structure: (a) bottom layer loading and (b) nodal locations for top layer response 
to the bottom layer nodes laying along the center circumferential arc of the annular structure. The top layer velocity of the annular structure was monitored at the designated nodes illustrated through the top view perspective of the structure in Figure 6.12 (b). Observe that the locations of the monitored nodes correspond to the output of the inner compliant mechanism ring (nodes 164807 and 179081), the middle of the structure top layer (nodes 8175,172312 , and 185062), and the output of the outer compliant mechanism ring (nodes 168267 and 175917).

The top layer $y$-direction (i.e. normal) velocity of each of these seven nodes was computed using the Direct Frequency Response solution sequence over the frequency range of $5 \mathrm{~Hz}$ to $1 \mathrm{kHz}$, evaluated at $5 \mathrm{~Hz}$ intervals. The normal velocity at each node is shown in units of mm/s in Figure 6.13. Three resonant peaks occur at $200 \mathrm{~Hz}, 245$ $\mathrm{Hz}$, and $295 \mathrm{~Hz}$. At $300 \mathrm{~Hz}$ a sharp drop in response occurs at nodes that lay over the output of the inner compliant mechanism ring (i.e. nodes 164807 and 179081 in Figure 6.12 (b)). This drop signifies the start of the attenuation zone. Since the normal velocity at these two locations is smaller than the normal velocity at the other top layer nodal locations (at $300 \mathrm{~Hz}$ ), the connection scheme in Figure 6.8 is implemented where the pinion shaft is attached to the annular structure at the output location for the inner compliant mechanism ring.

This is further illustrated by comparing the computed RMS transverse velocity of the two output nodes for the inner compliant mechanism ring and the RMS transverse velocity of the remaining five nodes. While the RMS transverse velocity of the two output nodes for the inner compliant mechanism ring is slightly larger across the attenuation zone, the beginning of the attenuation zone at $300 \mathrm{~Hz}$ is distinct, as shown in Figure 6.14. Furthermore, response continues to drop off by approximately $20 \mathrm{~dB}$ as the attenuation zone is traversed. The RMS normal velocity response at 


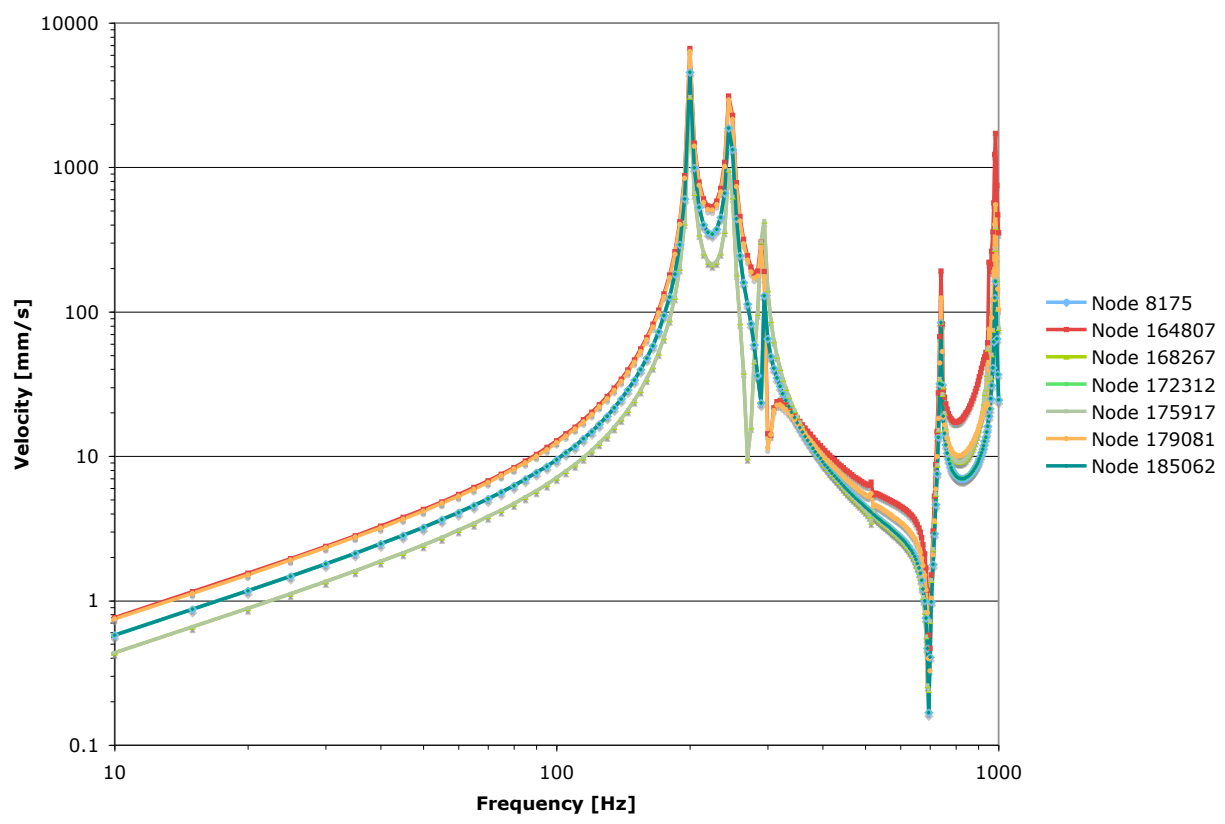

Figure 6.13: Top layer normal velocity of the annular compliant mechanism structure quarter model at the monitored nodal locations

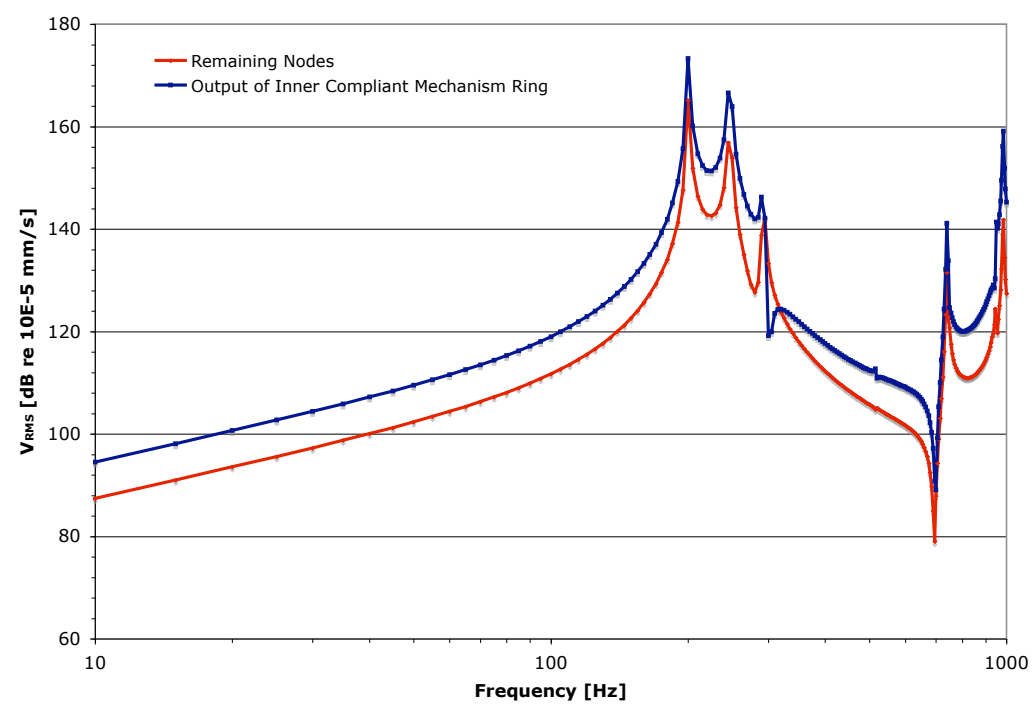

Figure 6.14: Top layer RMS velocity of the annular compliant mechanism structure quarter model 
the remaining five nodal locations follows the same trend. At approximately $740 \mathrm{~Hz}$ structural resonance occurs sooner than expected when compared with the unit cell analysis. This resonance represents the cutoff of the attenuation zone. Beyond this frequency, RMS normal velocity response increases with a maximum peak at $995 \mathrm{~Hz}$ just prior to the end of the frequency range of interest.

Examination of the mode shapes of the annular structure, shown in Figure 6.15, obtained from the Normal Modes analysis, reveals both the local and global behavior of the structure. In this figure, displacement contours are superimposed on the deformed shape of the structure. At $201 \mathrm{~Hz}$, Figure 6.15 (a), the first resonant mode of the structure is a global mode where the bottom layer moves circumferentially in unison with a half-wavelength deformation that causes motion of the structure top layer. The second global mode at $293 \mathrm{~Hz}$ occurs immediately before the attenuation zone. Large motion of the outer compliant mechanism ring is visible relative to the motion of the inner compliant mechanism ring, Figure 6.15 (b). At frequencies within the attenuation zone, Figure 6.15 (c) through (e), deformation and energy is confined to the rubber bottom layer. This bottom layer motion provides passive excitation to the compliant mechanism core leading to a reduction in top layer response via the principle of mechanical advantage. This phenomenon is clearly seen in Figures 6.16 (a) and (b) where the top and bottom rubber layers are hidden for clarity, revealing the fact that deformation is confined to the lower half of the steel core structure as a direct result of the chosen topology. Finally, moving to a frequency above of the attenuation zone (i.e. $990 \mathrm{~Hz}$ ), interaction between unit cells leads to the motion and greater energy content of the top layer causing significant increases in the normal velocity response of the annular structure, Figure 6.15 (f).

In summary, a comparison is made between the 2-D unit cell spectral finite element 


$$
\therefore 8
$$




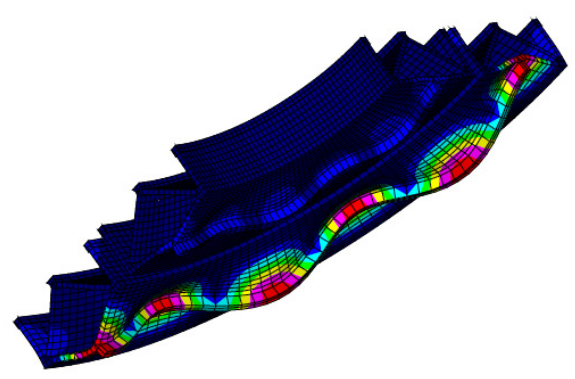

(a) $396 \mathrm{~Hz}$

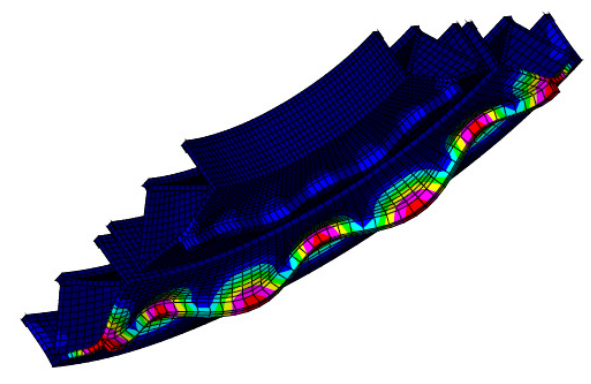

(b) $482 \mathrm{~Hz}$

Figure 6.16: Displacement contours superimposed on the normal mode shapes of the compliant mechanism core of one quarter of the annular compliant mechanism structure: (a) $396 \mathrm{~Hz}$ and (d) $482 \mathrm{~Hz}$

analysis and the 3-D conventional finite element continuum analysis of the quarter model annular structure. The local 2-D unit cell analysis adequately predicts the general structural dynamic response of the global structure. However, the unit cell analysis has limitations associated with predicting interactions between unit cells that result in resonances and a lower cutoff frequency at the global level.

From a broader perspective, all analyses presented in this section assume linear response. Since a rubber material is used, a more complete analysis should be considered to address the non-linear behavior of the annular compliant mechanism structure. Additionally, the details of the coupler design pertaining to the interface between the rubber top and bottom layers and the steel compliant mechanism core require attention in terms of physical implementation.

Despite these limitations and assumptions, the methodology from Section 6.1 is successfully applied to synthesize a preliminary design of a multi-material structure with integral compliant mechanisms for use within a vibration isolation coupler. This design is intended to isolate axial vibrations between two rotating shafts, however, 
a myriad of alternative design configurations exist for bushings (e.g. to reduce the transmission of the transverse vibrations of a shaft through a bushing to a housing) and couplers (e.g. to reduce torsional vibrations). These concepts represent other possible applications of the methodology summarized in this chapter. 


\section{CHAPTER 7}

\section{Conclusions}

In this final chapter areas of future research are set forth along with a summary of research achievements. Two new directions of investigation are proposed: 1) multimaterial structures for increased functionality and 2) adaptive-passive and active structures for variable gain response. In Section 7.1 each branch of study is briefly

outlined. Following this, initial concepts for structures having variable gain response are introduced in Section 7.2. In conclusion of this work a summary of research accomplishments is provided in Section 7.3.

\section{$7.1 \quad$ Future Work}

The use of multiple materials in the design of cellular or truss-like structures offers the combined advantages of static, dynamic, and thermal functionality at a reduced weight, as discussed by Wadley [101]. By coupling the analysis and optimization methodology (Chapter 6) with multi-material and/or fluid-filled compliant mechanism unit cell designs, applications in both traditional and emerging engineering disciplines can be explored. Specific areas of interest entail biomedical engineering applications including biological sensors and structures for environmental characterization, health monitoring, and biomaterial design. New approaches to the analysis of these structures are required in order to accurately describe the fluid-structure physics of the problem. The particle finite element computational method is a pos- 
sible approach to this branch of research, per Idelsohn et al. [44]. Furthermore, experimental validation of these computational models through the manufacturing and testing of such novel structures is another area of interest, see Gouker et al. [31].

Representing the second possible area of future work is the design of adaptivepassive and active structures with integral compliant mechanisms for variable gain response. Adaptive-passive and active structures adapt to their loading environment by automatically changing shape, and hence response, depending on the frequency of excitation. Several potential methods of modifying response exist including bi-stable compliant mechanism structures having multiple operating configurations, see for example Jensen \& Howell [46]. This approach will likely require a non-linear computational method coupled with an efficient tool for synthesizing appropriate compliant mechanism topologies. Additionally, the use of actuators and active control, or the use of adaptable materials (e.g. magnetorheological (MR), electrostrictive (ER), or shape memory alloy (SMA) materials) have potential for use in realizing variable gain response. The next section focuses on this research area and gives an overview of some of the essential concepts that are fundamental to the development of structures having variable gain response.

\subsection{Structures having Variable Gain Response}

Building on the research completed for the attenuation of structural vibration within fixed frequency ranges, the goal of this section is to examine how the variation of three different unit cell parameters (i.e. bottom layer stiffness, bottom layer mass, and periodicity) modify the wavenumber-frequency relationship and subsequent unit cell structural dynamic response. Additionally, the use of internal actuators for the variation of the amplification properties of the compliant mechanism unit cell is 
addressed. The manifestation of such variations through actual physical changes to the unit cell is discussed. Thus, this section covers the fundamental methods by which structures having variable gain response can be realized. The limitations of these methods are also discussed in relation to what is feasible in the construction of physical prototypes.

The effect of unit cell bottom layer stiffness, bottom layer mass, and periodicity on dynamic response is clarified by re-examining the unit cell wavenumber-frequency relationship, Eqn (3.16), from Chapter 3.

$$
\omega=\left(\frac{2 \pi}{\lambda}\right)^{2} \sqrt{\frac{E I}{\rho A}}
$$

Recall that $\rho, E, A$, and $I$ are the physical parameters of the unit cell bottom layer beam. Additionally, $\omega$ is the frequency of vibration, and $\lambda$ is the wavelength of transverse waves propagating through the bottom layer beam.

Inspection of Eqn (3.16) makes clear three key relationships by examining in turn the variation in $\omega$ due to a variation in the unit cell material Young's modulus (i.e. bottom layer stiffness), material density (i.e. bottom layer mass), or wavelength (i.e. unit cell periodicity). Observe that the term "unit cell periodicity" refers to the wavelength, length scale, or width of the unit cell and that, at the frequency at which attenuation begins, all of these are equal.

By holding $\rho, A, I$, and $\lambda$ constant in Eqn (3.16) and introducing a normalized Young's modulus, $E_{\text {norm }}$, the effect of changing the actual Young's modulus, E, of the unit cell bottom layer is examined

$$
\omega=C_{1} \sqrt{\frac{E}{E_{\text {norm }}}}
$$

where the constant, $C_{1}$, is defined as

$$
C_{1}=\left(\frac{2 \pi}{\lambda}\right)^{2} \sqrt{\frac{E_{\text {norm }} I}{\rho A}}
$$


Rewriting Eqn 7.1 allows for the definition of a stiffness normalized frequency, $\omega_{E \_n o r m}$, to be written in terms of the normalized Young's modulus and actual Young's modulus of the unit cell bottom layer.

$$
\omega_{E \_n o r m}=\frac{\omega}{C_{1}}=\sqrt{\frac{E}{E_{\text {norm }}}}
$$

A similar relationship is derived for a mass normalized frequency, $\omega_{\rho_{-} n o r m}$, in terms of a normalized density, $\rho_{\text {norm }}$, and the actual density of the unit cell bottom layer.

$$
\omega=C_{2} \sqrt{\frac{\rho_{\text {norm }}}{\rho}}
$$

where the constant, $C_{2}$, is defined as

$$
C_{2}=\left(\frac{2 \pi}{\lambda}\right)^{2} \sqrt{\frac{E I}{\rho_{\text {norm }} A}}
$$

giving

$$
\omega_{\rho_{-n o r m}}=\frac{\omega}{C_{2}}=\sqrt{\frac{\rho_{\text {norm }}}{\rho}}
$$

A relationship is also derived for a periodicity normalized frequency, $\omega_{\lambda_{-} \text {norm }}$, in terms of a normalized wavelength, $\lambda_{\text {norm }}$, and the actual wavelength of vibration for the unit cell bottom layer.

$$
\omega=C_{3}\left(\frac{\lambda_{\text {norm }}}{\lambda}\right)^{2}
$$

where the constant, $C_{3}$, is defined as

$$
C_{3}=\left(\frac{2 \pi}{\lambda_{\text {norm }}}\right)^{2} \sqrt{\frac{E I}{\rho A}}
$$

giving the final relationship of interest

$$
\omega_{\lambda_{-} \text {norm }}=\frac{\omega}{C_{3}}=\left(\frac{\lambda_{\text {norm }}}{\lambda}\right)^{2}
$$




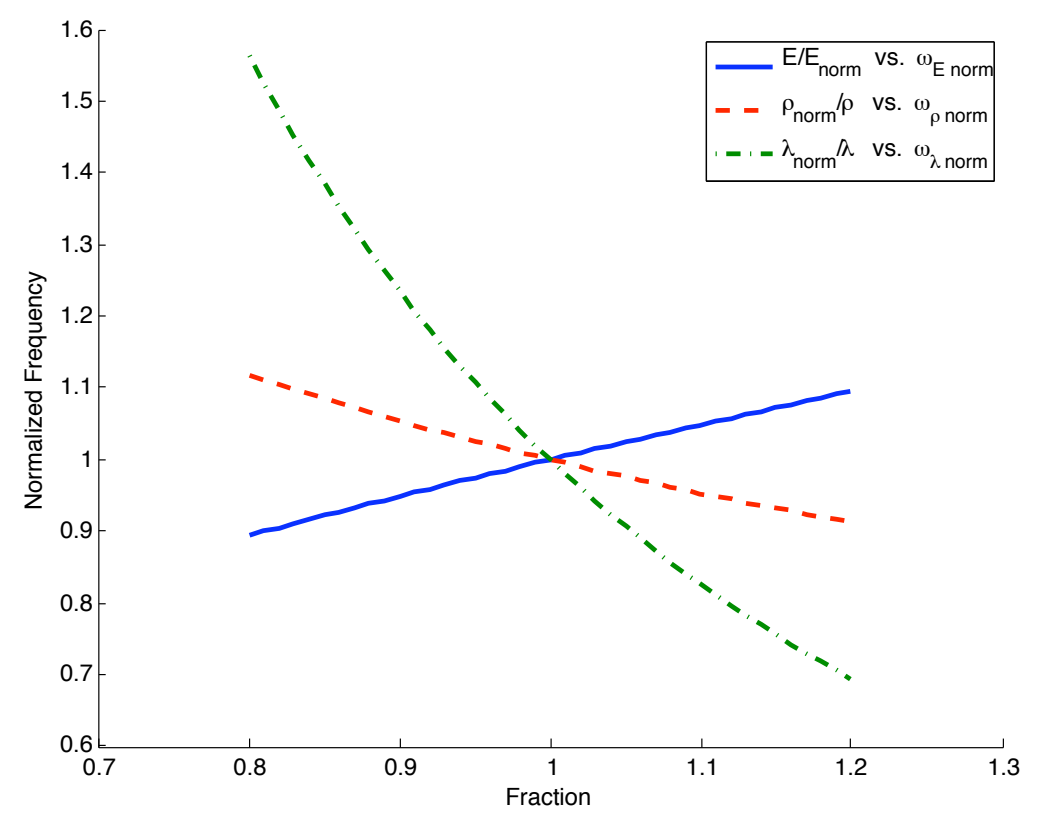

Figure 7.1: Normalized frequency as a function of a fraction of the normalized unit cell bottom layer stiffness, bottom layer mass, and periodicity

The normalized frequency relationships in Eqns (7.3)-(7.9) are now computed by setting the variables $E, \rho$, and $\lambda$ to some fraction of $E_{n o r m}, \rho_{n o r m}$, and $\lambda_{n o r m}$, respectively. Figure 7.1 illustrates each relationship using fractions ranging between 0.8 and 1.2. This figure makes clear the required modification in either bottom layer stiffness, bottom layer mass, or unit cell periodicity required to achieve either an increase or decrease in the attenuation zone starting frequency. Assuming all other variables are held constant, increasing the attenuation zone starting frequency equates to an increase in the unit cell bottom layer stiffness, a decrease in the unit cell bottom layer mass, or a reduction in the length scale of the unit cell (i.e. an increase in periodicity). Alternatively, a decrease in the attenuation zone starting frequency equates to a decrease in the unit cell bottom layer stiffness, an increase in the unit cell bottom layer mass, or an increase in the length scale of the unit cell (i.e. 
a reduction in periodicity). Moreover, Figure 7.1 illustrates that the most effective means of varying the start of the attenuation zone is to adjust the length scale (i.e. periodicity) of the unit cell since $\omega_{\lambda_{-} n o r m}$ varies with a $1 / \lambda^{2}$ relationship.

In contrast to varying the beginning of the attenuation zone, recall that the end of the attenuation zone depends on the selection of a compliant mechanism core topology and size as discussed in Chapters 3 and 4 . Thus, by understanding the underlying methods through which an attenuation zone is controlled, effective ways of adapting or varying the vibration characteristics of the unit cell can be formulated. In the subsequent three sections, the response of the rhombus compliant mechanism unit cell from Section 3.2.4 is examined by varying the unit cell bottom layer stiffness, bottom layer mass, or periodicity.

\subsubsection{Unit Cell Bottom Layer Stiffness}

The top layer RMS transverse velocity of the rhombus compliant mechanism unit cell was computed using three different values for the unit cell bottom layer material Young's modulus, E, while keeping all other analysis parameters constant. While directly varying the material Young's modulus in this manner is not physically possible, this study serves a purpose in showing the effect of increasing bottom layer stiffness on wave propagation and the subsequent unit cell structural dynamic response. Young's modulus (i.e. $E$ ) values of $0.9 \times E_{\text {norm }}, 1.0 \times E_{\text {norm }}$, and $1.1 \times E_{\text {norm }}$ were respectively used for the unit cell bottom layer beam in Eqn (7.3). The unit cell top layer RMS normal velocity response shown in Figure 7.2 was computed from $0 \mathrm{~Hz}$ to $15 \mathrm{kHz}$ at $10 \mathrm{~Hz}$ frequency intervals. This figure demonstrates a clear relationship between bottom layer stiffness and the beginning of both attenuation zones. As stiffness increases the attenuation starting frequency shifts towards higher frequencies and shorter wavelengths. 


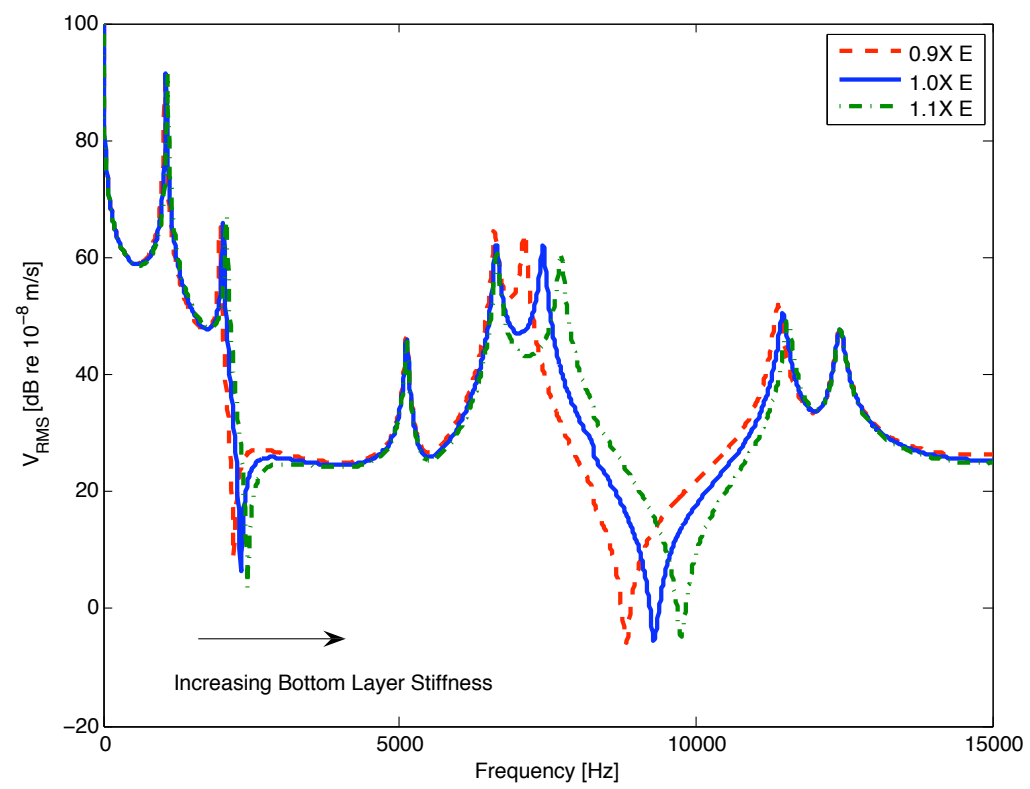

Figure 7.2: Relationship between unit cell response and increased bottom layer stiffness

Modifying the Young's modulus of the unit cell bottom layer, as done in this conceptual study, is not physically feasible. Nonetheless, other methods for realizing such an effect are possible. Specifically, the use of a composite beam for the bottom layer of the unit cell is worth further investigation. This bottom layer would consist of a MR material layer sandwiched between, and contained by, two elastic material layers as suggested by several researchers including Harland et al. [34, 35] and Yalcintas \& Dai [105]. This concept is shown in Figure 7.3.

The primary assumptions pertaining to the bottom layer of this model are: 1) there is no slip between the elastic layers and the MR layer; 2) all three layers experience the same transverse deflection; 3) no normal stresses exist in the MR layer; and 4) no shear stresses exist in the elastic layers, per Yalcintas \& Dai [105]. As a consequence of these assumptions, an increase in the shear modulus of the MR layer will lead to an increase in the bending stiffness of the composite beam. 


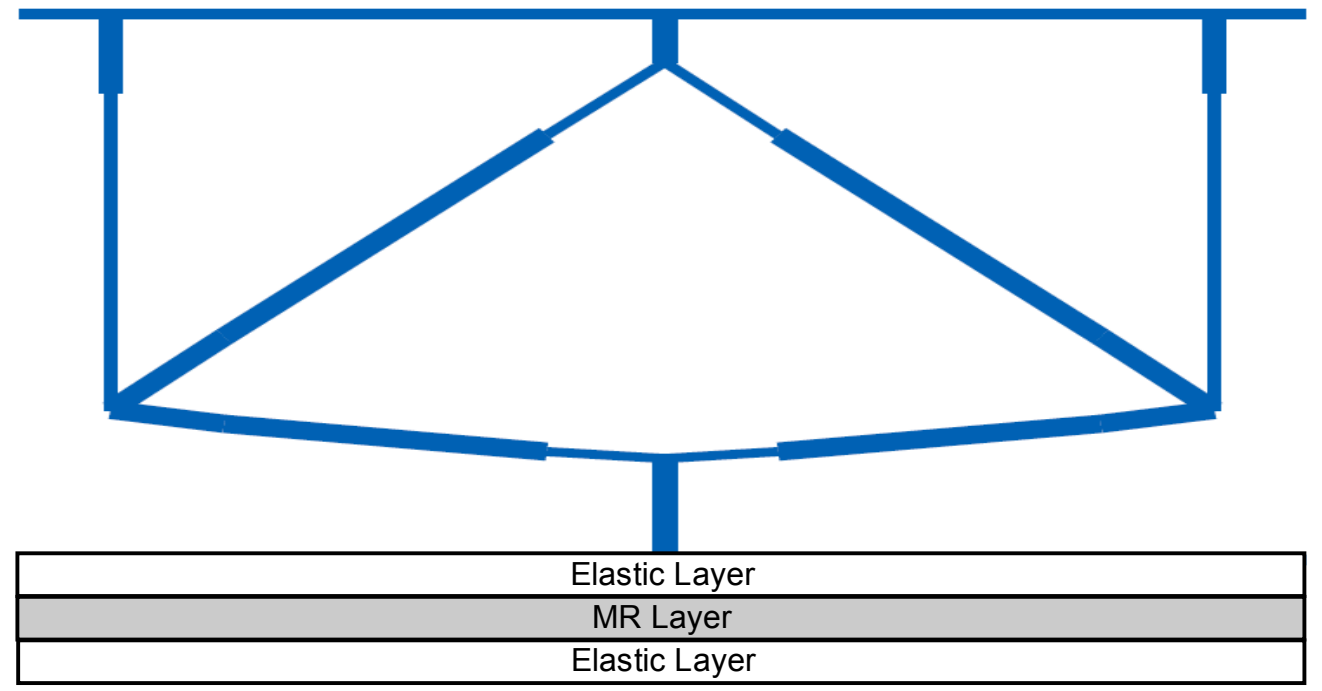

Figure 7.3: Conceptual model of a compliant mechanism unit cell having a composite bottom layer designed for variable gain response

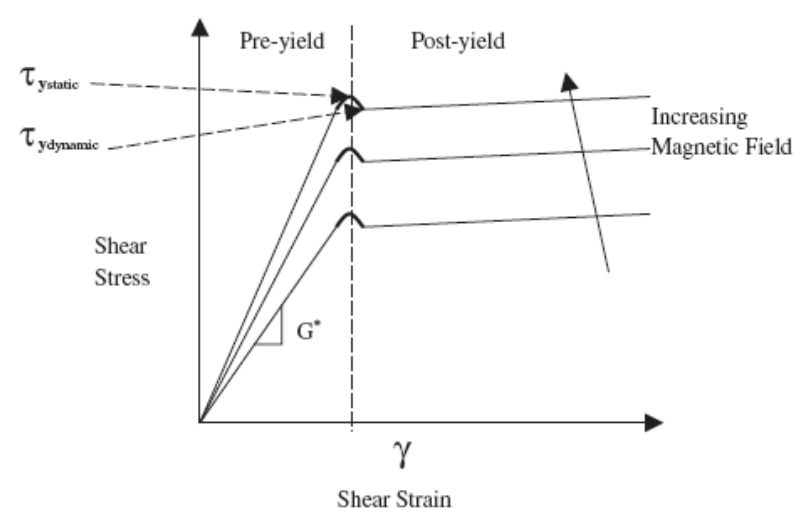

(a) Shear stress-shear strain relationship

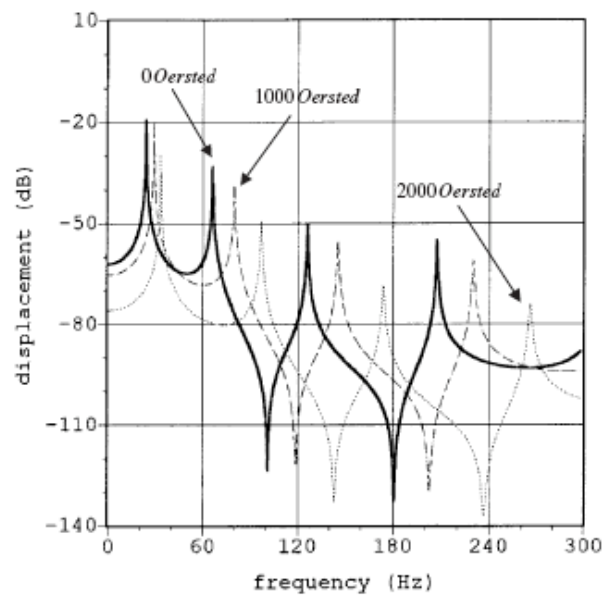

(b) Transverse vibration response

Figure 7.4: MR material behavior and composite beam structural dynamic response: (a) shear stress-shear strain relationship for MR materials, and (b) transverse vibration response for MR composite beam, from Yalcintas \& Dai [105] 
The physical properties (i.e. viscosity and shear modulus) of MR materials vary when the material is subjected to different magnetic field levels. For example, the shear stress-shear strain relationship for MR materials is shown qualitatively in Figure 7.4 (a). Assuming behavior within the linear pre-yield region of this figure and modifying the externally applied magnetic field level, variations in the stiffness and damping properties of the unit cell composite bottom layer are realized. In theory, these variations will allow control over the structural dynamic response of the unit cell. Specifically, the beginning of the attenuation zone is modified by the application of a specific magnetic field level similar to the variations in dynamic response predicted by Yalcintas \& Dai [105] for a simple composite MR beam shown in Figure 7.4 (b).

\subsubsection{Unit Cell Bottom Layer Mass}

The effect of varying the unit cell bottom layer material density, $\rho$, is examined in this section using three different values for the density while holding all other analysis parameters constant. Density (i.e. $\rho$ ) values of $0.9 \times \rho_{\text {norm }}, 1.0 \times \rho_{\text {norm }}$, and $1.1 \times \rho_{\text {norm }}$ were respectively used for the unit cell bottom layer beam in Eqn (7.6). The unit cell top layer RMS normal velocity response shown in Figure 7.5 was computed from $0 \mathrm{~Hz}$ to $15 \mathrm{kHz}$ at $10 \mathrm{~Hz}$ frequency intervals. This figure illustrates that a decrease in the mass of the bottom layer of the unit cell, exclusive of a change in any other analysis parameter, has the equivalent effect as an increase in the stiffness of the unit cell bottom layer.

From an implementation perspective, the approach of adding point masses to the unit cell bottom layer has potential for reducing structural vibration as established by the work of Martinsson \& Movchan [65]. However, physical means of easily adding or removing point masses to the unit cell bottom layer generally do not exist. 


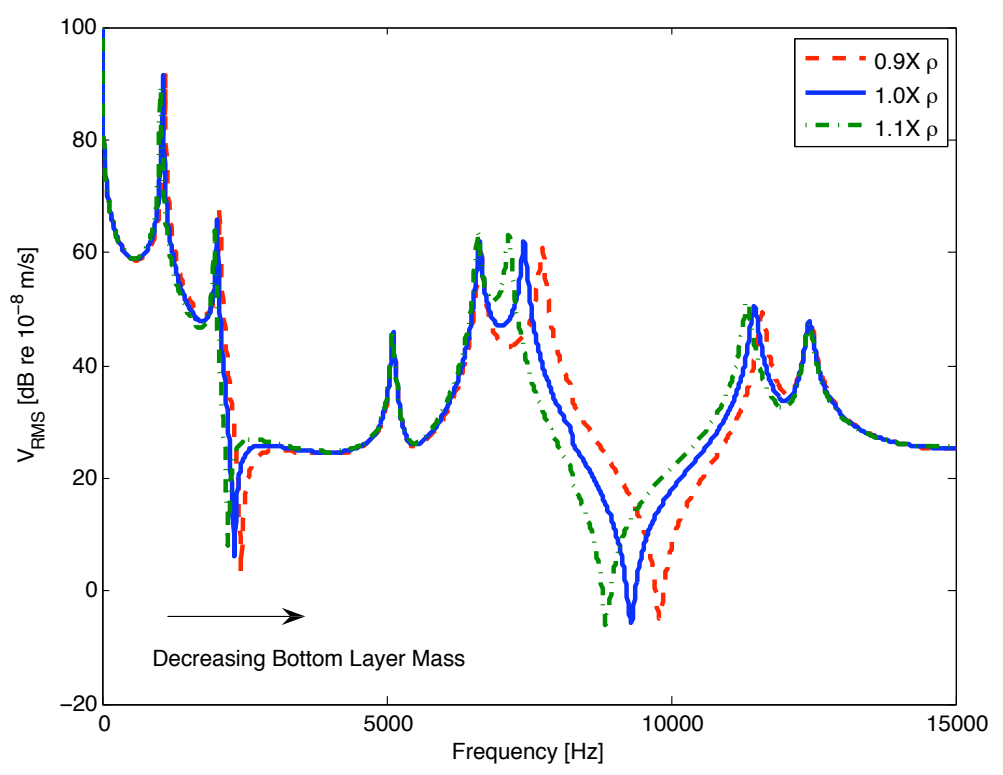

Figure 7.5: Relationship between unit cell response and decreased bottom layer mass

Alternatively, using a thicker (i.e. more massive) bottom layer beam would also increase the cross-section area moment of inertia, I, in Eqn (3.16), which has the opposite result of the added mass. Therefore, while the results of Martinsson \& Movchan and the results of this study are conceptually interesting, their practical utility in relation to designing structures that have variable gain response is not very high.

\subsubsection{Unit Cell Periodicity}

The effect of varying the periodicity of the unit cell within a global structure is studied through the modification of the length of the unit cell. The concept for a unit cell having different lengths is illustrated in Figure 7.6, where length, or wavelength (i.e. $\lambda$ ), values of $0.9 \times \lambda_{\text {norm }}, 1.0 \times \lambda_{\text {norm }}$, and $1.1 \times \lambda_{\text {norm }}$ are shown. Observe that $\lambda$ is respectively set to each of these values in Eqn (7.9) to determine the starting frequency of the attenuation zone. The top layer RMS normal velocity response of 


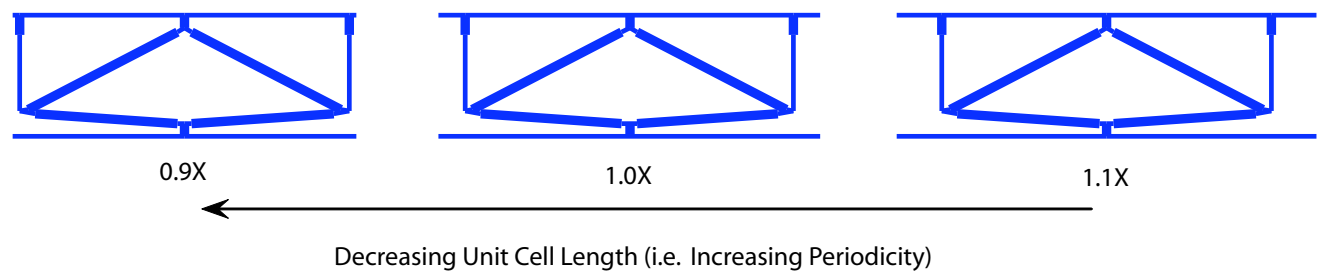

Figure 7.6: Graphical representation of the modification of unit cell periodicity

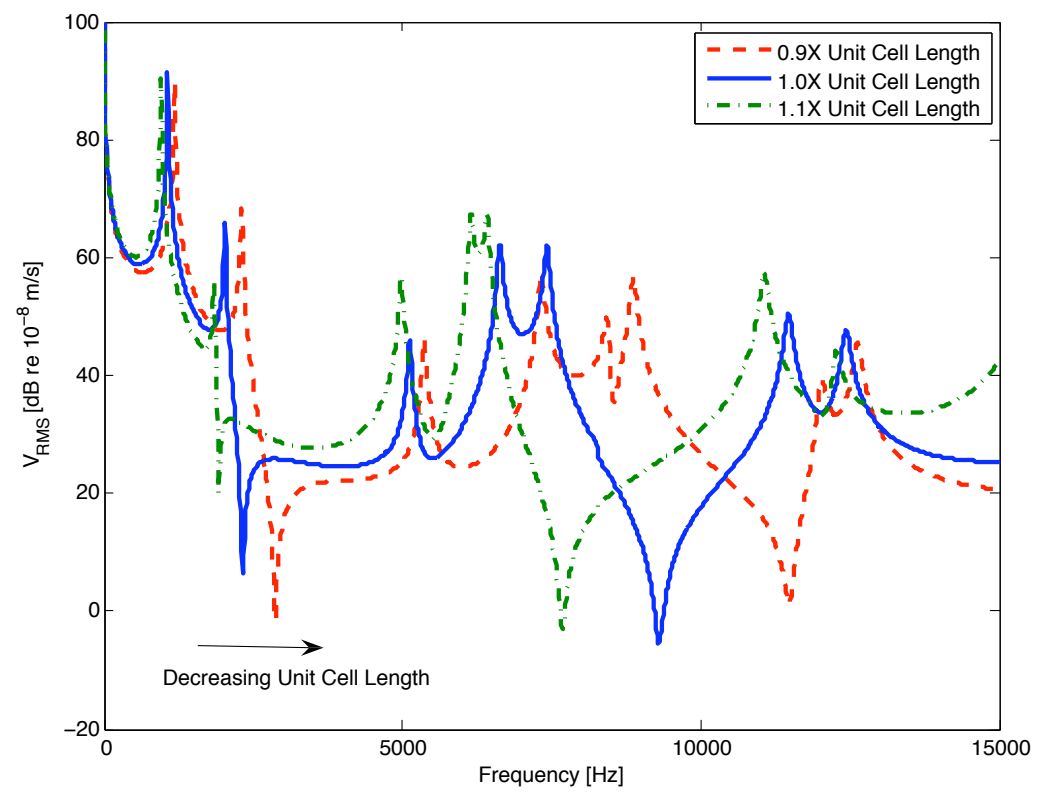

Figure 7.7: Relationship between unit cell response and decreased unit cell length (i.e. periodicity)

each different length unit cell is shown in Figure 7.7. Vibratory response was once more computed from $0 \mathrm{~Hz}$ to $15 \mathrm{kHz}$ at $10 \mathrm{~Hz}$ frequency intervals. Significant shifting of the attenuation zone starting frequency towards higher frequencies is evident as the length of the unit cell is decreased. Thus, as the spacing between compliant mechanism unit cells within a global structure is reduced, the vibration reduction 
characteristics will accordingly shift towards increasingly higher frequencies.

In terms of the physical implementation of the shape changes shown in Figure 7.6, the latest research being performed in the field of SMA materials has significant potential. Specifically, materials such as NiTi alloy metals (e.g. "Nitinol") have unique thermomechanical properties that can potentially be exploited in realizing global shape changes, plus changes to the material stiffness and damping properties. Shaw \& Kyriakides [91] have thoroughly investigated the relationships between temperature, material phase, and mechanical properties. They have shown that the high temperature Austenite phase of NiTi has a much larger storage modulus and lower loss modulus than the room temperature twinned Martensite phase. Thus, considering a constant shape SMA compliant mechanism structure, a higher operating temperature (i.e. a temperature above the Martensite to Austenite phase transition temperature) will result in stiffer dynamic response that exhibits less internal damping. Consequently, controlling the external thermal environment of a NiTi metal compliant mechanism structure, or passing temperature controlled fluid through the internal compliant mechanism topology, could provide substantial dynamic performance control capability.

Taking this SMA material research a step further, Grummon, Shaw, \& Foltz [32] have recently developed a new method for brazing NiTi materials using niobium. They have demonstrated that complex cellular topologies can be formed using NiTi material as shown in Figure 7.8. Additionally, this technology allows the application of large recoverable strain to these structures, resulting in substantial modification of the structural geometry as shown in Figure 7.9. Thus, the ability to reliably manufacture such structures, coupled with their outstanding mechanical performance and unique thermomechanical material behavior, points to several new directions for 


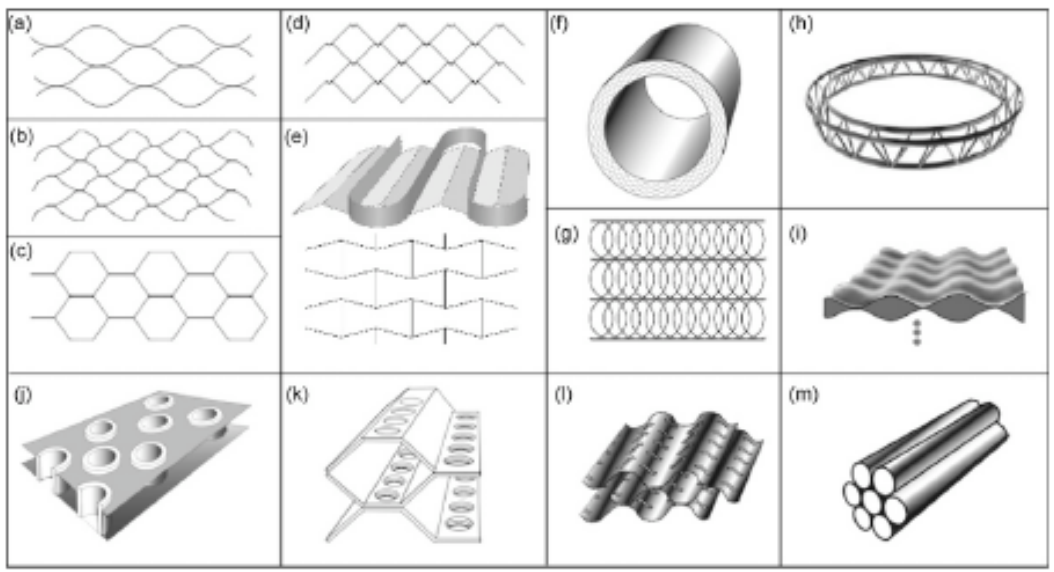

Figure 7.8: Cellular topologies enabled using new NiTi brazing method, from Grummon, Shaw, \& Foltz [32]

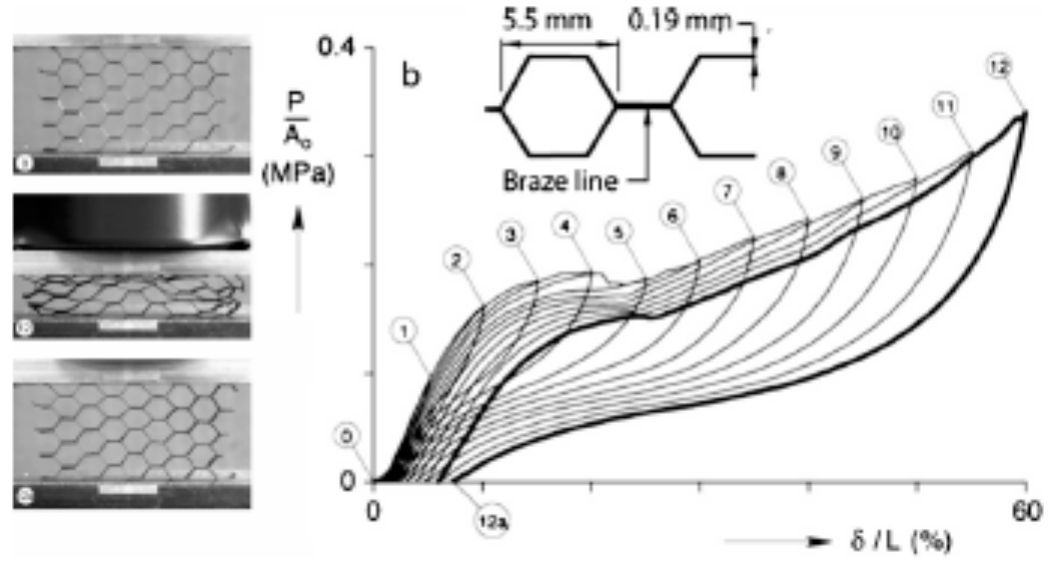

Figure 7.9: Mechanical response of a niobium-brazed open cell honeycomb structure built from NiTi strip material, from Grummon, Shaw, \& Foltz [32] 


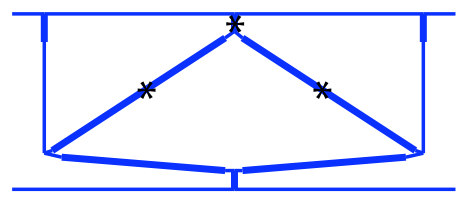

(a) $\mathrm{MA}=7$

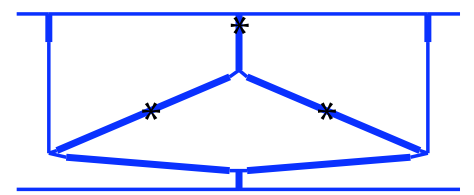

(b) $\mathrm{MA}=5$

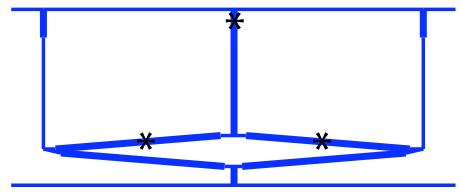

(c) $\mathrm{MA}=1$

Figure 7.10: Rhombus compliant mechanism unit cells with varying mechanical advantage: (a) $\mathrm{MA}=7$, (b) $\mathrm{MA}=5$, and (c) $\mathrm{MA}=1$ (asterisks denote locations of internal actuators)

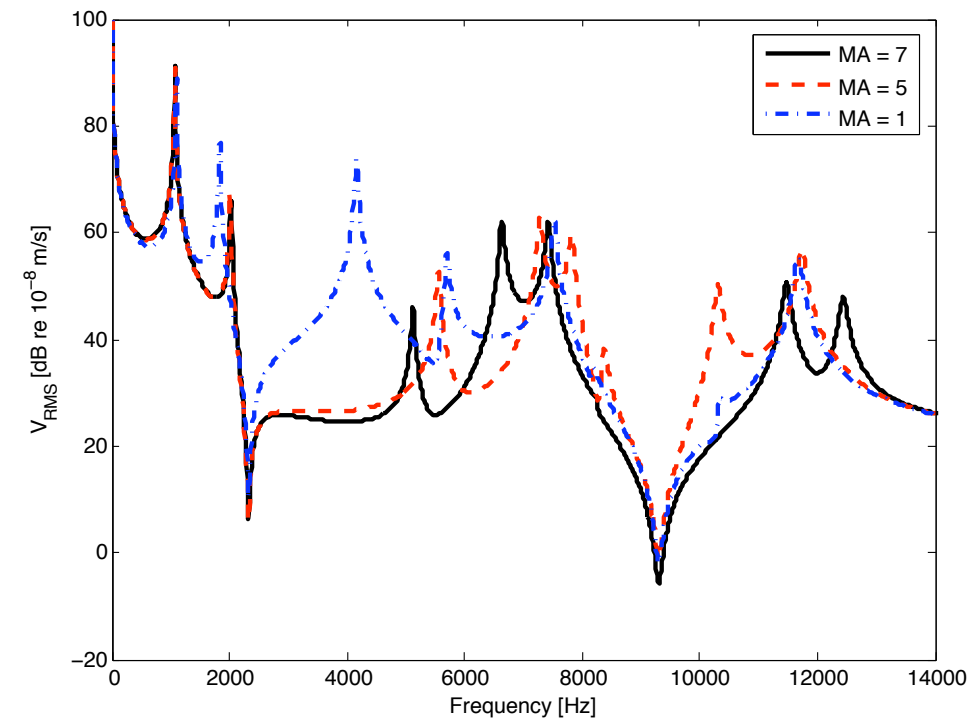

Figure 7.11: Top layer RMS velocity comparison for rhombus compliant mechanism unit cells with varying mechanical advantage due to internal shape change via actuators

future research.

\subsubsection{Internal Actuators}

In this final section concerned with variable gain response the addition of internal actuators to the rhombus compliant mechanism unit cell is briefly discussed. A portion of the results from Section 3.2.5 are revisited in Figures 7.10 (a) through (c) and Figure 7.11. In Figures 7.10 (a) through (c), three rhombus compliant mechanism unit cells are shown having mechanical advantage values of 7,5 , and 1 , respectively. 
The location of unit cell internal actuators are denoted by asterisks in these figures. These actuators are necessary to realize the illustrated shape changes.

By changing the internal shape of the compliant mechanism the breadth of the attenuation zone is modified as illustrated in Figure 7.11. If, for example, variable gain response is sought at a frequency of $4 \mathrm{kHz}$, the modification of the amplification properties of the compliant mechanism unit cell through an internal shape change results in the desired control. Such an approach coupled with any of the concepts presented in Sections 7.2.1 through 7.2.3 would provide enhanced variable gain response capabilities.

\subsection{Summary}

In this dissertation numerical methods were synthesized for the computational analysis of structures with integral compliant mechanisms. These methods were applied to the conceptualization, design, and optimization of novel two-dimensional and three-dimensional structures for reduced vibro-acoustic response. The central tools necessary for bringing together an overarching methodology were formulated in Chapter 3. These tools include: 1) a mechanical advantage framework for a priori mechanism topology and shape synthesis; 2) a wavenumber-frequency analysis for predicting response attenuation zones; 3) the definition of a compliant mechanism unit cell FRF; and 4) a deformed shape and energy distribution analysis to facilitate fundamental understanding of the physical behavior and energy flow through a structure.

Following the introduction of these initial concepts, a unit cell design using an alternative polymer material was examined to show the connection between material selection, structure size, and attenuation zone placement. The initial concepts were 
then applied to the design of parallel and serial connected structures in order to gain a thorough understanding of the additive composite effect of connecting either the same or different unit cells together in various configurations. Methods of increasing vibro-acoustic isolation were discussed along with the design of structures having multi-functional properties. Unit cell size and topology optimization using a genetic algorithm was then brought forth in Chapter 4 as a supplementary tool for structural dynamic response tuning and the realization of alternative topologies. Throughout all of these studies, the spectral finite element method proved to be a practical tool in the exploration of these ideas, and it noticeably reduced the complexity, while increasing the reliability, of such analyses.

Experimental investigations were performed to establish the validity of the underlying hypotheses behind this work. Limitations were discussed regarding the capability of a spectral finite element analysis in predicting the response of two prototype continuum structures. As a result, a conventional finite element analysis was required and performed using industry standard software to verify experimental results. Chapter 5 provided the results from this experimental work, which validated the hypotheses proposed at the conception of this research effort. Specifically, physically realizable structures having enhanced attenuation zones were designed, manufactured, tested, and shown to perform as expected.

An overview of the entire methodology was presented in Chapter 6. The utility of the methodology was illustrated through the development of two primary applications including a vibro-acoustic structural chassis/lining for an aerospace vehicle, and an axial vibration isolation coupler for an automotive vehicle drive shaft. The second application highlighted the techniques developed in Chapter 3 through the design of a 3-D annular (i.e. ring-like) structure. Furthermore, this application 
also emphasized the use of conventional solid finite elements in the computational modeling of a generally more complicated structure.

Finally, in this chapter potential extensions of this research towards the development of multi-material/functional, adaptive-passive, or active structures were described. Fundamental connections were drawn between various unit cell parameters and the vibratory behavior of the unit cell. These connections should serve as the starting point for future research and should be exploited in realizing global structures with variable gain response. Many promising future directions for this research exist based on the initial steps taken in this dissertation. Moreover, this effort serves as a basis for more sophisticated computational verification and experimental validation. Interesting applications and broader manufacturing approaches at smaller scales appear possible when the scope of this work is broadened to encompass analysis in the non-linear regime, along with the exclusive or combined use of multiple materials such as polymers, composites, biological tissues, and adaptable materials. 


\section{APPENDICES}




\section{APPENDIX A}

\section{Stiffness Matrix Components for Spectral Beam Element}

$$
\hat{K}_{\mathrm{b}_{11}}=\frac{(1+\mathrm{i})\left(-\mathrm{i}+\mathrm{e}^{2 \mathrm{i} k L}-\mathrm{e}^{2 k L}+\mathrm{ie}^{(2+2 \mathrm{i}) k L}\right) k^{3}}{1+\mathrm{e}^{2 \mathrm{i} k L}-4 \mathrm{e}^{(1+\mathrm{i}) k L}+\mathrm{e}^{2 k L}+\mathrm{e}^{(2+2 \mathrm{i}) k L}}
$$

$$
\hat{K}_{\mathrm{b}_{12}}=\frac{\mathrm{i}\left(-1+\mathrm{e}^{2 \mathrm{i} k L}\right)\left(-1+\mathrm{e}^{2 k L}\right) k^{2}}{1+\mathrm{e}^{2 \mathrm{i} k L}-4 \mathrm{e}^{(1+\mathrm{i}) k L}+\mathrm{e}^{2 k L}+\mathrm{e}^{(2+2 \mathrm{i}) k L}}
$$

$$
\hat{K}_{\mathrm{b}_{13}}=\frac{2\left(-\mathrm{e}^{\mathrm{i} k L}+\mathrm{ie}^{k L}-\mathrm{ie}^{(1+2 \mathrm{i}) k L}+\mathrm{e}^{(2+\mathrm{i}) k L}\right) k^{3}}{1+\mathrm{e}^{2 \mathrm{i} k L}-4 \mathrm{e}^{(1+\mathrm{i}) k L}+\mathrm{e}^{2 k L}+\mathrm{e}^{(2+2 \mathrm{i}) k L}}
$$

$$
\hat{K}_{\mathrm{b}_{14}}=\frac{2\left(-\mathrm{e}^{\mathrm{i} k L}+\mathrm{e}^{k L}+\mathrm{e}^{(1+2 \mathrm{i}) k L}-\mathrm{e}^{(2+\mathrm{i}) k L}\right) k^{2}}{1+\mathrm{e}^{2 \mathrm{i} k L}-4 \mathrm{e}^{(1+\mathrm{i}) k L}+\mathrm{e}^{2 k L}+\mathrm{e}^{(2+2 \mathrm{i}) k L}}
$$

$$
\hat{K}_{\mathrm{b}_{22}}=\frac{(1+\mathrm{i})\left(-1+\mathrm{ie}^{2 \mathrm{i} k L}-\mathrm{ie}^{2 k L}+\mathrm{e}^{(2+2 \mathrm{i}) k L}\right) k}{1+\mathrm{e}^{2 \mathrm{i} k L}-4 \mathrm{e}^{(1+\mathrm{i}) k L}+\mathrm{e}^{2 k L}+\mathrm{e}^{(2+2 \mathrm{i}) k L}}
$$

$$
\hat{K}_{\mathrm{b}_{23}}=-\frac{2\left(-\mathrm{e}^{\mathrm{i} k L}+\mathrm{e}^{k L}+\mathrm{e}^{(1+2 \mathrm{i}) k L}-\mathrm{e}^{(2+\mathrm{i}) k L}\right) k^{2}}{1+\mathrm{e}^{2 \mathrm{i} k L}-4 \mathrm{e}^{(1+\mathrm{i}) k L}+\mathrm{e}^{2 k L}+\mathrm{e}^{(2+2 \mathrm{i}) k L}}
$$

$$
\hat{K}_{\mathrm{b}_{24}}=\frac{2\left(\mathrm{e}^{\mathrm{i} k L}+\mathrm{ie}^{k L}-\mathrm{ie}^{(1+2 \mathrm{i}) k L}-\mathrm{e}^{(2+\mathrm{i}) k L}\right) k}{1+\mathrm{e}^{2 \mathrm{i} k L}-4 \mathrm{e}^{(1+\mathrm{i}) k L}+\mathrm{e}^{2 k L}+\mathrm{e}^{(2+2 \mathrm{i}) k L}}
$$

$$
\hat{K}_{\mathrm{b}_{33}}=\frac{(1+\mathrm{i})\left(-\mathrm{i}+\mathrm{e}^{2 \mathrm{i} k L}-\mathrm{e}^{2 k L}+\mathrm{ie}^{(2+2 \mathrm{i}) k L}\right) k^{3}}{1+\mathrm{e}^{2 \mathrm{i} k L}-4 \mathrm{e}^{(1+\mathrm{i}) k L}+\mathrm{e}^{2 k L}+\mathrm{e}^{(2+2 \mathrm{i}) k L}}
$$

$$
\hat{K}_{\mathrm{b}_{34}}=-\frac{\mathrm{i}\left(-1+\mathrm{e}^{2 \mathrm{i} k L}\right)\left(-1+\mathrm{e}^{2 k L}\right) k^{2}}{1+\mathrm{e}^{2 \mathrm{i} k L}-4 \mathrm{e}^{(1+\mathrm{i}) k L}+\mathrm{e}^{2 k L}+\mathrm{e}^{(2+2 \mathrm{i}) k L}}
$$


(A.10)

$$
\hat{K}_{\mathrm{b}_{44}}=\frac{(1+\mathrm{i})\left(-1+\mathrm{ie}^{2 \mathrm{i} k L}-\mathrm{ie}^{2 k L}+\mathrm{e}^{(2+2 \mathrm{i}) k L}\right) k}{1+\mathrm{e}^{2 \mathrm{i} k L}-4 \mathrm{e}^{(1+\mathrm{i}) k L}+\mathrm{e}^{2 k L}+\mathrm{e}^{(2+2 \mathrm{i}) k L}}
$$

The remaining components of the spectral beam stiffness matrix are found by noting the symmetry of the stiffness matrix.

$$
\hat{\mathbf{K}}_{\mathrm{b}}=\hat{\mathbf{K}}_{\mathrm{b}}^{\mathrm{T}}
$$




\section{APPENDIX B}

\section{Acoustic Excitation \& SPLV Measurement System}

\section{B.1 Acoustic Excitation System}

A schematic of the acoustic excitation system is shown in Figure B.1. The sinusoidal input signal from the PC was first passed through the Adcom audio amplifier (GFA-535) and subsequently through a serially connected first order high pass filter

that consists of three capacitors in parallel. The audio amplifier expected an 8 Ohm load. Thus, a 4 Ohm dummy load was connected in series to the 4 Ohm loudspeaker. The 4 Ohm dummy load is comprised of two 8 Ohm Mills type resistors connected in parallel. Additionally, the audio amplifier has a fixed gain. Hence, the volume for the audio system was adjusted by modifying the gain of the input voltage passing to the audio amplifier via the signal generation program in LabVIEW.

The high pass filter served two purposes. The first was to filter the DC content from the signal coming from the amplifier and passing to the loudspeaker. This assured that the loudspeaker was protected from accidental power spikes. The second purpose was to reduce power transmission through the audio system at frequencies that are damaging to the loudspeaker diaphragm. The DC resistance of the loudspeaker, $R_{1}$, is 2.75 Ohms per the vendor specification [79]. The equivalent DC 


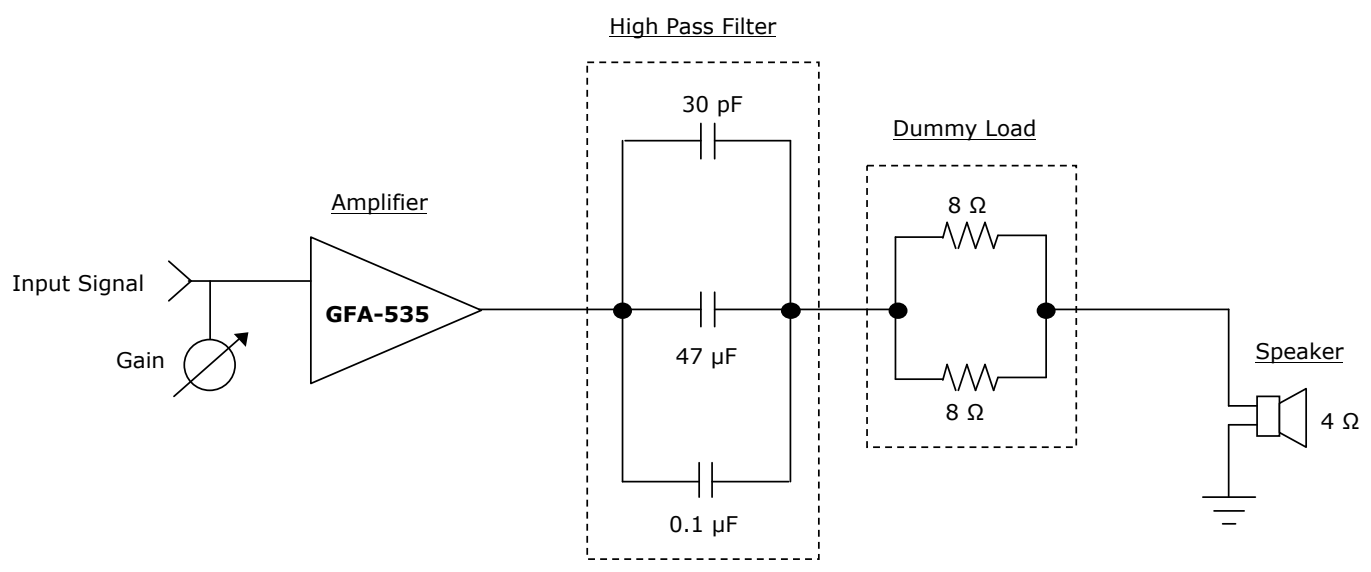

Figure B.1: Schematic of acoustic excitation system

resistance, $R_{\text {eq }}$, of the audio system is then found as

$$
R_{\mathrm{eq}}=R_{\mathrm{d}}+R_{\mathrm{l}}
$$

where $R_{\mathrm{d}}$ is the resistance of the dummy load found through the addition of the Mills type resistors in parallel

$$
R_{\mathrm{d}}=\left(\frac{1}{R_{1}}+\frac{1}{R_{2}}\right)^{-1}
$$

Or, numerically

$$
R_{\mathrm{d}}=\left(\frac{1}{8 \Omega}+\frac{1}{8 \Omega}\right)^{-1}=4 \Omega
$$

From Eqn (B.1), the equivalent DC resistance of the audio system is found to be

$$
R_{\mathrm{eq}}=6.75 \Omega
$$

The resonant frequency of the tweeter is $650 \mathrm{~Hz}$, so the lowest operating frequency of the audio system was selected as $1 \mathrm{kHz}$. A crossover frequency of $500 \mathrm{~Hz}$ was chosen leading to a $3 \mathrm{~dB}$ power loss at this frequency, per Tremaine [99]. This limited power 
sent to the loudspeaker at frequencies near resonance. Given this crossover frequency, the sizing of the first order (i.e. $6 \mathrm{~dB}$ per octave) high pass filter was based on the following equation

$$
f=\frac{1}{2 \pi C_{\mathrm{eq}} R_{\mathrm{eq}}}
$$

where $f$ is the crossover frequency in $\mathrm{Hz}$ and $C_{\text {eq }}$ is the total capacitance of the filter in Farads. Rearranging Eqn (B.5) and substituting numerical values for $f$ and $R_{\text {eq }}$ allows for the calculation of the total capacitance of the filter as

$$
\begin{gathered}
C_{\text {eq }}=\frac{1}{2 \pi R_{\mathrm{eq}} f} \\
C_{\text {eq }}=\frac{1}{2 \pi(6.75 \Omega)(500 \mathrm{~Hz})}=47 \mu \mathrm{F}
\end{gathered}
$$

A non-polarized electrolytic capacitor, $C_{1}=47 \mu \mathrm{F}$, was selected for the crossover design. It was placed in parallel with a mica film capacitor, $C_{2}=30 \mathrm{pF}$, to assist with linearity, and a ceramic bypass capacitor, $C_{3}=0.1 \mu \mathrm{F}$, to carry the alternating current. Observe that the relatively small sizes of the mica film and ceramic bypass capacitors did not effect the magnitude of the capacitance in Eqn (B.7) since capacitors in parallel add like resistors in series as follows

$$
C_{\text {eq }}=C_{1}+C_{2}+C_{3}=47 \mu \mathrm{F}+30 \mathrm{p} F+0.1 \mu \mathrm{F} \approx 47 \mu \mathrm{F}
$$

Accordingly, this filter provided protection against excitation near the resonant frequency of the loudspeaker by limiting power transmission at frequencies below 1 $\mathrm{kHz}$, and it was capable of filtering possibly damaging DC power spikes. The final assembled electrical circuit for the high pass filter, including dummy load, is shown in Figure B.2. 


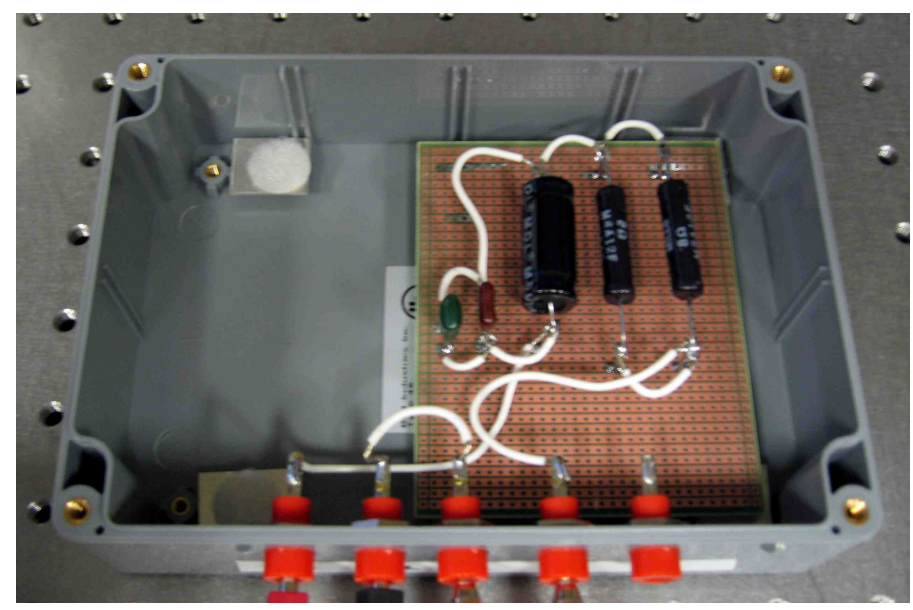

Figure B.2: Assembled high pass filter (including dummy load)

Table B.1: Measurement ranges for velocity decoder OFV-2602, from Polytec [81]

\begin{tabular}{lccc}
\hline Range & $\begin{array}{c}\text { Full Scale Output } \\
\text { (peak to peak) }\end{array}$ & Resolution & $\begin{array}{c}\text { Max. Vibration } \\
\text { Frequency }\end{array}$ \\
\hline $1 \mathrm{~mm} / \mathrm{s} / \mathrm{V}$ & $20 \mathrm{~mm} / \mathrm{s}$ & $0.3 \mu \mathrm{m} / \mathrm{s}$ & $10 \mathrm{kHz}$ \\
$5 \mathrm{~mm} / \mathrm{s} / \mathrm{V}$ & $100(60) \mathrm{mm} / \mathrm{s}$ & $0.3 \mu \mathrm{m} / \mathrm{s}$ & $5(10) \mathrm{kHz}$
\end{tabular}

\section{B.2 SPLV Measurement System}

The output from the SPLV system was connected to the oscilloscope, which provided a sinusoidal voltage signal. The peak amplitude of the signal was manually recorded in units of $\mathrm{mV}$ at each test frequency. Following this, the measured voltage values were converted to velocity values in units of $\mathrm{mm} / \mathrm{s}$ using the appropriate scale factor (i.e. measurement range) setting for the SPLV system. The quality of the velocity measurements from the SPLV system depended upon the distance and alignment between the SPLV sensor head and the test structure. This distance was fixed at $60 \mathrm{~cm}$ and aligned orthogonal to the structure surface element of interest prior to testing per the vendor recommended specifications [81]. The measurement ranges and limitations for the Polytec SPLV model OFV-2602 with OFV-353 optics are provided in Table B.1 for reference. 
BIBLIOGRAPHY 


\section{BIBLIOGRAPHY}

[1] The American Association for the Advancement of Science (AAAS). Noisy days aboard the space station. In Science Magazine's Web site, Retrieved July 25, 2006, from http://www.sciencemag.org/content/vol290/issue5500/rsamples.dtl.

[2] G.K. Ananthasuresh. A new design paradigm for micro-electro-mechanical systems $\mathcal{E}$ investigations on the compliant mechanism synthesis. PhD thesis, University of Michigan: Ann Arbor, 1994.

[3] M. Bettella, M.F. Harrison, and R.S. Sharp. Investigation of automotive creep groan noise with a distributed-source excitation technique. Journal of Sound and Vibration, 255(3):531-547, 2002.

[4] A. Bezazi, F. Scarpa, and C. Remillat. A novel centresymmetric honeycomb composite structure. Composite Structures, 71(3-4):356-364, 2005.

[5] W. V. Bhat. Flight test measurement of exterior turbulent boundary layer pressure fluctuations on Boeing model 737 airplane. Journal of Sound and Vibration, 14(4):439-457, 1971.

[6] W. V. Bhat and J.F. Wilby. Interior noise radiated by an airplane fuselage subjected to turbulent boundary layer excitation and evaluation of noise reduction treatments. Journal of Sound and Vibration, 18(4):449-464, 1971.

[7] M.A. Bhatti. Fundamental finite element analysis and applications: with Mathematica and MATLAB computations. Hoboken: John Wiley \& Sons, 2005.

[8] R.D. Blevins. Formulas for natural frequency and mode shape. R.E. Krieger, 1984.

[9] J.E. Bondaryk. Vibration of truss structures. The Journal of the Acoustical Society of America, 102(4):2167-2175, 1997.

[10] O.M. Bouthier and R.J. Bernhard. Models of space-averaged energetics of plates. American Institute of Aeronautics and Astronautics Journal, 30(3):616623, 1992.

[11] L. Brillouin. Wave propagation in periodic structures: electric filters and crystal lattices. New York: McGraw-Hill, 1946. 
[12] M.K. Bull. Wall-pressure fluctuations beneath turbulent boundary layers: some reflections on forty years of research. Journal of Sound and Vibration, 190(3):299-315, 1996.

[13] C.D. Chapman, K. Saitou, and M.J. Jakiela. Genetic algorithms as an approach to configuration and topology design. Journal of Mechanical Design, 116(4):1005-1012, 1994.

[14] S. Chiras, D. R. Mumm, A. G. Evans, N. Wicks, J. W. Hutchinson, K. Dharmasena, H. N. G. Wadley, and S. Fichter. The structural performance of nearoptimized truss core panels. International Journal of Solids and Structures, 39(15):4093-4115, 2002.

[15] U.S. Department of Defense (DOD). Military handbook - milhdbk-5h: metallic materials and elements for aerospace vehicle structures. In Knovel's Web site, Retrieved August 3, 2006, from http://www.knovel.com/knovel2/Toc.jsp?BookID=754.

[16] H. Douville, P. Masson, and A. Berry. On-resonance transmissibility methodology for quantifying the structure-borne road noise of an automotive suspension assembly. Applied Acoustics, 67(4):358-382, 2006.

[17] J.F. Doyle. Wave propagation in structures: an FFT-based spectral analysis methodology. New York: Springer-Verlag, 1989.

[18] J.F. Doyle and T.N. Farris. A spectrally formulated finite element for flexural wave propagation in beams. The International Journal of Analytical and Experimental Modal Analysis, 5(2):99-107, 1990.

[19] M. El-Raheb. Frequency response of a two-dimensional trusslike periodic panel. The Journal of the Acoustical Society of America, 101(6):3457-3465, 1997.

[20] M. El-Raheb and P. Wagner. Transmission of sound across a trusslike periodic panel; 2-d analysis. The Journal of the Acoustical Society of America, 102(4):2176-2183, 1997.

[21] S.J. Elliott. Active control of structure-borne noise. Journal of Sound and Vibration, 177(5):651-673, 1994.

[22] A.G. Erdman, G.N. Sandor, and S. Kota. Mechanism Design: Analysis and Synthesis, (4th edn.). Upper Saddle River: Prentice Hall, 2001.

[23] D.J. Ewins. Modal testing: theory, practice, and application, (2nd edn.). Philadelphia: Research Studies Press, 2000.

[24] L.L. Faulkner. Handbook of industrial noise control. New York: Industrial Press, 1976.

[25] J. Fish, W. Chen, and G. Nagai. Non-local dispersive model for wave propagation in heterogeneous media: multi-dimensional case. International Journal for Numerical Methods in Engineering, 54(3):347-363, 2002. 
[26] D. Frampton and R. L. Clark. Sound transmission through an aeroelastic plate into a cavity. American Institute of Aeronautics and Astronautics Journal, 35(7):1113-1118, 1997.

[27] M.I. Frecker. Optimal design of compliant mechanisms. PhD thesis, University of Michigan: Ann Arbor, 1997.

[28] C. R. Fuller, S. J. Elliott, and P. A. Nelson. Active control of vibration. San Diego: Academic Press, 1996.

[29] P. Gardonio. Review of active techniques for aerospace vibro-acoustic control. Journal of Aircraft, 39(2):206-214, 2002.

[30] D.E. Goldberg. Genetic algorithms in search, optimization, and machine learning. Reading: Addison-Wesley Pub. Co, 1989.

[31] R.M. Gouker, S.K. Gupta, H.A. Bruck, and T. Holzschuh. Manufacturing of multi-material compliant mechanisms using multi-material molding. International Journal of Advanced Manufacturing Technology, 30(11-12):1049-1075, 2006.

[32] D.S. Grummon, J.A. Shaw, and J. Foltz. Fabrication of cellular shape memory alloy materials by reactive eutectic brazing using niobium. Materials Science and Engineering A-Structural Materials Properties Microstructure and Processing, 438:1113-1118, 2006.

[33] S. Halkjaer, O. Sigmund, and J.S. Jensen. Maximizing band gaps in plate structures. Structural And Multidisciplinary Optimization, 32(4):263-275, 2006.

[34] N.R. Harland, B.R. Mace, and R.W. Jones. Adaptive-passive control of vibration transmission in beams using electro/magnetorheological fluid filled inserts. IEEE Transactions on Control Systems Technology, 9(2):209-220, 2001.

[35] N.R. Harland, B.R. Mace, and R.W. Jones. Wave propagation, reflection and transmission in tunable fluid-filled beams. Journal of Sound and Vibration, 241(5):735-754, 2001.

[36] G.F. Hawkins. Composite materials with embedded machines. U.S. Patent 6,447,871 B1, 1999.

[37] G.F. Hawkins, M.J. O'Brien, R.J. Zaldivar, H.F. Von Bremen, and J.N. Schurr. Development of machine-augmented composites for infrastructure applications. Aerospace Report No. ATR-2004(7796)-1, The Aerospace Corporation, 2003.

[38] J.A. Hetrick and S. Kota. Displacement amplification structure and device. U.S. patent 6,557,436, 2000.

[39] G. W. Housner, L. A. Bergman, T. K. Caughey, A. G. Chassiakos, R. O. Claus, S. F. Masri, R. E. Skelton, T. T. Soong, B. F. Spencer, and J. T. P. Yao. Structural control: Past, present, and future. Journal of Engineering Mechanics, 123(9):897-971, 1997.

[40] L.L. Howell. Compliant mechanisms. New York: Wiley, 2001. 
[41] K.H. Huebner and E.A. Thornton. The finite element method for engineers, (2nd edn.). New York: Wiley, 1982.

[42] T.J.R. Hughes. The finite element method: linear static and dynamic finite element analysis. Mineola: Dover Publications, 2000.

[43] M.I. Hussein, G.M. Hulbert, and R.A. Scott. Dispersive elastodynamics of 1d banded materials and structures: analysis. Journal of Sound and Vibration, 289(4-5):779-806, 2006.

[44] S.R. Idelsohn, E. Onate, F.D. Pin, and N. Calvo. Fluid-structure interaction using the particle finite element method. Computer Methods in Applied Mechanics and Engineering, 195(17-8):2100-2123, 2006.

[45] T. Ishiyama and T. Hashimoto. The impact of sound quality on annoyance caused by road traffic noise: an influence of frequency spectra on annoyance. JSAE Review, 21(2):225-230, 2000.

[46] B.D. Jensen and L.L. Howell. Bistable configurations of compliant mechanisms modeled using four links and translational joints. Journal of Mechanical Design, 126(4):657-666, 2004.

[47] C.D. Johnson. Design of passive damping systems. Journal of Mechanical Design, 117(Sp. Iss. B):171-176, 1995.

[48] C.W. Johnson and I.Y. Mayzus. Cushion connector. U.S. patent 5,224,898, 1993.

[49] M.C. Junger and D. Feit. Sound, structures, and their interaction. Cambridge: MIT Press, 1972.

[50] A.J. Keane. Passive vibration control via unusual geometries: the application of genetic algorithm optimization to structural design. Journal of Sound and Vibration, 185(3):441-453, 1995.

[51] C.J. Kim. A conceptual approach to the computational synthesis of compliant mechanisms. PhD thesis, University of Michigan: Ann Arbor, 2005.

[52] B. Kosko. Noise. New York: Viking, 2006.

[53] S. Kota, S. M. Rodgers, and J. A. Hetrick. Compliant displacement multiplying apparatus for microelectromechanical systems. U.S. patent 6,175,170, 1999.

[54] Y.W. Kwon and H. Bang. The finite element method using MATLAB. Boca Raton: CRC Press, 1997.

[55] R.S. Lakes. Negative poisson ratio polymeric and metallic foams. Journal of Material Science, 23(12):4406-4414, 1988.

[56] R.S. Langley. The response of two-dimensional periodic structures to point harmonic forcing. Journal of Sound and Vibration, 197(4):447-469, 1996. 
[57] U.D. Larsen, O. Sigmund, and S. Bouwstra. Design and fabrication of compliant micromechanisms and structures with negative poisson's ratio. Journal Of Microelectromechanical Systems, 6(2):99-106, 1997.

[58] B.J. Lazan. Damping of materials and members in structural mechanics. New York: Pergamon Press, 1968.

[59] N. Lobontiu. Compliant mechanisms: design of flexure hinges. Boca Raton: CRC Press, 2003.

[60] S. LoBosco and J. LoBosco. Marine propeller assembly with shock absorbing hub and easily replaceable propeller housing. U.S. patent 5,252,028, 1993.

[61] K.J. Lu and S. Kota. An effective method of synthesizing compliant adaptive structures using load path representation. Journal of Intelligent Material Systems and Structures, 16(4):307-317, 2005.

[62] R.H. Lyon. Statistical energy analysis of dynamical systems: theory and applications. Cambridge: MIT Press, 1975.

[63] Military Audiology Association (MAA). Odds and ends. In Noise Protection Health Effects Reduction Web site, Retrieved July 25, 2006, from http://www.militaryaudiology.org/newsletter04/nopher.html.

[64] H. Maddisetty and M. Frecker. Dynamic topology optimization of compliant mechanisms and piezoceramic actuators. Journal of Mechanical Design, 126(6):975-983, 2004.

[65] P.G. Martinsson and A.B. Movchan. Vibrations of lattice structures and phononic band gaps. Quarterly Journal of Mechanics and Applied Mathematics, 56(1):45-64, 2003.

[66] D.M. McCutcheon, J. N. Reddy, M. J. O'Brien, T. S. Creasy, and G. F. Hawkins. Damping composite materials by machine augmentation. Journal of Sound and Vibration, 294(4-5):828-840, 2006.

[67] D.J. Mead and S. Parthan. Free wave propagation in two-dimensional periodic plates. Journal of Sound and Vibration, 64(3):325-348, 1979.

[68] M. Moshrefi-Torbati, A. J. Keane, S. J. Elliott, M. J. Brennan, and E. Rogers. Passive vibration control of a satellite boom structure by geometric optimization using genetic algorithm. Journal of Sound and Vibration, 267(4):879-892, 2003.

[69] T. Murayama. Dynamic mechanical analysis of polymeric material. New York: Elsevier Scientific, 1978.

[70] National Aeronautics \& Space Administration (NASA). Sts112-e-05823. In International Space Station Imagery Web site, Retrieved July 26, 2006, from http://spaceflight.nasa.gov/gallery/images/station/assembly/html/s112e05823. html. 
[71] A.D. Nashif, D.I.G. Jones, and J.P. Henderson. Vibration damping. New York: Wiley, 1985.

[72] The National Institute for Occupational Safety \& Health (NIOSH). Noise and hearing loss prevention. In The National Institute for Occupational Safety and Health's Web site, Retrieved February 8, 2007, from http://www.cdc.gov/niosh/topics/noise/.

[73] A.K. Noor. Contiuum modeling for repetitive lattice structures. Applied Mechanics Review, 41(7):285-296, 1988.

[74] J. Oberg. Orbital shouting - noise becomes a concern on the international space station. Scientific American, 287(1):26-27, 2002.

[75] S.H. Oh, H.S. Kim, and Y.J. Park. Active control of road booming noise in automotive interiors. Journal of the Acoustical Society of America, 111(1):180$188,2002$.

[76] S.J. Olson. Flexible drive shaft coupling. U.S. patent 5,474,499, 1995.

[77] R. Parsons and S.L. Canfield. Developing genetic programming techniques for the design of compliant mechanisms. Structural and Multidisciplinary Optimization, 24(1):78-86, 2002.

[78] J.-F. Paulsen. Resilient couplings. U.S. patent 3,438,221, 1969.

[79] Peerless. Manufacturer's specification - vifa dx25tg05-04. In Parts Express Web site, Retrieved January 7, 2007, from http://www.partsexpress.com/pdf/264-578.pdf.

[80] C. Pierre, M.P. Castanier, and W.J. Chen. Wave localization in multi-coupled periodic structures: application to truss beams. Applied Mechanics Review, 49(2):65-86, 1996.

[81] Polytec. Operator's manual (Man-OFV-2601/2602-9610-e01) - QC vibrometer systems - OFV-2601 and OFV-2602 with OFV-353 optics. Waldbronn, Germany: Polytec GmbH, 1998.

[82] A. Preumont. Vibration control of active structures: an introduction, (2nd edn.). Boston: Kluwer Academic Publishers, 2002.

[83] F. Reuleaux. Model: S39 rhombus straight-line linkage. In Cornell University Kinematic Models for Design Digital Library (KMODDL) Web site, Retrieved August 3, 2006, from http://kmoddl.library.cornell.edu/model.php? $\mathrm{m}=160$.

[84] M. Ruzzene. Vibration and sound radiation of sandwich beams with honeycomb truss core. Journal of Sound and Vibration, 277(4-5):741-763, 2004.

[85] M. Ruzzene, F. Scarpa, and F. Soranna. Wave beaming effects in twodimensional cellular structures. Smart Materials and Structures, 12(3):363$372,2003$. 
[86] L. Saggere. Static shape control of smart structures: a new approach utilizing compliant mechanisms. PhD thesis, University of Michigan: Ann Arbor, 1998.

[87] B.A. Salamon and A. Midha. An introduction to mechanical advantage in compliant mechanisms. Journal of Mechanical Design, 120(2):311-315, 1998.

[88] F. Scarpa, L. G. Ciffo, and J. R. Yates. Dynamic properties of high structural integrity auxetic open cell foam. Smart Materials and Structures, 13(1):49-56, 2004.

[89] M.P. Sepe. Dynamic mechanical analysis for plastics engineering. Morris: Plastics Design Library, 1998.

[90] A.A. Shabana. Vibration of discrete and continuous systems. New York: Springer-Verlag, 1997.

[91] J.A. Shaw and S. Kyriakides. Thermomechanical aspects of niti. Journal of the Mechanics and Physics of Solids, 43(8):1243-1281, 1995.

[92] O. Sigmund and J.S. Jensen. Systematic design of phononic band-gap materials and structures by topology optimization. Philosophical Transactions of the Royal Society A: Mathematical, Physical and Engineering Sciences, 361(1806):1001-1019, 2003.

[93] J. Signorelli and A.H. von Flotow. Wave propagation, power flow, and resonance in a truss beam. Journal of Sound and Vibration, 126-144(1):127-144, 1988.

[94] P.L. Stoffa and M.K. Sen. Nonlinear multiparameter optimization using genetic algorithms - inversion of plane-wave seismograms. Geophysics, 56(11):17941810, 1991.

[95] R.D. Stuart. An introduction to Fourier analysis. New York: Wiley, 1961.

[96] K. Takahashi, K. Usui, E. Abe, and K. Ozaki. Automotoive vibration absorbing joint assembly. U.S. patent 3,848,431, 1974.

[97] C.-Y. Tang, M.J. O'Brien, and G.F. Hawkins. Embedding simple machines to add novel dynamic functions to composites. Journal of Minerals, Metals, and Materials Society, 57(3):32-35, 2005.

[98] T. Tantanawat, Z. Li, and S. Kota. Application of compliant mechanisms to active vibration isolation systems. In Proceedings of the 2004 International Design Engineering Technical Conference (DETC 2004), 2004.

[99] H.M. Tremaine. Audio cyclopedia, (2nd edn.). Indianapolis: H.W. Sams, 1969.

[100] N. Vlahopoulos and X. Zhao. Basic development of hybrid finite element method for midfrequency structural vibrations. American Institute of Aeronautics and Astronautics Journal, 37(11):1495-1505, 1999.

[101] H. Wadley. Multifunctional periodic cellular metals. Philosophical Transactions of the Royal Society A: Mathematical, Physical and Engineering Sciences, 364(1838):31-68, 2006. 
[102] J. C. Wallach and L. J. Gibson. Mechanical behavior of a three-dimensional truss material. International Journal of Solids and Structures, 38(40-41):71817196, 2001.

[103] V.W. Wang. A unit cell approach for lightweight structure and compliant mechanism. PhD thesis, Georgia Institute of Technology, 2005.

[104] G. Winter. Genetic algorithms in engineering and computer science. New York: John Wiley and Sons, 1995.

[105] M. Yalcintas and H.M. Dai. Vibration suppression capabilities of magnetorheological materials based adaptive structures. Smart Materials \& Structures, 13(1):1-11, 2004.

[106] T.Y. Yang. Finite element structural analysis. Englewood Cliffs: PrenticeHall, 1986.

[107] Ç. Yilmaz. Analysis and design of uniaxial passive vibration isolators considering stiffness and bandwidth limitations. PhD thesis, University of Michigan: Ann Arbor, 2005.

[108] Ç. Yilmaz and N. Kikuchi. Analysis and design of passive low-pass filter-type vibration isolators considering stiffness and mass limitations. Journal of Sound and Vibration, 293(1-2):171-195, 2006.

[109] Y.Q. Yu, L.L. Howell, C. Lusk, Y. Yue, and M.G. He. Dynamic modeling of compliant mechanisms based on the pseudo-rigid-body model. Journal of Mechanical Design, 127(4):760-765, 2005. 


\begin{abstract}
ANALYSIS, DESIGN, AND OPTIMIZATION OF STRUCTURES WITH INTEGRAL COMPLIANT MECHANISMS FOR MID-FREQUENCY RESPONSE

by

Ercan M. Dede
\end{abstract}

Chair: Gregory M. Hulbert

The vibration of lightweight structures in the $1 \mathrm{kHz}$ to $10 \mathrm{kHz}$ middle frequency region generates noise, which has adverse effects on human performance and perception of quality. Typical solutions, such as spring-mass absorbers, visco-elastic coatings, and active control, are effective across these frequencies. Nonetheless, they often lead to greater system complexity or weight. Accordingly, the objective of this research is to introduce a new technique for the reduction of middle frequency structural-borne noise.

In reducing mid-frequency response, a multi-scale technique based on amplification principles is explored to integrate small-scale compliant mechanisms into largescale structures. Specifically, the principle of mechanical advantage is examined as a mechanism design tool to reduce energy transmission. An efficient spectral finite element computational approach is exploited for basic force-velocity and energy flow analyses of both two-dimensional and three-dimensional structures. A genetic algorithm is employed to optimize structure topology and size for greatest effectiveness 
in the frequency range of interest. The results of prototype testing using acoustic excitation and laser interferometry measurement techniques are presented to validate computational predictions of structural dynamic response. These investigations indicate that a significant decrease in structural vibration is achievable, and they suggest promising applications including the design of multi-functional structuralacoustic panels for broadband vehicle noise reduction.

In summary, there are three primary contributions of this research. First, the computational methods required for the analysis and design of structures with integral compliant mechanisms are synthesized. Second, a novel methodology is established as an integral part of the structural design process for the reduction of mid-frequency structural-borne noise. Third, the feasibility of this method is experimentally validated. Hence, the field of dynamic analysis is extended towards solving practical problems through the synthesis of several existing research fields including structural dynamics, compliant mechanism design, finite element computational analysis, and optimization via evolutionary algorithms. 